\title{
Design Software Methods for Strengthening of Reinforced Concrete Beams and Columns
}

Trevor D. Saas

West Virginia University

Follow this and additional works at: https://researchrepository.wvu.edu/etd

\section{Recommended Citation}

Saas, Trevor D., "Design Software Methods for Strengthening of Reinforced Concrete Beams and Columns" (2013). Graduate Theses, Dissertations, and Problem Reports. 4998.

https://researchrepository.wvu.edu/etd/4998

This Thesis is protected by copyright and/or related rights. It has been brought to you by the The Research Repository @ WVU with permission from the rights-holder(s). You are free to use this Thesis in any way that is permitted by the copyright and related rights legislation that applies to your use. For other uses you must obtain permission from the rights-holder(s) directly, unless additional rights are indicated by a Creative Commons license in the record and/ or on the work itself. This Thesis has been accepted for inclusion in WVU Graduate Theses, Dissertations, and Problem Reports collection by an authorized administrator of The Research Repository @ WVU. For more information, please contact researchrepository@mail.wvu.edu. 


\title{
Design Software Methods for Strengthening of Reinforced Concrete Beams and Columns
}

\author{
Trevor D. Saas
}

Thesis submitted to the Benjamin M. Statler College of Engineering and Mineral Resources at West Virginia University in partial fulfillment of the requirements for the degree of

Master of Science

in

Civil Engineering

Ever J. Barbero, Ph.D., Chair Eduardo Sosa, Ph.D.

Udaya Halabe, Ph.D.

Department of Civil and Environmental Engineering

Morgantown, West Virginia

2013

Keywords: fiber reinforced polymer (FRP); composites; FRP strengthening design; reinforced concrete design; objected-oriented programming; 


\section{ABSTRACT \\ Design Software Methods for Strengthening of Reinforced Concrete Beams and Columns}

Trevor D. Saas

Using fiber reinforced polymers (FRP) to strengthen dilapidated and failing reinforced concrete structures is becoming more frequent and often a cheaper alternative to traditional strengthening methods. Computer Aided Design Environment for Composites (CADEC) assists in the design and analysis of composite materials but lacks a user-friendly environment for the design and verification of strengthened reinforced concrete beams and columns. Therefore, design software methods are needed to develop an add-on application.

The development of the design software methods was done using $\mathrm{C \#}$ (pronounced " $\mathrm{C}$ Sharp"), an object-orientated language, because of the object classes. The object classes were used to allow other design philosophies to be implemented when creating usable applications. The object classes are organized into required strengthening (bending, shear, pure axial, and combination of axial and bending) and major design steps (preliminary design, original design, FRP configuration and design, and verification). An application was created to validate the design software methods. The application was created using Windows Forms that were organized into four paths (beams in flexure, beams in shear, columns, and beam-columns). Each path allowed the users to enter required parameters and then used associated object classes to automate the design process with minimal user involvement.

Analysis of the developed application using available strengthening design examples resulted in identical or very minor differences between the object classes used and the examples. Any major differences were anticipated because of the design of the class methods requiring a percent error. Overall, the object classes were effective; though some of the methods were often redundant and inefficient. The application was also over simplified allowing potential user errors to happen if the user is not familiar with the application's operation. 


\section{Acknowledgments}

I would like to thank Dr. Ever Barbero, my research advisor, for giving me the opportunity to work on such a challenging and rewarding project. His knowledge and guidance of composites materials allowed me to explore and digest a whole new area of expertise for me and gave me new skill sets in object-oriented programming.

I would also like to thank John Wattick for introducing me to Dr. Barbero. For without that connection I would not have been at WVU. As well, I'd like to thank John for his continuing support and friendship through my years at WVU.

I want to thank Dr. Robin Hensel for giving me an opportunity to work for WVU Freshman Engineering Department and for allowing me to teach Engineering 101. The experience with Freshman Engineering was extremely rewarding and allowed me to develop not only as an engineer with better communication, organization and leadership skills, but also as an individual. Teaching incoming freshmen about the design process and technical communications will be missed.

Lastly, I'd like to thank my family for their support and distilling me with passion, work ethic, and my sense of individuality. They have always pushed me to follow my dreams and passions. 


\section{Table of Contents}

ABSTRACT

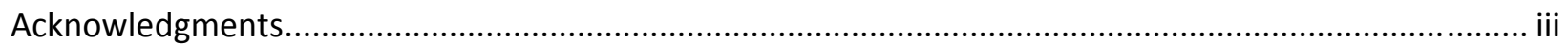

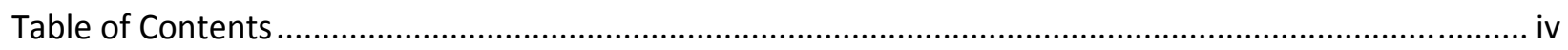

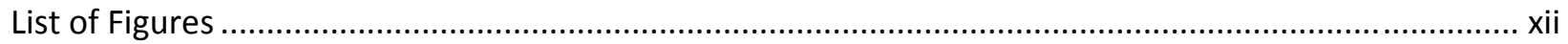

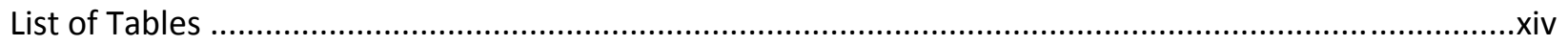

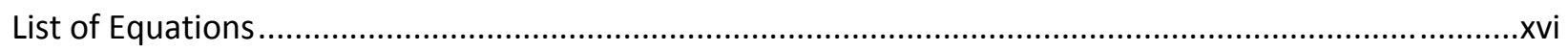

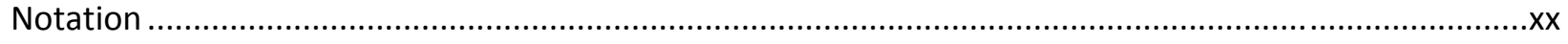

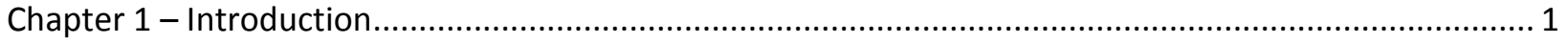

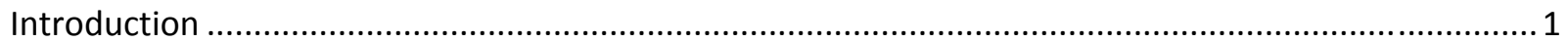

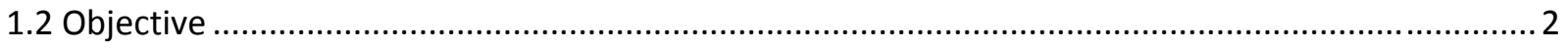

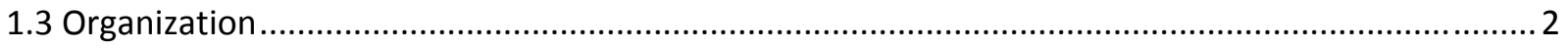

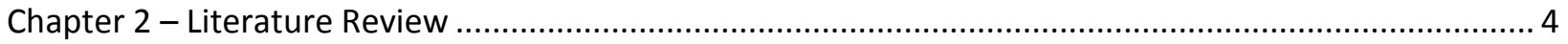

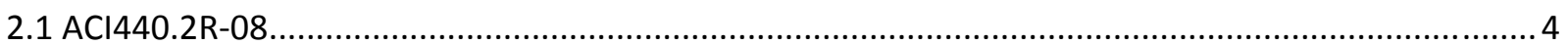

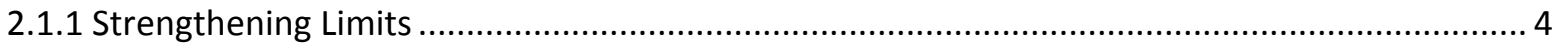

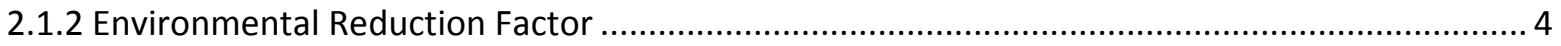

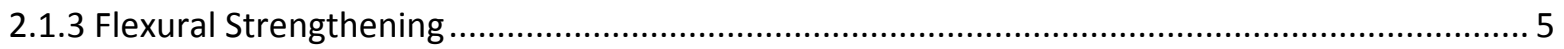

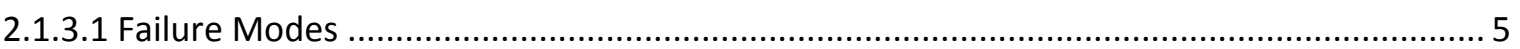

2.1.3.2 Reinforced Concrete Member Assumptions......................................................................... 6

2.1.3.3 Stress and Stain Levels in FRP Reinforcement ............................................................ 6

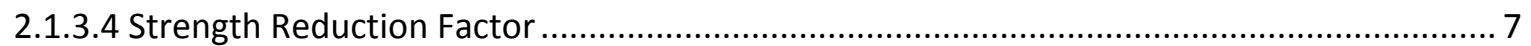

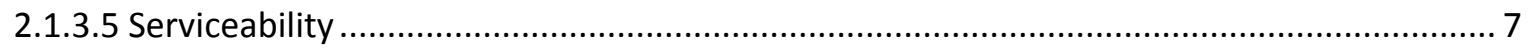

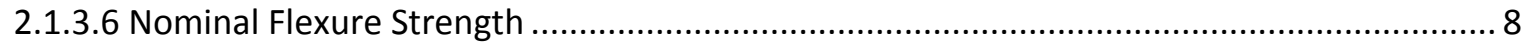

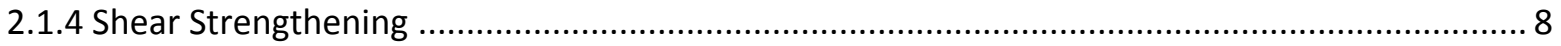

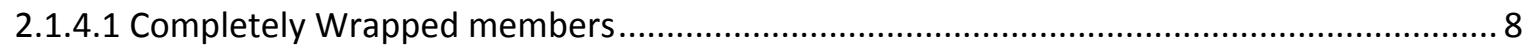

2.1.4.2 Bonded U-wraps or Bonded Face Plies ....................................................................... 9

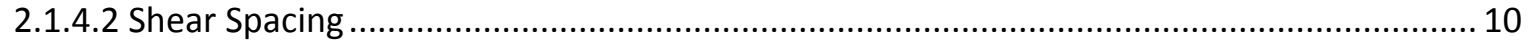

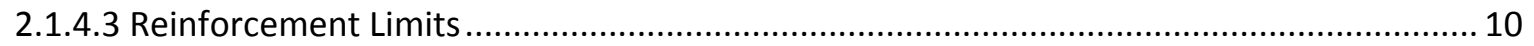

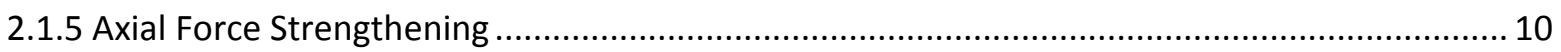

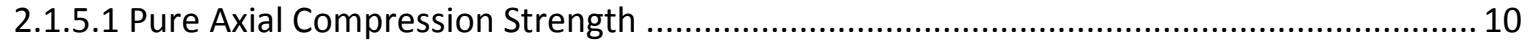

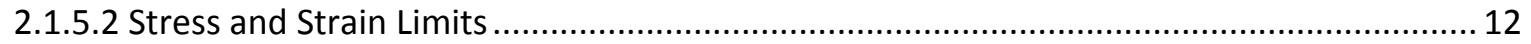




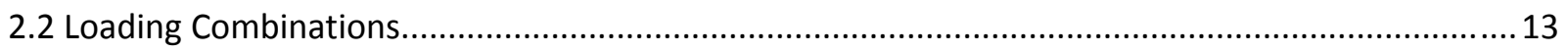

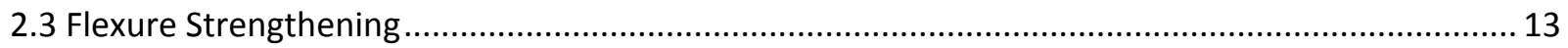

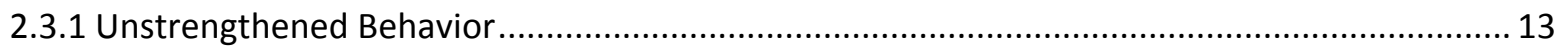

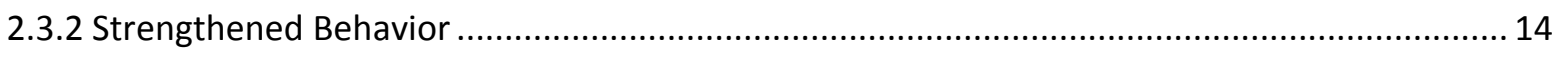

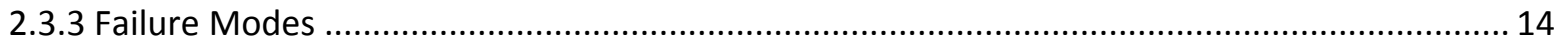

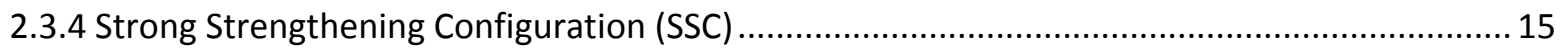

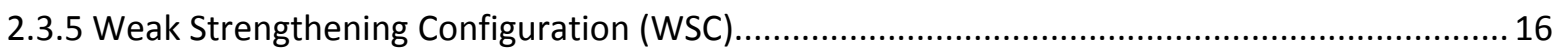

2.3.6 Balanced Strengthening Configuration (BSC) .................................................................. 16

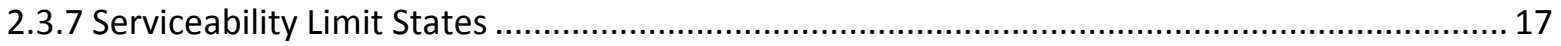

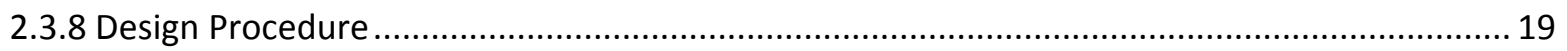

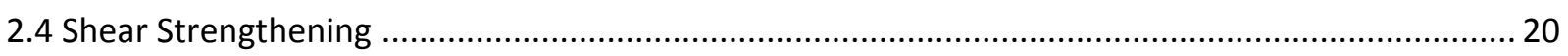

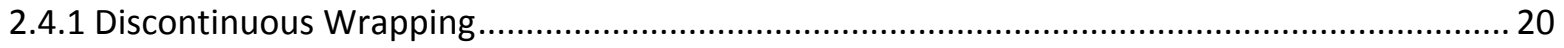

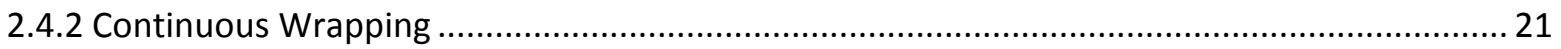

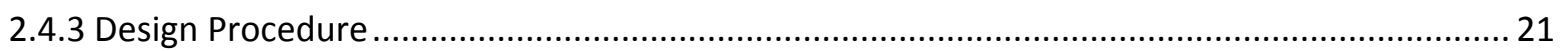

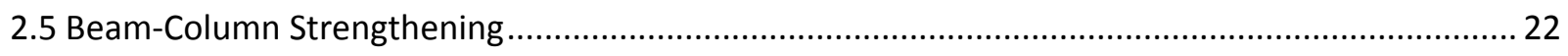

2.5.1 Pure Axial Compression Design Procedure .......................................................................... 22

2.5.2 Beam-Column Verification Procedure …............................................................................ 23

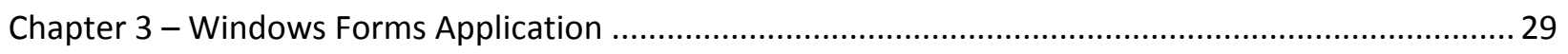

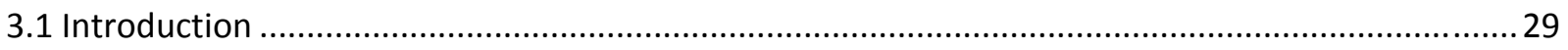

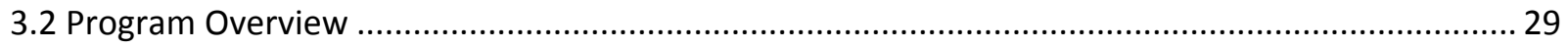

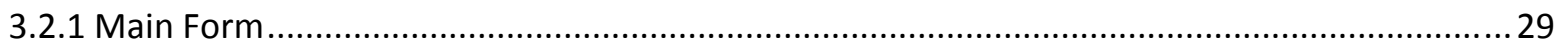

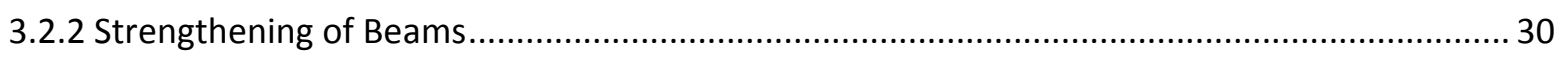

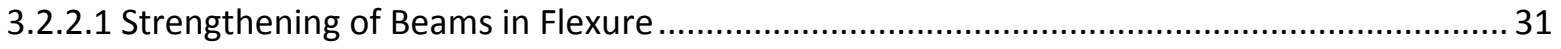

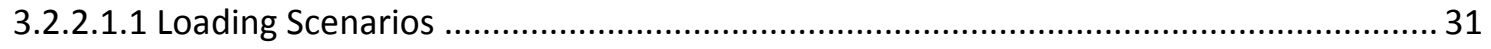

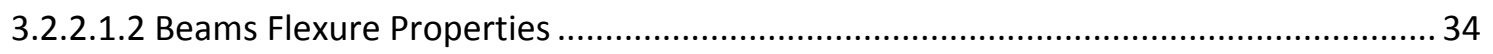

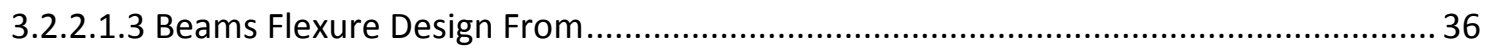

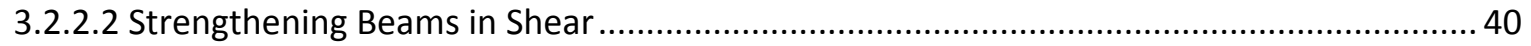

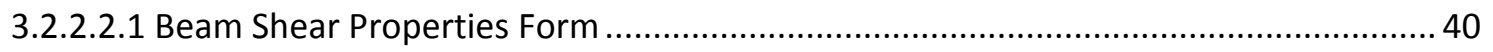

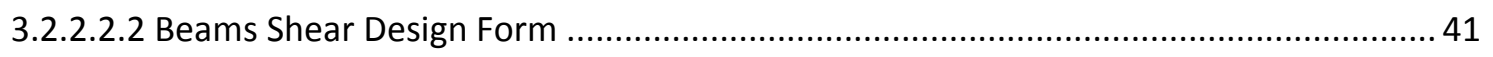

3.2.3 Strengthening of Column in Pure Axial Compression ............................................................ 44

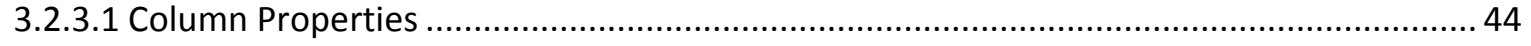

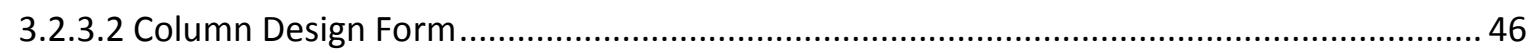




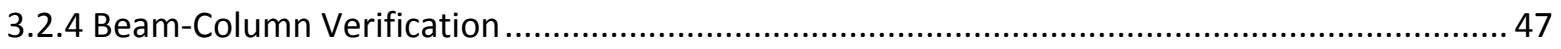

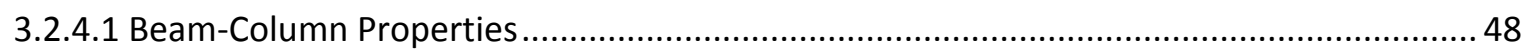

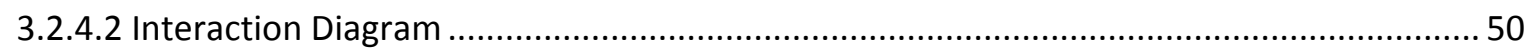

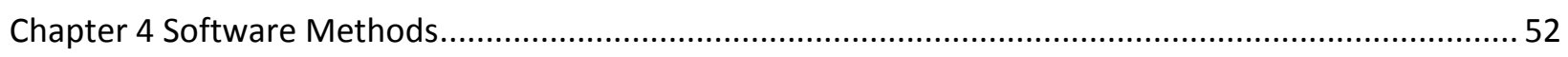

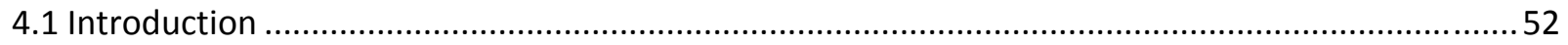

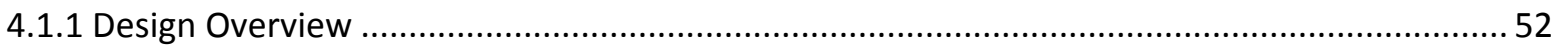

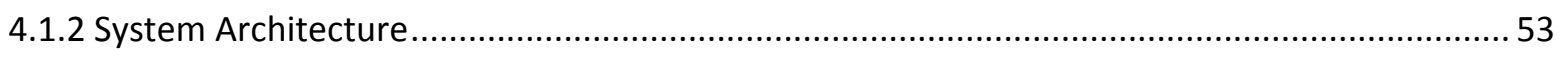

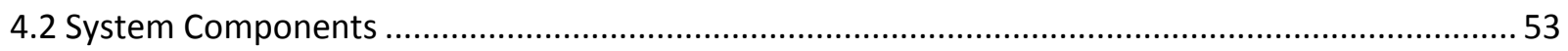

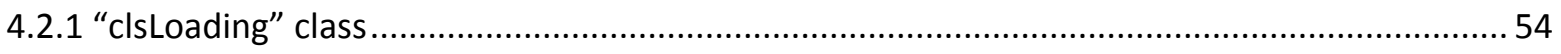

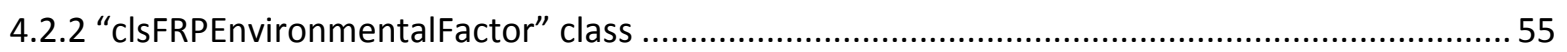

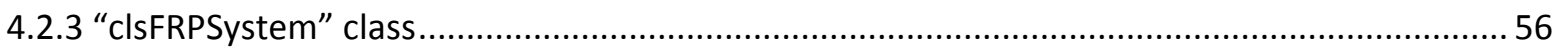

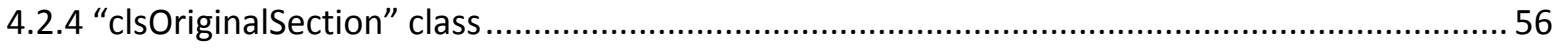

4.2.4.1 "MinimumLoadCapacityOfUnstrengthendColumn" method ............................................57

4.2.4.2 "UnstrengthenedCapacityOfUnstrengthenedColumn" method ......................................57

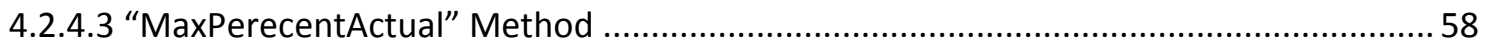

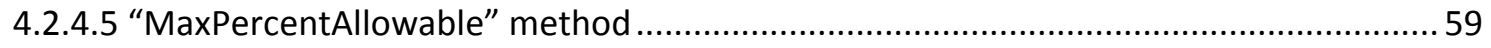

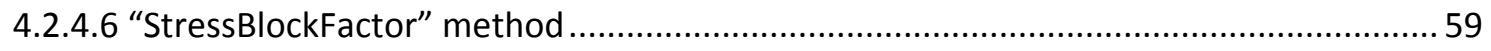

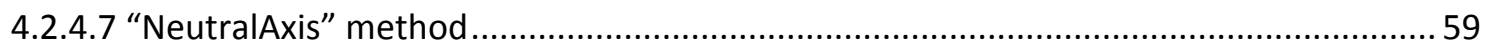

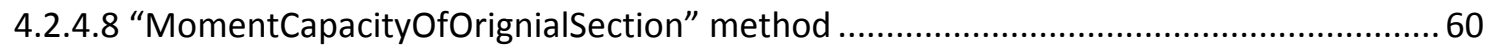

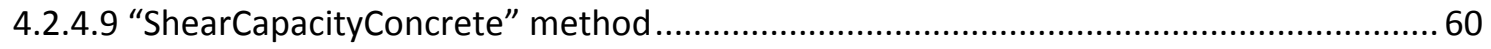

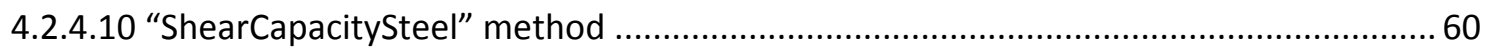

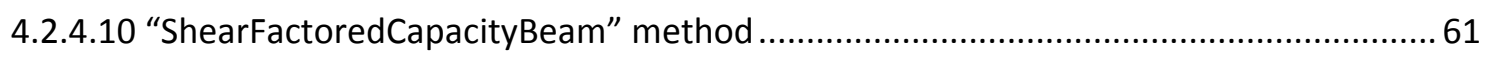

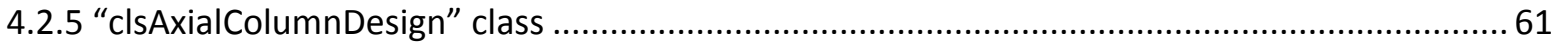

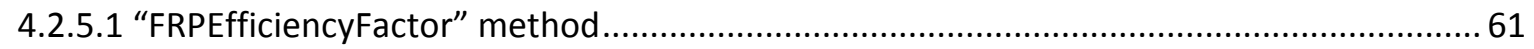

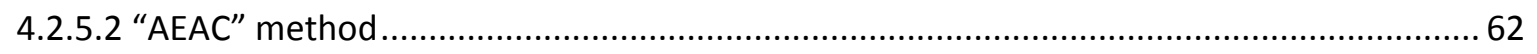

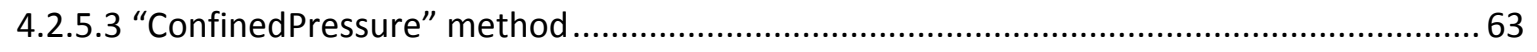

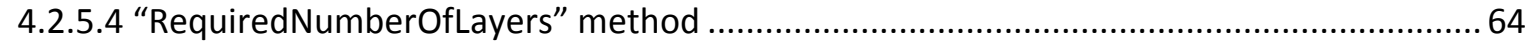

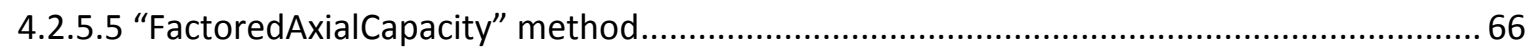

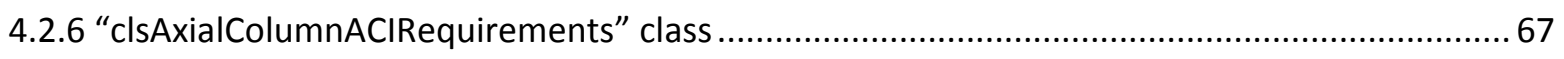

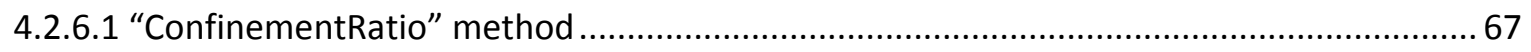

4.2.6.2 "CompressiveStrainOnConfinedColumn" method ........................................................... 68

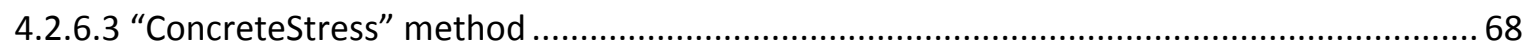




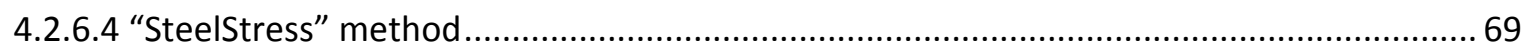

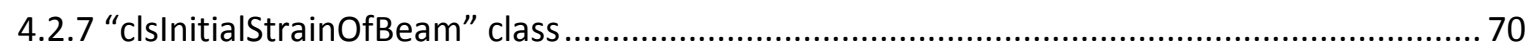

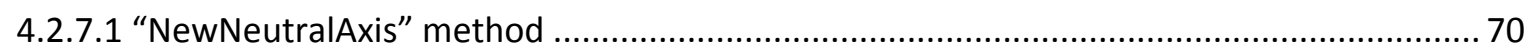

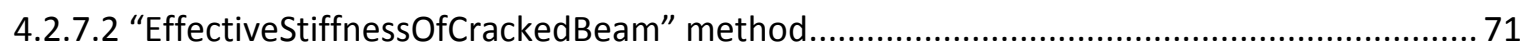

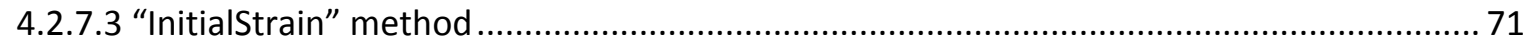

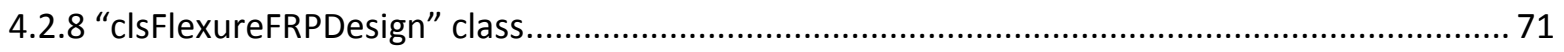

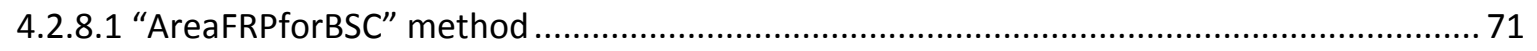

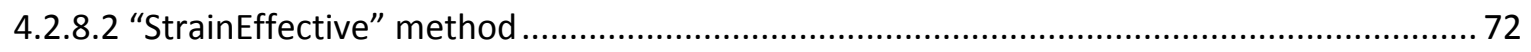

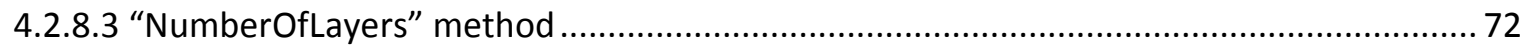

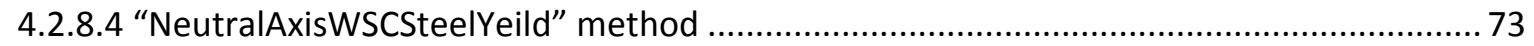

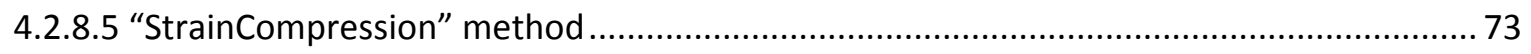

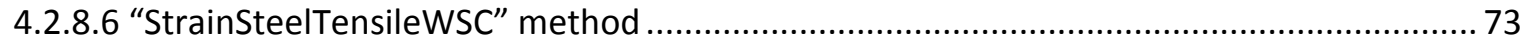

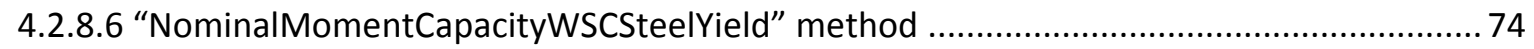

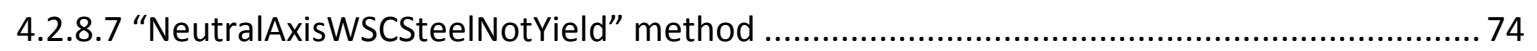

4.2.8.8 “NominalMomentCapacityWSCSteelNotYield" method ................................................. 75

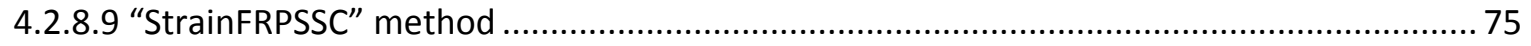

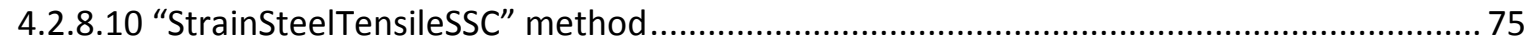

4.2.8.11 "NeutralAxisSSCSteelYielding" method ....................................................................... 76

4.2.8.12 "NominalMomentCapacitySSCSteelYield" method ......................................................... 76

4.2.8.13 “NeutralAxisSSCSteelNotYield" method ........................................................................ 77

4.2.8.14 "NominalMomentCapacitySSCSteelNotYield" method ................................................... 77

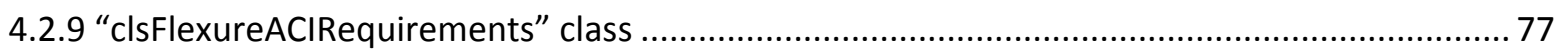

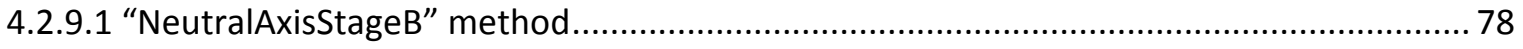

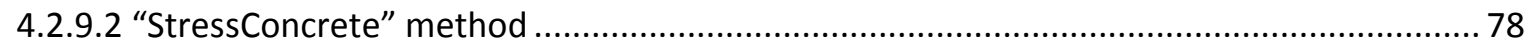

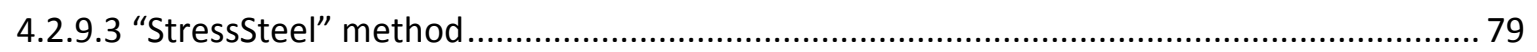

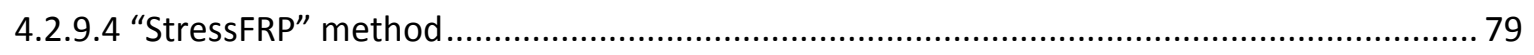

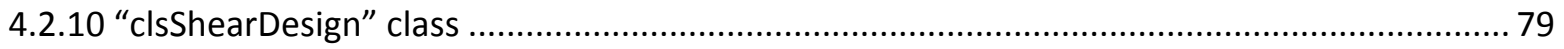

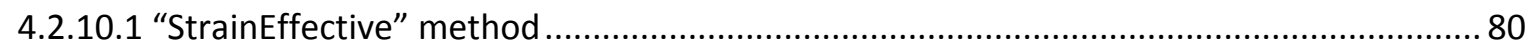

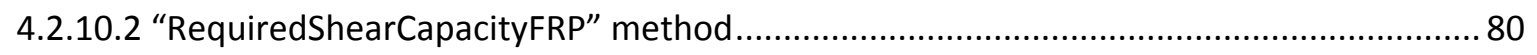

4.2.10.3 “NumberOfLayersContinouslyWrapped” method ........................................................... 81

4.2.10.4 "ShearCapacityFRPContinousWrapping" method ........................................................... 81

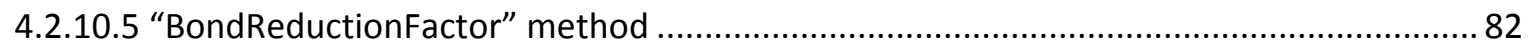




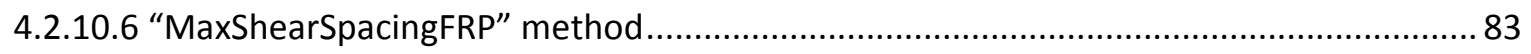

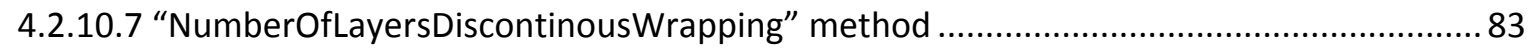

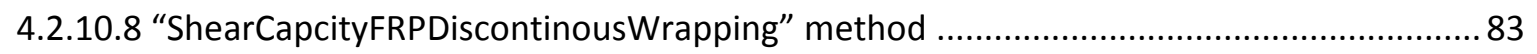

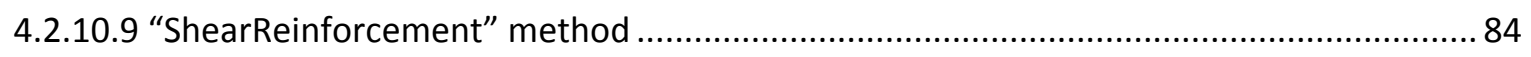

4.2.10.10 "MaxAllowableShearReinforcement" method ............................................................ 84

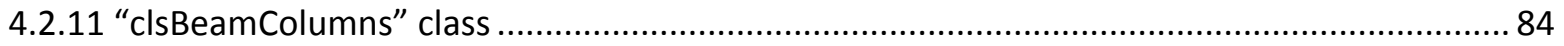

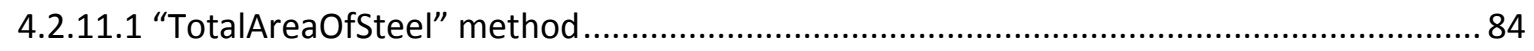

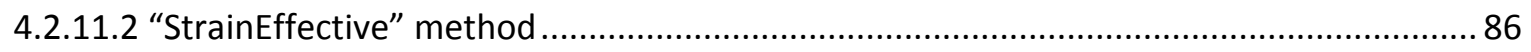

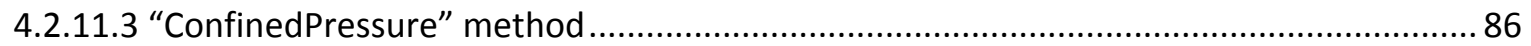

4.2.11.4 "UltimateAxialCompressionStrainOfConfinedConcrete" method ................................... 86

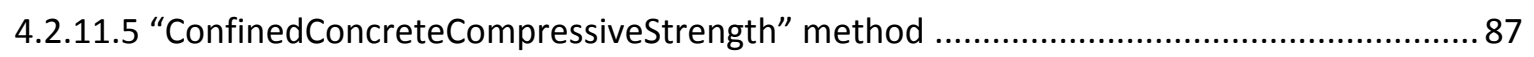

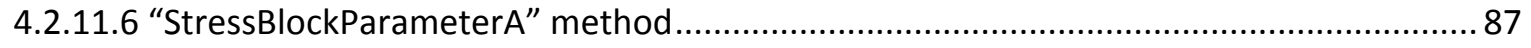

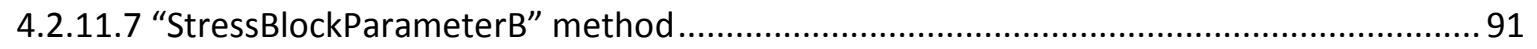

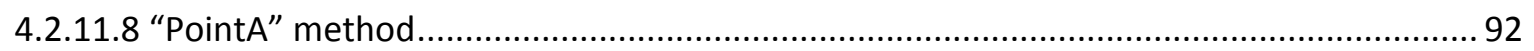

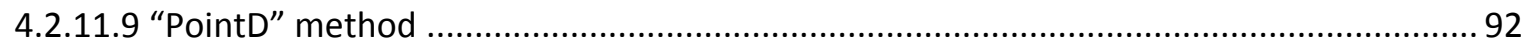

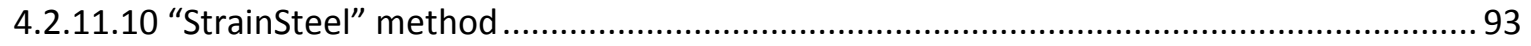

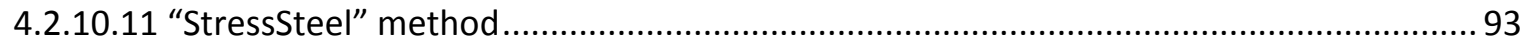

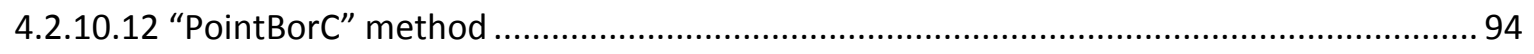

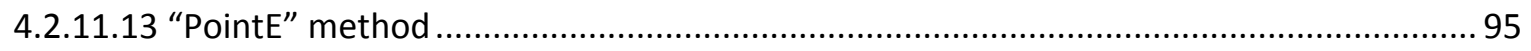

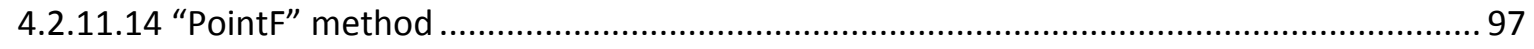

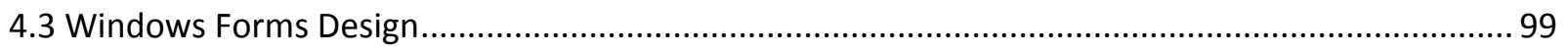

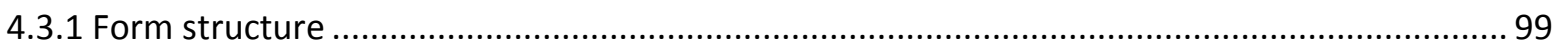

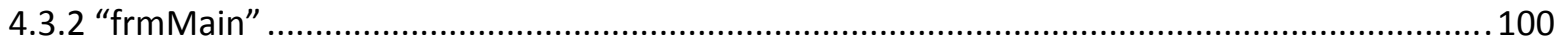

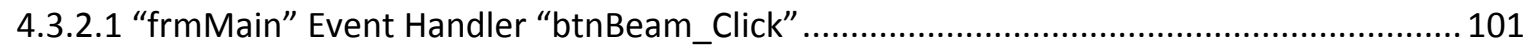

4.3.2.2 "frmMain" Event Handler "btnColumn_Click" .............................................................. 101

4.3.2.3 "frmMain" Event Handler "btnBeamColumn_Click" .................................................... 101

4.3.2.4 "frmMain” Event Handler "btnClose_Click" ....................................................................... 101

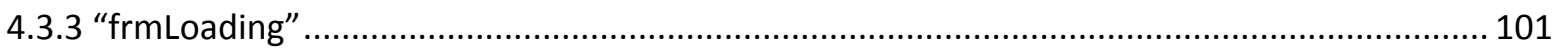

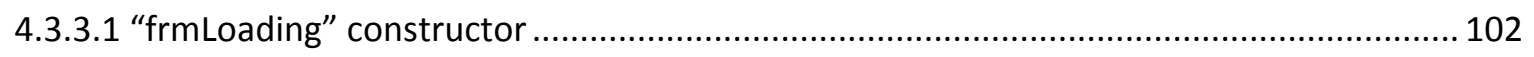

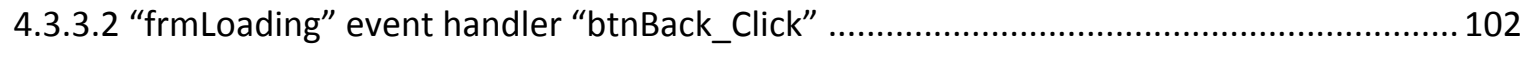

4.3.3.3 "frmLoading" event handler "btnContinue_Click" ........................................................ 102

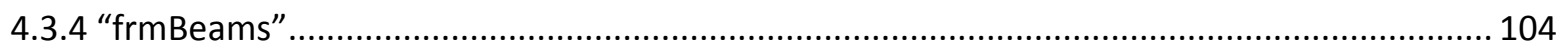


4.3.5.1 "frmFlexureBeamProperties" event handler "btnContinue_Click" 105

4.3.6 "frmFlexureResults". 106

4.3.6.1 "frmFlexureResults" event handler "btnCalculateResults_Click" ................................... 107

4.3.6.2 "frmFlexureResults" event handler "btnContinue_Click" .............................................. 109

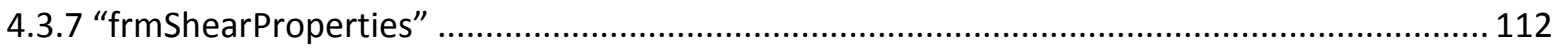

4.3.7.1 "frmShearProperties" event handler "btnContinue_Click" .......................................... 113

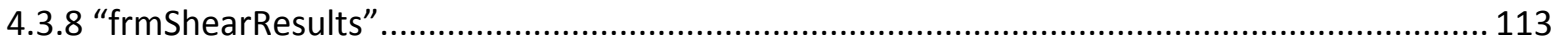

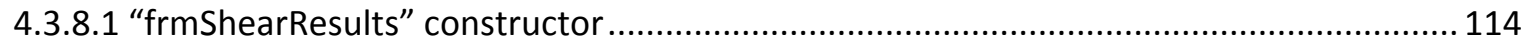

4.3.8.2 "frmShearResults" event handler "rbtComplete_CheckedChanged" ..............................114

4.3.8.3 "frmShearResults" event handler "rbt3Sided_CheckedChanged" ................................. 114

4.3.8.4 "frmShearResults" event handler "rbt2Sided_CheckedChanged" ................................... 115

4.3.8.5 "frmShearResults" event handler "rbtContinous_CheckedChanged"............................. 115

4.3.8.6 "frmShearResults" event handler "rbtDiscontinous_CheckedChanged" ......................... 115

4.3.8.7 "frmShearResults" event handler "btnCalculateResults_Clicked" ................................. 116

4.3.8.8 "frmShearResults" event handler "txtUserInputNumberOfLayers_TextChanged" ...........118

4.3.8.9 "frmShearResults" event handler "btnContinue_Click" ................................................. 119

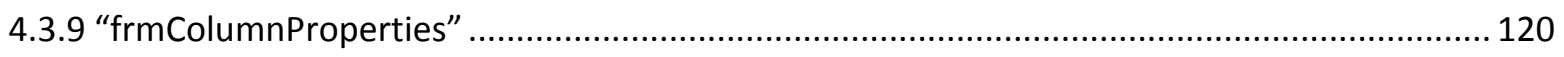

4.3.9.1 "frmColumnProperties" event handler "rbtnCircular_CheckedChanged" ......................... 121

4.3.9.2 "frmColumnProperties" event handler "rbtnRectangular_CheckedChanged" ................. 121

4.3.9.3 "frmColunProperties" event handler "txtDiameter_LostFocus" ..................................... 121

4.3.9.4 "frmColumnProperties" event handler "txtBaseOfColumn_LostFocus" ........................... 122

4.3.9.5 "frmColumnProperties" event handler "txtHeightOfColumn_LostFocus" ........................ 122

4.3.9.6 "frmColumnProperties" event handler "btnContinue_Clicked" ...................................... 123

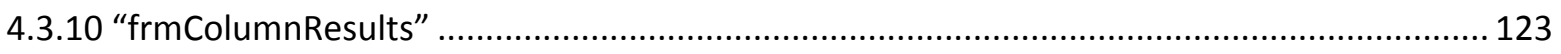

4.3.10.1 "frmColumnResults" event handler "btnCalculateResults" ......................................... 123

4.3.10.2 "frmColumnResults" event handler "btnContinue_Clicked" ........................................ 124

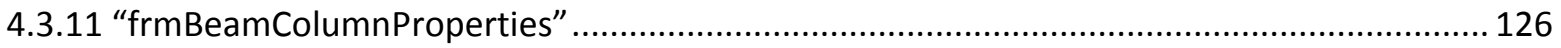

4.3.11.1 "frmBeamColumnProperties" event handler "txtDistanceOfReinforcement_LostFocus"

4.3.11.2 "frmBeamColumnProperties" event handler "txtAreaOfSteel_LostFocus" ...................128

4.3.11.3 "frmBeamColumnProperties" event handler "btnContinue_Clicked" ........................... 128 


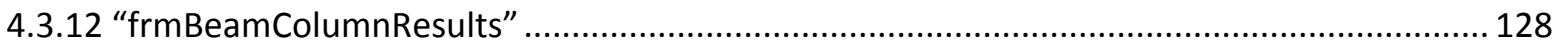

4.3.12.1 "frmBeamColumnResults" event handler "btnShowResults_Click" .............................. 129

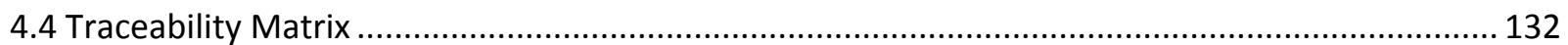

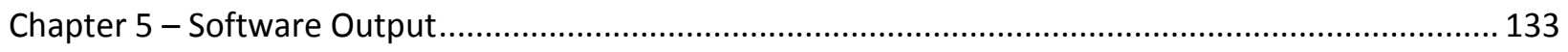

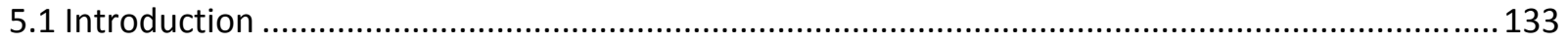

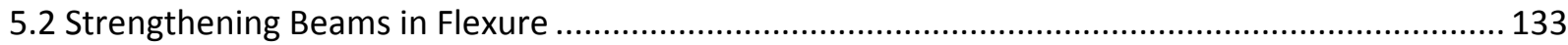

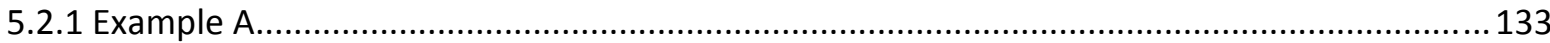

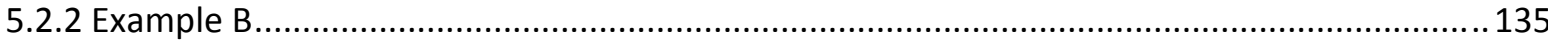

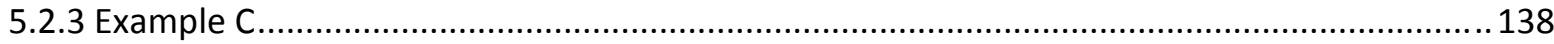

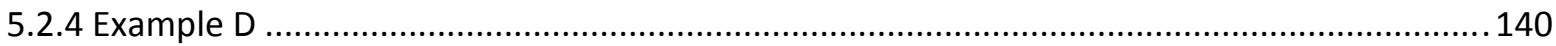

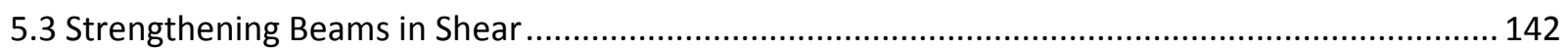

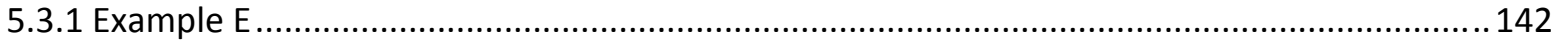

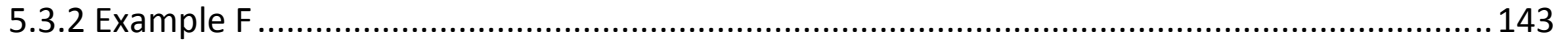

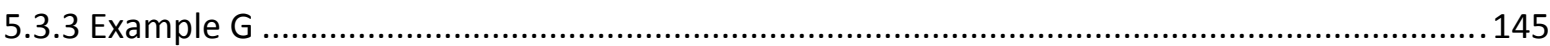

5.4 Strengthening Columns in Pure Axial Compression............................................................. 147

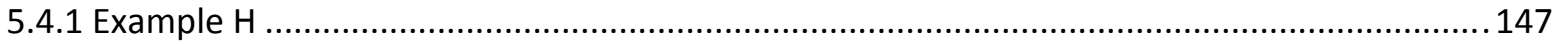

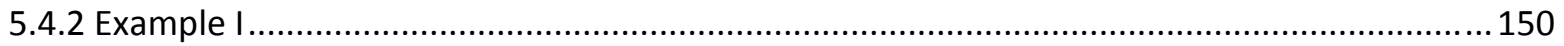

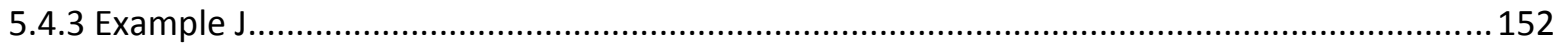

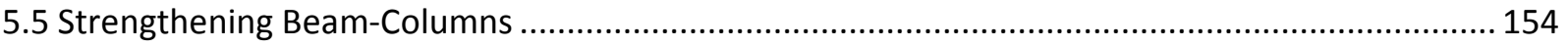

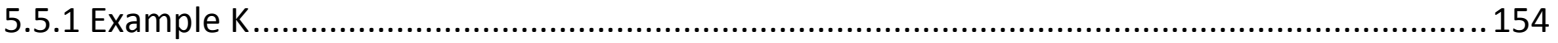

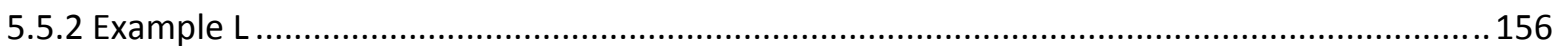

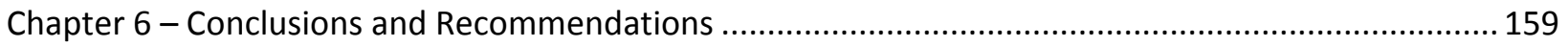

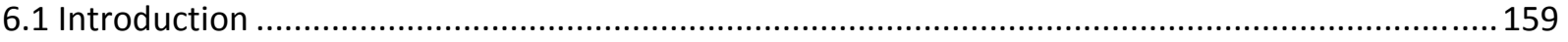

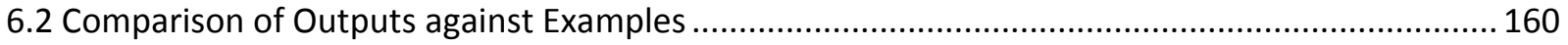

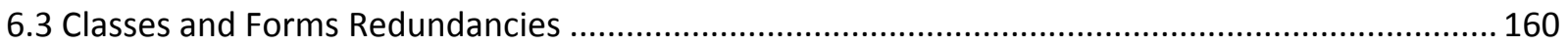

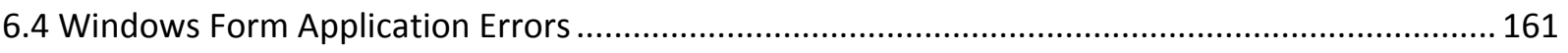

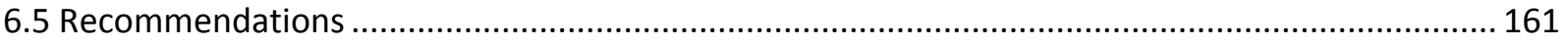

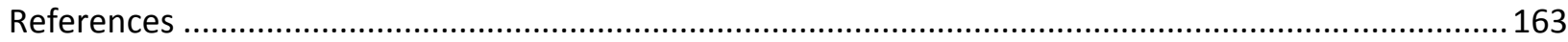

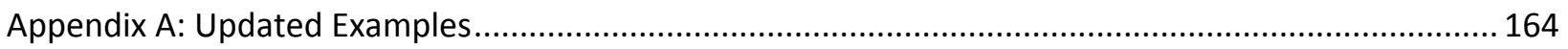

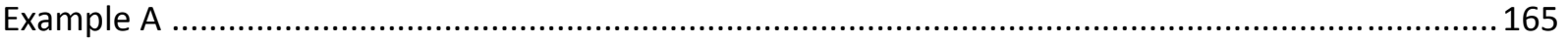

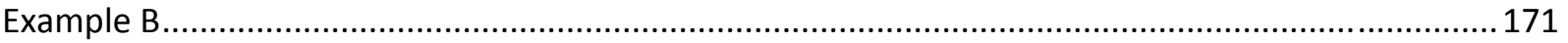

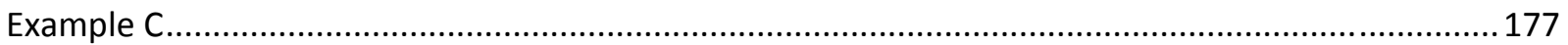




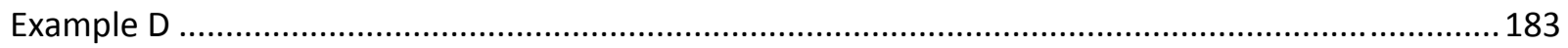

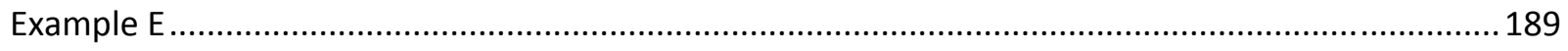

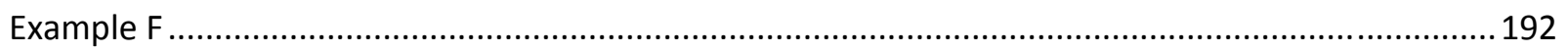

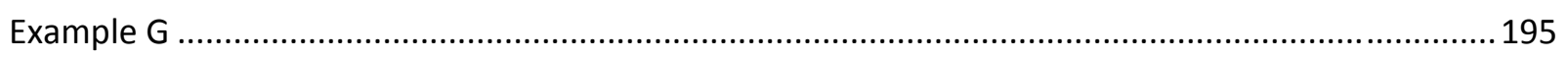

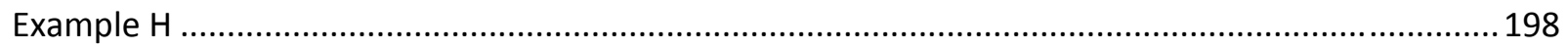

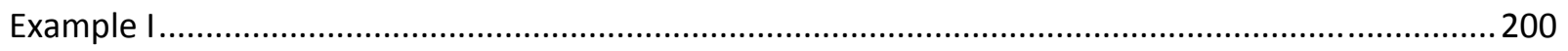

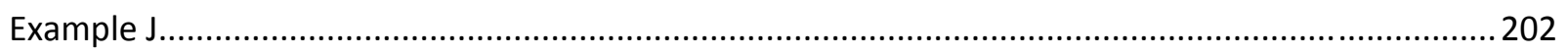

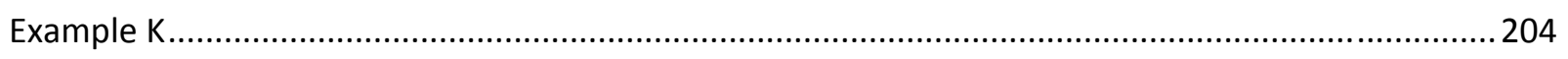

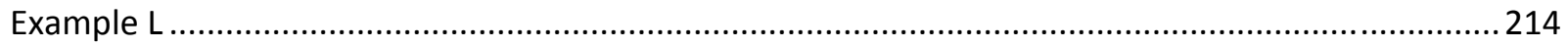




\section{List of Figures}

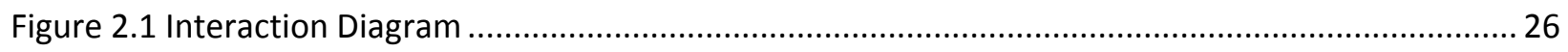

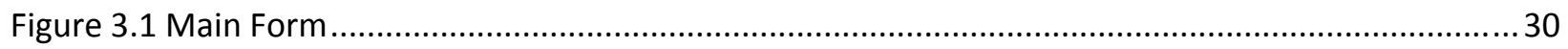

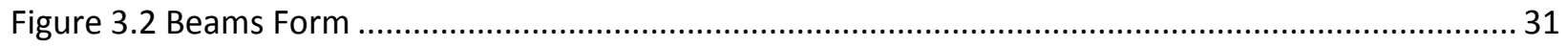

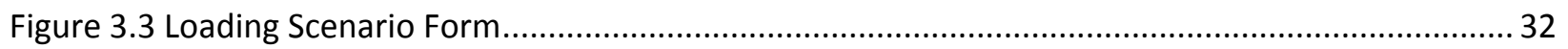

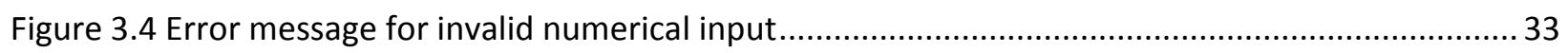

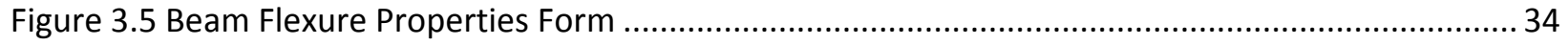

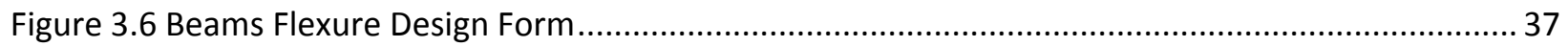

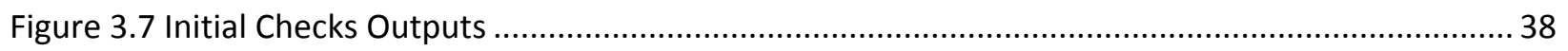

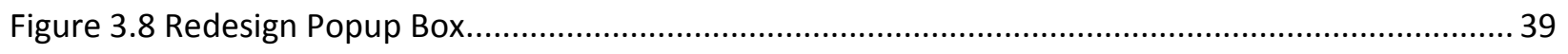

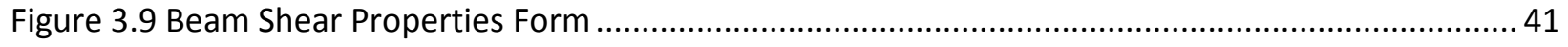

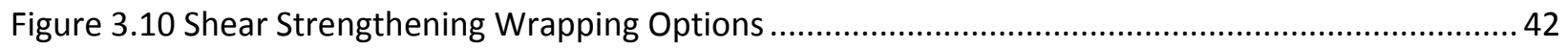

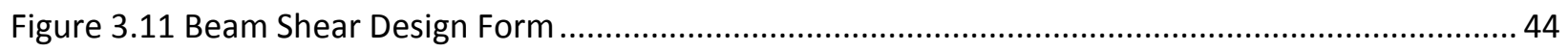

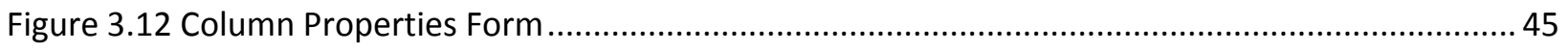

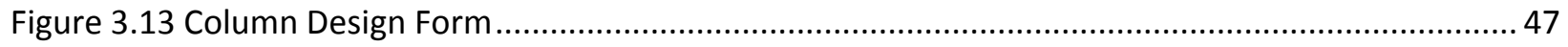

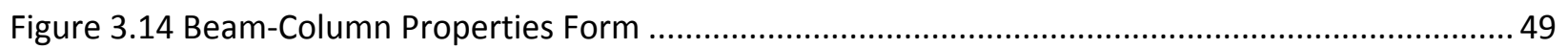

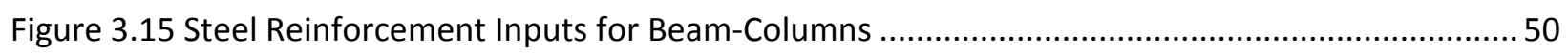

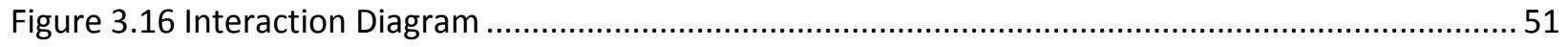

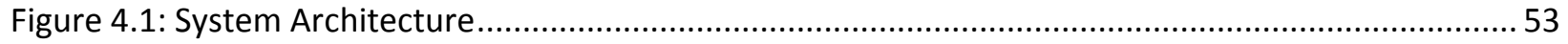

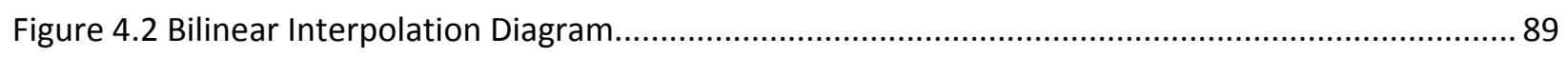

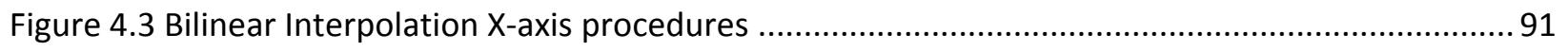

Figure 4.4 General Structure for checking for valid user inputs ......................................................... 103

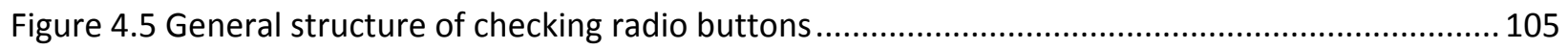

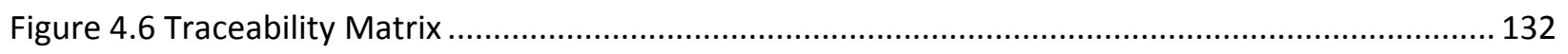

Figure 5.1 Example A: Windows Application Inputs ...................................................................... 134

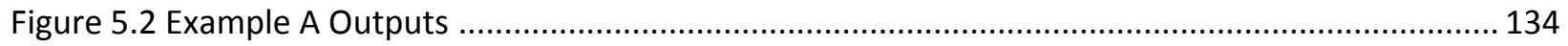

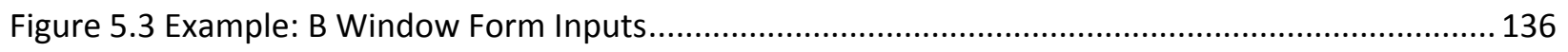

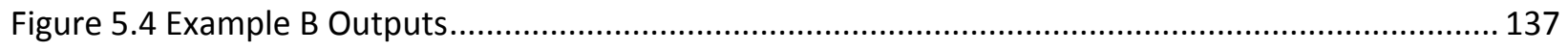

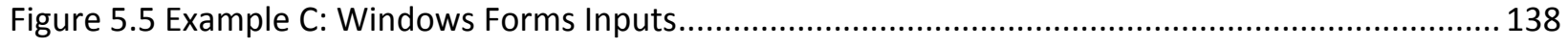




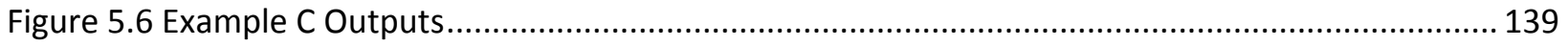

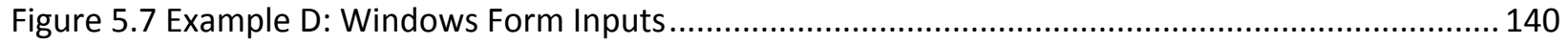

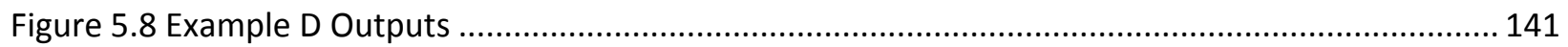

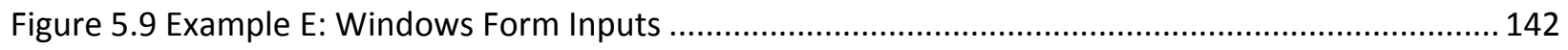

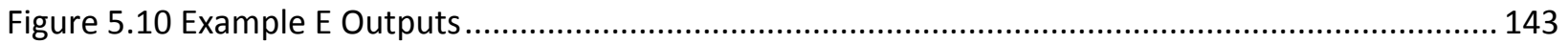

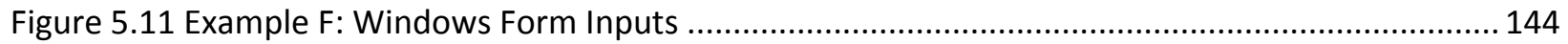

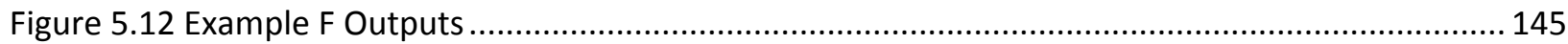

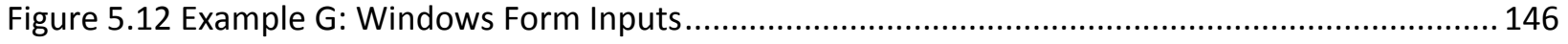

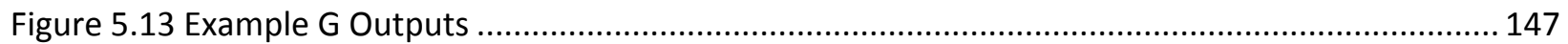

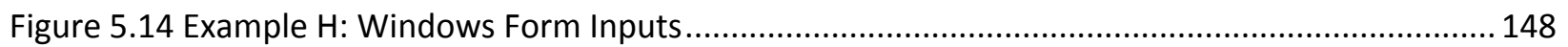

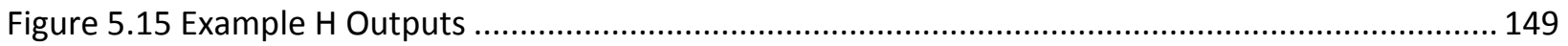

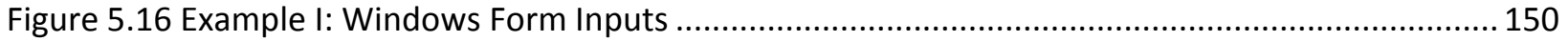

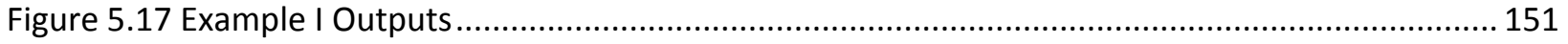

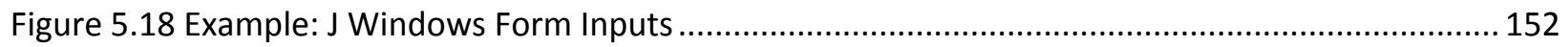

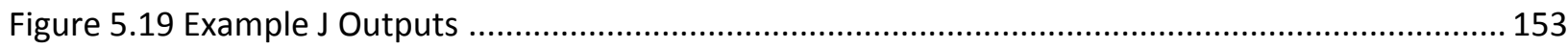

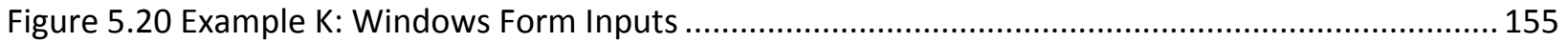

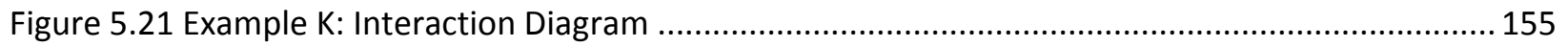

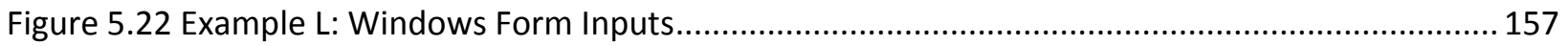

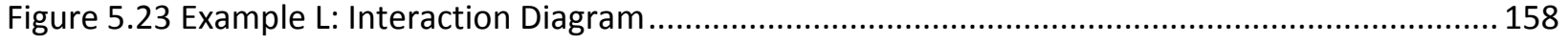




\section{List of Tables}

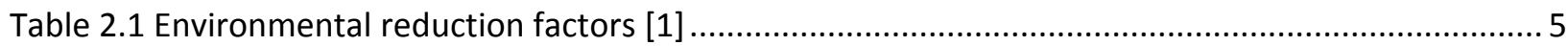

Table 2.2 Sustained Plus Cyclic Service Load Stress Limits in FRP Reinforcement ................................... 8

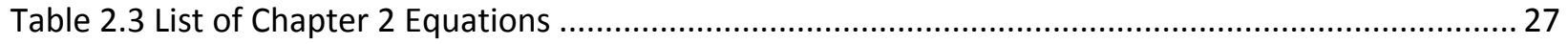

Table 4.1 Inputs for "UnstrengthenedCapacityOfUnstrengthenedColumn" ............................................58

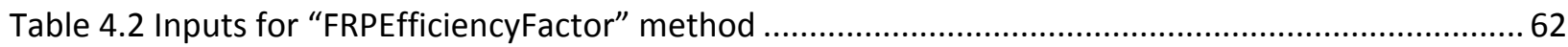

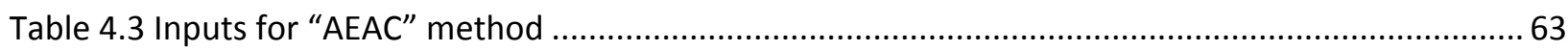

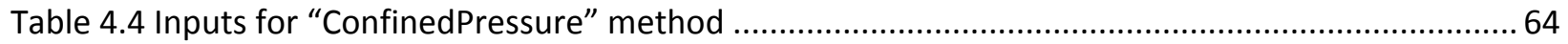

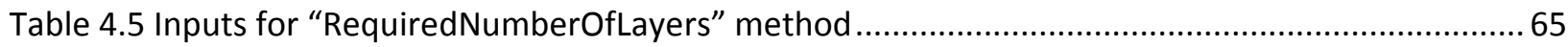

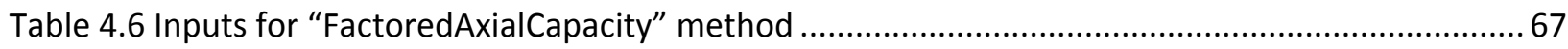

Table 4.7 Inputs for "CompressiveStrainOnConfinedColumn" method ...................................................69

Table 4.8 Inputs for "PointBorC" and "PointE" methods ........................................................................ 96

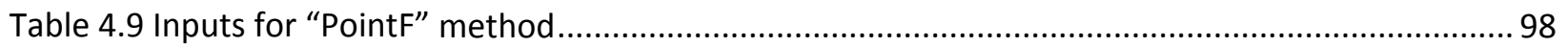

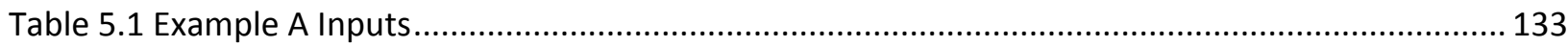

Table 5.2 Comparison of Example A outputs.................................................................................... 135

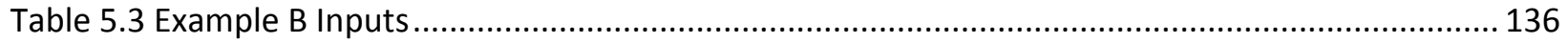

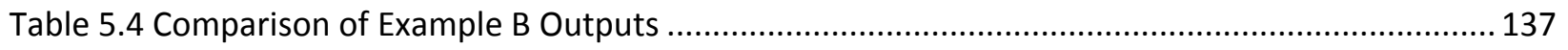

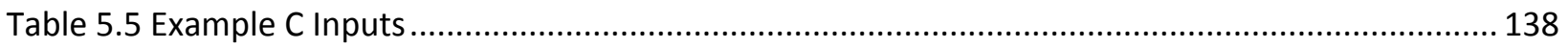

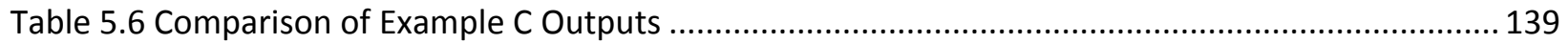

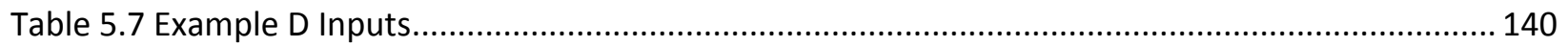

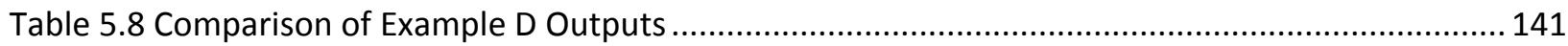

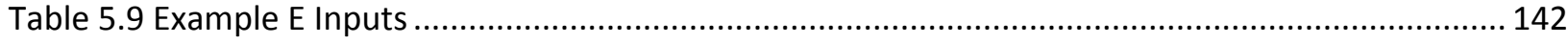

Table 5.10 Comparison of Example E Outputs …............................................................................. 143

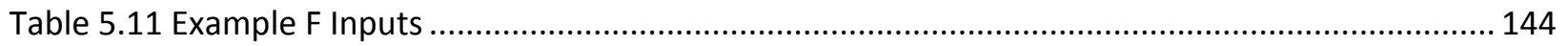

Table 5.12 Comparison of Example F Outputs............................................................................. 145

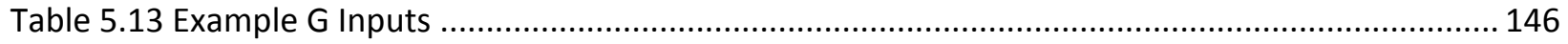

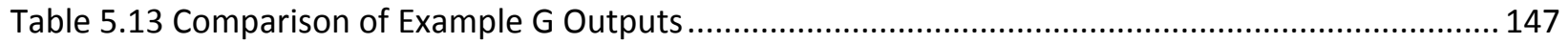

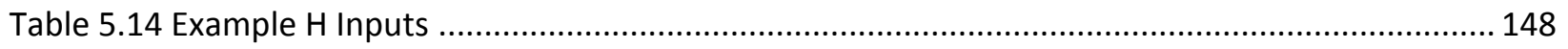

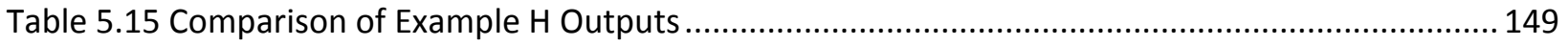


Table 5.16 Example I Inputs 150

Table 5.17 Comparison of Example I Outputs 151

Table 5.18 Example J Inputs. 152

Table 5.19 Comparison of Example I Outputs ... 153

Table 5.20 Example K Inputs 154

Table 5.21 Comparison of Example K Outputs 156

Table 5.22 Example L Inputs 157

Table 5.23 Comparison of Example L Outputs. .158 


\section{List of Equations}

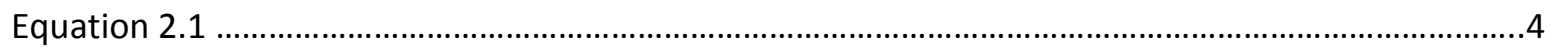

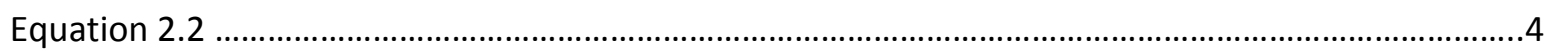

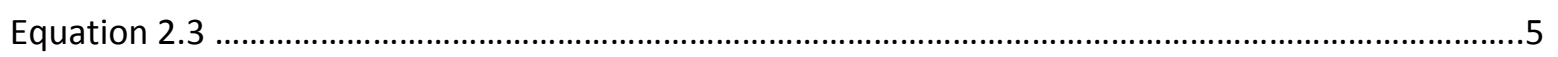

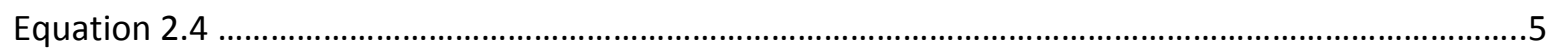

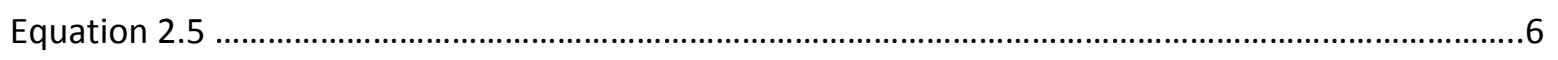

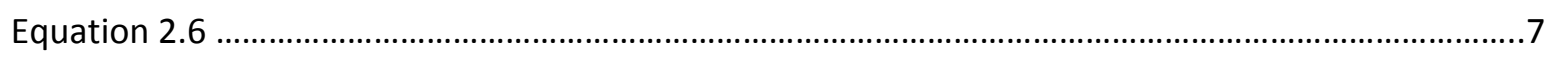

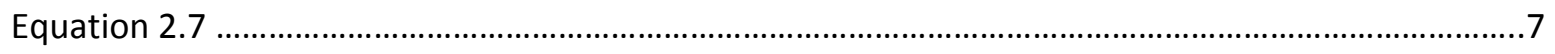

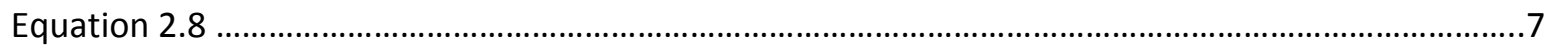

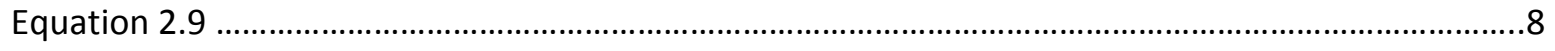

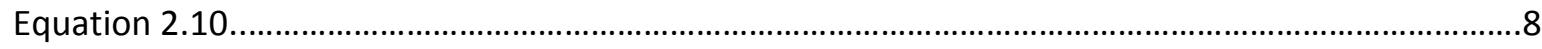

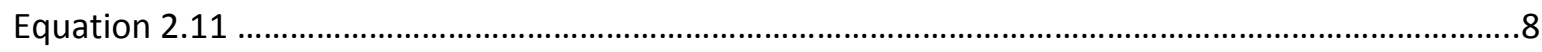

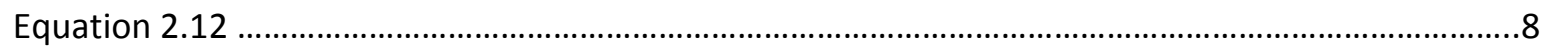

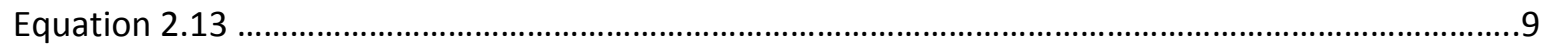

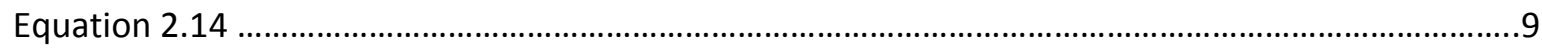

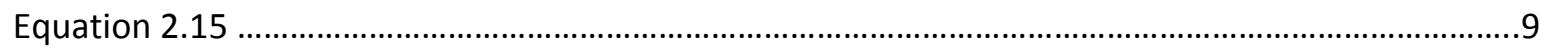

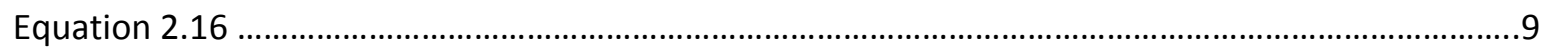

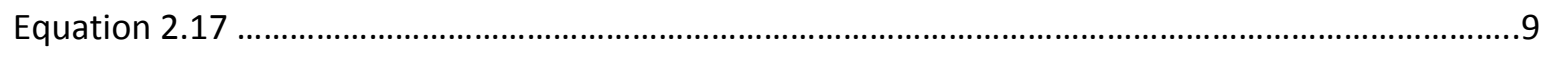

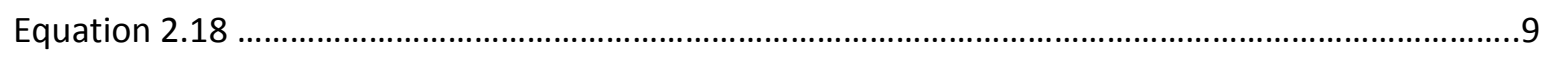

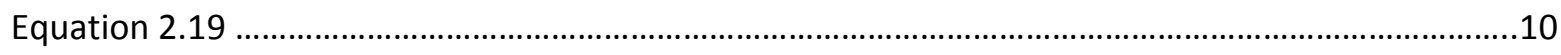

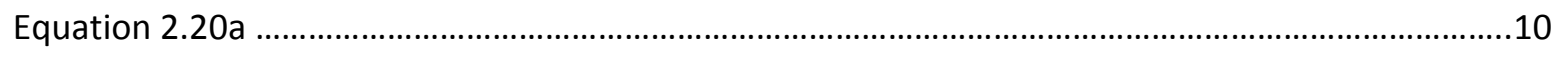

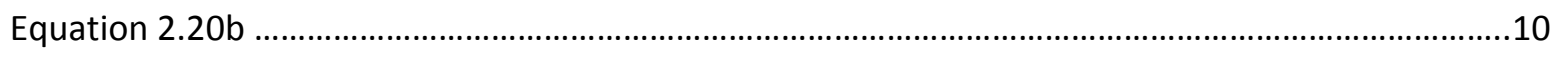

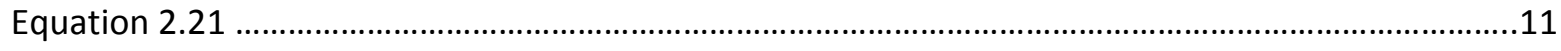

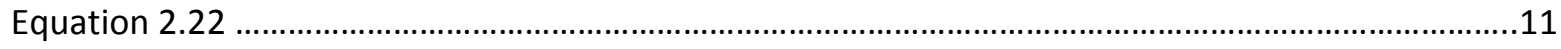




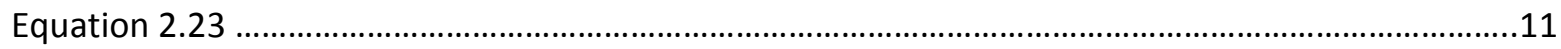

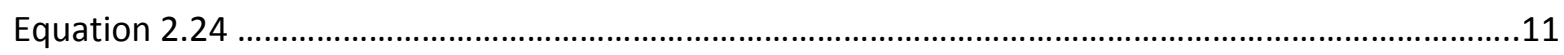

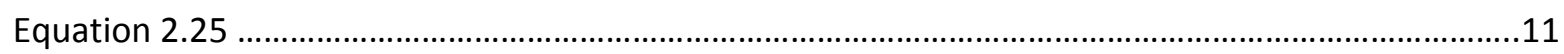

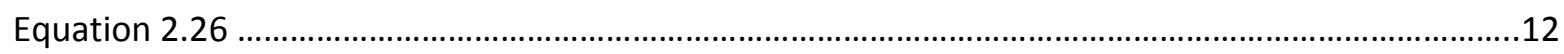

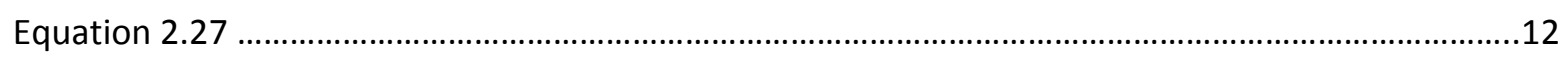

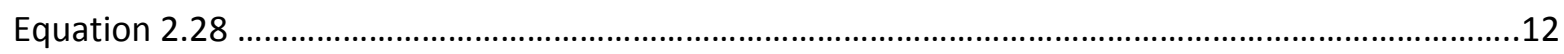

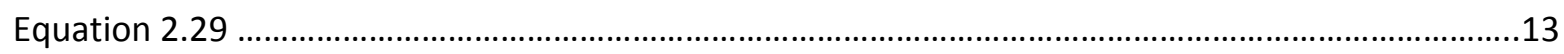

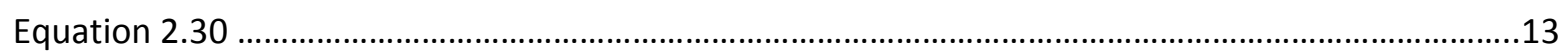

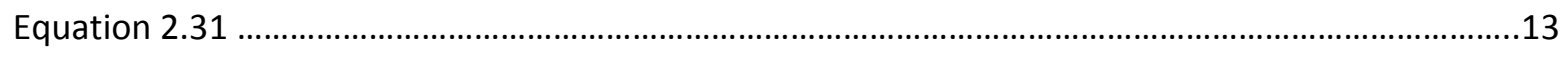

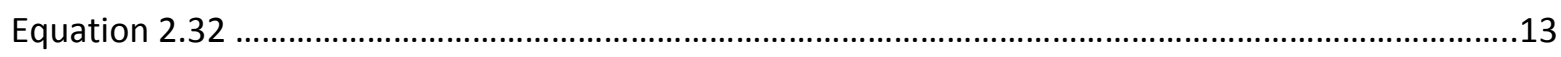

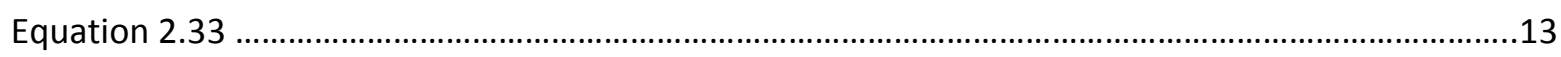

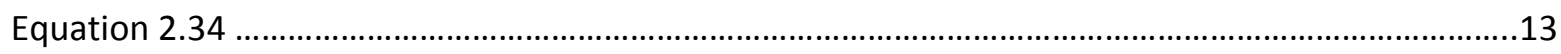

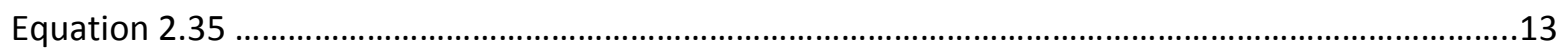

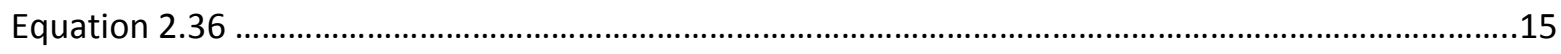

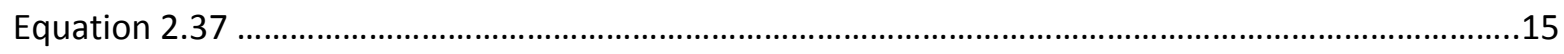

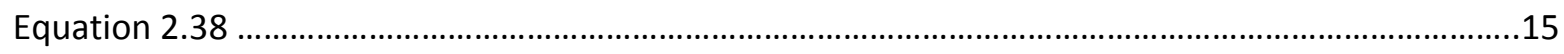

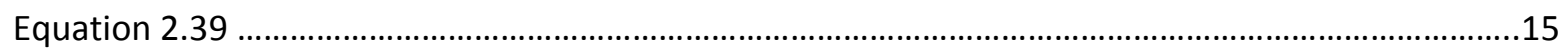

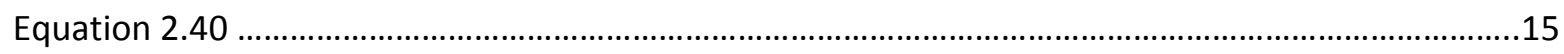

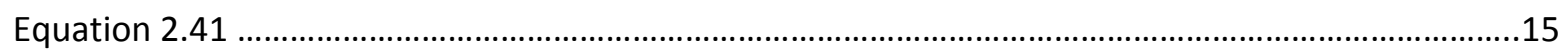

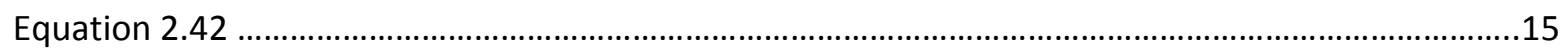

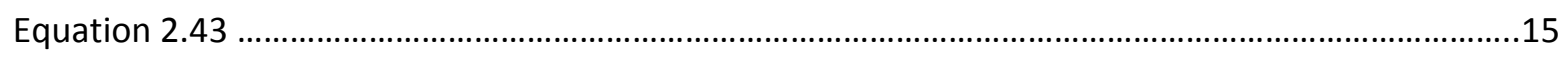

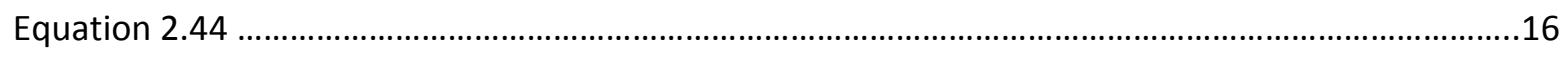

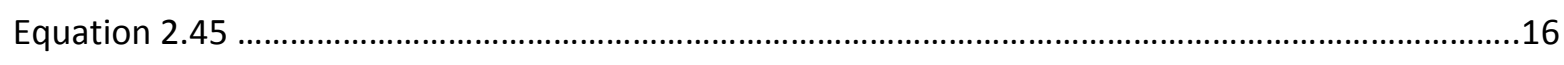

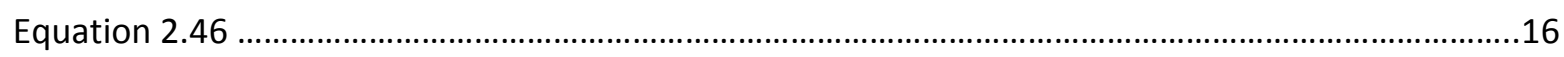

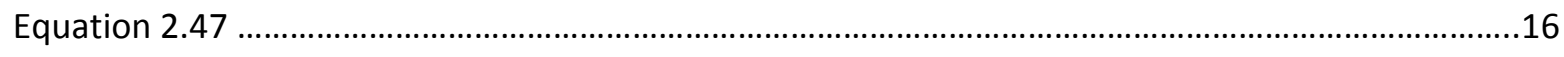




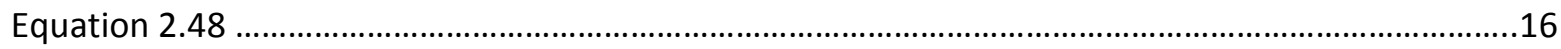

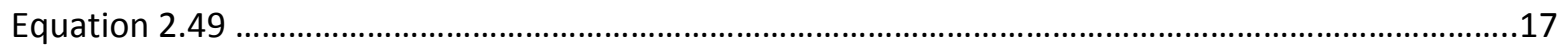

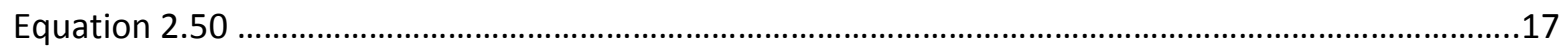

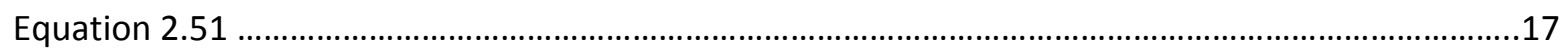

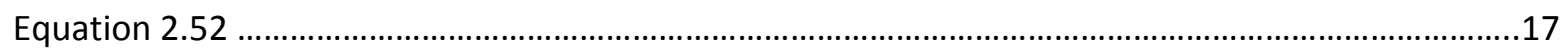

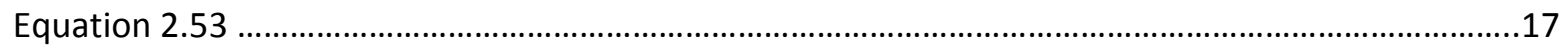

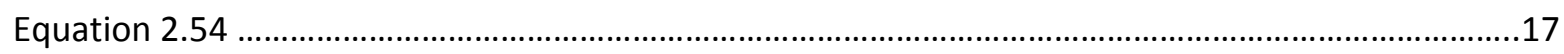

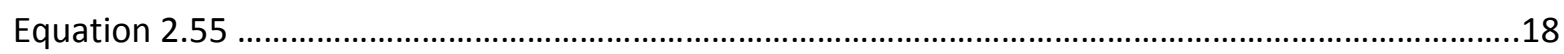

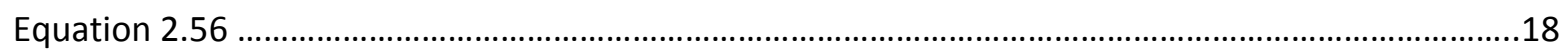

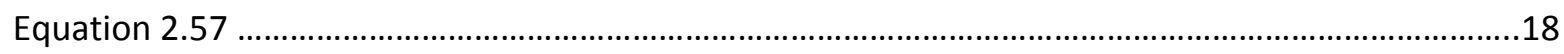

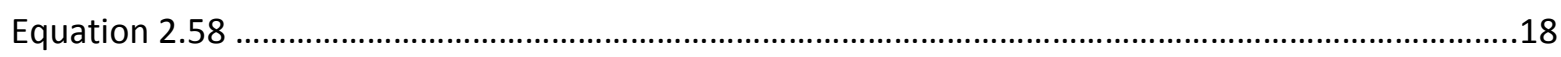

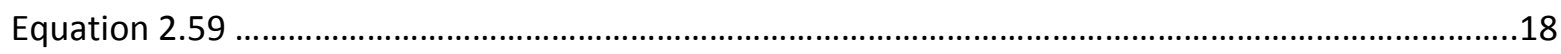

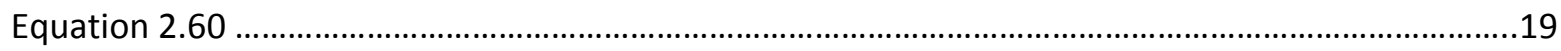

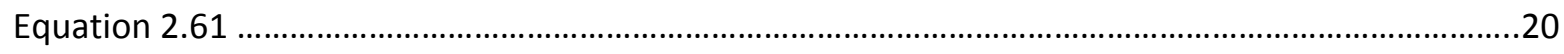

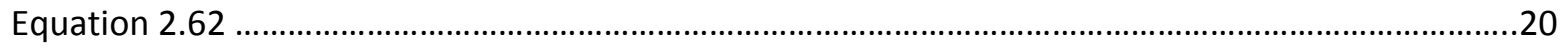

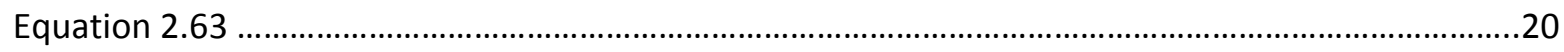

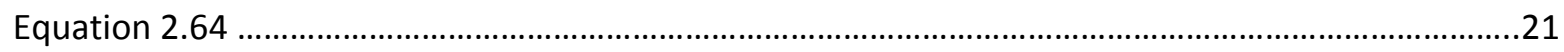

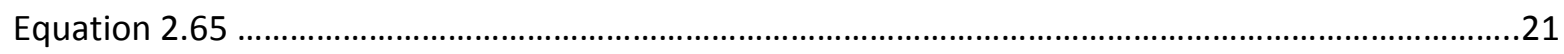

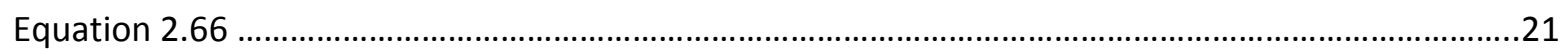

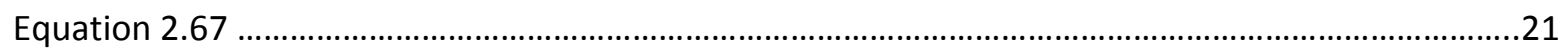

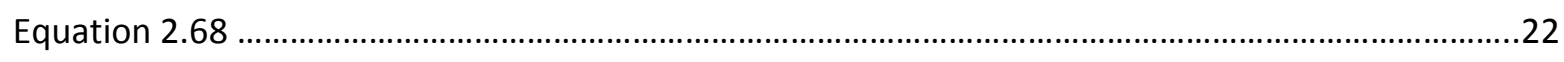

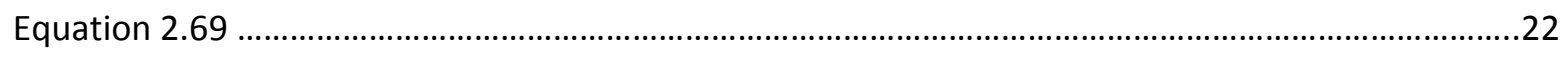

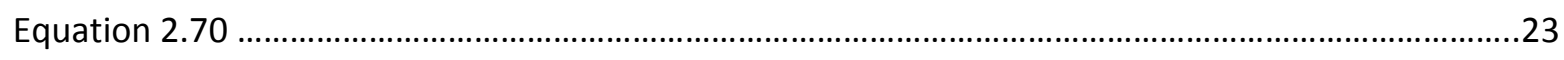

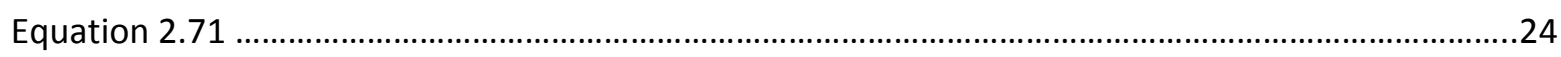

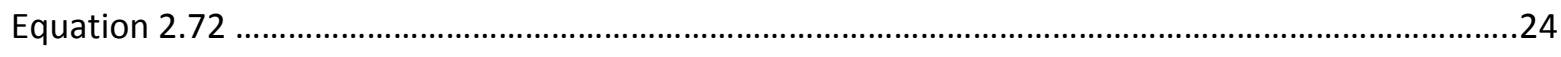




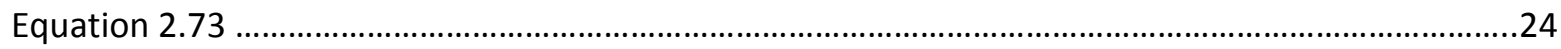

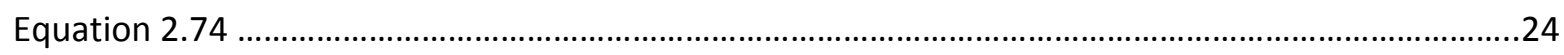

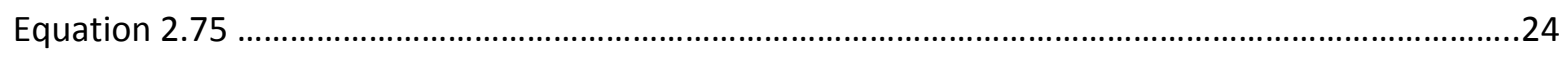

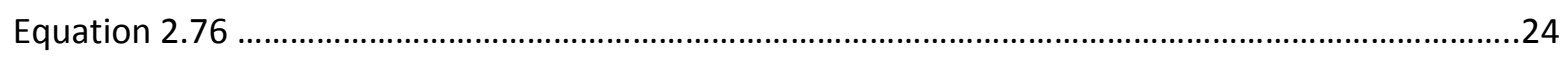

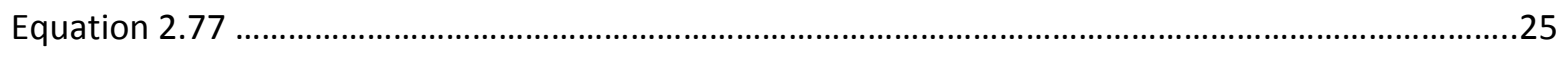

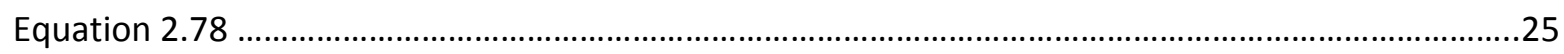

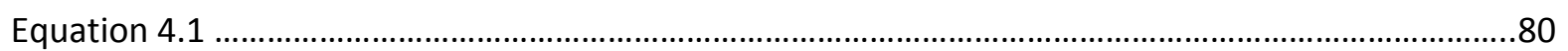




\section{Notation}

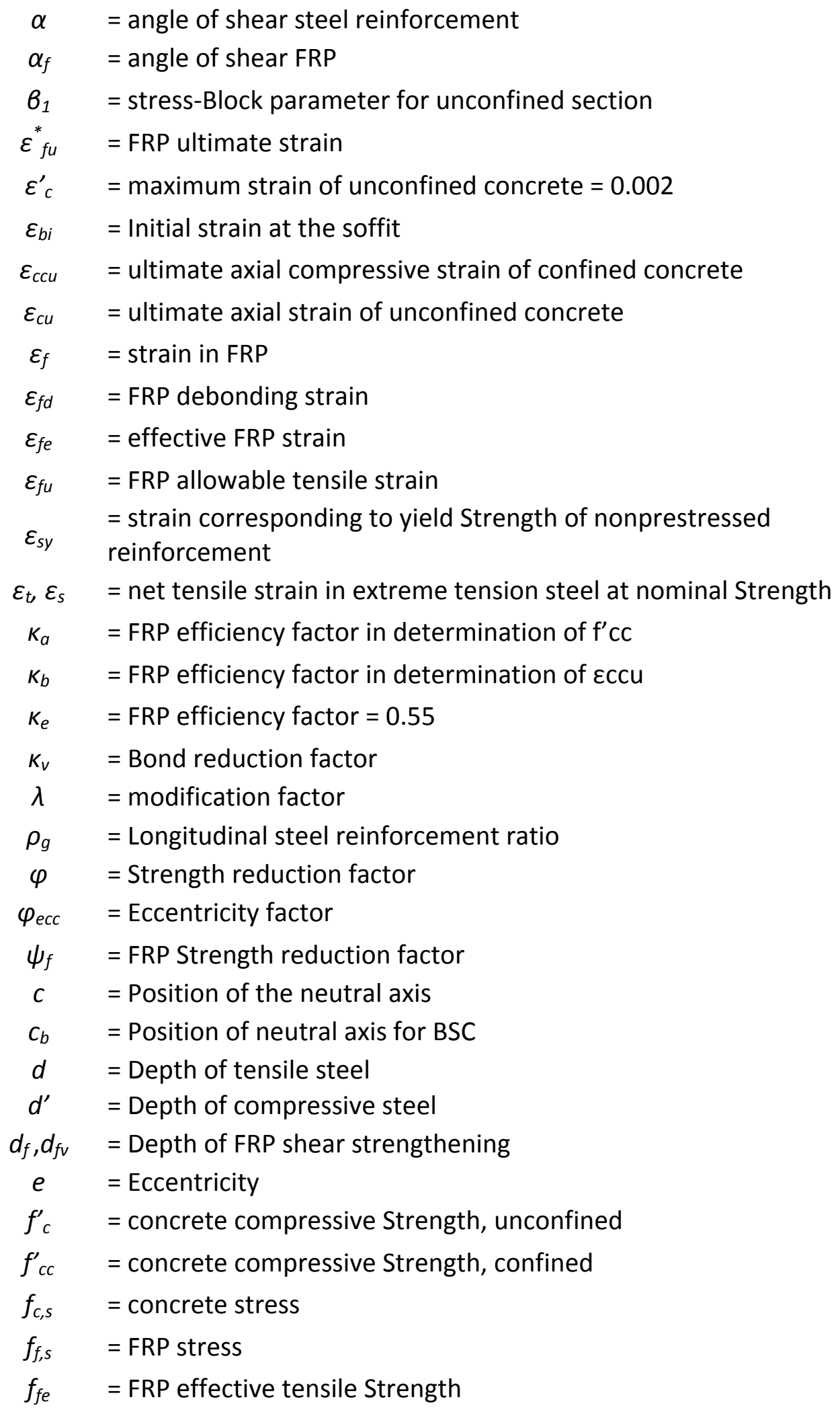




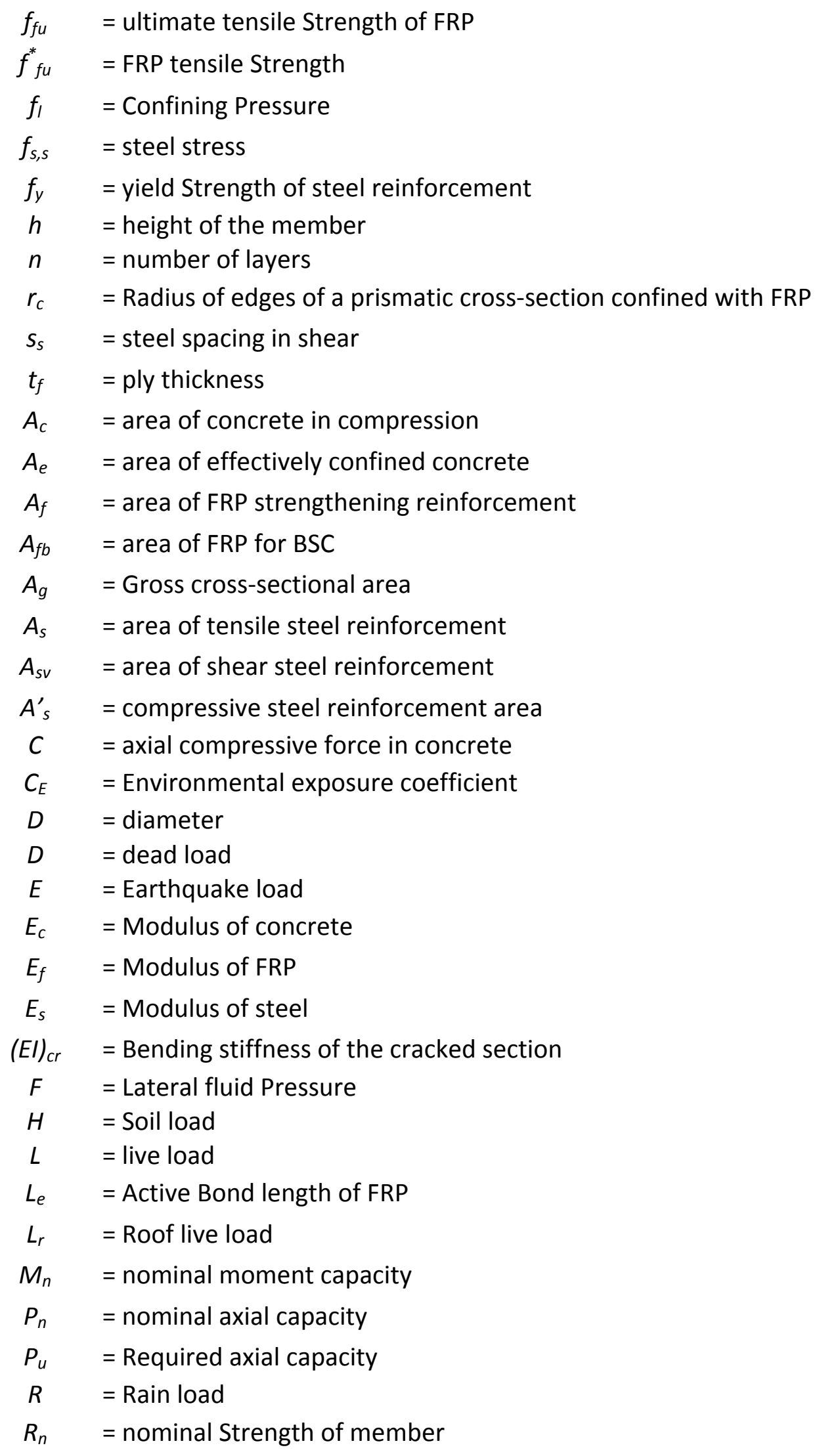




$$
\begin{array}{ll}
R_{S} & =\text { stress resultant } \\
S & =\text { Snow load } \\
S_{D L} & =\text { dead load effects } \\
S_{L L} & =\text { live load effects } \\
T & =\text { creep or shrinkage and temperature load } \\
T_{f} & =\text { tensile force in FRP } \\
T_{s} & =\text { tensile force in steel } \\
U & =\text { Required capacity } \\
V_{c} & =\text { shear capacity of concrete } \\
V_{f} & =\text { shear capacity of FRP } \\
V_{n} & =\text { nominal shear capacity } \\
V_{f}^{R} & =\text { Required shear capacity of FRP } \\
V_{s} & =\text { shear capacity of steel reinforcement } \\
W & =\text { Wind load }
\end{array}
$$




\section{Chapter 1 - Introduction}

\section{Introduction}

Fiber-reinforced polymers (FRPs) has proven to be an effective alternative to retrofitting existing reinforced concrete structures compared to more traditional means such as externally bonded steel plates, steel or concrete jackets, and external post-tensioning. Often using FRP is cheaper than traditional methods of repair even including the initial large expense of producing the FRP system. Also, in areas with difficult or limited access, FRP systems are easier to apply [1].

Because of benefits of using FRP systems to rehabilitate dilapidated or structurally deficient concrete structures, the supplemental software Computer Aided Design Environment for Composites (CADEC) for Introduction to Composites Materials Designs [4] needs an add-on application to the current analysis and design features specifically to complement Chapter 13 of the textbook. Chapter 13 discusses the analysis and design process for strengthening reinforced concrete beams and columns under flexure, shear, axial, or a combination of axial and flexure loading [4].

CADEC uses a database for storing what the software calls documents. These documents are where the user creates fibers, matrices, laminas, and laminates which store associated properties of the each item and can be used throughout the application. Therefore, before a straight add-on application can be developed and implemented, a proven method for semi- 
automating the design procedure for strengthening of reinforced concrete structures needs to be developed.

\subsection{Objective}

The purpose of this thesis is to develop methods that can be implemented in creating an application to design and verify strengthened reinforced concrete beams and columns. Therefore, two major components are required in order to make a method for design that not only works but also capable of being used by others to develop into an application.

The first component is the method, but the method must be developed in such a manner to allow for easy understanding and simple implementation. Because of this requirement, the methods are developed in the object-oriented programming language, $\mathrm{C \#}$ (pronounced "C sharp"). As for any object-oriented language, $\mathrm{C \#}$ uses classes which are composed of class methods. Therefore, the design methods are to be developed into class methods which are accessible when the class is available. For example, a programmer should be able to calculate the original neutral axis of the reinforced concrete beam without having to recreate the class method, but rather use the developed methods to simply call the class method to achieve the desired value.

The second component is to verify the methods do work. Therefore, a window application must be designed and operating using the class methods and the design process for strengthening. This application must run several cases and match manual design calculations.

\subsection{Organization}

Chapter 2 provides a review of the design procedure and governing specifications for strengthening reinforced concrete structures. A brief discussion on $\mathrm{ACl} 318$ [1] and $\mathrm{ACl} 440.2 \mathrm{R}$ [2] specifications is presented. Also Chapter 13 of Introduction to Composite Materials Design [4] is discussed in greater 
detail, including the design procedure for strengthening beams in flexure and shear, columns in pure axial and verification of beam-columns.

Chapter 3 explains how to use and what to expect from the windows form application. This chapter can be considered a user's guide to the application.

Chapter 4 discusses the developed method and the implementation of the methods in a windows form application. A general architecture of the application is provided as well as a traceability matrix to help follow the flow and relationship of different classes and forms throughout the application. First classes and the associated methods are discussed, followed by forms.

Chapter 5 compares the application outputs against hand calculations to validate the methods and demonstrate using the applications for all potential design paths.

Chapter 6 summarizes these comparisons and the developed methods, discuss general conclusions of the methods, and make further recommendations to improving and expanding the methods and windows form application. 


\section{Chapter 2 - Literature Review}

\subsection{ACl440.2R-08}

This section is an overview of the $\mathrm{ACl} 440.2 \mathrm{R}-08$ which is used to guide design and construction of externally bonded FRP systems for strengthening concrete structures. Only chapters relevant to the development of the software are discussed.

\subsubsection{Strengthening Limits}

Strengthening limits are used to prevent the structure from collapsing when the FRP reinforcement fails from damage or any other cause. The structure needs to hold a sufficient level of load so that repairs of the FRP system can be safely done [1].

$\left(\varphi R_{n}\right)_{\text {exisiting }} \geq\left(1.1 S_{D L}+0.75 S_{L L}\right)_{\text {new }}$

The minimum resistance of the unstrengthened structure must meet Eq. 2.1 which incorporates the factored anticipated dead load and live load of the new loading combinations [1].

\subsubsection{Environmental Reduction Factor}

Because environmental conditions can have adverse affects on FRP, an environmental reduction factor is used when determining such items as the tensile strength, rupture strain, and elastic modulus. The reduction factor varies on the type of fiber being considered and the environment the FRP system will be exposed, and can be seen in Table 2.1 [1].

Most material properties given by the manufacturers do not include long-term exposures. Therefore the ultimate strength is determined using the environmental reduction factor in Eq. 2.2 [1].

$f_{f u}=C_{E} f_{f u}^{*}$ 


\section{Table 2.1 Environmental reduction factors [1]}

\begin{tabular}{|c|c|c|}
\hline $\begin{array}{c}\text { Exposure } \\
\text { Conditions }\end{array}$ & $\begin{array}{c}\text { Fiber } \\
\text { Type }\end{array}$ & $\begin{array}{c}\text { Reduction } \\
\text { Factor }\end{array}$ \\
\hline \multirow{4}{*}{ Interior } & Carbon & 0.95 \\
\cline { 2 - 3 } & Glass & 0.75 \\
\cline { 2 - 3 } & Aramid & 0.85 \\
\hline \multirow{4}{*}{ Exterior } & Carbon & 0.85 \\
\cline { 2 - 3 } & Glass & 0.65 \\
\cline { 2 - 3 } & Aramid & 0.75 \\
\hline \multirow{4}{*}{ Aggressive } & Carbon & 0.85 \\
\cline { 2 - 3 } & Glass & 0.5 \\
\cline { 2 - 3 } & Aramid & 0.7 \\
\hline
\end{tabular}

The rupture strain is adjusted as well using Eq. 2.3 [1].

$\varepsilon_{f u}=C_{E} \varepsilon_{f u}^{*}$

Typically, unidirectional FRP behaves linear elastically, and therefore Hooke's law can be used to determine the modulus for FRP as seen in Eq. 2.4 [1].

$E_{f u}=f_{f u} / \epsilon_{f u}$

Eq. 2.4

\subsubsection{Flexural Strengthening}

\subsubsection{Failure Modes}

There are several failure modes that control the flexure strength of a FRP-strengthened section which include crushing of the concrete in compression before yielding of the steel reinforcement, the rupture of the FRP after the steel reinforcement yields in tension, the yielding of the reinforcing steel in tension followed by the crushing of the concrete, the delamination of the concrete cover by shear or tension, and FRP debonding from the concrete structure [1]. 
Crushing of concrete is assumed to occur when the compression strain reaches a value of 0.003 . The rupture of the FRP is assumed to occur when the strain reaches the designed rupture strain. The most concerning issue is cracked-induced debonding. To prevent this failure mode, the effective strain of the FRP needs to be limited to the strain value from Eq. 2.5. Also, a strength reduction factor for the FRP is introduced to address issues of reliability of the FRP [1].

$\varepsilon_{f d}=0.41 \sqrt{\frac{f^{\prime} c}{n E_{f} t_{f}}} \leq 0.9 \varepsilon_{f u}$

\subsubsection{Reinforced Concrete Member Assumptions}

$\mathrm{ACl}$ makes the following assumptions when calculating the flexural resistance of a strengthened reinforced concrete section using FRP [1].

- "Design Calculations are based on the dimensions, internal reinforcing steel arrangement, and material properties of the existing member being strengthened" [1];

- "The strains in the steel reinforcement and concrete are directly proportional to the distance from the neutral axis. This is, a plane section before loading remains plane after loading" [1];

- "There is no relative slip between external FRP reinforcement and the concrete" [1];

- "The shear deformation within the adhesive layer is neglected because the adhesive layer is very thin with slight variations in its thickness" [1];

- "The maximum usable compressive strain in the concrete is 0.003 " [1];

- "The tensile strength of the concrete is neglected" [1];

- "The FRP reinforcement has a linear elastic stress-strain relationship to failure" [1].

\subsubsection{Stress and Stain Levels in FRP Reinforcement}

The stress and strain levels are important to determine and compare to the ultimate limit states. The maximum strain is achieved at one of three situations, 1) the strain level of the FRP when the 
concrete crushes, 2) when the FRP ruptures, or 3) when the FRP reinforcement debonds from the concrete. The effective strain can be calculated using Eq. 2.6 [1].

$\varepsilon_{f e}=\varepsilon_{c u}\left(\frac{d_{f}-c}{c}\right)-\varepsilon_{b i} \leq \varepsilon_{f d}$

The effective stress of the FRP reinforcement can be determined using the effective strain since the FRP behaves in a linearly elastic manner as seen in Eq. 2.7 [1].

$f_{f e}=E_{f} \varepsilon_{f e}$

\subsubsection{Strength Reduction Factor}

The application of external FRP reinforcement does affect the ductility of the concrete beam and though loss is sometimes negligible, the situation of large losses must be addressed. The tension controlled state must be checked with accordance of Eq. 2.8 which has the strength reduction factor for ductile sections as 0.9 and for brittle sections as 0.65 with a linear transition for steel that has not yielded [1].

$\varphi=\left\{\begin{array}{c}0.9 \text { for } \varepsilon_{t} \geq 0.005 \\ 0.65+\frac{0.25\left(\varepsilon_{t}-\varepsilon_{s y}\right)}{.005-\varepsilon_{s y}} \text { for } \varepsilon_{s y}<\varepsilon_{t}<.005 \\ 0.65 \text { for } \varepsilon_{t} \leq \epsilon_{s y}\end{array}\right.$

\subsubsection{Serviceability}

To prevent excessive deformations of the reinforced concrete section with FRP reinforcement, the reinforcing steel should be prevented from yielding under services loads. "The stress in the steel reinforcement under service load should be limited to $80 \%$ of the yield strength as shown in" Eq. 2.9. "In addition, the compressive stress in concrete under service load should be limited to $45 \%$ of the compressive strength, as shown in" Eq. 2.10 [1]. 
$f_{s, s} \leq 0.8 f_{y}$

$f_{c, S} \leq 0.65 f^{\prime}{ }_{c}$

The stress levels in the FRP need to be limited to avoid creep-rupture or failure to cyclic stresses and fatigue. Cyclic service load stress limits for FRP reinforcement, Table 2.2, is dependent on fiber type, and the stress limit is expressed in Eq. 2.11 [1].

\section{Table 2.2 Sustained Plus Cyclic Service Load Stress Limits in FRP Reinforcement}

\begin{tabular}{|c|c|c|c|}
\hline & \multicolumn{3}{|c|}{ Fiber Type } \\
\hline Stress type & GFRP & AFRP & CFRP \\
\hline $\begin{array}{c}\text { Sustained plus } \\
\text { cyclic stress limit }\end{array}$ & $0.20 f_{f u}$ & $0.30 f_{f u}$ & $0.55 f_{f u}$ \\
\hline
\end{tabular}

$f_{f, s} \leq$ sustained plus cyclic stress limit

Eq. 2.11

\subsubsection{Nominal Flexure Strength}

The nominal flexure strength of a reinforced concrete section with external FRP reinforcement is equated using Eq. 2.12. The reduction factor for FRP is applied with the recommended value of 0.85 [1].

$M_{n}=A_{s} f_{s}\left(d \frac{\beta_{1} c}{2}\right)+\psi_{f} A_{f} f_{f e}\left(h \frac{\beta_{1} c}{2}\right)$

\subsubsection{Shear Strengthening}

\subsubsection{Completely Wrapped members}

A reinforced concrete member can be wrapped completely, therefore all sides of the section have externally applied FRP reinforcement, but this wrapping scheme is known to produce a failure mode where there is a loss of aggregate interlock of the concrete before the ultimate fiber strain is achieved. To prevent this failure mode, the maximum strain of the design is limited to $0.4 \%$ when 
members are completely wrapped, Eq. 2.13. Higher strains should not be used for FRP shear strengthening [1].

$\varepsilon_{f e}=.004 \leq 0.75 \epsilon_{f u}$

\subsubsection{Bonded U-wraps or Bonded Face Plies}

Reinforced concrete sections that have bonded U-wraps (threes side have applied FRP reinforcement) or sections having bonded face plies (FRP applied to only the two vertical faces of the sections) delaminate before there is a loss of aggregate interlock in the concrete. Therefore, the effective strain is calculated using a shear bond-reduction factor, Eq. 2.14 [1].

$\epsilon_{f e}=\kappa_{v} \varepsilon_{f u} \leq .004$

The bond reduction factor is calculated using equations 2.15 to 2.18 and is a function of the concrete strength and the type of wrapping scheme being used [1].

$\kappa_{v}=\frac{k_{1} k_{2} L_{e}}{11,900 \varepsilon_{f u}} \leq 0.75$

$L_{e}=\frac{23,300}{\left(n_{f} t_{f} E_{f}\right)^{0.58}}$

$k_{1}=\left(\frac{f^{\prime} c}{27}\right)^{2 / 3}$

$k_{2}=\left\{\begin{array}{c}\frac{d_{f v}-L_{e}}{d_{f v}} \text { for } U-\text { wraps } \\ \frac{d_{f v}-2 L_{e}}{d_{f v}} \text { for two sides bonded }\end{array}\right.$

The active bond length calculated in Eq. 2.16 is the length over which the majority of the bond stress is maintained [1]. 


\subsubsection{Shear Spacing}

The spacing limitations for FRP strips for shear strengthening adhere to the same limits in $\mathrm{ACl}$ 318 for internal steel shear reinforcement which states that for nonprestressed members the spacing shall not exceed half of the depth or 24 inches $(610 \mathrm{~mm})$. The spacing is from the centerline of one FRP strip to the next [1].

\subsubsection{Reinforcement Limits}

The sum of the contributed strength of the FRP reinforcement and steel shear reinforcement is taken as the total shear strength. The total shear reinforcement is limited based $\mathrm{ACl} 318$ for steel. Eq. 2.19 gives the shear limit [2].

$V_{s}+V_{f} \leq 0.66 \sqrt{f^{\prime}}{ }_{c} b d$

\subsubsection{Axial Force Strengthening}

\subsubsection{Pure Axial Compression Strength}

The axial compression strength of a non-slender concrete column that is confined by externally placed FRP reinforcement is calculated using Eq. 2.20. The reduction factor should be included based on the spiral and tie reinforcement [1].

For members with steel spiral reinforcement, Eq. 2.20a is used. For members with steel tie reinforcement, Eq. 2.20b is used [1].

$$
\begin{aligned}
& \varphi P_{n}=0.85 \varphi\left[0.85 f^{\prime}{ }_{c c}\left(A_{g}-A_{s}\right)+f_{y} A_{s}\right] \\
& \varphi P_{n}=0.80 \varphi\left[0.85 f^{\prime}{ }_{c c}\left(A_{g}-A_{s}\right)+f_{y} A_{s}\right]
\end{aligned}
$$

The confined concrete compressive strength is found using Eq. 2.21 and the confined pressure caused by the applied FRP reinforcement is determined from Eq. 2.22. The confined compressive 
strength includes an additional reduction factor for the FRP reinforcement which is suggested to be 0.95 , and an efficiency factor to account for the geometry of the section. The confinement ratio of confined concrete section (the confined pressure divided by the unconfined compressive strength) has to be equal to or greater than $0.08[1]$.

$f^{\prime}{ }_{c c}=\psi_{f} 3.3 \kappa_{a} f_{l}$

$f_{l}=\frac{2 E_{f} n t_{f} \varepsilon_{f e}}{D}$

The efficiency factor, or shape factor, is calculated based on the cross-sectional area of the effectively confined concrete area and the side aspect ratio. For circular cross-sections, the reinforcement provides a uniformed confined pressure, and therefore the efficiency factor is 1.0. When the cross-section is non-circular, the efficiency factor is calculated using Eq. 2.23. For determining the confined pressure of a non-circular section, the diameter is assumed to be the diagonal of the rectangular section, expressed in Eq. 2.24 [1].

$\kappa_{a}=\frac{A_{e}}{A_{c}}\left(\frac{b}{h}\right)^{2}$

$D=\sqrt{b^{2}+h^{2}}$

The ratio of the effectively confined area of concrete expressed in the efficiency factor is found in Eq. 2.25 as a function of the radius of the corners and the longitudinal steel reinforcement ratio [1].

$\frac{A_{e}}{A_{c}}=\frac{1-\frac{\left[\left(\frac{b}{h}\right)\left(h-2 r_{c}\right)^{2}+\left(\frac{h}{b}\right)\left(b-2 r_{c}\right)^{2}\right]}{3 A g}-\rho_{g}}{1-\rho_{g}}$ 


\subsubsection{Stress and Strain Limits}

To prevent the loss of concrete integrity from excessive cracking, the compressive strain in the confined concrete section can be found using Eq. 2.26 and is limited to 0.01 . An efficiency factor takes into account the geometry of the cross-section and is calculated in Eq. 2.27 [1].

$\epsilon_{c c u}=\varepsilon^{\prime}{ }_{c}\left(1.5+12 \kappa_{b} \frac{f_{l}}{f^{\prime} c}\left(\frac{\epsilon_{f e}}{\epsilon_{c}^{\prime}}\right)^{0.45}\right)$

$\kappa_{b}=\frac{A_{e}}{A_{c}}\left(\frac{b}{h}\right)^{0.5}$

To ensure that significant cracking of the column does not occur, service loads should not induced such conditions, and the FRP jacket should only need to contain the damage during conditions that are excessive and temporary. Therefore, the concrete strain should remain below the concrete cracking strain, which means that the compressive stress of the concrete should be limited to $65 \%$ of the compressive strength. Also, the longitudinal steel reinforcement should avoid plastic deformation during sustained and cyclical loading, and therefore the service stress should be below $60 \%$ of the yield strength of steel [1].

The effective strain of the FRP jacket should also be limited to ensure shear integrity and prevent premature failure of the FRP system caused by multi-axial stress or cracking of the concrete, which produce concentrated regions of stress. Eq. 2.28 expresses the limitation of the effective strain of the FRP jacket where the strain efficiency factor is 0.55 [1].

$\epsilon_{f e}=0.004 \leq \kappa_{e} \varepsilon_{f u}$ 


\subsection{Loading Combinations}

According to $\mathrm{ACl} 318$, the required strength of a member is determined using factored loads where each load factor assigned "is influenced by the degree of accuracy of which the load effect usually can be calculated and the variation that might be expected in the load during the lifetime of the structure." Also, some factors are assigned to a specific combination of loads because of the probability of simultaneous occurrence. Equations 2.29 through 2.35 are the given loading combinations [2].

$$
\begin{aligned}
& U=1.4(D+F) \\
& U=1.2(D+F+T)+1.6(L+H)+.5\left(L_{r} \text { or } S \text { or } R\right) \\
& U=1.2 D+1.6\left(L_{r} \text { or } S \text { or } R\right)+(L \text { or } 0.8 W) \\
& U=1.2 D+1.6 W+L+.5\left(L_{r} \text { or } S \text { or } R\right) \\
& U=1.2 D+E+L+0.2 S \\
& U=0.9 D+1.6 W+1.6 H \\
& U=0.9 D+E+1.6 H
\end{aligned}
$$

\subsection{Flexure Strengthening}

\subsubsection{Unstrengthened Behavior}

An unstrengthened reinforced concrete member is a member without FRP. The behavior of the unstrengthened section of reinforced concrete is virtually linear. During initial loading, flexure cracks of the concrete on the tension surface begin to develop when the stress reaches the tension strength of the concrete. Once cracking occurs, the tensile force is transferred to the steel. Once the steel stress has reached the yield strength, the reinforced concrete structure will redistribute the forces. If the steel yields before the concrete crushes, the response is ductile, cracking patterns in the concrete are evident, 
and the beam experiences considerable deflections before failure. If the reinforced concrete member has a significant amount of steel reinforcement, therefore requiring more loading to yield, the concrete will crush before yielding of the steel occurs, causing failure. This is known as brittle failure [4].

\subsubsection{Strengthened Behavior}

When FRP is applied, the reinforced concrete member is considered to be strengthened. At first, the stiffness of the member is basically unchanged to the unstrengthened behavior. This is because the applied FRP strips are very thin and do not cause any notable changes in the stiffness of the structure, but behavior is affected. The tension force is shared with the steel and FRP reinforcement after the concrete has cracked, and FRP contribution for tension force is more relevant since it is located the farthest from the neutral axis. After the steel yields, the FRP is forced to carry the load the steel was carrying. The strengthened reinforced concrete member collapses at higher levels because of the FRP but observes lower deflections compared to the unstrengthened member, making the member less ductile [4].

In addition to the steel and FRP yielding before the concrete crushes, strengthened reinforced concrete members may be affected by other failure modes. The FRP is capable of failing due to delamination of the FRP to the concrete [4].

\subsubsection{Failure Modes}

Because of limitations of the concrete, steel reinforcement, and the FRP, the failure mode need to be determined. First possible failure mode for strengthened reinforced concrete member is the FRP failing before or after the steel reinforcement yields but prior to the concrete crushing. This failure mode is known as weak strengthening configuration (WSC). The other failure mode to be considered is when the concrete crushes on the compression side of the member before or after the yielding of the steel reinforcement. This is called strong strengthening configuration (SSC) [4]. 
Also, there is a stage known as the balanced strengthening configuration (BSC) which is used for preliminary design purposes. The BSC case is when the failure of the concrete and FRP occurs simultaneously and can be either elastic or inelastic depending on if the steel has or has not yielded [4].

\subsubsection{Strong Strengthening Configuration (SSC)}

In SSC, the section fails from concrete crushing or reaching strains of 0.003 , and the FRP strain does not reach its effective strain. Eqs 2.36 to 2.38 are used to define the strain relationships for the SSC [4].

$\varepsilon_{c}=\varepsilon_{c u}=.003$

$\varepsilon_{f}=\varepsilon_{c u} \frac{h-c}{c}-\varepsilon_{b i}<\varepsilon_{f e}$

$\varepsilon_{s}=\varepsilon_{c u} \frac{d-c}{c}$

The neutral axis is calculated by balancing the tension and compression forces as seen in Eq.

2.39 and solving the system using the quadratic formula by substituting Eq. 2.40-2.44 into Eq. 2.39. Use

Eq. 2.42 or 2.43 if the steel does or does not yield [4].

$C-T_{s}-T_{f}=0$

$C=0.85 f^{\prime}{ }_{c} \beta_{1} c b$

$T_{f}=E_{f} A_{f} \epsilon_{f}$

$T_{s}=A_{s} f_{y}$ if $\epsilon_{s} \geq \epsilon_{y}$

$T_{s}=A_{s} E_{s} \epsilon_{s}$ if $\varepsilon_{s}<\epsilon_{y}$ 
$\beta_{1}=\left\{\begin{array}{c}0.85 \text { if } f^{\prime}{ }_{c} \leq 27.57 \mathrm{mPa} \\ 0.85-.05\left(f^{\prime}{ }_{c}-27.57\right) / 6.9 \text { if } 27.57 \mathrm{mPa}<f^{\prime}{ }_{c} \leq 55.1 \mathrm{mPa} \\ 0.65 \text { if }{f^{\prime}}^{\prime}>55.15 \mathrm{mPa}\end{array}\right.$

Eq. 2.44

\subsubsection{Weak Strengthening Configuration (WSC)}

When the FRP fails before the concrete is crushed, also known as WSC, the FRP strain is equal to the effective stain and the concrete strain is always below the ultimate concrete compressive strain of .003. The strain relationships are defined in Eqs. 2.41-2.43 [4].

$\varepsilon_{c}=\left(\varepsilon_{f e}-\varepsilon_{b i}\right) \frac{c}{h-c} \leq \varepsilon_{c u}$

$\varepsilon_{f}=\varepsilon_{f e}$

$\varepsilon_{s}=\left(\epsilon_{f e}-\epsilon_{b i}\right) \frac{d-c}{h-c}$

Similarly to the SSC, the neutral axis is solved by balancing the tension and compression forces in the section, Eq. 2.39, and using the quadratic equation with Eqs. 2.40-2.42 and Eq. 2.48 for when the steel does not yield [4].

$T_{s}=A_{s} E_{s}\left(\epsilon_{f e}-\epsilon_{b i}\right) \frac{d-c}{h-c}$ if $\varepsilon_{s}<\epsilon_{y}$

\subsubsection{Balanced Strengthening Configuration (BSC)}

The balanced strengthening configuration occurs when the concrete and FRP failure happens simultaneously. This configuration is used for design purposes because it utilizes both materials effectively, but to achieve this state is usually not practicable because the area of FRP needed does not match what is common in marketplace [4]. 
Since the strain requirements of BSC are known, the neutral axis is calculated as a function of the concrete and FRP strain values, Eq. 2.49, and the strain in the steel reinforcement is determined using Eq. 2.50

$c_{b}=\frac{\epsilon_{c u} h}{\varepsilon_{c u}+\varepsilon_{f e}+\epsilon_{b i}}$

$\epsilon_{s}=\frac{\epsilon_{c u}}{c_{b}}\left(d-c_{b}\right)$

With the neutral axis known, the required area of FRP can be determined using Eq. 2.39, and Eq. 2.51 is used when the steel does not yield [4].

$T_{s}=A_{s} E_{s} \frac{\epsilon_{c u}}{c_{b}}\left(d-c_{b}\right)$ if $\varepsilon_{s}<\epsilon_{y}$

By rearranging Eq. 2.39, an estimate for the required area of FRP needed to reach equilibrium can calculated by Eq. 2.52 or Eq. 2.53 when the steel yields or does not yield, respectively [4].

$A_{f b}=\frac{1}{E_{f} \epsilon_{f e}}\left(0.85 f^{\prime}{ }_{c} \beta_{1} b c_{b}-A_{s} f_{y}\right)$

$A_{f b}=\frac{1}{E_{f} \epsilon_{f e}}\left(0.85 f^{\prime}{ }_{c} \beta_{1} b c_{b}-A_{s} E_{s} \frac{\epsilon_{c u}}{c_{b}}\left(d-c_{b}\right)\right)$

\subsubsection{Serviceability Limit States}

To meet $\mathrm{ACl}$ stress limitations, three serviceability limit states are considered in determining the stress levels of the concrete, FRP, and steel reinforcement. The first to be considered is the general case where all three are present in the strain distribution. The neutral axis is calculated by using Eq. 2.39 and after substituting the strain relationships of the FRP and steel, Eq. 2.54 [4].

$E_{c} b c^{2}-E_{s} A_{s}(d-c)-E_{f} A_{f}(h-c)=0$ 
To determine the stress values of the FRP, steel, and concrete, the moment capacity for service loading is used along with the bending stiffness of the cracked section given by Eq. 2.55. The stress of the concrete, steel, and FRP is calculated using Eq. 2.56, Eq. 2.57, and Eq. 2.58 respectively [4].

$(E I)_{c r}=\frac{E_{c} b c^{3}}{3}+E_{s} A_{s}(d-c)^{2}+E_{f} A_{f}(h-c)^{2}$

$f_{c}=E_{c} M c /(E I)_{c r}$

$f_{s}=E_{s} M(d-c) /(E I)_{c r}$

$f_{f}=E_{f} M(h-c) /(E I)_{c r}$

The second state to be considered is the original configuration, which is before the FRP is applied. The neutral axis is determined by taking Eq. 2.54 and solving when the area of the FRP is zero, $A_{f}=0$. The stress of the steel and concrete are calculated by using Eq. 2.56 and 2.57 after calculating the cracked stiffness, Eq. 2.55, with the area of FRP equal to zero. It must be noted that the moment capacity for this state only takes into account dead loads and reduced live loads [4].

The initial strain can also be calculated by Eq. 2.59. The initial strain is determined the surface of the section where the FRP is planned to be applied [4].

$\varepsilon_{b i}=\frac{M_{D L}\left(h-c_{A}\right)}{(E I)_{c r}}$

The last state is when the member is strengthened and using the serviceability loading. The neutral axis is determined using Eq. 2.54 taking into account the applied FRP reinforcement. The stress of the concrete and steel are calculated using Eq. 2.56 and 2.57 using the supplementary moment due to the serviceability loading combinations [4]. 
Finally the stress of the concrete and steel are determined by adding the stress values of the second and third states. The stress of the FRP occurring in the third state is calculated by using Eq. 2.60 [4].

$f_{f}=f_{s}\left(\frac{E_{f}}{E_{S}}\right) \frac{h-c_{B}}{d-C_{B}}-\varepsilon_{b i} E_{f}$

\subsubsection{Design Procedure}

This section will provide a brief overview of the design procedure for strengthening reinforced concrete beams for flexure.

The first step is to determine the initial moment capacity of the beam and compare it to minimum required capacity. Also, the maximum allowable reinforcement of the original section is calculated. Second, the initial strain of the soffit beam as well as the stress distribution is calculated while only considering dead loads. Next the FRP system is selected and preliminary values are calculated using the environmental reduction factor [4].

Having the original capacity and the initial strain, an approximate area of FRP can be calculated using Eq. 2.61. With an estimate of the area of FRP, determine the actual area of FRP to be applied and the corresponding configuration. Determine the capacity of the strengthen system and verify it meets the required capacity. If it does not, increase the area of FRP until the strengthened beam satisfies the required capacity [4].

Lastly, the serviceability requirements must be verified. 


\subsection{Shear Strengthening}

Reinforced concrete members can be further strengthened for shear loading to prevent failure of shear and shear cracking. FRP is applied externally to the faces of the concrete member to achieve the increased shear strength. Strengthened shear reinforced concrete configurations include continuous and discontinuous schemes, where the beam is usually completely wrapped, but only where it is considered necessary. To prevent the beam from debonding or delamination, the strain limits in section 2.1.4 are used [4].

The shear capacity of the unstrengthened reinforced concrete is calculated using Eq. 2.61, which is a function of the compressive strength of the concrete and the cross-sectional dimensions [4].

$V_{c}=\lambda \frac{b d}{6} \sqrt{f^{\prime}}$

Between continuous and discontinuous wrapping, discontinuous is the preferred method of strengthening because it does not capture moisture which could assist in debonding and delamination. The nominal shear capacity of the beam is calculated by adding the capacities of each material, as seen in Eq. 2.62 [4].

$V_{n}=V_{c}+V_{s}+\psi V_{f}$

\subsubsection{Discontinuous Wrapping}

The shear capacity of the steel reinforcement of the strengthened section when the FRP is stripped along the length of the beam in intermittent distances can be determined using Eq. 2.63 . It is a function of the distance between reinforcement and the angle of the steel reinforcement [4].

$V_{s}=f_{y} A_{s v} \frac{d}{s_{s}}[\sin \alpha+\cos \alpha]$ 
The FRP strip's shear capacity can be calculated using Eq. 2.64 which also a function of the applied angle of the FRP, as well as the distance of the strips [4].

$V_{f}=f_{f e}\left(2 n_{f} t_{f} w_{f}\right) \frac{d_{f v}}{s_{f}}\left[\sin \alpha_{f}+\cos \alpha_{f}\right]$

\subsubsection{Continuous Wrapping}

When the applied FRP reinforcement is layered along the length of the beam, the applied angles of the steel and FRP reinforcement are assumed to be $90^{\circ}$. Therefore the shear steel capacity is calculated by Eq. 2.65 and the FRP capacity is calculated using Eq. 2.66 [4].

$V_{s}=f_{y} A_{s v} \frac{d}{s_{s}}$

$V_{f}=f_{f e}\left(2 n_{f} t_{f} w_{f}\right) d_{f v}$

\subsubsection{Design Procedure}

In order to determine the number of layers needed to meet the required shear capacity of the beam, the initial shear capacity of the beam must be determined. That is when the beam is unstrengthened and all the shear capacity comes from the concrete and steel stirrups. The initial capacity is then compared to the minimum required strength and the maximum allowable strengthening governed by $\mathrm{ACl} 440[4]$.

With initial capacities, an estimate of the number of layers is obtained based on the design scheme, continuous, discontinuous, complete wrapping, three-sided, or two-sided. By rearranging Eq. 2.66 and Eq. 2.62, the number of layers can be determined as seen in Eq. 2.67 where the nominal capacity is replaced by the required capacity [4].

$n_{f}=\frac{1}{\psi_{f}}\left[\frac{V_{u}}{\phi}-V_{c}-V_{s}\right] \frac{1}{f_{f e}\left(2 t_{f} w_{f}\right) d_{f v}}$ 
After determining the number of layers to be applied to the reinforced concrete beam, the shear capacity of the FRP can be calculated and the limit for total shear reinforcement needs to be verified [4].

\subsection{Beam-Column Strengthening}

Beam-columns can be strengthened by confining the reinforced concrete with FRP which increases the confined compression strength of the concrete. A beam-column's initial behavior for the confined column is very similar to the unconfined reinforced concrete column. Not until the column has cracked does the FRP show influence. The stress-strain relationship of the post-cracked confined column behaves linearly because of the stiffness of the FRP reinforcement [4].

\subsubsection{Pure Axial Compression Design Procedure}

First the required loading capacity and the unconfined axial capacity of the column are calculated so that the minimum required capacity and the maximum allowable strengthening can be checked [4].

With the required capacity known, the confined concrete strength is calculated by rearranging Eq. 2.20 to solve for the confined concrete strength with the nominal capacity replaced with the required loading capacity as seen in Eq. 2.68 [4].

$f^{\prime}{ }_{c c}=\frac{1}{0.85\left(A_{g}-A_{s}\right)}\left(\frac{P_{u}}{\phi \phi_{e c c}}-f_{y} A_{s}\right)$

To determine the number of required layers of FRP, the confined pressure is needed. By using the value of confined concrete strength from Eq. 2.68, Eq. 2.21 can be rearranged to determine the confined pressure (Eq. 2.69). Finally the number of layers is calculated by using Eq. 2.70 [4].

$f_{l}=\frac{f^{\prime} c c^{-}-f^{\prime} c}{\varphi_{f} 3.3 \kappa_{a}}$ 
$n=\frac{D f_{l}}{2 t_{f} E_{f} \epsilon_{f e}}$

The confined capacity of the column is recalculated using the number of layers used, and compared to the required capacity. If the required capacity is still less than the confined capacity, additional layers should be used until the capacity is met. Lastly, the serviceability requirements need to be checked [4].

\subsubsection{Beam-Column Verification Procedure}

To verify that a confined reinforced concrete column meets the required loading capacity, an interaction diagram is developed by calculating capacities of the beam-column at significant junctures of the diagram [4].

Prior to any critical values being calculated, several preliminary values are needed which include the ratio of the effective confined area over the area of concrete, the effective strain limit, efficiency factors, the confined pressure, the confined concrete compressive strength, the confined compressive strain, and the confined stress-block factors [4].

The first point to be calculated is point A in Figure 2.1 where the only axial loading is considered. Therefore the axial compressive capacity is calculated using Eq. 2.20 for strengthened and unstrengthened columns. Point $\mathrm{D}$ is considered next. Point $\mathrm{D}$ is the where an $\mathrm{ACl}$ introduced eccentricity of $0.1 \mathrm{~h}$ is considered along with the nominal axial capacity [4].

Point $B$ is the first point that considers the stress-strain relationship of the beam-column. At point $B$, the extreme compressive fiber is equal to the ultimate confined compression strain and the strain of the steel reinforcement closest to the tensile fiber of the beam-column is assumed to be zero. Therefore, in order to calculate the axial and moment capacity of the beam-column, the neutral axis and the stress resultants of the steel reinforcement needs to be calculated for each layer of steel 
reinforcement in the beam-column. Eq. 2.71 can be used to determine the neutral axis for any given steel strain. The steel strain is calculated with respect of the distance of the steel reinforcement to the compression fiber of the member as seen in Eq. 2.72, and the stress resultant for the steel reinforcement is calculated using Eq. 2.73 [4].

$c=\frac{\varepsilon_{c}}{\varepsilon_{c}+\varepsilon_{s n}} d$

$\epsilon_{s i}=\frac{\varepsilon_{c}}{c}\left(d_{i}-c\right)$

$f_{s i}=\left\{\begin{array}{c}f_{y} \text { if } \epsilon_{s i} \geq \varepsilon_{y} \text { and } \varepsilon_{s i} \leq-\epsilon_{y} \\ E_{s} \epsilon_{s i} \text { if }-\varepsilon_{y}<\varepsilon_{s i}<\varepsilon_{y}\end{array}\right.$

The stress resultants for the concrete and steel reinforcement are calculated by Eq. 2.40 and Eq. 2.74 , respectively. With the stress resultants, the nominal axial and moment capacities can be calculated with Eq. 2.75 and Eq. 2.76 [4].

$R_{s i}=f_{s i} A_{s i}$

$P_{n}=C-\sum_{i}^{n_{s}} R_{s i}$

$M_{n}=P_{n} e=C\left(\frac{h}{2}-\beta_{1} \frac{c}{2}\right)+\sum_{i}^{n_{s}} R_{s i}\left(d_{i}-\frac{h}{2}\right)$

Point $\mathrm{C}$ of the interaction diagram is when the assumed strain distribution has the steel reinforcement yielding and the concrete crushing simultaneously. Therefore the concrete strain is equal to the ultimate concrete strain and the steel strain equals the yield strain. The neutral axis is given by Eq. 2.71 and the steel strain is given by Eq. 2.72. Point $C$ uses the same process of calculating the stress resultants, the values calculated for strain and the neutral axis by using Eq. 2.74 , which is used to determine the axial and moment capacity of the beam-column, Eq. 2.76 and 2.77 [4]. 
The next value in the interaction diagram is Point $E$. Point $E$ uses a fixed value of eccentricity corresponding to the intersection of a line equal to $45^{\circ}$ with the interaction diagram. This is also considered when the eccentricity is equal to $100 \mathrm{~mm}$, but the position of the neutral axis is not known; therefore, an iterative process is used to produce the eccentricity value of 100 by starting with an arbitrary value for the neutral axis. With a neutral axis, the same procedure as used with Point $B$ and $C$ is used to calculate the capacities. With the capacities, an eccentricity is determined and the percentage error is determined to see how close to 100 the value is. If the error is too great, then the neutral axis is changed appropriately, and the process is repeated until the error is within an acceptable range [4].

The last value of the interaction diagram is where only pure flexure is considered, Point $\mathrm{F} . \mathrm{ACl}$ only allows unstrengthened cases to be considered for Point $F$, and thus only steel reinforcement is considered in the moment capacity, this includes steel on the compression side. The neutral axis is calculated using Eq. 2.77 which includes the compression steel [4].

$0.85 f^{\prime}{ }_{c} \beta_{1} b c+A^{\prime}{ }_{s} E_{s} \varepsilon_{c u}\left(\frac{c-d^{\prime}}{c}\right)-A_{s} f_{y}=0 \quad$ (modified)

The moment capacity of the unconfined beam-column is determined using Eq. 2.78 [4].

$M_{n}=A_{s} f_{y}\left(d-\beta_{1} \frac{c}{2}\right)+A^{\prime}{ }_{s} E_{s} \varepsilon_{c u}\left(\frac{c-d^{\prime}}{c}\right)\left(\beta_{1} \frac{c}{2}-d^{\prime}\right) \quad$ (modified)

Eq. 2.77 and Eq. 2.78 are modified equations of Barbero Eq. 13.41 and Eq. 13.47, respectively because the compression steel reinforcement affects the location of the neutral axis and the nominal moment capacity.

With all six points calculated for strengthened and unstrengthened cases, the interaction diagram (Figure 2.1) can be plotted and then used to verify the required axial and moment capacities 
are within the acceptable region. If not, a new design must be selected and then verified using the same process [4].

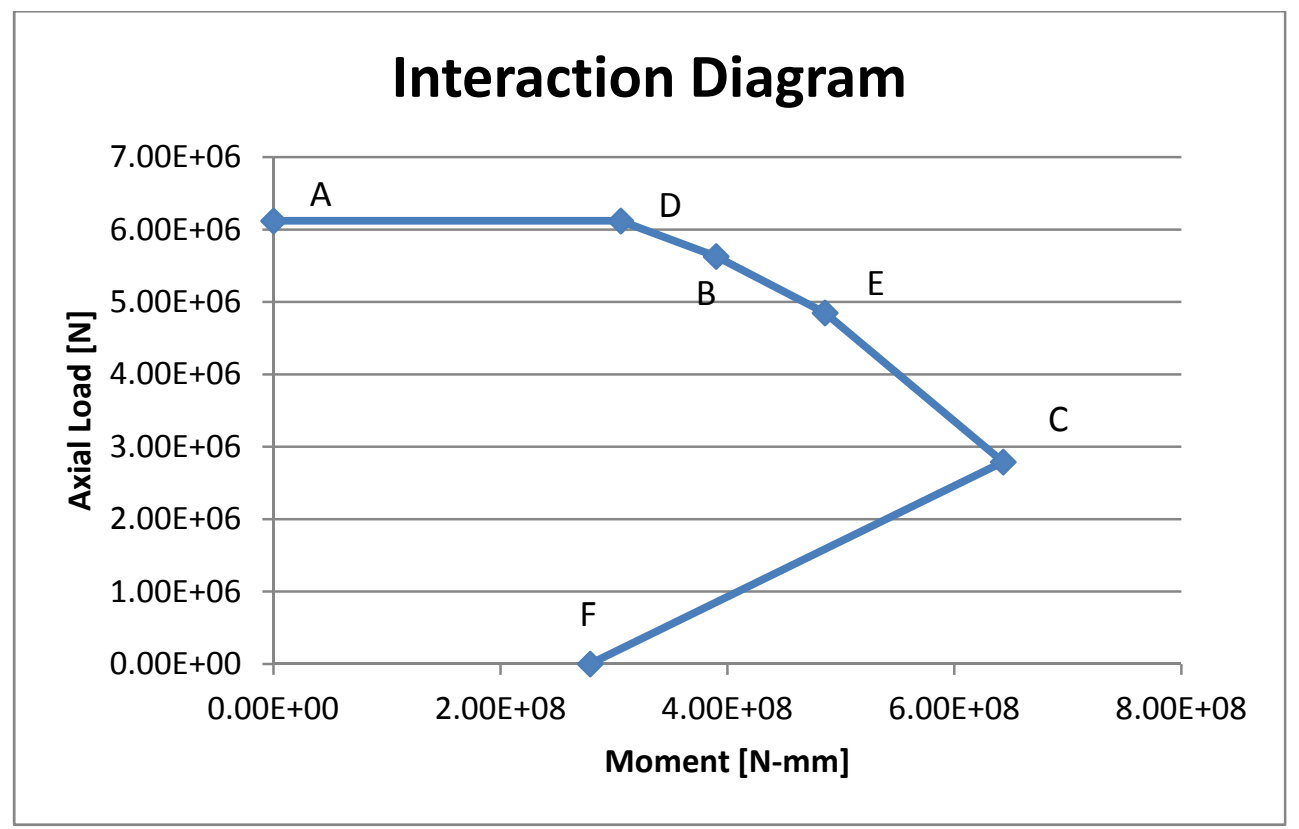

Figure 2.1 Interaction Diagram 
Table 2.3 List of Chapter 2 Equations

\begin{tabular}{|c|c|}
\hline Chapter 2 Equation & Equation Source \\
\hline Eq. 2.1 & $\mathrm{ACl} 440.2 \mathrm{R}$ Eq. 9-1 \\
\hline Eq. 2.2 & $\mathrm{ACl} 440.2 \mathrm{R}$ Eq. 9-3 \\
\hline Eq. 2.3 & $\mathrm{ACl} 440.2 \mathrm{R}$ Eq. 9-4 \\
\hline Eq. 2.4 & $\mathrm{ACl} 440.2 \mathrm{R}$ Eq. 9-5 \\
\hline Eq. 2.5 & $\mathrm{ACl} 440.2 \mathrm{R}$ Eq. $10-2$ \\
\hline Eq. 2.6 & $\mathrm{ACl} 440.2 \mathrm{R}$ Eq. $10-3$ \\
\hline Eq. 2.7 & $\mathrm{ACl} 440.2 \mathrm{R}$ Eq. $10-4$ \\
\hline Eq. 2.8 & $\mathrm{ACl} 440.2 \mathrm{R}$ Eq. $10-5$ \\
\hline Eq. 2.9 & $\mathrm{ACl}$ 440.2R Eq. 10-6 \\
\hline Eq. 2.10 & $\mathrm{ACl} 440.2 \mathrm{R}$ Eq. $10-7$ \\
\hline Eq. 2.11 & $\mathrm{ACl} 440.2 \mathrm{R}$ Eq. $10-8$ \\
\hline Eq. 2.12 & $\mathrm{ACl} 440.2 \mathrm{R}$ Eq. $10-13$ \\
\hline Eq. 2.13 & $\mathrm{ACl} 440.2 \mathrm{R}$ Eq.11-6a \\
\hline Eq. 2.14 & $\mathrm{ACl} 440.2 \mathrm{R}$ Eq. $11-6 \mathrm{~b}$ \\
\hline Eq. 2.15 & $\mathrm{ACl} 440.2 \mathrm{R}$ Eq. $11-7$ \\
\hline Eq. 2.16 & $\mathrm{ACl} 440.2 \mathrm{R}$ Eq. $11-8$ \\
\hline Eq. 2.17 & $\mathrm{ACl} 440.2 \mathrm{R}$ Eq. $11-9$ \\
\hline Eq. 2.18 & $\mathrm{ACl} 440.2 \mathrm{R}$ Eq. $11-10$ \\
\hline Eq. 2.19 & $\mathrm{ACl}$ 440.2R Eq.11-11 \\
\hline Eq. 2.20 & $\mathrm{ACl} 440.2 \mathrm{R}$ Eq. $12-1$ \\
\hline Eq. 2.21 & $\mathrm{ACl} 440.2 \mathrm{R}$ Eq. $12-3$ \\
\hline Eq. 2.22 & $\mathrm{ACl} 440.2 \mathrm{R}$ Eq. $12-4$ \\
\hline Eq. 2.23 & $\mathrm{ACl} 440.2 \mathrm{R}$ Eq. $12-9$ \\
\hline Eq. 2.24 & $\mathrm{ACl} 440.2 \mathrm{R}$ Eq.12-8 \\
\hline Eq. 2.25 & $\mathrm{ACl} 440.2 \mathrm{R}$ Eq.12-11 \\
\hline Eq. 2.26 & $\mathrm{ACl} 440.2 \mathrm{R}$ Eq. $12-6$ \\
\hline Eq. 2.27 & $\mathrm{ACl} 440.2 \mathrm{R}$ Eq. $12-10$ \\
\hline Eq. 2.28 & $\mathrm{ACl} 440.2 \mathrm{R}$ Eq. $12-12$ \\
\hline Eq. 2.29 & $\mathrm{ACl} 318$ Eq. 9-1 \\
\hline Eq. 2.30 & $\mathrm{ACl} 318$ Eq. 9-2 \\
\hline Eq. 2.31 & $\mathrm{ACl} 318 \mathrm{Eq} .9-3$ \\
\hline Eq. 2.32 & $\mathrm{ACl} 318$ Eq. 9-4 \\
\hline Eq. 2.33 & $\mathrm{ACl} 318 \mathrm{Eq} .9-5$ \\
\hline Eq. 2.34 & $\mathrm{ACl} 318$ Eq. 9-6 \\
\hline Eq. 2.35 & $\mathrm{ACl} 318$ Eq. 9-7 \\
\hline Eq. 2.36 & Barbero Eq. 13.16 \\
\hline Eq. 2.37 & Barbero Eq. 13.17 \\
\hline Eq. 2.38 & Barbero Eq. 13.18 \\
\hline Eq. 2.39 & Barbero Eq. 13.19 \\
\hline Eq. 2.40 & Barbero Eq. 13.24 \\
\hline Eq. 2.41 & Barbero Eq. 13.21 \\
\hline Eq. 2.42 & Barbero Eq. 13.22 \\
\hline Eq. 2.43 & Barbero Eq. 13.23 \\
\hline
\end{tabular}




\begin{tabular}{|c|c|}
\hline Eq. 2.44 & Barbero Eq. 13.25 \\
\hline Eq. 2.45 & Barbero Eq. 13.33 \\
\hline Eq. 2.46 & Barbero Eq. 13.34 \\
\hline Eq. 2.47 & Barbero Eq. 13.35 \\
\hline Eq. 2.48 & Barbero Eq. 13.40 \\
\hline Eq. 2.49 & Barbero Eq. 13.48 \\
\hline Eq. 2.50 & Barbero Eq. 13.49 \\
\hline Eq. 2.51 & Barbero Eq. 13.54 \\
\hline Eq. 2.52 & Barbero Eq. 13.55 \\
\hline Eq. 2.53 & Barbero Eq. 13.56 \\
\hline Eq. 2.54 & Barbero Eq. 13.64 \\
\hline Eq. 2.55 & Barbero Eq. 13.67 \\
\hline Eq. 2.56 & Barbero Eq. 13.66 \\
\hline Eq. 2.57 & Barbero Eq. 13.66 \\
\hline Eq. 2.58 & Barbero Eq. 13.66 \\
\hline Eq. 2.59 & Barbero Eq. 13.71 \\
\hline Eq. 2.60 & Barbero Eq. 13.77 \\
\hline Eq. 2.61 & Barbero Eq. 13.86 \\
\hline Eq. 2.62 & Barbero Eq. 13.95 \\
\hline Eq. 2.63 & Barbero Eq. 13.90 \\
\hline Eq. 2.64 & Barbero Eq. 13.91 \\
\hline Eq. 2.65 & Barbero Eq. 13.93 \\
\hline Eq. 2.66 & Barbero Eq. 13.94 \\
\hline Eq. 2.67 & Barbero Eq. 13.94-95 \\
\hline Eq. 2.68 & Barbero Eq. 13.113 \\
\hline Eq. 2.69 & Barbero Eq. 13.114 \\
\hline Eq. 2.70 & Barbero Eq. 13.115 \\
\hline Eq. 2.71 & Barbero Eq. 13.126 \\
\hline Eq. 2.72 & Barbero Eq. 13.119 \\
\hline Eq. 2.73 & Barbero Eq. 13.125 \\
\hline Eq. 2.74 & Barbero Eq. 13.125 \\
\hline Eq. 2.75 & Barbero Eq. 13.117 \\
\hline Eq. 2.76 & Barbero Eq. 13.118 \\
\hline Eq. 2.77 & Barbero Eq. 13.41 modified \\
\hline Eq. 2.78 & Barbero Eq. 13.47 modified \\
\hline
\end{tabular}




\section{Chapter 3 - Windows Forms Application}

\subsection{Introduction}

In order to make a functional user friendly program, a Windows Form application was produced, which functions as a supplement to Chapter 4 for preliminary design for the strengthening of reinforced concrete members. The application handles three different member types: beams, columns, and beamcolumns. This chapter will demonstrate the capabilities of the Windows Form application and serve as a user's manual.

\subsection{Program Overview}

The application has been organized by member type with concrete beams being broken down even further by strengthening design which includes flexure and shear strengthening. Once the user selects the desired member type, a series of window forms will follow requiring the inputs such as loading scenarios, geometric properties, material properties, and such. Once the user has filled all required fields, the final window form will display results and inform the user of the final outcome of the preliminary design.

\subsubsection{Main Form}

When the application is opened, the first form is the Main Form. The Main Form is the beginning of every path within the program and also where paths restart if the user back tracks. The form is rather simple as seen in Figure 3.1. The main and only purpose of the Main Form is to determine which member type the user will be strengthening, and does this by having three buttons each representing a member type. All the user must do is click on a member type. The application will close the Main Form and open a new form depending on the path chosen by the user. If the user clicks on the "Close" button, the Main Form closes and the application ends. 


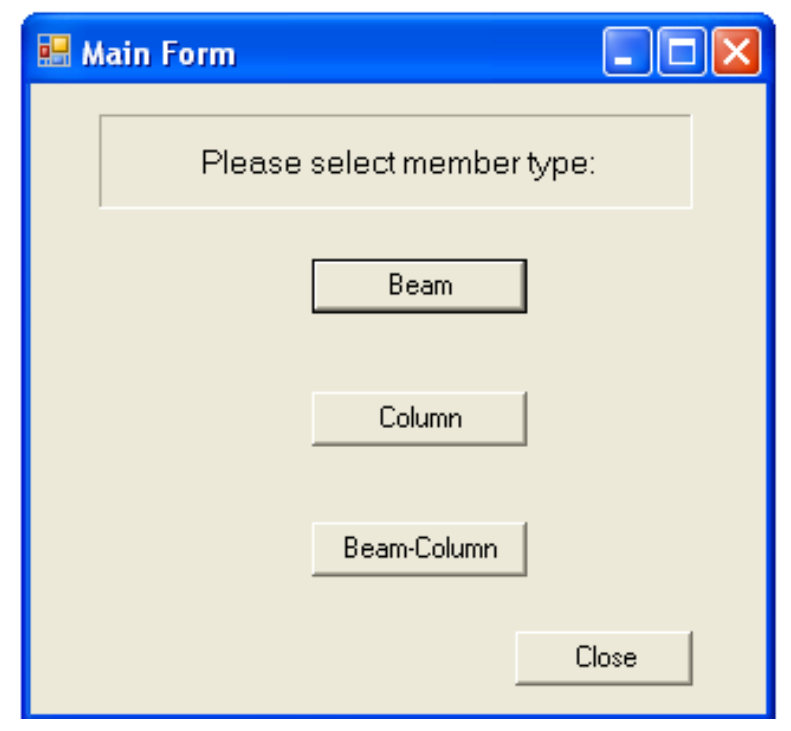

Figure 3.1 Main Form

\subsubsection{Strengthening of Beams}

If the user clicks the button "Beam," the Main Form closes followed by the Beam Form opening. The Beam Form is very similar to the Main Form with its formatting and simplicity. The Beam Form only purpose is to determine if the user wants to strengthen a beam in flexure or a beam in shear, hence the two buttons as seen in Figure 3.2. Each button closes the current form and opens a new form depending on the user selection. The "Back" button closes the Beam Form and returns the user to the Main Form where the user can restart the process. 


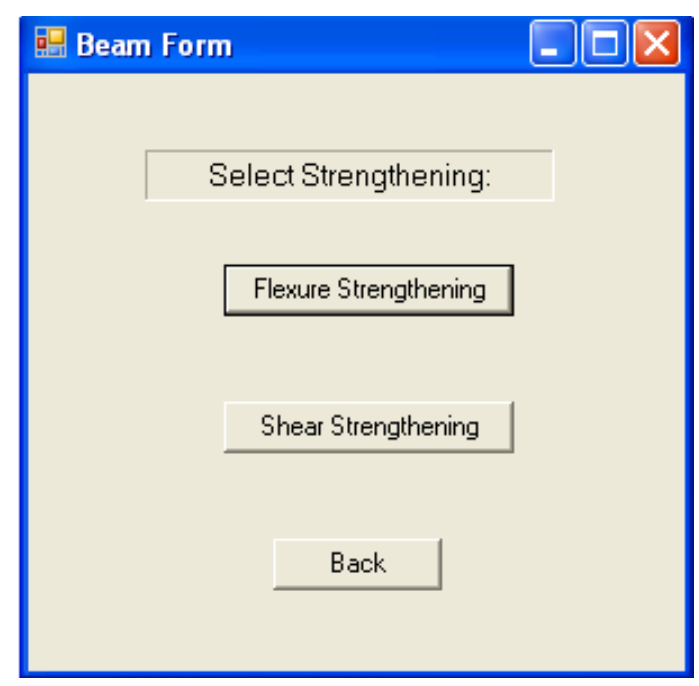

Figure 3.2 Beams Form

\subsubsection{Strengthening of Beams in Flexure}

If the user selects beams in the Main Form and flexure strengthening in the Beam Form, the program will now only follow the flexure strengthening path with produces three forms in succession. The first being the Loading Scenario form, followed by a form for providing the required properties for the design process, and finally the form is the design form that informs the user what design requirements have been meet.

\subsubsection{Loading Scenarios}

The Loading Scenario Form is required for all design paths. The purpose of the Loading Scenario Form is to determine the required capacity of the member according to $\mathrm{ACl}$ guidelines. Unlike the previous forms, the Loading Scenario Form requires user inputs. There are a total of ten loading conditions that the form requests. The inputs, as seen in Figure 3.3, are the dead load, live load, lateral fluid pressure load, snow load, rain load, wind load, earthquake load, temperature/creep/shrinkage load, soil load, and live roof load. 


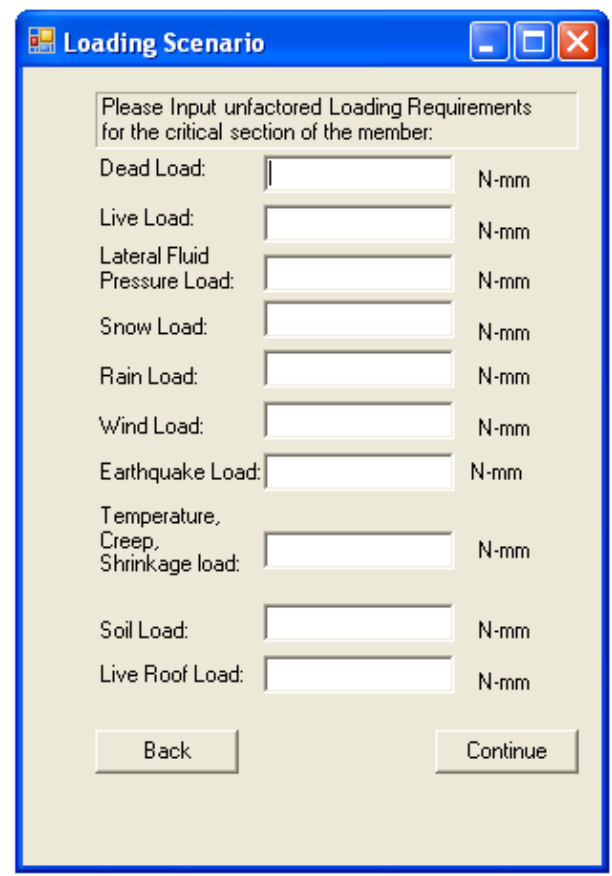

Figure 3.3 Loading Scenario Form

The loading conditions should not be factored for the application will use the inputs to determine the required capacity by utilizing $\mathrm{ACl}^{\prime}$ s loading combinations where the factors are applied. It will be up to the user's prerogative to determine which loading conditions are applicable. For conditions that are not applicable, enter zero.

The inputs must match the units displayed to the right of the input box. As seen in Figure 3.3, for beams in flexure the units are Newton millimeter. For shear strengthening and columns the units are Newton (not shown). If the values given are not in the correct units the application will continue with the assumption that the loading is in the required units which will produce wrong results.

After all loading conditions are given, the user needs to click the "continue" button to proceed in the design process. If one of the conditions does not have a valid number, the application will inform the user that a number was not entered and will focus on the errounous entry. This means that if a 
condition is not being considered, a zero must be placed in the textbox or the application will warn the user as shown in Figure 3.4.

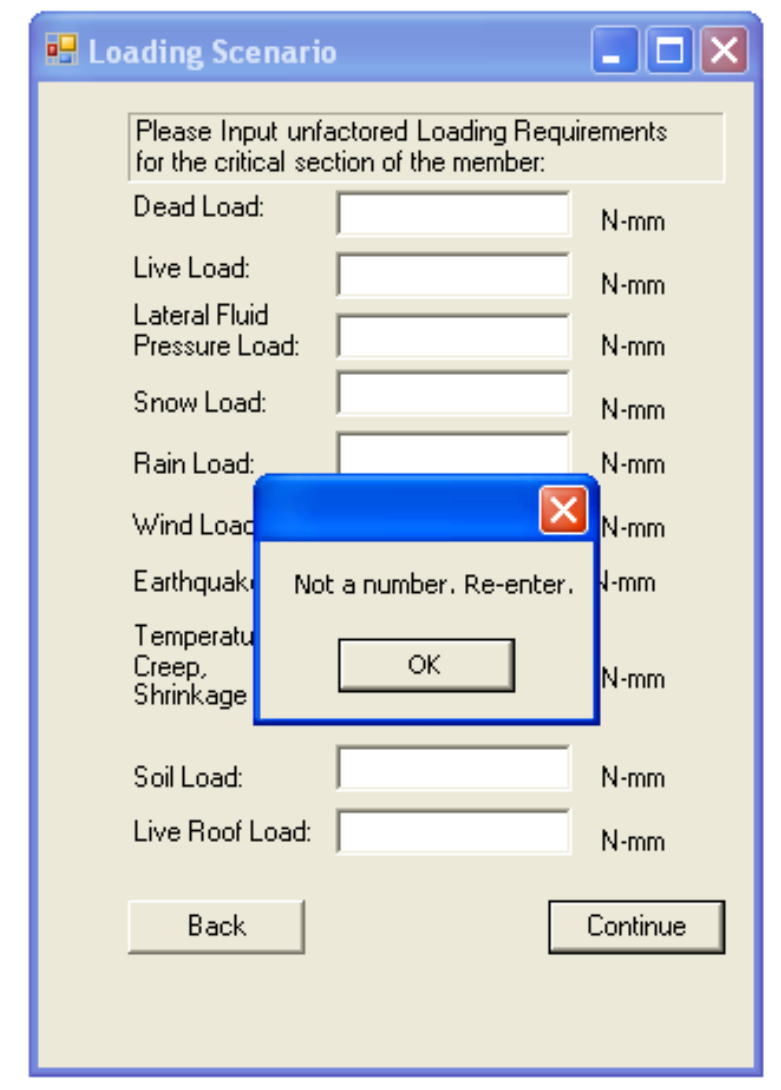

\section{Figure 3.4 Error message for invalid numerical input}

The "back" button will send the user back to the Main Form to start the process over. The application will not store any input values if the user decides to restart the design. 


\subsection{Beams Flexure Properties}

The next form in the strengthening of beams in flexure path is the Beam Flexure Properties

form. The purpose of this form is to gather all the required property values need to continue the design process. The form is broken into five group boxes which each having a unique theme as shown in Figure 3.5. The first group box located in the top left and entitled "Geometric Properties" has four inputs that are required: length of beam, base of beam, height of beam, and the moment of inertia before cracking occurs. The units are once again located to the right of the textbox and the input needs to match them.

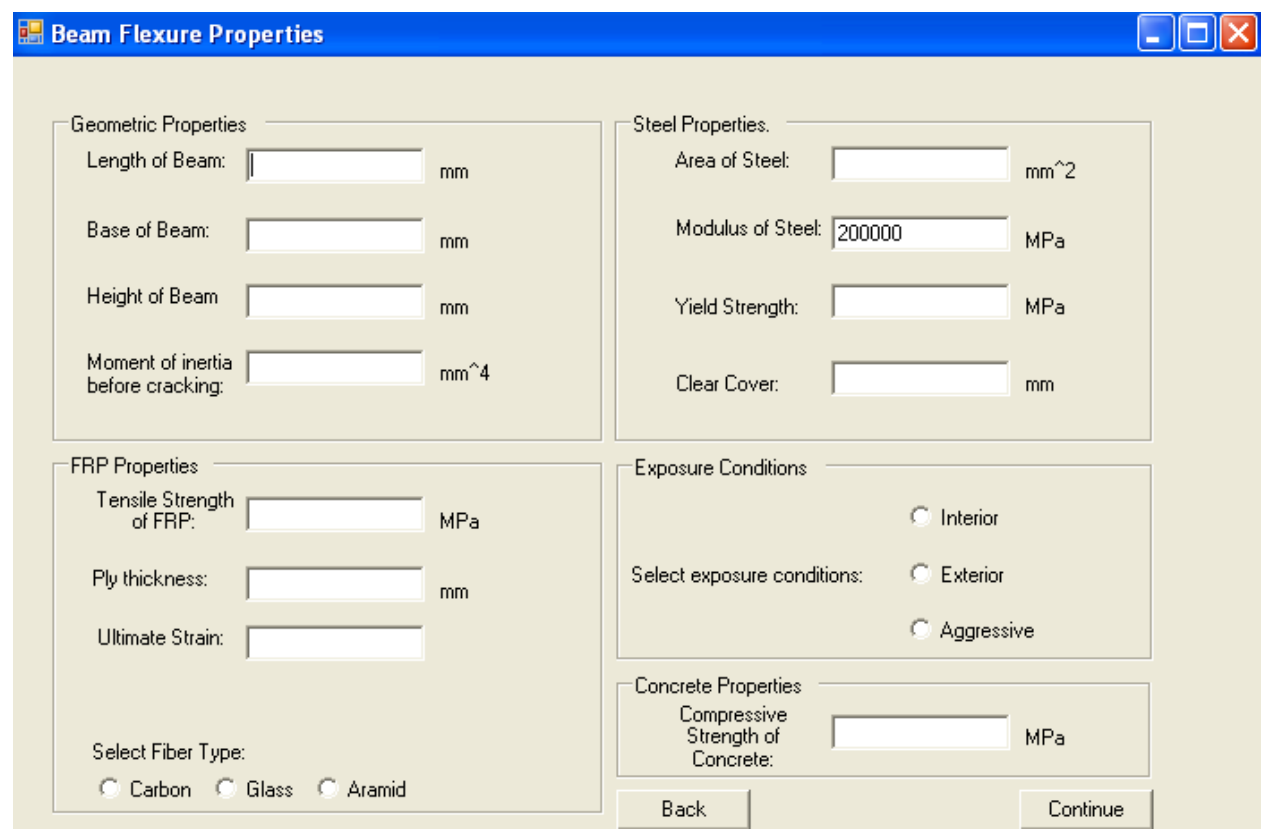

\section{Figure 3.5 Beam Flexure Properties Form}

The group box located in the top right is for all steel properties needed for the design. The inputs are area of steel, modulus of steel, yield strength of the steel, and the clear cover of the tensile reinforcement from the edge of the concrete beam. When the form is initially opened, the modulus of steel textbox is already filled with the value $200000 \mathrm{MPa}$. This is the standard yield modulus of steel but if the user needs to change this value for whatever reason, the textbox is still enabled for the user to edit the textbox. 
The lower left corner is dedicated to all properties related to the FRP. The user will find textboxes for the tensile strength of the FRP, the ply thickness, and the ultimate strain of the FRP. The user will also encounter three radio buttons. The radio buttons provides three options for fiber type: carbon, glass, and aramid. The fiber type is required in order to determine the environmental reduction factor. The application will only allow the user to select one of the three options, and if the user decides to continue the process without selecting one of the three options, the application will warn the user through a popup window that a fiber type was not selected after which the application will select carbon automatically but will still give the user an opportunity to select glass or aramid.

The group box entitled Exposure Conditions located in the right middle of the window form only has three radio buttons: interior, exterior, and aggressive. The radio buttons represent the three type of environmental exposure the beam can experience according to $\mathrm{ACl}$ guidelines. The exposure condition is also needed to determine the environmental reduction factor for the FRP. Just like the fiber type, the application will not allow the user to select more than one radio button, and if the user continues without selecting a condition, the application will once again use a popup box to warn the user that an exposure condition was not selected.

The final group box is for concrete properties. It is located in the bottom right corner. There is only a single textbox which is for the compressive strength of the concrete.

The "continue" and "back" button operates in the same manner as any other form. The "continue" button will progress in the design process after checking to make sure all inputs are valid, and the "back" button returns the user to the Main Form. 


\subsection{Beams Flexure Design From}

The final form for the strengthening of beams in flexure is the Design Form. The form has several facets. The form has a three stage procedure which is initiated by the user clicking "Calculate Results" button located in the top middle of the form as seen in Figure 3.6. Once the button has been clicked, the first stage, Initial Checks, is processed by the application and the results are shown in the group box entitled "Initial Checks."

First the form will make two checks that are required before the FRP strengthening design can be started. The first item checks to see if the original concrete beam has the capacity to meet ACl's minimum loading requirements. The application displays these values to the user in the first two textboxes which both have a light blue background in this group box. The soft yellow textbox right below the minimum loading capacity and the original loading capacity is where the application will inform the user if beam has meant the minimum loading capacity. If the beam has satisfied the requirement the checkbox immediately to the right of the soft yellow textbox will be checked which can be seen in Figure 3.7. This checkbox gives the user an easy verification.

The second initial check that is required is the maximum allowable strengthening. $\mathrm{ACl}$ limits the amount of additional strength that can be added to the original beam. The application will display the maximum allowable strengthening and the actual strengthening that will occur. Both calculations are dependent on initial loading conditions. The soft yellow textbox below the allowable strengthening values will inform the user if the beam meets $\mathrm{ACl}$ maximum strengthening requirements, and the checkbox will be checked if the requirement was met. 


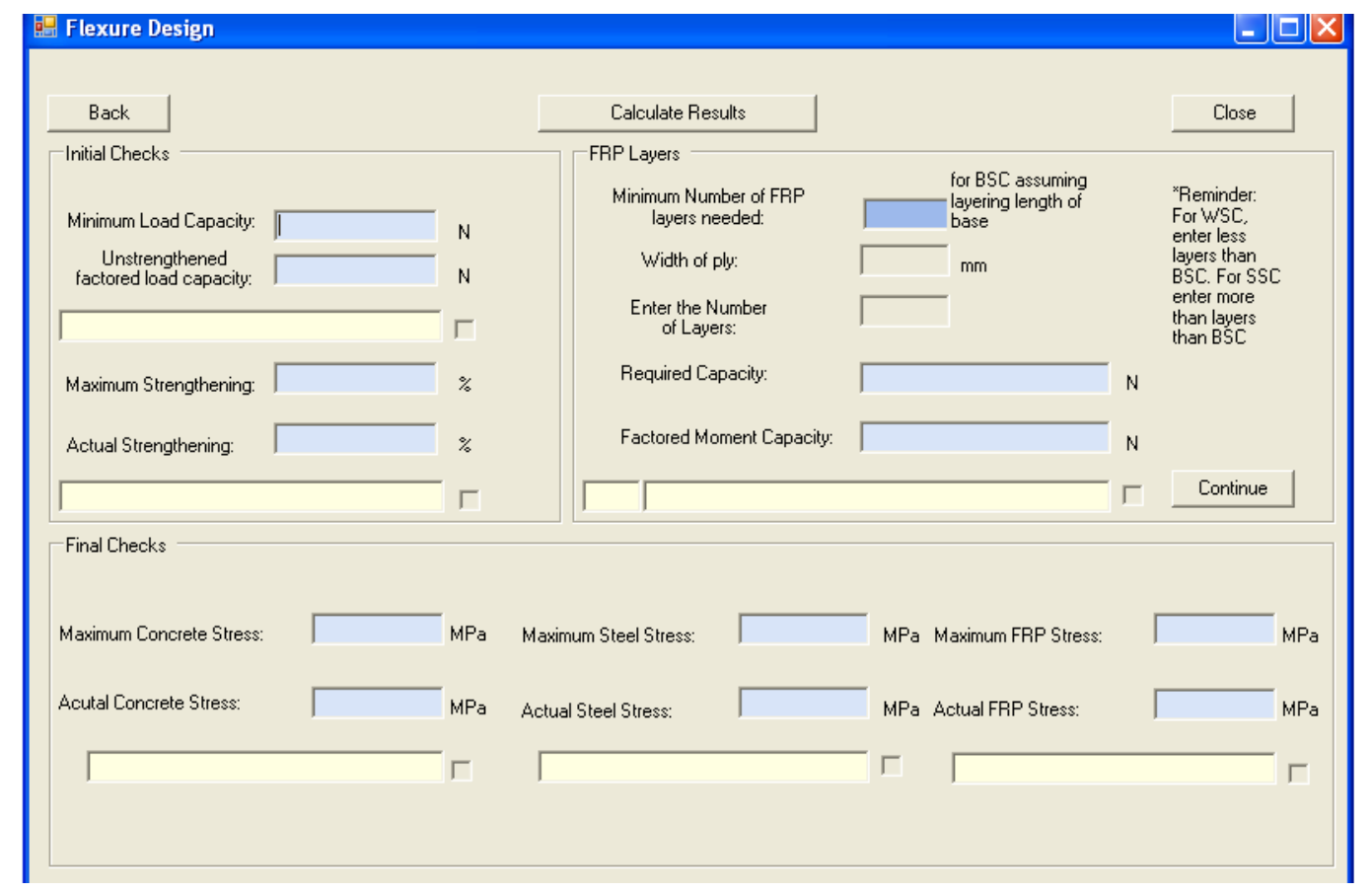

Figure 3.6 Beams Flexure Design Form

It is important to note that none of the textboxes in "Initial Checks" group box are enabled. The textboxes are for read-only purposes; therefore the application prevents the user from having the ability to edit the displayed values.

The user will also notice that in the right group box, "FRP Layers," that a minimum number of layers have an output as well as "Width of ply" and "Enter number of layers" textboxes have become enabled. The application has at this point determined the number of layers needed to meet what is known as the balance strengthening configuration (BSC) while assuming the width of the ply to be the same dimension as the base of the beam. This begins the design stage of the application. 


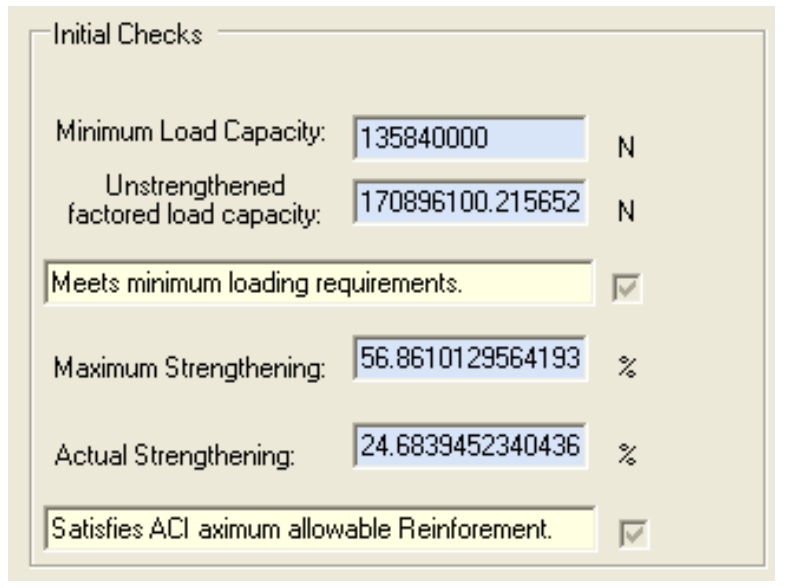

\section{Figure 3.7 Initial Checks Outputs}

To continue the design stage, the user will now need to input the width of the ply and the number of layers the user wishes to use. The group box, "FRP Layers" has a reminder in the upper right corner to inform the user that if a weak strengthening configuration (WSC) is desired, the user must enter fewer layers than the BSC. Otherwise, if the number of layers entered is greater than the BSC, it will be a Strong Strengthening Configuration (SSC). Once the user has provided the width of the ply, which has to be less than or equal to the base of the beam, and the desired number of layers, the user needs to click the "continue" button to finish the design stage and proceed to the last stage.

The "continue" button does check the inputs in the "FRP Layers" group box. If the width of the ply is indeed greater than the base of the beam, a popup box informs the user it is greater than the base. The number of layers is also checked to make sure it is an integer, and if not the application informs the user in the same manner as all other invalid inputs, through a popup box.

The final stage of the form is where the last of the requirements of the design are calculated and checked against standards set by $\mathrm{ACl}$. After the "Continue" button has been clicked, the form will populate the rest of the disabled light blue and soft yellow textboxes, and the check boxes will be checked if the requirement has been meet. In the "FRP Layers" group box, the user will find the required 
capacity, this is where the maximum loading combination that was discussed in the Loading Scenarios form, and the factored moment capacity of the beam after it is strengthened using FRP. The application will display if the capacity is greater than the required amount. Also the smaller soft yellow textbox will display the configuration that is being used. This is not always what the user might expect since the configuration is based on the area of FRP and not just the number of layers.

The group box, "Final Checks," the application displays values for the maximum allowable stress in the concrete, steel, and the FRP. Below those values are the actual stress in the concrete, steel, and FRP. The soft yellow textboxes display if the requirement is meet.

If the user has gotten to this point of the process, the application will show a popup box and inform the user the outcome of the design by either saying the design has meet all requirements or by informing the user that the system needs to be redesigned as shown in Figure 3.8.

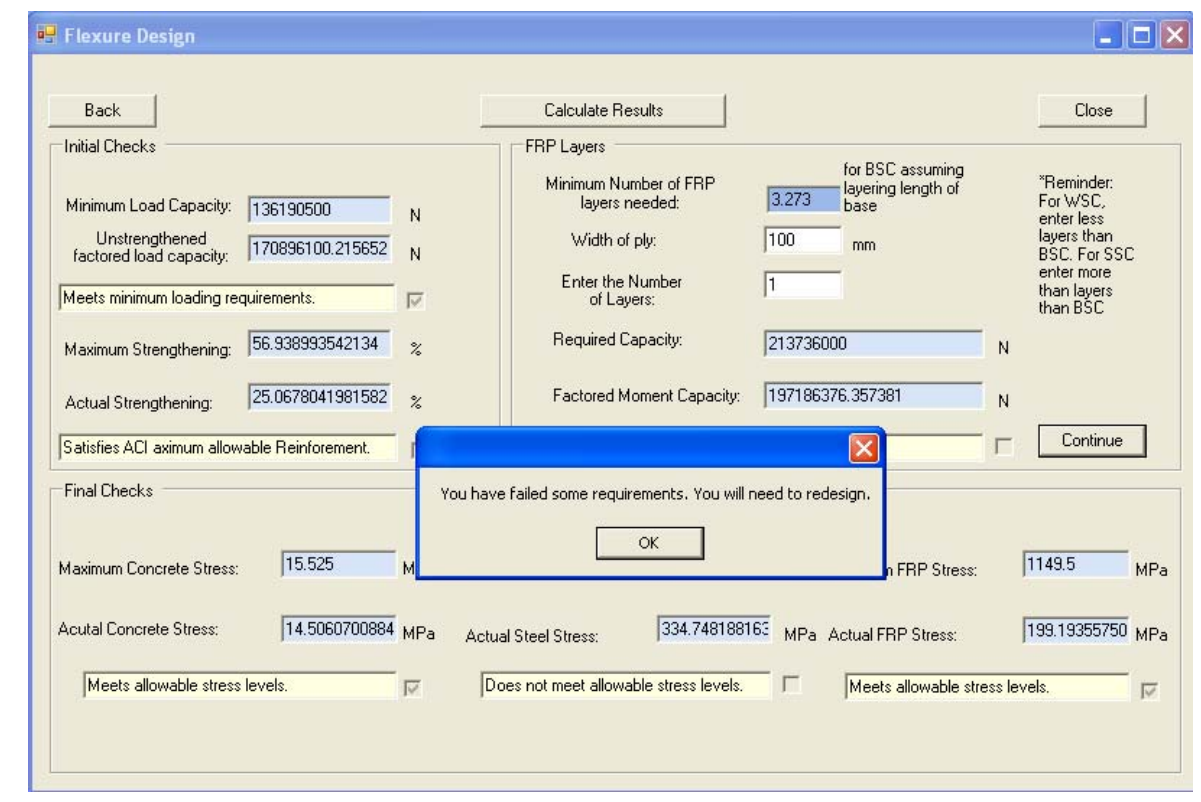

Figure 3.8 Redesign Popup Box

The "back" button returns the user to the Main Form. The "close" button closes the windows form application. 


\subsubsection{Strengthening Beams in Shear}

If the user clicks the button "Shear Strengthening" in the Beams Form (Figure 3.2), the application will continue down the shear strengthening path. This path follows a similar stream as strengthening beams in flexure (3.2.2.1). The path will lead the user to the Loading Scenarios Form where the user must input the loading conditions for the beam. Unlike beams in flexure, the units for this form are Newton, which are displayed to the right of the textboxes. For further explanation of the Loading Scenario form please refer to 3.2.2.1.1 of this chapter.

\subsubsection{Beam Shear Properties Form}

The following form in the Shear Strengthening path is the Beam Shear Properties From. The objective of this form is to retrieve all the required property values need to complete the design process. Similarly to the Beam Flexure Properties From, there are five group boxes. There is only one difference between the Beam Shear Properties Form and the Beam Flexure Properties From. Located in the steel properties is an additional textbox for the spacing of shear reinforcement along the beam as seen in Figure 3.9. All steel properties in this form are related to the shear reinforcement of the concrete beam. When the user clicks "continue" a popup box appears to inform the user that the user must select FRP wrapping options before calculating results. This is relevant to the final form of the path. 


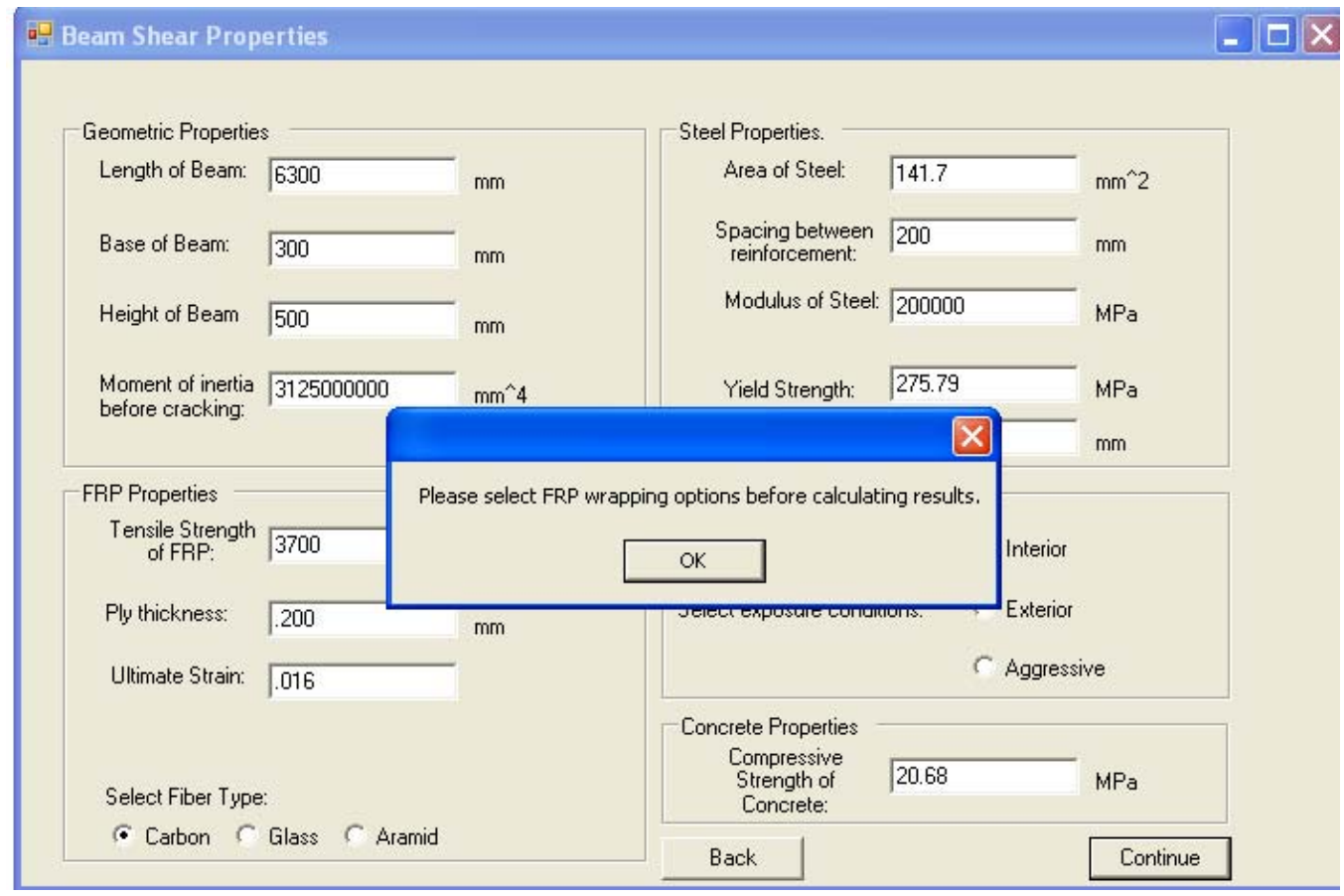

Figure 3.9 Beam Shear Properties Form

\subsection{Beams Shear Design Form}

The final form for the shear strengthening path is the Shear Design Form. Just like the Flexure Beams Design Form, there are three stages: initial checks, design, and final checks. Unlike Beam Flexure Design, to initialize the first stage requires more than just clicking the "Calculate Results" button. Recall the popup box shown before closing the Beam Shear Properties Form that inform the users to select wrapping options before calculating results. The user must do this or the application will inform them again that they must select a style of wrapping.

Three are two small group boxes located inside the "FRP Layers" group box. Each subgroup box represents a parameter of wrapping with different options, which can be seen in Figure 3.10. The left subgroup box requests the user to choose a style of wrapping and presents three options. The complete wrapping option is for when the beam will be wrapped on all sides and assumes the depth of the layer to be the height of the beam which is will be displayed in the depth of wrapping textbox and assumes 
the base of the layer to be the base of the beam. The 3-sided wrapping scheme includes all sides of the beam expect the top. Therefore the application assumes the base of the ply to be the same length as the base of the beam. The last option in this subgroup is 2-sided wrapping. 2-sided wrapping only applies FRP to the sides of the beam. If either 3-sided or 2-sided are selected the form enables the depth of wrapping textbox. The user must input this before clicking "Calculate Results" and it must be less than or equal to the height of the beam.

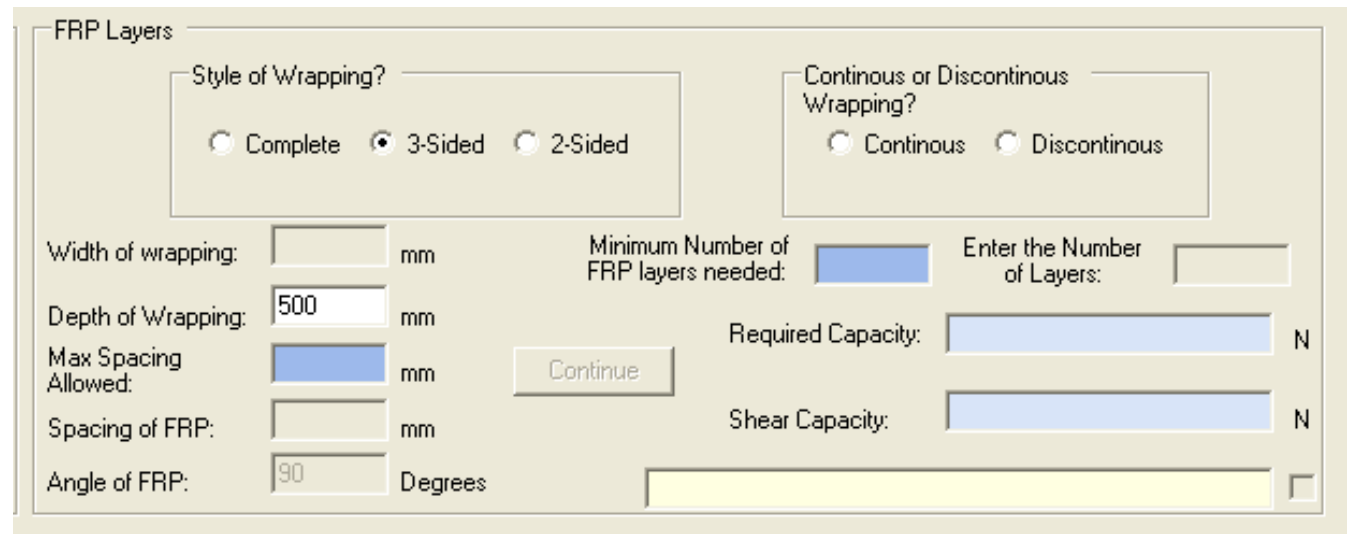

\section{Figure 3.10 Shear Strengthening Wrapping Options}

The second subgroup box located on the right side wants the user to select if the wrapping should be continuous or discontinuous wrapping along the beam. By selecting continuous, the application will assume the whole beam will be wrapped and the angle the FRP is applied to be 90 degrees. If discontinuous wrapping is chosen, the user wants to space the FRP shear reinforcement along the length of the beam. Also by selecting the radio button for discontinuous, textboxes for width of wrapping, and angle of FRP become enabled and must be entered before continuing into the second stage of the form.

Once wrapping options have been selected and the appropriate inputs are given, the initial stage can be initiated. The initial stage is exactly the same as Flexure Beams Results form. The application will calculate and check the minimum load capacity as well as the maximum allowable 
strengthening allowed by $\mathrm{ACl}$. Then the form will inform the user if the original beam meets these requirements. The minimum number of FRP layers that is needed will be displayed in the "FRP Layers" group box. This minimum is for meeting the required shear capacity only. This minimum might not meet other requirements. Therefore, the user must input the number of layers before continuing to the design stage of the form.

Once an integer is given for the number of layers, the "Continue" button will become enabled. If the user has selected discontinuous wrapping, the form will also display the maximum spacing allowed and enable the spacing of FRP textbox. The user must input the desired spacing that is less than the maximum allowable or the application will inform you that the spacing has exceed the maximum. After all these items are attended, the user will need to click the "Continue" button to proceed to the final stage.

During the final stage the application will calculate the required capacity given from the loading conditions and the capacity of the beam after strengthening. It will then inform the user if the design meets the required loading capacity. Unlike Beam Flexure Design Form, the Beam Shear Design Form only needs to check the maximum allowable shear reinforcement, and just like with all other checks, the application checks what is allowable versus what is given and informs the user if the design meets the requirement. Also, a popup box will inform the user if the design meets all requirements or not as seen in Figure 3.11. 


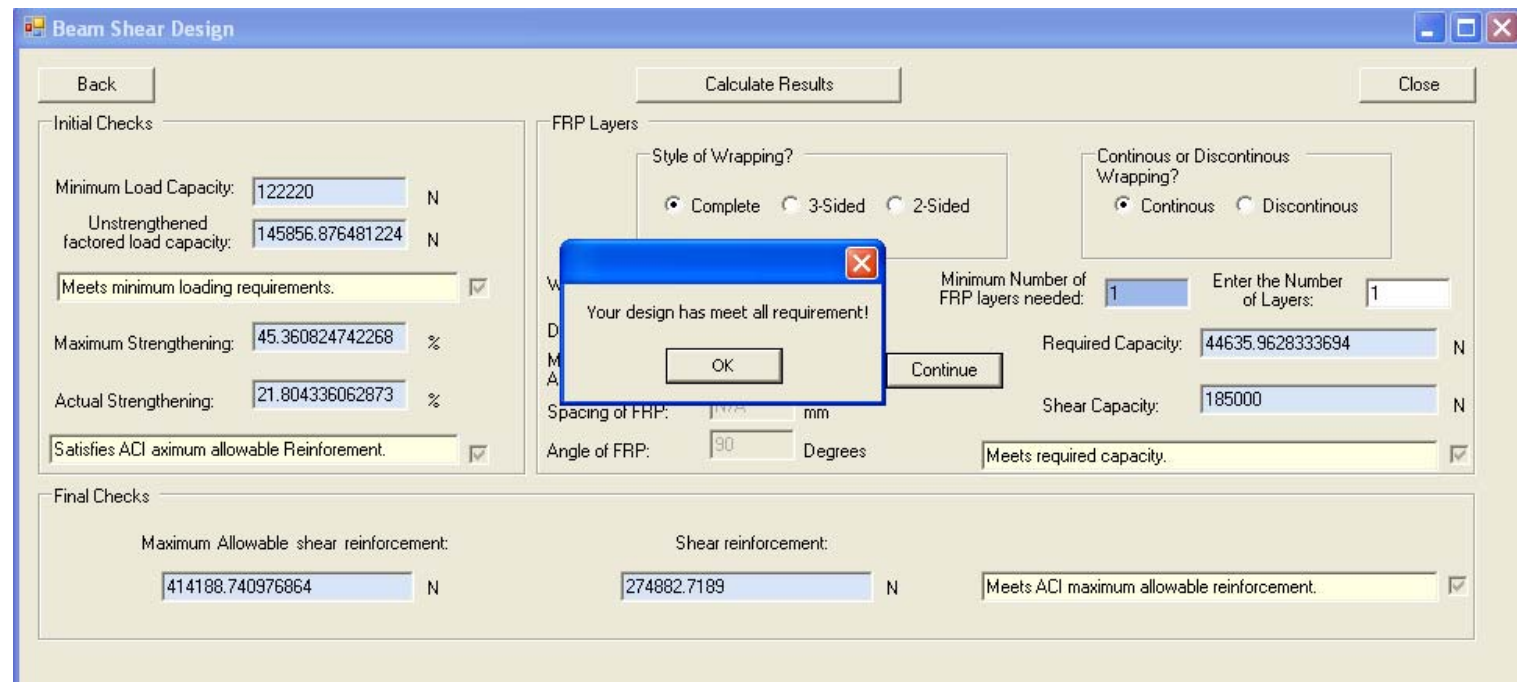

Figure 3.11 Beam Shear Design Form

\subsubsection{Strengthening of Column in Pure Axial Compression}

From the Main Form (Figure 3.1), the user can select Column. The column path is designated for strengthening columns that are only experiencing axial compression. The path has a similar procedure as beams. The user will be guided through the Loading Scenarios Form, a Properties Form, and finally a Design Form. The Loading Scenarios form is identical to the shear path Loading Scenarios. For further reading on the Loading Scenarios form, refer to section 3.2.2.1.1.

\subsubsection{Column Properties}

The objective of the Column Properties From is to input all the required properties for the strengthening of reinforced concrete columns. As with the previous properties forms, the Column Properties Form has five group boxes designated towards the same specific categories. Though, the geometric properties and steel properties do have different textboxes plus the addition of radio buttons as seen in Figure 3.12.

In the geometric properties group box, the user will find several new features compared to beams. The first is that there is only one enabled textbox, the length of the column. The rest of the text 
boxes are disabled when the form is initialed open. Next are two radio buttons: circular and rectangular. These radio buttons are options for the shape of the column. The user will need to select an option. By choosing circular, the diameter textbox becomes enabled and the base of column and height of column become zero. At first the gross cross-sectional area will also equal zero but this is because the diameter textbox is empty. Once the user inputs a diameter, the gross cross-sectional area automatically updates. If the user decides to select rectangular as the shape of the column, the diameter textbox is disabled and the base of column and height of column becomes enabled. Once the user inputs the base and height of the column, the gross-cross sectional area will update itself.

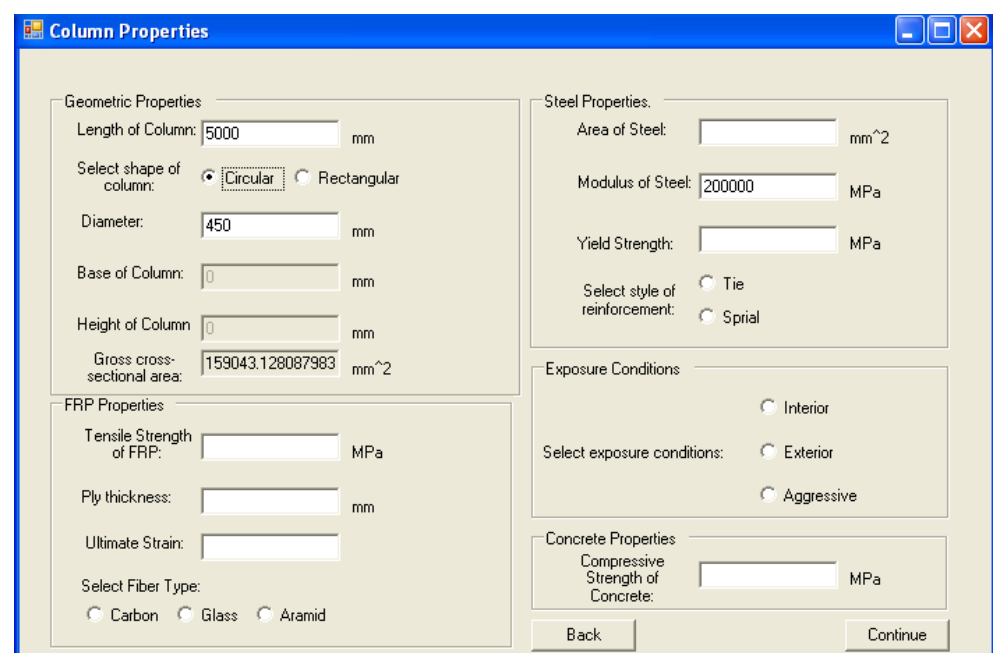

\section{Figure 3.12 Column Properties Form}

The steel properties group box has less textboxes compared to beams, but there is the addition of radio buttons. These radio buttons give options to what reinforcement scheme will be used. The user has the option of tie style reinforcement or spiral style reinforcement. A scheme has to be selected or the application will not advance to the next form.

The FRP properties, exposure conditions, and concrete properties group boxes are identical to beams properties. Please refer to section 3.2.2.1.2 Beam Flexure Properties for further explanation on 
the group boxes. All values must be numerical or the application will use a popup box to inform the user of the conflict and direct to the appropriate text box.

\subsubsection{Column Design Form}

The final form of the strengthening of columns is the Column Design Form. This form, as the other results forms, has three stages. The initial stage begins by the user clicking the "Calculate Results" button, and will produce the same checks as beams. For more reading on the initial checks, please read section 3.2.2.1.3 Beam Flexure Design.

After the initial stage is completed, the form informs the user about the minimum number of layers needed. The number of layers textbox is enabled, allowing users to input how many layers to strengthen the column. This begins the design stage of columns. The design stage of columns is the easiest of the strengthening paths implemented. There is no need for schemes, or configurations, as there were in shear strengthening and flexure strengthening. The user only needs to input an integer for number of layers and click the "Continue" button which is also enabled as seen in Figure 3.13.

The final stage of the Column Design Form consists of four checks. Located in the top left corner of the final checks group box is the check for minimum level of confinement. $\mathrm{ACl}$ has a predetermined minimum level of confinement of 0.08 which is displayed in the read-only textbox. The maximum compressive strain is also a predetermined number set by $\mathrm{ACl}$. The maximum compressive strain of 0.01 is also being displayed in the read-only textbox. These checks plus maximum concrete stress and maximum steel stress all follow the same pattern. The application will compare the required or limiting value to the value of the strengthened concrete column and inform the user if the column has met each requirement. 


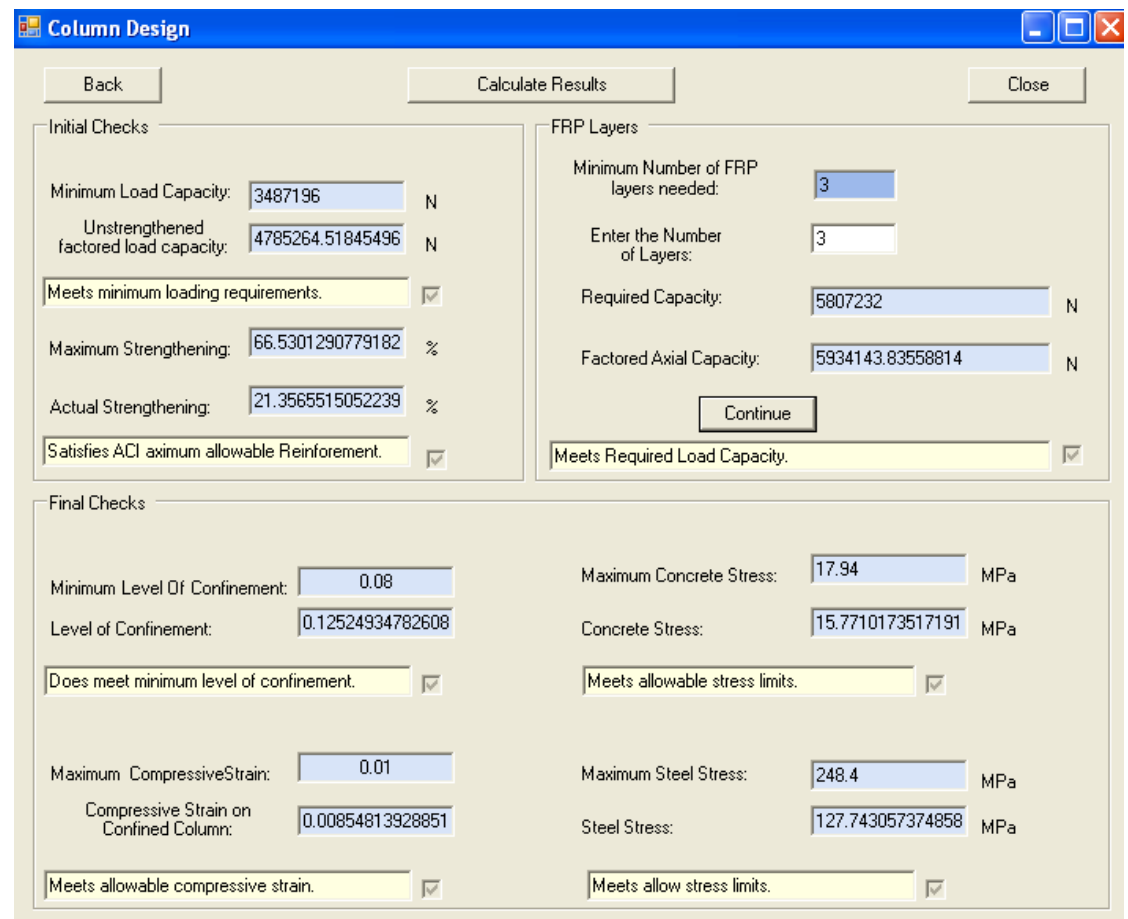

Figure 3.13 Column Design Form

\subsubsection{Beam-Column Verification}

The third and final option available to the user on the Main Form is Beam-Columns (Figure 3.1).

The strengthening of beam-columns follows a completely different path because it is not a design

procedure as beams and columns are. The Beam-Columns path is used for verification of the

strengthened design. Therefore, instead of the application informing the user of how many layers of FRP

is required and analyzing the layers the user inputs, the beam-column path will just do that analysis and display the interaction diagram that will allow the user to use to check the design.

The Loading Scenarios Form is not used. The user will have to provide the factored loading combinations following $\mathrm{ACl}$ guidelines for the required moment and axial compression capacity of the member. 


\subsubsection{Beam-Column Properties}

Just as the paths for beams and columns required the user to provide required properties for the procedure, so does beam-columns. The Beam-Column Properties Form has the same five group boxes that can be found on beams and column plus an additional group box. Of the five original group boxes only the exposure conditions group box is identical to all other property forms. This is evident in Figure 3.14. Also, the geometric properties group box is identical to the geometric properties group box that is used in columns. Please refer to section 3.2.3.1 to read more about the geometric properties.

The FRP properties group box has the same items as beams and columns with the addition of a textbox for number of layers. Recall the strengthening of beam-columns path is not a design path but rather a verification path. Therefore, the application needs to know how many layers are applied in the strengthening of the beam-column. The user can use the verification process as an iterative design procedure to determine how many layers meet the requirements.

The concrete properties group box only has one item, compressive strength of the concrete. Previously the user could input in a textbox any value. The beam-columns path does not allow this. Instead there is a drop-down menu for the user to select the available concrete compressive strengths. By clicking the down arrow, the user will see three options: $20.68,27.57,34.47$. The reasoning for allowing the user only these options is because the application must determine the appropriate confined stress block parameters, which depends on the concrete compressive strength, and the application only have such parameters for the three available compressive strengths. 


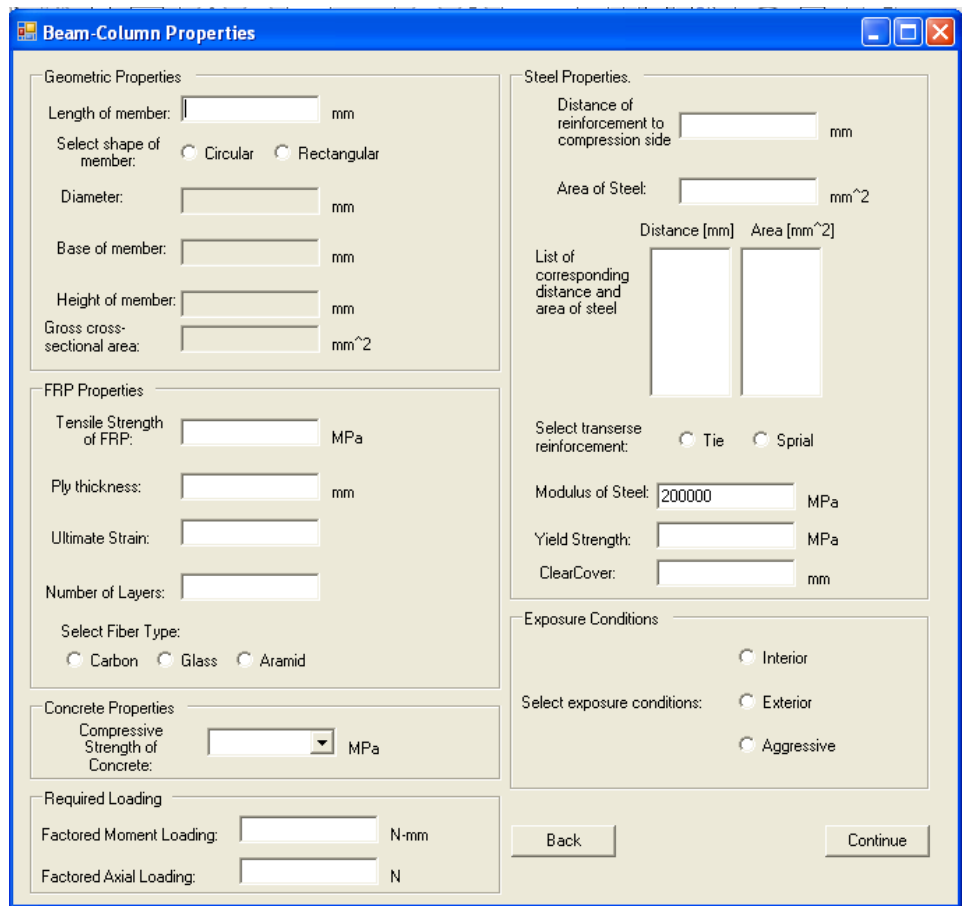

\section{Figure 3.14 Beam-Column Properties Form}

The group box below the concrete properties group box is for the required loading. As stated previously, this is a verification procedure. Therefore, in order to verify the strengthen design; a loading requirement is needed to be checked against. There are two textboxes located within the group box. The first one is for the factored moment loading, and the second is for the factored axial loading. Both loadings need to be factored by $\mathrm{ACl}$ guidelines for loading combinations.

The most significant change among the Beam-Column Properties Form compared to other properties forms is the steel properties group box. Because the verification process, the application will need to know more detail about the steel reinforcement. It will need to know how far each layer of reinforcement is to the compressive side of the member and how much area each layer has. A closer examination of the layout of this group box is needed to assist in the explanation for what the user is required to input and how to input these said requirements. Figure 3.15 will show two textboxes and two list boxes. The list boxes will show the corresponding inputs the user gave for a single layer of 


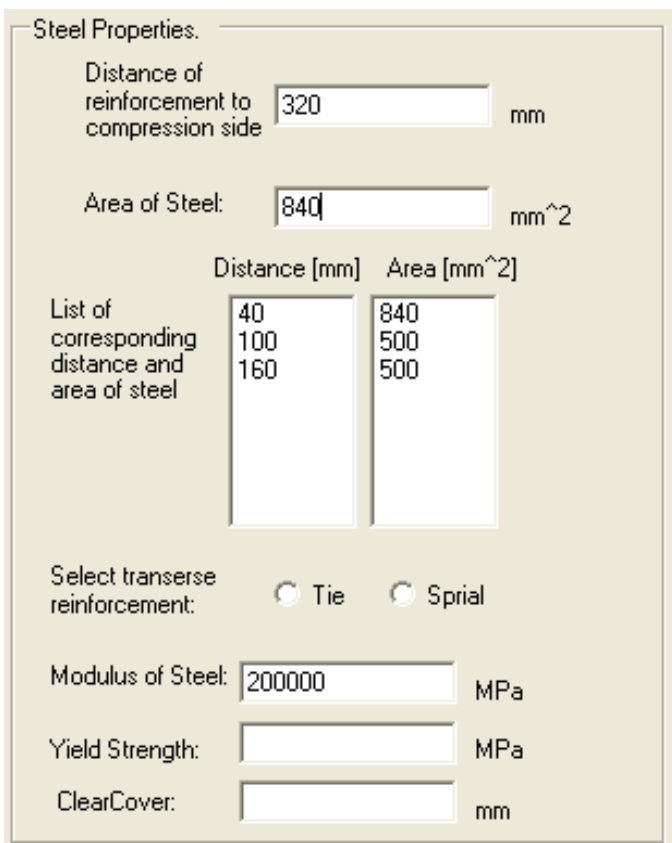

(a)

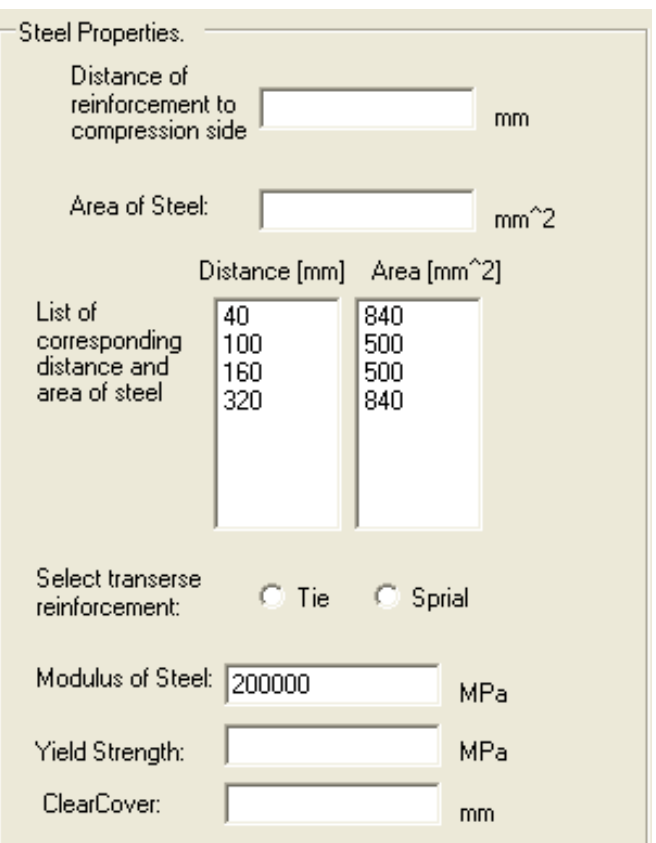

(b)

\section{Figure 3.15 Steel Reinforcement Inputs for Beam-Columns}

reinforcement. So each time the user inputs a distance and an area (Figure 3.15a), the list boxes will automatically update with that value, and the textboxes are clear and ready for another layer (Figure 3.15b). The user must input all the steel reinforcement layers. Do not input more than one distance and area in at a time. The application will not distinguish the difference between the multiple inputs. If the layer's distance and area show up in the appropriate list boxes parallel with each other, the application will associate that distance with that area.

\subsubsection{Interaction Diagram}

The application will produce an interaction diagram to verify the design as illustrated in

Figure3.16. The interaction diagram displays the nominal capacity at failure modes of the beam column with respect to stress resultants, the axial force, and bending moment. When the interaction diagram is initially opened, the form is blank except for the legend and three buttons located on the top of the form. The "Back" button returns the user to the Main Form, and the "Close" button closes the form. 
The "Show Results" button when clicked will produce the interaction diagram. The diagram will produce four lines and a single point. The single point is a blue rectangle which displays the required loading that was given by the user in the Beam-Column Properties Form. The yellow line displays the nominal strengthened capacity. The dark green line represents the nominal capacity of the original beam-column. The red line represents the factored strengthened capacity, and the grey line is the factored original capacity. Figure 3.16 demonstrates a typical interaction diagram.

If the blue dot, the required factored loading, is below the red line, factored and strengthened beam-column, the design is adaquete. Else, the strengthening scheme needs to be redesign and requires producing a new interaction diagram.

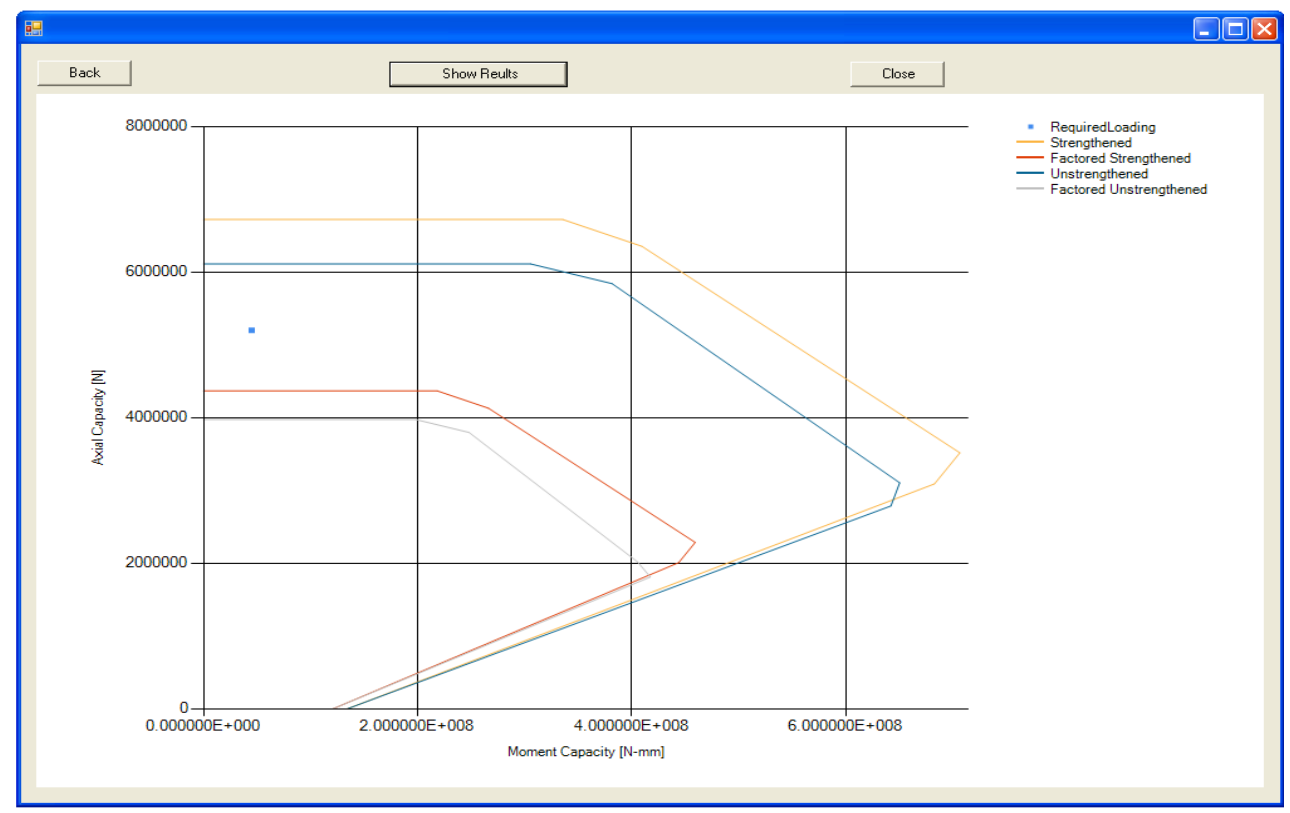

Figure 3.16 Interaction Diagram 


\section{Chapter 4 Software Methods}

\subsection{Introduction}

\subsubsection{Design Overview}

The software methods for the design process for strengthening reinforced concrete beams and columns was chosen to be programmed in the C\# language because of the common use of $\mathrm{C \#}$ in the workforce and because it is object oriented allowing the classes, a template to classify or describe an object, and methods, actions an object can perform, to be used by other programmers who wish to use these methods and process with much greater ease and quickness. Instead of an individual needing to recreate the methods, the programmer can create an object class using the classes described in this chapter.

The structure of the application was designed to make the process very linear for the user, not allowing the user to fall away from the designed path for the given concrete structure. Though, the software method is meant to streamline the design process and allow others a set of classes to use in developing a different design procedure, the application does hinder the design process because of the linear path used. Further development may address this.

More importantly, the software method was constructed so that if a different design procedure were to be employed, the classes are still useable because the classes do not conduct any major decision. For this application, all design processes are implemented in the event handlers which execute the design steps. This chapter discusses the structure of the windows application (App) and each individual class and windows form used in the application. 


\subsubsection{System Architecture}

The system architecture is displayed in Figure 4.2 and demonstrates the flow of the application.

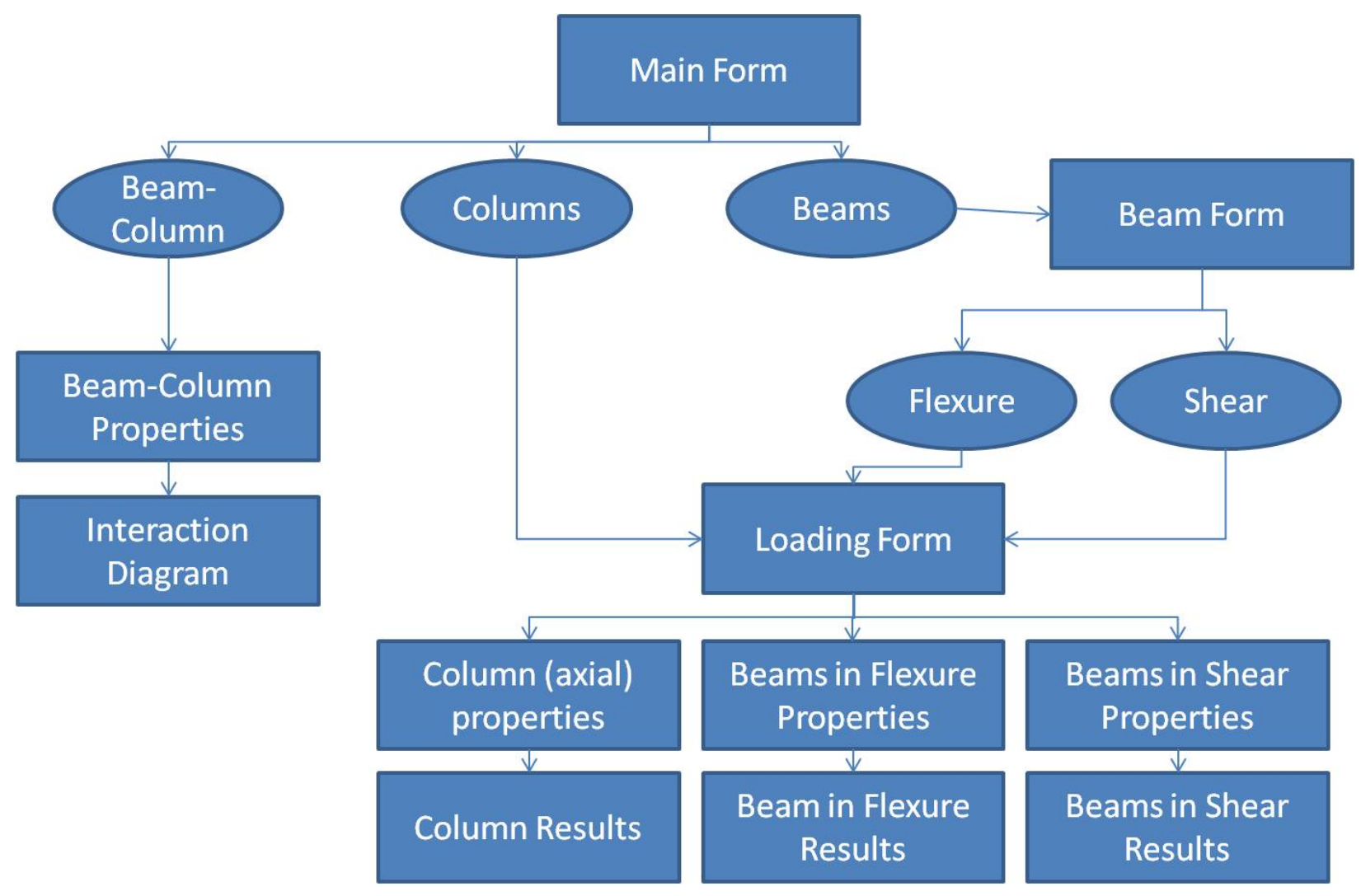

Figure 4.1: System Architecture

The application has four potential paths, one for each type of strengthening and one for beamcolumn verification. Each path requires a properties form for inputting required properties of the structure needed to complete the design and verification. The three strengthening paths will have a results form displaying the outcomes while the beam-column will instead have a form for displaying the interaction diagram

\subsection{System Components}

The system components are broken down into three major categories: classes, forms, and resource files. Classes are templates used to create "objects" which can be created within any windows 
application. Objects that are created from a certain class can use the class methods defined within that class. In this case, each class represents a significant step or procedure in a design process, with each class method implementing the required calculations needed for a particular value to be used again in the design process.

The forms are used as the medium for the user to interact with the application. The event handlers are used to execute and guide the design process. The forms are where objects are created and used within the design process.

The resource file is used to store strings that are used on a frequent base. For this application, it contains the upper and lower limits for validating any input within the application.

This chapter explains classes and forms with, each class broken down into individual methods where each class method purpose and procedure is discussed. Each form purpose and important features are discussed with individual event handlers, which process an action done after a user has interacted with the form. They are further broken down into specific purposes and processes.

\subsection{1 "clsLoading" class}

The class "clsLoading" is the first class to be used in the three design paths of the application. The purpose of the "clsLoading" class is to determine the required strength of the member dependent on the loading combinations given by Eq. 2.29 through Eq. 2.35.

Since the objective of the class has one singular purpose, there is only one method the class can use. The method "MaximumLoadingScenerio" can be used to return a double value for the maximum required strength. The method calls on all ten varieties of loads needed to the loading combinations (Eq. 2.23-2.35). These loads must all be double float values. 
When the method is used, 17 different possible loading combinations are calculated. All these combinations are provided by $\mathrm{ACl}$. Once the combinations are calculated, each one is put into an array. That array will then use a built-in array class method called max. The max method returns the largest value in the array. This value is the maximum loading combination and therefore the maximum required strength. The method will return the max required strength as double float value.

\subsection{2 "clsFRPEnvironmentalFactor" class}

$\mathrm{ACl}$ 440.2R-08 uses an environmental strength reduction factor for FRP systems and exposure conditions. Class "clsFRPEnviornmentalFactor" is a single method class for determining the reduction factor based on two parameters: exposure conditions and fiber type. The class is used for all program paths including beam-columns.

The method, "EnvironmentalFactor", only requires two integer inputs, fiber type and exposure, which represents different cases. Therefore a double switch-case is implemented with exposure conditions being considered first. $\mathrm{ACl}$ considers three different exposures that have different reduction factors dependent on fiber type. The method uses 1 as interior conditions, 2 as exterior conditions, 3 as aggressive environments. For fiber types, 1 represents carbon, 2 for glass, and 3 for aramid.

Using the switch-case conditionals, the method will first look for the correct exposure condition followed by fiber type. Each branch of the switch case has a unique value for the environmental reduction factor which is returned as a double float value by the method. For example if the condition is interior and the fiber is glass, the method will go to exposure case 1 , representing the interior exposure condition, and fiber type case 2 , representing glass, to obtain a value of 0.75 for the reduction factor and return that value to the designated variable within the application. 


\subsection{3 "clsFRPSystem" class}

The strengthening system being used must be evaluated for the modulus of the FRP, the ultimate rupture strain, and the ultimate tensile strength. The "clsFRPSystem" class evaluates these values using three methods, one for each FRP property. The class methods are extremely short because of the simplicity of calculations required to produce the properties.

The first class method is "UltimateTensileStrengthOfFRP" which main purpose is to calculate the ultimate tensile strength of the selected FRP system. The environmental factor and the tensile strength of the FRP are required double float values for the method that are multiplied (Eq. 2.2) to determine the ultimate tensile strength which is returned by the method as a double float value.

The "UltimateRuptureStrainOfFRP" uses the environmental reduction factor and the ultimate strain of FRP to produce the ultimate rupture strain of the FRP (Eq. 2.3). The method then returns the rupture strain to the application as a double float value.

The last of these short methods is the "ModulusOfFRP" method. The method class for the ultimate rupture strain of the FRP and the ultimate tensile strength of the FRP both of which are double float values. Though these values do not have the calculated values from the previous "clsFRPSystem" methods, they must both be either factored or not factored. The modulus of FRP is calculated by the method by taking the tensile strength and dividing it by the strain (Eq. 2.4). The method will return the value as a double float type.

\subsection{4 "clsOriginalSection" class}

The "clsOrignialSection" class encompasses the initial analysis of the original non-strengthened sections for beams in flexure, beams in shear, and column under axial compression only. The class 
includes these different means of strengthening because of the similarity of the procedure. The only difference is how the member capacity is calculated which is dependent on what is being strengthened.

The class has four main uses surrounding $\mathrm{ACl}$ initial requirements for strengthening. The class calculates the minimum loading requirement, the nominal capacity of the member, the maximum allowable strengthening and the actual amount of strengthening. Each member type will use the same methods for minimum loading requirement, the maximum allowable strengthening and the actual amount of strengthening. Each member has its own method to determine the nominal capacity.

\subsubsection{1 “MinimumLoadCapacityOfUnstrengthendColumn" method}

The minimum loading capacity can be calculated by using the class method "MinimumLoadCapacityOfUnStrengthenedColumn". The name should not mislead the objective of the method. The process is independent of the member type. According to $\mathrm{ACl} 440 . \mathrm{R} 2$, "the existing strength of the structure should be sufficient to resist a level of load as described by Eq. 2.1" Therefore the minimum capacity is only dependent on the dead and live loads applied to the structure which are the only two requirement of the method, both being double float values. The method uses Eq. 9.2 to determine the minimum load capacity and returns it as a double float value to the application.

\subsubsection{2 "UnstrengthenedCapacityOfUnstrengthenedColumn" method}

The next class method to be found in "clsOrignialSection" is the method for determining the capacity of a non-strengthened column called "UnstrengthenedCapacityofUnstrengthenedColumn." The method does require several inputs related to geometric and material properties listed in Table 4.1. The table will also inform what variable type the method input has to be. 


\section{Table 4.1 Inputs for "UnstrengthenedCapacityOfUnstrengthenedColumn"}

\begin{tabular}{|c|c|}
\hline Required Input & Variable Type \\
\hline Reinforcement Style & Integer \\
\hline Concrete Compressive Strength & Double Float \\
\hline Gross Cross-sectional Area & Double Float \\
\hline Area of Steel Reinforcement & Double Float \\
\hline Yield Strength of Steel & Double Float \\
\hline
\end{tabular}

The reinforcement style input is used for the method to distinguish between spiral

reinforcement and tie reinforcement. Each reinforcement style has unique reduction factors which the method takes into account by checking the reinforcement style input. If said input is equal to 1 corresponding to tie steel reinforcement, the method will set the strength reduction factor to 0.65 and the eccentricity factor to 0.80 . When the reinforcement style is equal 2 for spiral reinforcement, the strength reduction value is set to 0.75 and the eccentricity factor to 0.85 .

Once reduction factors are set, the method calculates the nominal compression capacity using Eq. 2.20. The value is returned to the application as a double float type.

\subsubsection{3 “MaxPerecentActual” Method}

The method's purpose to calculate the percent of actual strengthening reinforcement that member will need to meet the required loading. The method requires two inputs of type double: the nominal capacity of the non-strengthened member and the required loading. Taking the percent difference of the factored capacity and minimum required capacity is implemented and the percentage is returned to the application as a double float. 


\subsubsection{5 “MaxPercentAllowable" method}

Just as "MaxPercentActual," the method produces the percent of maximum allowable strengthening of the member by using the percent of the difference of required strength and the minimum required strength. Therefore the "MaxPercentAllowable" method needs the minimum load capacity and the max loading requirement, both of type double float. The maximum percent allowable reinforcement will return as a double float variable.

\subsubsection{6 "StressBlockFactor" method}

To calculate the nominal capacity of a reinforced concrete beam, several parameters must be calculated prior. The "StressBlockFactor" method determines the stress block factor $\beta_{1}$ which is only dependent on the compressive strength of the concrete of type double float. Eq. 2.44 dictates the formulation of $\beta_{1}$ in SI units.

If the concrete compressive strength is equal to or less than $27.57 \mathrm{MPa}, \beta_{1}$ is equal to 0.85 . Else if the compressive strength is greater than $27.57 \mathrm{MPa}$ and less than or equal to $55.15 \mathrm{MPa}$, " $\beta_{1}$ shall be reduced linearly at a rate of 0.05 for each 6.9MPa of strength in excess of $27.57 \mathrm{MPa}$ " ( $\mathrm{ACl} 318-08)$. Though $\beta_{1}$ cannot be taken less than 0.65 which is why the conditional statement the method checks is limited to $55.15 \mathrm{MPa}$. $\beta_{1}$ will be returned as a double float variable.

\subsubsection{7 "NeutralAxis" method}

This method only purpose to calculate the neutral axis of the soffit beam. The method is very straight forward but requires several inputs all of which are double float variables. To calculate the neutral axis of the original beam, the area of steel reinforcement, the steel yield strength, the concrete compressive strength, the stress block factor $\left(\beta_{1}\right)$, and the base of the beam are required. 
When the "NeutralAxis" method is called, the method will use Eq. 2.12 but assuming the area of FRP is equal to zero and that the steel does indeed yield. The neutral axis is returned by the method as a double float variable.

\subsubsection{8 “MomentCapacityOfOrignialSection" method}

The moment capacity of the soffit section can be calculated using this method. Similarly to other methods, it simply computes the capacity by using a single equation and returns it to the application as type double float. The method uses Eq. 2.12, and therefore the method requires the following inputs all of double float variables: area of reinforcing steel, yield strength of steel reinforcement, depth of the tensile steel from the extreme compression end of the beam, the neutral axis of the soffit beam, and the stress block factor $\left(\beta_{1}\right)$.

\subsubsection{9 "ShearCapacityConcrete" method}

The class "clsOrignialSection" not only calculates capacities of columns and beams in flexure, but also determines the shear capacity of a beam. The method "ShearCapacityConcete" calculates the capacity of the concrete for shear strength using Eq 2.61 which needs the base of the beam, the depth of the tensile steel from the extreme compression end of the beam, and the concrete compressive strength. The method returns the shear capacity of the concrete to the application as a double float variable. It must also be noted that a built-in class called "Math" and the "Math" class method "Sqrt" are used. This is because there are no square root operators in C\#.

\subsubsection{0 "ShearCapacitySteel" method}

Just like the shear capacity of steel, the steel shear reinforcement capacity can be calculated using the method "ShearCapacitySteel." The method calculates the steel capacity by using Eq. 2.65. The inputs called by the method are the yield strength of the steel reinforcement, the area of shear steel 
reinforcement, the depth of the tensile steel from the most extreme compression end of the beam, and the spacing of the steel shear reinforcement. All input are double float variables. The capacity of the steel is returned as a double float type to the application.

\subsubsection{0 "ShearFactoredCapacityBeam" method}

The "ShearFactoredCapacityBeam" method determines the shear capacity of a beam. The method reduces the sum of the concrete and steel shear capacities by the strength reduction factor. Therefore, the method inputs are the strength reduction factor, the shear capacity of the concrete, and the shear capacity of the steel. All of which are type double float. The factored shear capacity of the beam will be returned as a double float.

\subsection{5 "clsAxialColumnDesign" class}

The class "clsAxialColumnDesign" includes all methods need to complete the strengthening design of any concrete column experiencing only axial loading. The class has five methods which vary from initial calculations that will be carried through the design process to the number of plies needed and the new capacity of the column after addition reinforcement is applied.

\subsubsection{1 "FRPEfficiencyFactor" method}

$\mathrm{ACl} 440.2 \mathrm{R}-08$ requires a shape factor to be incorporated when determining the confined pressure of the column. The "FRPEfficiencyFactor" method determines what value of that factor should be used depending on the cross-sectional shape of the column by using Eq. 2.23. The method requires several inputs which can be seen in Table 4.2.

The method will use the geometry integer to check the shape of the column. If geometry is a 1 , the FRP efficiency factor will be set to 1 as defined in section 12.1 .1 of $A C l 440.2 R-08$ because the 
column is considered circular. Now, if geometry is equal to 2 , the method will assume the shape to be non-circular and use Eq. 2.23. The factor will be returned as type double float.

\section{Table 4.2 Inputs for "FRPEfficiencyFactor" method}

\begin{tabular}{|c|c|}
\hline Required Input & Variable Type \\
\hline Geometry & Integer \\
\hline Base of Column & Double Float \\
\hline Height Of Column & Double Float \\
\hline Gross Cross-sectional Area & Double Float \\
\hline Area Of Steel & Double Float \\
\hline AeAc & Double Float \\
\hline
\end{tabular}

\subsubsection{2 "AEAC" method}

When the column cross-section is non-circular, the longitudinal steel reinforcement ratio is needed to calculate the FRP efficiency factor. Method "AEAC" will calculate or return a zero value for the reinforcement ratio dependent on the geometry integer input. The required inputs are listed in Table 4.3.

When geometry is 1 , the longitudinal steel reinforcement ratio is zero. The method sets the ratio to zero only because the ratio is required in other methods, for example the "FRPEfficiencyFactor" method which only uses it when it is a non-circular cross-section but the method requires the ratio in its inputs. Therefore it is easier to just use both methods and have those function no matter of the crosssection instead of creating unique methods for different shapes. 


\section{Table 4.3 Inputs for "AEAC" method}

\begin{tabular}{|c|c|}
\hline Required Input & Variable Type \\
\hline Geometry & Integer \\
\hline Base of Column & Double Float \\
\hline Height Of Column & Double Float \\
\hline Gross Cross-sectional Area & Double Float \\
\hline Area Of Steel & Double Float \\
\hline
\end{tabular}

If the geometry is 2 , meaning a non-circular section, the method will set a local double float variable "roundedcorners" to 0.25 . First this is a local variable because it is only needed to calculate the longitudinal steel reinforcement ratio. The rounded corners variable is the radius of the corners of the column which are typically shaved down to a radius of $25 \mathrm{~mm}$ [4]. It was created as a variable to also allow for easy adjustment if the typical radius changes. Also, when the cross-section is non-circular the method uses Eq. 2.25 to calculate the longitudinal steel reinforcement ratio. Once the ratio has been set to zero or calculated, it is returned as a double float type.

\subsubsection{3 "ConfinedPressure" method}

The confined pressure caused by the FRP wrapping of the column can be calculated by using the "ConfinedPressure" method. The required inputs are available in Table 4.4. The method determines the strength reduction factor and the eccentricity factor based on the reinforcement style integer. If the integer is 1 for tie reinforcement, the strength reduction factor is 0.65 and eccentricity factor is 0.80 . Else, if the reinforcement style integer is equal to 2 for spiral reinforcement, the strength reduction factor is 0.75 and the eccentricity factor is 0.85 . 
After the factors are set, the method will use Eq. 2.22 to calculate the pressure produced from confining the concrete column with FRP, and return the value as a double float to the application.

Table 4.4 Inputs for "ConfinedPressure" method

\begin{tabular}{|c|c|}
\hline Required Input & Variable Type \\
\hline Reinforcement Style & Integer \\
\hline Number of Layers & Double Float \\
\hline Modulus of FRP & Double Float \\
\hline Ply Thickness & Double Float \\
\hline Diameter & Double Float \\
\hline Ultimate Rupture Stain of FRP & Double Float \\
\hline
\end{tabular}

\subsubsection{4 "RequiredNumberOfLayers" method}

This method is used to calculate the number of layers that would be needed to meet the confined pressure of the concrete column. The method requires several inputs summarzied in Table 4.5. The method first initiates four local variables: strength reduction factor for the FRP, the compressive strength of the confined column, the confined pressure, and the number of layers. Each local variable will be discussed in greater detail in this section.

First the method will determine the strength reduction factor and the eccentricity factor in the same manner as explained in the "UnstrengthenedCapacityofUnstrengthenedColumn" method and the "ConfinedPressure" method. 
Table 4.5 Inputs for "RequiredNumberOfLayers" method

\begin{tabular}{|c|c|c|c|}
\hline Required Input & Variable Type & Required Input & Variable Type \\
\hline Reinforcement Style & Integer & Diameter & Double Float \\
\hline Max Loading & Double Float & Base of column & Double Float \\
\hline Gross Cross-sectional Area & Double Float & Height of column & Double Float \\
\hline $\begin{array}{l}\text { Area of Steel } \\
\text { Reinforcement }\end{array}$ & Double Float & $\begin{array}{c}\text { Concrete Compressive } \\
\text { Strength }\end{array}$ & Double Float \\
\hline Yield Strength of Steel & Double Float & Ply Thickness & Double Float \\
\hline Modulus of FRP & Double Float & $\begin{array}{c}\text { Ultimate Rupture } \\
\text { Strain of FRP }\end{array}$ & Double Float \\
\hline FRP Efficiency Factor & Double Float & & \\
\hline
\end{tabular}

Once the factors are set within the method, an estimated compressive strength of confined column, a local variable, is calculated. This is treated as a local variable because it back calculates the compressive strength using Eq. 2.20 without the strength reduction factor and is not the compressive strength of the confined column because it does not incorporate the area of FRP which is being calculated in the method.

The strength reduction factor for FRP is set to 0.95 as given by $\mathrm{ACl} 440.2 \mathrm{R}-08$ section 12.1 . This is not a recurring variable so it was treated as a local variable.

The method also calculates an estimated confined pressure of the column using Eq. 2.69 This is also a local variable because similarly to the compressive strength, the equation back calculates the pressure without knowing the area of FRP reinforcement and does not represent the actual confined pressure. 
Finally the method calculates the layers needed using Eq. 2.70 based on the above calculated estimated values. The result is a number that is not a whole number and therefore the method then uses the built-in "Math" class and the method "Ceiling" to round the number of layers up to the next whole number. "Ceiling" is used because a partial ply or layer is not practical solution, and to meet the required confined pressure there must be an equivalent or greater number of layers and "Ceiling" meets that criterion.

The method then returns the number of layers to the application as a double float type.

\subsubsection{5 "FactoredAxialCapacity" method}

The last method in the "clsAxialColumnDesign" class is the "FactoredAxialCapacity" method. This method takes into account the number of layers being used and determines the factored capacity of the confined concrete column in pure axial compression. The method inputs are listed in Table. 4.6. Just like in the "UnstrengthenedCapacityofUnstrengthenedColumn" method and the "ConfinedPressure" method the "FactoredAxialCapacity" method determines the strength reduction factor and the eccentricity factor depending on the cross-sectional shape. Also, the strength reduction factor for FRP is also used here as a local variable and set equal to 0.95 .

By using Eq. 2.21, the method calculates the confined concrete column strength which is a local method variable. Afterward the factored axial compressive capacity of the column is calculated using Eq. 2.20 being distinguished by the reinforcement style used in determining the factors. The method then returns the factored capacity to the application as a double float variable. 


\section{Table 4.6 Inputs for "FactoredAxialCapacity" method}

\begin{tabular}{|c|c|}
\hline Required Input & Variable Type \\
\hline Reinforcement Style & Integer \\
\hline Confined Pressure & Double Float \\
\hline Concrete Compressive Strength & Double Float \\
\hline FRP Efficiency Factor & Double Float \\
\hline Gross Cross-sectional Area & Double Float \\
\hline Area of steel reinforcement & Double Float \\
\hline Yield Strength of Steel & Double Float \\
\hline
\end{tabular}

\subsection{6 "clsAxialColumnACIRequirements" class}

This class purpose is to provide methods to calculate any $\mathrm{ACl} 440.2 \mathrm{R}-08$ design requirements. Requirements included confinement, concrete strain and serviceability requirements for the concrete and steel.

\subsubsection{1 “ConfinementRatio" method}

$\mathrm{ACl} 440.2 \mathrm{R}-08$ requires the confinement ratio of the concrete column to be greater than or equal to 0.08 . What this method does is calculates the confinement ratio by dividing the confined pressure by the concrete compressive strength, both of which are required double float inputs for the method. The method does not determine if the confinement ratio meets the $\mathrm{ACl}$ requirement. It only calculates the ratio and returns it to the application as type double float. 


\subsubsection{2 "CompressiveStrainOnConfinedColumn" method}

Another requirement by $\mathrm{ACl}$ is that the maximum compressive strain in the $\mathrm{FRP}$-confined concrete must be less than 0.01 . The "CompressiveStrainOnConfinedColumn" method only determines the induced strain on the confined concrete column by using the Eq. 2.26. The required inputs for the method are located in Table. 4.7.

Before the strain on the confined column can be calculated, the FRP efficiency Factor must first be determined. The method does this by identifying the cross-sectional shape of the column by using the geometry integer. If geometry is equal to 1 , meaning a circular column, the FRP efficiency factor is set to 1 . Else, if the geometry integer is 2 for a non-circular section, Eq. 2.27 is use to calculate the efficiency factor which requires the built-in "Math.Pow" class method for raising the ratio of base and height of the beam to the 0.45 power because there is no power operator available in C\#.

After the FRP efficiency factor is set, the compressive strain is calculated using Eq.2.26 and also uses the built-in class method "Math.Pow" to complete the calculation, and returns the stain as a double float variable to the application if the compressive strain is less than 0.01 else the method sets the strain equal to 0.01 .

\subsubsection{3 "ConcreteStress" method}

The "ConcreteStress" method's purpose is to calculate the stress in the concrete to be compared to the serviceability requirement given by $\mathrm{ACl}$. All required inputs are of double float type and are as follows: dead load, live load, gross cross-sectional area, area of steel reinforcement, modulus of steel, yield strength of steel reinforcement, and the compressive concrete strength. 


\section{Table 4.7 Inputs for "CompressiveStrainOnConfinedColumn" method}

\begin{tabular}{|c|c|}
\hline Required Input & Variable Type \\
\hline Geometry & Integer \\
reinforcement ratio & Double Float \\
\hline Height of Column & Double Float \\
\hline Base of Column & Double Float \\
\hline Confined Pressure & Double Float \\
\hline Concrete Compressive Strength & Double Float \\
\hline Ultimate Rupture Strain of FRP & Double Float \\
\hline
\end{tabular}

By using Eq. 2.56 with respect to axial loading, the method calculates the stress in the concrete but the equation requires values which are not mentioned in the inputs. Eq. 2.56 needs the modulus of the concrete and the serviceability loading condition. The method calculates the modulus using $\mathrm{ACl} 318-$ 08 section 8.5.1. The method does assume that the concrete will be of normal weight. The serviceability loading conditions is just the dead load and live load with no factors. Once those items are determined and the concrete stress is calculated, the concrete stress is returned by the method to the application as a double float variable.

\subsubsection{4 "SteelStress" method}

The last of the methods in the "clsAxialColumnACIRequirements" class is the "SteelStress" method which calculates the stress experienced by the steel reinforcement after confinement to be compared to $\mathrm{ACl}$ limits. The method inputs are of all type double and follows: modulus of steel, gross cross-sectional area, area of steel reinforcement, dead load, live load, and concrete compressive strength. 
Similarly to the "ConcreteStress" method, the procedure is the same expect to the method uses Eq. 2.57 with respect to axial loading to calculate the stress in the steel. The stress is returned as a double float variable.

\subsection{7 "clsInitialStrainOfBeam" class}

The "clsInitialStrainOfBeam" class contains the methods needed to determine the initial strain of a concrete beam before it is strengthened using FRP. The class has methods to calculate the neutral axis of the beam after it cracks, the effective stiffness of a cracked beam, and the initial strain on the beam prior to strengthening.

\subsubsection{1 "NewNeutralAxis" method}

This method calculates the neutral axis for the soffit beam assuming that the beam has cracked. Therefore this method is not the same as "NeutralAxis" method in the "clsOriginalSection" class. "NewNeutralAxis" uses Eq. 2.54 and solves for the neutral axis by applying the quadratic equation. Required inputs for the method are modulus of concrete, modulus of steel, the area of steel reinforcement, the base of the column, and the depth of the tensile steel from the extreme compression side of the section. All inputs are type double float.

Each constant in Eq. 2.54 is calculated individually and used to determine the discriminant. A Ifstatement is used to determine if the discriminant is positive or not. If the discriminant is indeed below zero, signifying a non-real root, the neutral axis will be set to "double.NaN" The double float variable type has a built-in method "NaN" which sets a double float variable to "not a number." When the discriminat is larger than zero, the method will calculate the quadratic equation and set the neutral axis to be returned as a double float type. 


\subsubsection{2 "EffectiveStiffnessOfCrackedBeam" method}

When a concrete beam cracks, the stiffness is affected. Therefore, this method is used to recalculate the stiffness using Eq. 2.55 and assuming the area of FRP is zero. The method needs the following double float inputs: modulus of concrete, base of beam, neutral axis of cracked section, modulus of steel, area of steel reinforcement, and the depth of tensile steel from the extreme compression side of the concrete beam. The effective stiffness is returned as a double float type.

\subsubsection{3 "InitialStrain" method}

The initial strain of the soffit beam can be calculated using the "InitialStrain" method. Eq. 2.59 is implemented and using only the dead load to calculate the initial strain. All inputs are double float variables and include dead load, height of beam, neutral axis of cracked section, and effective stiffness of cracked section. The strain is then returned as a double float to the application.

\subsection{8 "clsFlexureFRPDesign" class}

The "clsFlexureFRPDesign" class is used for determine the number of layers needed to meet required capacity, as well as calculating capacities for determine strengthening configurations, weak and strong, and all other needed methods for calculating capacities and required layers.

\subsubsection{1 "AreaFRPforBSC" method}

In the design process, the balanced strengthening configuration (BSC) is used in determining the minimum number of layers needed to meet the required capacity. The "AreaFRPforBSC" method's main purpose is to determine the total area need to meet that capacity for the balanced configuration.

The method attempts the process by assuming the existence of one layer of FRP. Using that one layer, the effective strain of the FRP for which debonding occurs is calculated using Eq. 2.5. The effective debonding strain is checked to see if it is less than $90 \%$ of the ultimate rupture strain of the FRP. If true, 
the method will set the effective strain to the effective debonding strain. Else, the effective strain will be equal to $90 \%$ of the ultimate rupture strain.

With an effective strain, an initial estimate of the area of FRP is determined first by using Eq.

2.52 which calculates the area of FRP as a function of the additional moment carried by the FRP. Prior to calculating the required area of FRP, a strength reduction factor for FRP is set to 0.85 as suggested by $\mathrm{ACl}$ 440.2R-08 section 10.2.10.

With the addition of a layer of FRP, the neutral axis changes and the strain of the steel reinforcement also changes. The method uses Eq. 2.49 to calculate the adjusted neutral axis and Eq. 2.50 for the strain of the tensile steel reinforcement which is dependent on the neutral axis.

The method will determine the area of FRP needed to meet the balanced strengthening configuration, which depends on if the steel reinforcement is yielding. When the steel does yield, Eq. 2.52 is calculated to determine the area of FRP. If the steel has not yielded, than Eq. 2.53 is used. The method then returns the area of FRP required for the balanced strengthening configuration (BSC) to the application as a double float variable.

\subsubsection{2 "StrainEffective" method}

The "StrainEffective" method is used to calculate the effective strain of the FRP reinforcement. The process is very similar to what is described in section 4.2.8.1 when calculating the effective strain expect instead of the number of layers being preset to 1 in the method, the number of layers is a double float input.

\subsubsection{3 "NumberOfLayers" method}

The purpose of this method is to determine the number of required layers needed to meet the balanced strengthening configuration. The method does assume that the FRP reinforcement will be the 
entire length of the base of the beam. The required inputs area all type double float and are the area of FRP required for balanced strengthening configuration, the ply thickness, and the width of the base of the beam.

By dividing the required area by the the ply thickness and the base of the beam, the number of layers is calculated as a double float. The method then uses the "Math.Round" built-in class method to round the number of layers to 3 significant values. This is done only for display purposes. The rounded double float value is then returned.

\subsubsection{4 "NeutralAxisWSCSteelYeild" method}

The method uses Eq. 2.39 to calculate the neutral axis for when the steel yields for a weak strengthening configuration. All inputs are of double float type and are as follows: area of steel reinforcement, yield strength of steel, area of FRP reinforcement, modulus of FRP, effective strain of FRP, concrete compressive strength, stress block factor, and base of beam. The value returns as a double float variable.

\subsubsection{5 "StrainCompression" method}

The "StrainCompression" method is used to calculate the compression strain on the beam after strengthening by using Eq. 2.45. The required input are all type double: effective strain of FRP, initial strain, the neutral axis, and the height of the beam. The compression strain is retuned as double float type.

\subsubsection{6 "StrainSteelTensileWSC" method}

The strain in the tensile steel of in a weak strengthening configuration (WSC) can be calculated using the "StrainSteelTensileWSC" method. The method uses Eq. 2.47 and needs the effective strain of FRP, the initial strain, the neutral axis, the height of the beam, and the depth of the tensile steel 
reinforcement from the most extreme compression fiber in the beam. All inputs are of double float type, as well as the strain of the tensile steel which is returned to the application.

\subsubsection{6 “NominalMomentCapacityWSCSteelYield" method}

This method will calculate the nominal moment capacity of the strengthened reinforced concrete for when the steel has yielded and when the beam is in a weak strengthening configuration. Eq. 2.12 is used to calculate the capacity while assuming the stress in the steel is equal to the yielding strength of the steel since the steal has been assumed to have yielded.

The required inputs for the method are the area of steel reinforcement, the yield strength of the steel, the depth of the tensile steel with respect to the most extreme compression fiber of the beam, the stress block factor, the neutral axis, the area of FRP, the modulus of FRP, the effective strain of the FRP, and the height of the beam. All inputs and the returned value, nominal moment capacity, are double float variables.

\subsubsection{7 "NeutralAxisWSCSteelNotYield" method}

When the steel has not yielded for a weak strengthening configuration (WSC), the "NeutralAxisWSCSteelNotYield" method can be used to calculate the neutral axis of the beam after strengthening has occurred. Eq. 2.39 with eq. 2.40 and 2.48 are solved using the quadratic equation using the same procedure mentioned in section 4.2.7.1. The method will require the modulus of concrete, the modulus of steel, the area of steel reinforcement, the base of the beam, the depth of the tensile steel with respect to the extreme most compression fiber of the beam. All inputs are type double float as well as the neutral axis which is returned. 


\subsubsection{8 “NominalMomentCapacityWSCSteelNotYield" method}

The method calculates the nominal moment capacity of the strengthened concrete beam in the weak strengthening configuration when the steel reinforcement has not yielded. Similar to "NominalMomentCapacityWSCSteelYield" method, Eq. 2.12 is used, though the stress in the steel is not assumed to be the yield strength because the steel has not yielded. Therefore, the method instead calculates the steel stress using Eq. 2.48 in the nominal capacity equation.

The method requires the following double float inputs: concrete compressive strength, stress block factor, base of the beam, the modulus of steel, the area of steel reinforcement, the effective strain of the FRP, the initial strain of the beam, the modulus of FRP, the area of FRP reinforcement, the height of the beam, depth of the tensile steel with respect to the most extreme compression fiber of the beam, and the neutral axis. After calculating the nominal capacity of the beam after strengthening as a double float type, the method returns the value.

\subsubsection{9 "StrainFRPSSC" method}

The strain experienced by the FRP reinforcement is calculated using the method "StrainFRPSSC." Needing only the height of the beam, the neutral axis, and initial strain of the beam for inputs, all of type double float, the method uses Eq. 2.6 to calculate the FRP strain. Though the Eq. 2.6 uses the effective depth of the reinforcement, the method instead uses the height of the beam because the addition distanced added by the FRP layers is negligible compared to the beam height. The strain is returned to the application as a double float.

\subsubsection{0 "StrainSteelTensileSSC" method}

When the beam is using a strong strengthening configuration (SSC), this method can be used to calculate the strain in the tensile steel. Eq. 2.38 is calculated using just the neutral axis and the depth of 
the tensile steel with respect to most extreme compression fiber of the beam. Both are inputs of double float as well as the strain which is returned.

\subsubsection{1 "NeutralAxisSSCSteelYielding" method}

This method calculates the neutral axis of the strengthened concrete beam for a strong strengthening configuration (SSC) and the steel reinforcement has yielded. Using the quadratic formula to solve Eq. 2.39 with Eq. 2.40, 2.41, and 2.42 and using the same procedure as described in section 4.2.7.1 to solve the quadratic formula, the neutral axis is calculated and returned as a double float type to the application.

\subsubsection{2 “NominalMomentCapacitySSCSteelYield" method}

Using Eq. 2.12, the method calculates the nominal moment capacity of the strengthened concrete beam in the strong strengthening configuration, assuming the steel has yielded.

The method also assumes the steel stress is equivalent to the yield strength and the strain of the FRP is calculated using Eq. 2.37.

The required inputs are the area of steel reinforcement, the yield strength of the steel, the depth of the tensile steel with respect to the most extreme compression fiber of the beam, the stress block factor, the neutral axis, the area of FRP reinforcement, the modulus of FRP, the effective strain of the FRP, and the height of the beam. All values are of type double float including the return value, the nominal moment capacity. 


\subsubsection{3 “NeutralAxisSSCSteelNotYield" method}

The "NeutralAxisSSCSteelNotYield" method calculates the neutral axis of the strengthened concrete beam in the strong strengthening configuration and when the steel reinforcement has been considered to have yielded. The method uses the quadratic equation to solve for the neutral axis using Eq. 2.39 with Eq. 2.43, 2.40, and 2.41 and uses the same process as section 4.2.7.1.

The inputs are the concrete compressive strength, stress block factor, the base of the beam, the modulus of the FRP, the area of FRP reinforcement, the effective strain of the FRP, the initial strain of the beam, the area of steel reinforcement, the yield strength of steel, the height of the beam, and the modulus of steel, and the depth of the tensile steel reinforcement from the extreme compression fiber of the beam. All are type double float including the return value, the neutral axis.

\subsubsection{4 "NominalMomentCapacitySSCSteelNotYield" method}

The method calculates the nominal moment capacity of the strengthened concrete beam that is using a strong strengthening configuration and the steel did not yield. Eq. 2.12 is where the steel stress is a function of the steel strain and the modulus and the FRP strain is calculated using Eq. 2.37.

All the inputs are type double float, and are as follows: the area of the steel reinforcement, the modulus of steel, the depth of the tensile steel with respect to the most extreme compression fiber of the beam, the stress block factor, the neutral axis, the area of FRP reinforcement, the modulus of FRP, the effective strain of the FRP, and the height of the beam. The nominal moment capacity is returned to the application as a double float value.

\subsection{9 "clsFlexureACIRequirements" class}

The "clsFlexureACIRequiremetns" class contains class methods which are used in checking ACl requirements for serviceability limit states for beams undergoing bending. The methods calculate the 
stress level of the FRP, concrete, and steel in both stages of strengthening, prior to the application of FRP and after strengthening occurs.

\subsubsection{1 “NeutralAxisStageB" method}

This method calculates the neutral axis of the strengthened concrete column in stage $B$, which is after the FRP is applied. By solving the quadratic function Eq. 2.54, the method determines the neutral axis using the same procedure as section 4.2.7.1.

The mandatory inputs for the "NeutralAxisStageB" method includ the modulus of concrete, the base of the beam, the modulus of steel, the area of steel reinforcement, the modulus of FRP, the area of FRP reinforcement, the depth of the tensile steel reinforcement with respect to the most extreme compression fiber of the concrete beam, and the height of the beam. All are double float values.

\subsubsection{2 "StressConcrete" method}

The "StressConcrete" method calculates the concrete stress for both stages A and B under serviceability loading. By using Eq. 2.56 without FRP, the method solves for the stress in stage A for the concrete, and the stress in stage B is calculated by using Eq. 2.56 with FRP. Once both stage stresses are calculated, the method then just sums up the two stresses to determine the total concrete stress that will be used to compare to $\mathrm{ACl}$ serviceability requirements.

The method requires the following inputs: the dead load, the live load, the neutral axis, the modulus of concrete, the base of the beam, the modulus of steel, the area of steel reinforcement, the depth of the tensile steel reinforcement with respect to the most extreme compression fiber in the beam, the modulus of FRP, the area of FRP reinforcement, the height of the beam, and the neutral axis of stage B. All inputs and return values are of double float type. 


\subsubsection{3 "StressSteel" method}

The "StressSteel" method calculates the steel stress for both stages A and B under serviceability loading. By using Eq. 2.57 to calculate the stress of the steel in stage A without the FRP, and Eq. 2.57 with the FRP to calculate the stress of the steel in stage $B$, the total stress of the steel in the beam can determined by summing the stress in stages $A$ and $B$.

The method needs identical inputs as the "StressConcrete" method calls for. All area still type double float and the stress of the steel is returned to the application as a double float value.

\subsubsection{4 "StressFRP" method}

The method "StressFRP" can be used to calculate the stress the FRP is experiencing and later be used to check against $\mathrm{ACl}$ requirements. The method only calculates the stress from stage $\mathrm{B}$ because stage $A$ is prior to the addition of FRP strengthening. Therefore there is no FRP to analyze. To calculate the stress in stage B, Eq. 2.60 is implemented.

The method calls for the following inputs of double float type: the steel stress, the modulus of steel, the modulus of FRP, the height of the beam, the depth of the tensile steel with respect to the extreme most compression fiber in the concrete beam, the neutral axis in Stage B, and the initial strain of the beam. The FRP stress is returned as a double float value.

\subsubsection{0 "clsShearDesign" class}

The "clsShearDesign" class provides the application with any required values and calculations needed for the design procedure for shear strengthening using FRP as well as any ACl requirements including maximum allowable shear reinforcement and maximum allowable spacing. 


\subsubsection{1 "StrainEffective" method}

The "StrainEffective" method calculates the maximum strain in the FRP which is limited according to Eq. 2.13 and 2.14. There are only three required inputs: FRP wrapping scheme (style), the ultimate rupture strain of the FRP, and the bond reduction factor.

The FRP wrapping scheme has three different possibilities: four-sided, three-sided, and twosided. Each scheme is represented by an integer. Therefore the FRP wrapping scheme input has to be an integer type. When the input is equal to one for four-sided or otherwise known as complete wrapping, the method will then check to see if $75 \%$ of the ultimate rupture stain is greater than 0.004 . If it is true, the effective strain is set to 0.004 , else it is equal to $75 \%$ of the ultimate rupture stain. When the wrapping scheme is not complete wrapping, meaning the wrapping scheme is either three or two-sided, the method will check if the ultimate rupture strain after being reduced by the bond reduction factor is less than 0.004 . When it is less than 0.004 , the effective strain is Eq. 2.14 , else the effective strain is set to 0.004 .

As previously stated, the wrapping scheme input is of type integer, but the bond reduction factor and the ultimate rupture strain are both double float values as well as the return value, the effective strain.

\subsubsection{2 "RequiredShearCapacityFRP" method}

This method is very simple. Its purpose is to calculate the required shear capacity the FRP. The required inputs are all double float values and include the max loading, the strength reduction factor, the shear capacity of the concrete, the shear capacity of the steel reinforcement, and the strength reduction factor of the FRP. 
To calculate the required shear capacity, Eq. 2.67 is substituted into Eq. 2.62 which is rearranged so the shear capacity can be solved. Eq. 4.1 displays the final result of the rearrangement. The capacity is returned as double float value.

$V_{f}^{R}=\frac{1}{\psi_{f}}\left[\frac{V_{u}}{\Phi}-V_{c}-V_{s}\right]$

\subsubsection{3 "NumberOfLayersContinouslyWrapped" method}

This method can be used to calculate the number of layers of FRP needed to meet the required capacity when the layering is a continuously wrapped along the beam. To calculate an estimate of the number of required layers, the method solves for the number of layers in Eq. 2.67 for continuous wrapping and sets the FRP capacity to the required shear capacity of the FRP.

The inputs required are the required shear capacity of the FRP, the effective strain limit, the depth of FRP, the angle of the FRP, the modulus of the FRP, and the ply thickness. All are type double float. After the number of layers is calculated, it is returned as a double float value.

\subsubsection{4 "ShearCapacityFRPContinousWrapping" method}

When the number of layers being applied is known, the "ShearCapacityFRPContinousWrapping" method can be used to calculate the shear capacity for the FRP when it is continuously applied along the length of the beam. Eq. 2.66 is used to calculate the shear capacity of the FRP.

The method requires the following double float inputs: the number of layers, the ply thickness, the depth of the FRP, the effective strain limit of FRP, the modulus of FRP, and the angle of FRP. The shear capacity of the FRP is returned as a double float value. 


\subsubsection{5 "BondReductionFactor" method}

The purpose of this method is to calculate the bond reduction factor which is needed determining the effective strain limit when the wrapping scheme is either three or two-sided. According to $\mathrm{ACl} 440.2 \mathrm{R}-08$, the bond reduction factor is dependent on two different modification factors and the effective length.

The method will first solve for the effective length using Eq. 2.16. The method also defines a variable called "fraction" to be two-thirds. This was done to eliminate an error occurring when using the "Math.Pow" class method where two-thirds is the power. With "fraction" being set, the first modification factor is calculated using Eq. 2.17.

Next the wrapping scheme is checked to see if it is equal to 2 which stand for three-sided wrapping. When it is equal to 2, the second modification factor is calculated using Eq. 2.18 for " $\mathrm{U}$ wraps." If the scheme does not equal 2, then the method assumes that scheme to be two-sided and calculates Eq. 2.18 for "two sides bonded."

With both modification factors and the effective length calculated, the method finally determines the bond reduction factor by using Eq. 2.15. The reduction factor is then checked to see if it is over 0.75 . If it is over 0.75 , the bond reduction factor is set to be equal to 0.75 .

The required inputs include an integer value for the wrapping scheme, and double float values for the following inputs: concrete compressive strength, number of layers, ply thickness, modulus of FRP, ultimate rupture strain of FRP, and depth of FRP. The bond reduction factor is returned as a double float value. 


\subsubsection{6 "MaxShearSpacingFRP" method}

When the shear FRP reinforcement is not continuously wrapped along the length of the beam, this method can be used to calculate the maximum allowable spacing between strips of FRP reinforcement as designated by $\mathrm{ACl}$ 318-08. Eq. 2.64 is rearranged to solve for the spacing of the FRP reinforcement assuming the shear capacity to be the required shear capacity of the FRP.

All inputs for the method are double float type and are as follows: number of layers, ply thickness, width of FRP, depth of FRP, the effective strain limit, the modulus of FRP, the required shear capacity of the FRP. The max spacing allowed is returned as a double float type to the application.

\subsubsection{7 “NumberOfLayersDiscontinousWrapping" method}

This method is used when the wrapping scheme is discontinuous along the length of the beam meaning that FRP reinforcement is applied in intermittent strips. The number of layers is calculated similarly to a continuous scheme mentioned in section 4.2.10.4 expect Eq. 2.64 is used instead of Eq. 13.92.

The following are required inputs of type double float: shear spacing of the FRP, the required shear capacity of the FRP, the ply thickness, the width of FRP, the depth of FRP, the effective strain limit of the FRP, the modulus of FRP, and the angle of FRP. After calculated the number of layers for a discontinuous strengthening system, the number of layers is returned as a double float value.

\subsubsection{8 "ShearCapcityFRPDiscontinousWrapping" method}

The shear capacity of the FRP reinforcement for a discontinuous wrapping scheme can be calculated by using the "ShearCapacityFRPDiscontinousWrapping" method. The method uses Eq. 2.64 to calculate the shear capacity of the FRP. 
The method inputs required are the number of layers, ply thickness, width of FRP, effective strain limit of FRP, modulus of FRP, angle of FRP, depth of FRP, and the spacing of the FRP strips. All inputs are of type double float as well as the return value, the shear capacity of the FRP.

\subsubsection{9 "ShearReinforcement" method}

The purpose of the "ShearReinforcement" method is to calculate the sum of the shear capacity of the steel and FRP reinforcement to be checked against $\mathrm{ACl} 440.2 \mathrm{R}-08$ requirements. The method requires only the shear capacity of the steel and the shear capacity of the FRP, both of type double float. The shear reinforcement is returned also as a double float value.

\subsubsection{0 "MaxAllowableShearReinforcement" method}

The maximum allowable shear reinforcement limit can be calculated using this method. The method uses the Eq. 2.19 to determine the allowable limit. The inputs are all double float values and included the concrete compressive strength, the base of the beam, and the depth of the tensile steel with respect to the most extreme compression fiber of the beam. After calculating the allowable shear reinforcement, the method returns it as a double float value.

\subsubsection{1 "clsBeamColumns" class}

The "clsBeamColumns" class provides all required methods to calculate values for strengthened and non-strengthened members, as well as factored and non-factored axial and bending capacities for specific locations on the interaction diagram which is used for verifying the design.

\subsubsection{1 "TotalAreaOfSteel" method}

The "TotalAreaOfSteel" sums the area of longitudinal steel reinforcement. The method is required because of how the steel reinforcement is entered by the user. The steel is separated by layers. 
Therefore, the total area of steel is not given by the user, but is required for several calculations for the interaction diagram, hence the purpose of the method.

There is only one input for the method, the area of steel as a "list" of type double float. The "list" class is a built-in class that is an array of undefined length of double float values. This allows for the array to become larger when the user puts in multiple layers of steel reinforcement.

There are two local variables defined. The first is a double float variable called "totalAreaOfSteel" which will eventually be the value that is returned. The second is an integer value called " $\mathrm{i}$ " which is used as a dummy counter.

The method calculates the total area of steel by using a for-loop. For every for-loop, there are three parameters. The first parameter defines what variable is being defined. In this case, "i" is being set to zero stating that the loop will first run with "i" equaling to zero. The second parameter checks to see if a conditional statement is true. If it is true, the loop continues, else the loop terminates. For this method the conditional statement set is "i" is less than "areaOfSteel.Count." The "areaOfSteel.Count" class method counts the number of entries in the "list" array "areaOfSteel." Therefore the loop will continue when " $\mathrm{i}$ " is less than the total number of entries in the entered array. When the second parameter is true, the third parameter is applied to the loop. The third parameter's purpose is to change the value of the defined variable for the loop. So, when " $i$ " is less than the count, the loop will add one to the previous "i" value.

So, when the for-loop is running, it begins an iterative process of adding up the values of the "list" area. It takes the value of "totalAreaOfSteel" and adds the value located at the i'th place to total. For example, when " $\mathrm{i}$ " is equal to zero, the total area of steel is initial equal to zero and the O'th place area, which is the first value in the array because of how C\# defines arrays. This process continues until 
the count of entries in the array is equal to "i" after which the total area of steel is returned as a double float value to the application.

\subsubsection{2 "StrainEffective" method}

The "StrainEffective" method is used to determine the limit of the effective strain of the FRP using Eq. 2.28. The only input required for this method is the ultimate rupture strain of the FRP as a double float type.

The method checks to see if $55 \%$ of the ultimate rupture strain is less than 0.004 . If true, the effective strain limit is $55 \%$ of the ultimate rupture strain. If it is false, the effective strain is set to 0.004 . After the effective strain limit is determined, it is returned as a double float value.

\subsubsection{3 "ConfinedPressure" method}

The confined pressure of the beam-column can be calculated using the "ConfinedPressure" method. The method will use Eq. 2.22 to determine the confined pressure caused by the FRP reinforcement, but first the appropriate diameter must be determined.

This must be done because a beam-column may have a non-circular cross-section. The input "geometry," an integer type, defines the cross-section. If it is equal to 2 , then the cross-section is noncircular and the method calculates the diameter using Eq. 2.24. Afterward the confined pressure is calculated and returned as a double float type.

The following are all additional required inputs for the method: modulus of FRP, number of layers, ply thickness, diameter, effective strain limit, base of member, and height of member.

\subsubsection{4 "UltimateAxialCompressionStrainOfConfinedConcrete" method}

The objective of this method is to determine the maximum compressive strain in the FRPconfined concrete. The method is similar to section 4.2.5.2 when calculating "AeAc" and 4.2.6.2 when 
calculating the maximum compressive strain which is determined using Eq. 2.26. The maximum compressive strain in the confined concrete is returned as a double float value to the application.

\subsubsection{5 "ConfinedConcreteCompressiveStrength" method}

To calculate the confined compressive strength of the concrete, the "ConfinedConcreteCompressiveStrength" method should be used. The method uses the same process to calculate "AeAc" value as in section 4.2.5.2. For calculating the confined concrete compressive strength, Eq. 2.21 is implemented.

The required inputs for the method are the concrete compressive strength, confined pressure, geometry, base of the member, height of the member, total area of steel, and the gross cross-sectional area of the member. All are type double float except for geometry which is an integer value. The confined compressive strength is retuned as a type double float.

\subsubsection{6 "StressBlockParameterA" method}

This method's objective is to determine the stress block parameter A to be used in the beamcolumn interaction diagram. The parameter is function of the concrete compressive strength, the ratio of confined compressive strength to the unconfined concrete compressive strength, and the compression strain of the confined concrete. The method uses tabular values provided by Barbero [4] to determine the parameter through a bilinear interpolation dependent on the ratio of concrete strengths and the compression strain of the concrete.

There are only three inputs required for the method, all type double float: concrete compressive strength, confined concrete compressive strength, and the axial compression strain of confined concrete. The value will return the stress block parameter A as a double float value. 
Before the return value is calculated the method process with tabular values to use and interpolate those values to calculate the parameter. The first thing that is done is the defining of all local variables required for the bilinear interpolation. The axes of the tabulated charts are defined with the top axis as a double float array, representing the ratio of strengths, starting from 1 and ending at 3 in increments of 0.2. The side axis is a double float array, for confined concrete strain, with values ranging from 0.0035 to 0.01 in increments of 0.0005 . Two additional arrays are defined, "tempArrayX" and "tempArrayY." Both are empty arrays that are the length of the corresponding axis array. An empty 14 by 11 matrix is also defined. Five integer values are defined: "X1", "X2", "Y1", "Y2", and "i". The first four integer values are set to zero. There are also five double float values defined: "Q11", "Q21", "Q12", "Q22", and "tempnumber." The purpose of these local variables will be discussed later in the section.

The tabulated values provided correlation to a unique strength of concrete. Therefore, the method will first determine which table to use by checking the concrete compressive strength versus the strength corresponding to the table. Since Barbero [4] only provides three different strengths, the method checks to see if the compressive strength is equal to $20.68,27.57$, or 34.47 and then sets the empty 14 by 11 matrix equal to the corresponding tabulated table.

From this table and the predefined axes, a bilinear interpolation can be done, but unfortunately there is no built-in class method that could handle this request. Therefore, a unique process was created to find the required values ("Q11", “Q12", “Q21", "Q22") for the interpolation which has been visually represented in Figure 4.3. 


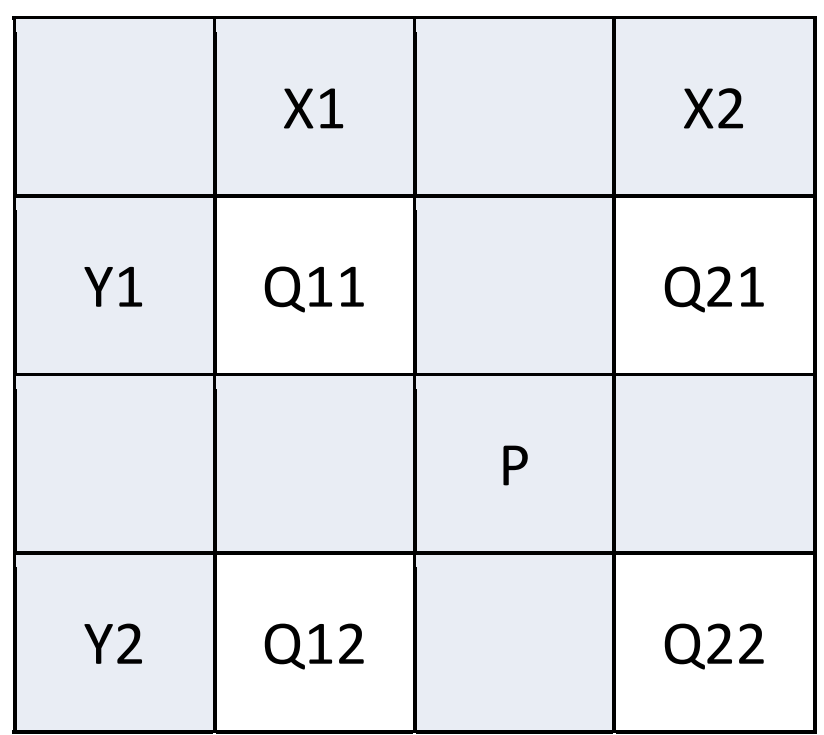

Figure 4.2 Bilinear Interpolation Diagram

The initial step is to determine " $\mathrm{X} 1$ " and " $\mathrm{X} 2$ " which are the values in the confined and unconfined strength ratio before and after the calculated ratio using the entered values. A for-loop is initiated with " $\mathrm{i}$ " equaling to zero for the first parameter because the first index in an array is zero. The second parameter is the conditional that " $\mathrm{i}$ " is less than the length of the top axis array. The last parameter adds one to the previous value of "i."

To determine if a value on the top axis of the table is below or above the calculated ratio, the for-loop takes the current index of the top axis array and subtracts it from the calculated ratio and adds it to the "tempArrayX" at the same index. Therefore if the values of the "tempArrayX" are below zero, it means the top axis array value at that corresponding index is below the calculated value. If the value of the "tempArrayX" is above, it means the top axis array value at that index is above the calculated value. The "tempArrayX" value calculated for that current interval is set to the "tempnumber" variable to be used later in determining indexes.

Next the for-loop will check to see if " $i$ " is greater than zero. If it is not, meaning that it is the first entry in the array, then " $\mathrm{X} 1$ " is set to the index where "tempnumber" is equal to a value in the 
"tempArrayX" array. This is done by using the built-in class method

"Array.IndexOf<double>(tempArrayX, tempnumber)." If "i" is indeed larger than zero, then the loop will check if the latest value of "tempArrayX" is less than zero and if the latest value is greater than the previous value of "tempArrayX". This is a critical check because in order to perform the bilinear interpolation, the values immediately below the calculated ratio are needed, and this checks to see that the latest value is smaller than the calculated value but still larger than any calculated value with a lower indexes. When these conditionals are true, "X1" is set to the index of the current installment of "tempArrayX."

If either of the two conditionals is false, then the loop will check to see if the value is the immediate value above the calculated ratio. To do this the conditional statements checks to see if the previous value in "tempArrayX" is less than the current value and if the previous value is greater than zero. This looks to make sure the value is above the calculated ratio and to make sure that the smallest of these values is being used. When either is not true, the loop sets " $\mathrm{X} 2$ " to the current index of "tempnumber" in "tempArrayX." This should always happen when the calculated difference between values in the top axis and the calculated ratio, and therefore making the second set of conditional statements a redundancy, but is still kept as a safe guard against potential changes in the arrays. So when the second set of if-statements are true, the loop then checks to see if " $\mathrm{X} 2$ " is equal to zero and if it is, it sets "X2" equal to the previous index of "tempArrayX" that was calculated.

The same procedure is used for the side axis and " $\mathrm{Y}$ " values and Figure 4.4 shows the programming of the method for procedure. 


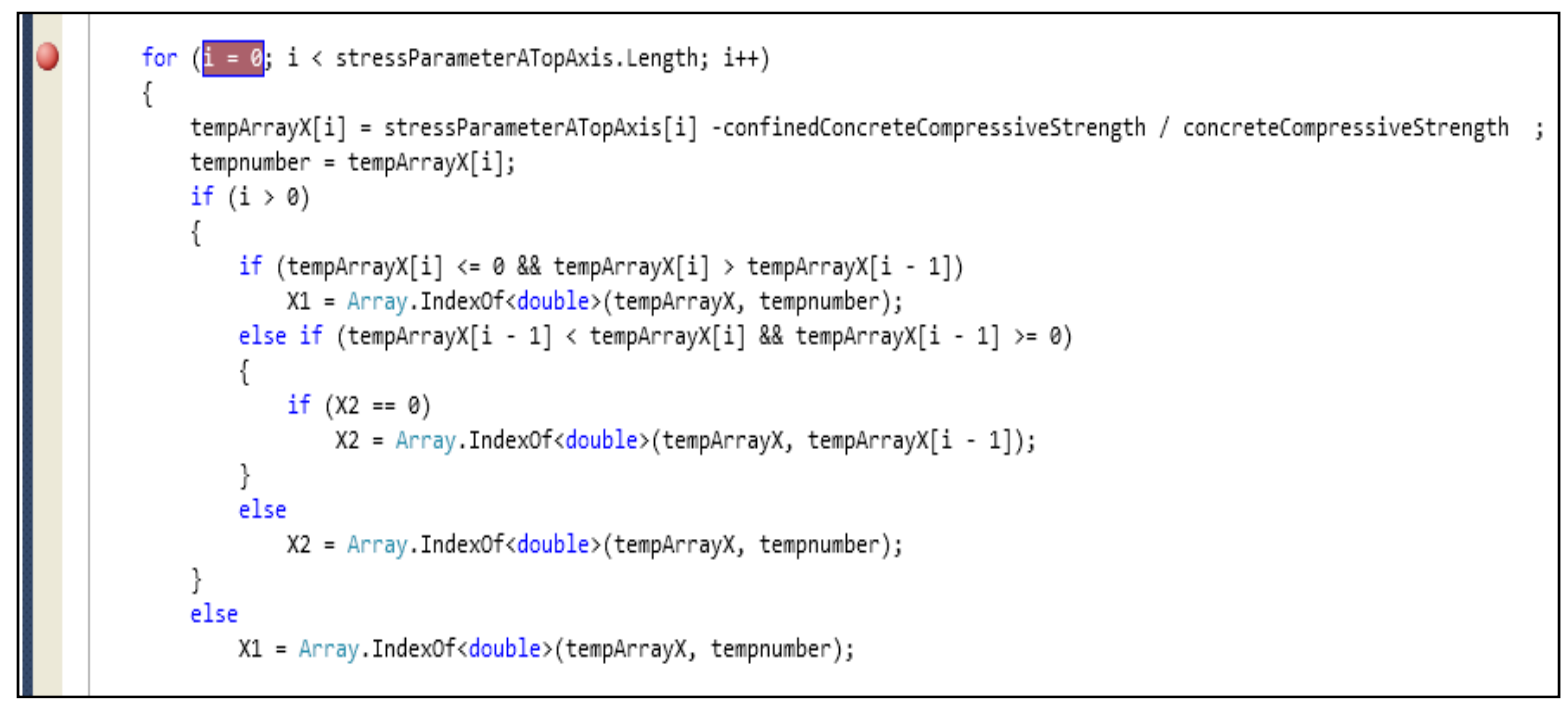

Figure 4.3 Bilinear Interpolation X-axis procedures

Once "X1", "X2", "Y1", and "Y2" are determined, the intermediate " $Q$ " values can be looked up in the tabulated table defined earlier. The " $\mathrm{X}$ " and " $\mathrm{Y}$ " values are indexes which together produce an index within a matrix. Therefore, using the names of the " $Q$ " variables, the proper values can be assigned. For example, "Q21" is set to the corresponding value located at "X2" and "Y1". Figure 4.4 can further illustrate this concept. Also, " $\mathrm{X}$ " and " $\mathrm{Y}$ " are now locally defined as the ratio of confined concrete compressive strength and the unconfined concrete compressive strength, and the axial compression strain on the confined concrete, respectively. This was done to easy the length of the bilinear interpolation equation. Finally, the bilinear interpolation can be done to produce the stress block parameter $\mathrm{A}$.

\subsubsection{7 "StressBlockParameterB" method}

The "StressBlockParameterB" method is identical as the "StressBlockParameterA" method expect for the tabulated tables have changed and are provided by Barbero [4]. The tables are still dependent on the ratio of strength, the compression strain, and the concrete compressive strength. 


\subsubsection{8 "PointA" method}

This method calculates the value at point $A$ which is when the beam-column is in pure axial compression for any of the four plots. The method checks to see which plot point is to be calculated based on the check integer input. If check equals 1, for non-factored strengthened member, Eq. 2.20 is used without the strength reduction factor with the compression strength being equal to the confined compression strength. If check equals 2 for factored strengthened member, Eq. 2.20 is used with the reduction factor. If check equals 3, non-factored and non-strengthened member, Eq. 2.20 is used with no strength reduction factor and the compression strength is equal to the unconfined strength. If check equals 4, for factored non-strengthened member, Eq. 2.20 is used again but with the strength reduction factored added.

The following inputs are also required and are all double float values: the confined concrete compressive strength, the gross cross-sectional area, the total area of steel, the yield strength of the steel reinforcement, the concrete compressive strength, the strength reduction factor and the eccentricity factor. When Point $A$ is calculated, it is returned as a double float value.

\subsubsection{9 "PointD" method}

Point $\mathrm{D}$ in the interaction diagram for beam-columns is calculated using the "PointD" method. Point $\mathrm{D}$ in the diagram is where a minimum eccentricity of $10 \%$ of the height of the member is introduced to Point A values. The method checks to see which plot the point is being calculated similarly to "PointA" method. The method requires the following double float inputs: point A axial strength, point A non-strengthen strength, height of the member, and the strength reduction factor. Also, the check integer input is required. The method returns Point $D$ as a double float value. 


\subsubsection{0 "StrainSteel" method}

The purpose of the method is to calculate the strain the steel reinforcement is experiencing for a given layer of steel reinforcement. There are two integer value inputs required for the method: "i" and " $n$ " and the importance of these values will be explain in section 4.2.11.12.

The method first checks to see if " $i$ " is equal to " $n$ " and if this is the case, then it checks to see if the ultimate steel strain is equal to zero. When this is true, the steel strain is equal to zero. Else when "i" does not equal "n," the steel strain is equal to Eq. 2.72. The steel strain is returned as a double float value.

Other required inputs include the ultimate concrete strain, the neutral axis, the concrete compressive strength, the distance of the current layer of steel reinforcement, and the ultimate steel strain. All of these inputs are of double float type.

\subsubsection{1 "StressSteel" method}

The "StressSteel" method determines the stress of the steel with respect to the steel strain to the ultimate steel strain. The method first checks to see if the steel strain is greater than the ultimate strain. If true, the steel stress is equal to the yield strength of the steel because it assumes the steel has yielded. Else, if the steel strain is less than the negative value of the yield strength, then the steel stress is the negative value of the yield strength. When neither of the conditional statements is valid, the method calculates the steel stress using Hook's law. This piecewise system is shown in Eq. 2.73.

The method requires the following double float inputs: the strain of steel, the ultimate strain of steel, the modulus of steel, and the yield strength of the steel reinforcement. The steel stress is returned as a double float value. 


\subsubsection{2 "PointBorC" method}

Since the procedure for both Point $B$ and Point $C$ of the interaction diagram are the same, one method was used. The method determines the axial and bending capacities for all four plots by calculating the internal compression force and the stress resultants of the steel reinforcement layers. Eq. 2.75 and 2.76 are used to calculate the capacities.

First, the method defines all the local variables, but more importantly, this method also defines a local class object which is called "beamcolumnPointBorC" which is a "clsBeamColumn" class. This allows for the method to use this object and any class methods associated with the object's class.

The neutral axis is calculated by using Eq. 2.71. For point B, the ultimate steel strain should be equal to zero. With the neutral axis determined, the method checks to see if it is calculating an axial capacity or a moment capacity by using the input integer check. 1, 2, 5, and 6 all represent the confined members while 3, 4, 7, 8 means members are unconfined. The procedures for both cases are very similar with the single difference being how the concrete compressive stress block is calculated.

If either conditional statement is true, the local variables total stress resultant and tensile stress block are set to zero, followed by the calculation of the concrete compressive stress block. Eq. 2.40 with the confined stress block parameters is used when the member is confined. If it is not confined, then Eq. 2.40 is used instead.

After the stress block is calculated a for-loop is initiated with the local integer " $i$ " being set to zero for the first parameter. The second parameter is the conditional statement that "i" is less than the total number of entries of the "areaOfSteel" array, and the last parameter adds one to the current value of "i." During the loop, the class method "beamColumnPointBorC.StrainSteel" is called. For more information on this method please refer to section 4.2.11.10. The method is called to calculate the steel strain for the first layer of steel reinforcement. The following values are used in the inputs for the 
"beamColumnPointBorC.StrainSteel" method: the ultimate concrete strain, the neutral axis, the concrete compressive stress block, the distance of steel at index " $\mathrm{i}$ ", the ultimate steel strain, " $\mathrm{i}$ ", and the number of entries in the "distanceOfSteel" array. The steel strain method does account for appropriated strains such as the closest layer of steel reinforcement to the tensile fiber for Point B being equal to zero.

The loop continues by calling the method "StressSteel" which is described in section 4.2.11.11. The steel stress returned by "StressSteel" method is then added to the previous total steel resultant value. The tensile stress block as seen in Eq. 2.76 is also calculated and added to the total pervious value of the tensile stress block.

After the loop terminates, the method checks to see what plot value needs to be calculated. For check values of 1 or 3, Eq. 2.75 is used. For check values of 2 or 4, Eq. 2.75 is multiplied by the strength reduction factor. Eq. 2.76 is used for $5,6,7$, and 8 , but when check equals 6 or 8 , the strength reduction factor is also applied.

The required inputs for the method can be seen in Table 4.8.

\subsubsection{3 "PointE" method}

This method calculates Point E of the interaction diagram for any of the four plots. The method calculates Point $\mathrm{E}$ by using an iterative procedure for when the eccentricity of the beam-column system is equal to 100 . The method first defines any local variables to be used and creates a class object "BeamColumnPointE" as a "clsBeamColumns" class. 
Table 4.8 Inputs for "PointBorC" and "PointE" methods

\begin{tabular}{|c|c|c|c|c|c|}
\hline $\begin{array}{l}\text { Required } \\
\text { Inputs }\end{array}$ & Variable Type & $\begin{array}{l}\text { Required } \\
\text { Inputs }\end{array}$ & Variable Type & $\begin{array}{l}\text { Required } \\
\text { Inputs }\end{array}$ & Variable Type \\
\hline $\begin{array}{c}\text { Ultimate } \\
\text { concrete strain }\end{array}$ & Double float & $\begin{array}{c}\text { Concrete } \\
\text { compressive } \\
\text { strength }\end{array}$ & Double float & $\begin{array}{l}\text { Confined } \\
\text { concrete } \\
\text { strength }\end{array}$ & Double float \\
\hline Yield Strength & Double float & $\begin{array}{l}\text { Base of } \\
\text { member }\end{array}$ & Double float & $\begin{array}{c}\text { Ultimate steel } \\
\text { strain }\end{array}$ & Double float \\
\hline $\begin{array}{c}\text { Depth of } \\
\text { Tensile Steel }\end{array}$ & Double float & $\begin{array}{l}\text { Distance of } \\
\text { steel }\end{array}$ & List <double> & $\begin{array}{c}\text { Axial } \\
\text { compression } \\
\text { strain of } \\
\text { confined } \\
\text { concrete }\end{array}$ & Double float \\
\hline $\begin{array}{l}\text { Stress block } \\
\text { factor }\end{array}$ & Double Float & Area of steel & List < double > & $\begin{array}{c}\text { Modulus of } \\
\text { steel }\end{array}$ & Double float \\
\hline Check & Integer & $\begin{array}{l}\text { Height of } \\
\text { member }\end{array}$ & Double float & $\begin{array}{l}\text { Strength } \\
\text { reduction } \\
\text { factor }\end{array}$ & Double float \\
\hline $\begin{array}{l}\text { Stress block } \\
\text { parameter A }\end{array}$ & Double float & $\begin{array}{l}\text { Stress block } \\
\text { Parameter B }\end{array}$ & Double float & & \\
\hline
\end{tabular}

Afterwards, an assumed value of 0.6 for " $g$ " is set. " $g$ " is the ratio of the neutral axis and the depth of the tensile steel reinforcement. This value is used to vary the neutral axis during the iterative process. Also the "error" variable is set to zero.

The error was set to zero so when the method runs the while-loop, the loop will initiate. The while-loop unlike the for-loop will run until the conditional statement is false. For this while-loop, while the error is greater than 1.01 or the error is less than 0.99 , the loop will continue to run.

So while the loop is running, the neutral axis is calculated by multiplying the depth of the tensile reinforcement with the "g" value. The method then checks to see if it is calculating a confined member or non-confined member depending on the value of the check integer. This is done in the same manner as "PointBorC" method mentions in section 4.2.11.12 except after the moment and axial capacities are calculated the method determine the eccentricity by dividing the moment capacity by the axial capacity. 
With the new eccentricity, a new error is calculated around the value 100. If the error is less than 0.999 the " $g$ " value is reduced by 0.00001 . Else if the error is greater than 1.001 , then the " $g$ " value is increased by 0.00001 . This continues until the while-loop condition is false.

All the required inputs can be seen in Table 4.8. The value of Point $E$ is returned as a double float type.

\subsubsection{4 "PointF" method}

The "PointF" method calculates the values at Point $F$ in the interaction diagram which is considered to be in pure flexure. The initial variables are defined including a list $<$ double $>$ called sort which equals the distance of steel array. The sort "list" is used to arrange the values of the distance of steel list provided by in ascending order.

First the method uses the class method "Sort" to achieve the rearrangement. Then a temporary variable is used to store the last item in the sort array. That temporary value is looked up in the original distances of steel array to produce an index to be used to find the value of corresponding area of steel for that layer. This process provides the area of steel for the tensile steel reinforcement.

The area of steel for the compression steel reinforcement must also be determined. Since the sort array is arranged in ascending order, the initial value of the array at index 0 is equal to a temporary value. Then the index of that temporary value is looked up in the original distance of steel reinforcement and with that index the area of steel for the compression layer is called.

With these areas determined, Eq. 2.77 is used with the area of FRP equal to zero and a new term for the compressive steel reinforcement. This term is developed by using similar triangles of a strain diagram for a double reinforced section [6]. The quadratic equation is used to solve Eq. 13.42. The procedure is similar to section 4.2.7.1. With a neutral axis calculated, the steel compression strain is 
calculated by using the new term. The steel stress in compression is calculated by multiplying the modulus of steel by the steel compression strain. The strain of steel in tension is also calculated by multiplying the modulus by the tensile steel strain.

The method then checks to see if the plot point is factored or not. When not factored, Point $F$ is equal to Eq. 2.78 with area of FRP is equal to zero. When it is factored, the same is equation is used with applying the strength reduction factor.

All inputs can be seen in Table 4.9. Point $F$ is returned as a double float.

Table 4.9 Inputs for "PointF" method

\begin{tabular}{|c|c|c|c|c|c|}
\hline $\begin{array}{c}\text { Required } \\
\text { Inputs }\end{array}$ & Variable Type & Require Inputs & Variable Type & $\begin{array}{c}\text { Required } \\
\text { Inputs }\end{array}$ & Variable Type \\
\hline Area of steel & List<double> & $\begin{array}{c}\text { Concrete } \\
\text { compressive } \\
\text { strength }\end{array}$ & Double float & $\begin{array}{c}\text { Stress block } \\
\text { factor }\end{array}$ & Double float \\
\hline $\begin{array}{c}\text { Base of } \\
\text { member }\end{array}$ & Double float & $\begin{array}{c}\text { Modulus of } \\
\text { steel }\end{array}$ & Double float & $\begin{array}{c}\text { Ultimate } \\
\text { concrete strain }\end{array}$ & Double float \\
\hline $\begin{array}{c}\text { Distance of } \\
\text { steel }\end{array}$ & List<double> & $\begin{array}{c}\text { Strength } \\
\text { reduction } \\
\text { factor }\end{array}$ & Double float & $\begin{array}{c}\text { Depth of } \\
\text { tensile steel }\end{array}$ & Double float \\
\hline Check & Integer & & & & \\
\hline
\end{tabular}




\subsection{Windows Forms Design}

To execute the design process for strengthening of reinforced concrete by using FRP, the Windows Form application uses what are known as forms. These forms help move the user through stages of the design by allowing the user to interact through buttons and enabled textboxes for entering required information. Through these interactions, the form uses the classes and methods designed described in Section 4.2 to implement the design process. The majority of the decision making and procedures used for design are done within the form's programming. Therefore, this section will explain the programming of each form from the interacting with the user and entered values, to the design process implemented within the background of the application.

\subsubsection{Form structure}

Each form has a similar structure composed of four major sections. The first major section is dedicated to only defining all required global variables and objects to be used within the form. Items included here range from variables such the diameter of the column or the clear cover of the reinforcing steel to all objects that are implemented including any buttons, textboxes, and labels. Another point of importance is whether the item is public or private. When an item is declared to be private that means only within the form is the object allowed to be changed, but if it is considered a public object than it is allowing other classes and forms to manipulate the values and objects in the form.

The second section of the form structure is the Initialization component. The purpose of this section is developing the interface for the user. The form code requires this so the form can be built when the application is run. For any object on the window form, it will show up here with addition items informing the location of the object, if the object is enabled, any texts associated with the object, the color of the object, and when there is an event associated with the object, the instance is created here. 
It will be assumed that user has basic $\mathrm{C} \#$ knowledge, and therefore this section of the form will be skipped when discussing individual forms.

The third section found in every form is the form constructor. The constructor is what the form first does when it is called by another form or method. The constructor behaves in a similar manner to a method but its main purpose is to generate the form. It does this by calling the Initialize Component section of the form, and if there is anything else the form must do when it is immediately built, this is where it is located. Only constructors that do more than just initialize components will be discussed.

The fourth section of the form structure deals with any event handlers. An event handler is used when the form is to do something after the user has initiated an event. Events could be the act of clicking a button on the window form to losing focus on a textbox. Nevertheless, when these actions take place, the form will run the event handler. These event handlers are where majority of the design process takes place and each form will have a variety of handlers doing unique operations.

\subsection{2 "frmMain"}

When the application first opens, the Main Form, "frmMain", opens as seen in Figure 3.1. The purpose of this form is to direct the user to the appropriate path depending on what type of member will be strengthened. Therefore, three buttons were needed for each member type: beam, column, and beam-column. Each of these buttons has an event handler for when the user clicks the button. There are two critical items defined in the first section of the form. There is a fourth button called "btnClose." It also has an event handler which will explain in section 4.4.2.4. Lastly, a single variable called "membertype" which is an integer value is defined. The purpose of this value is to keep track of which member was selected to be used in the "frmLoading" form. 


\subsubsection{1 "frmMain" Event Handler "btnBeam_Click"}

The "btnBeam_Click" handler is used when the user clicks the Beam button. The handler creates a new object from the "frmBeam" form and uses the build-in method to show this new object and as well to hide the current form.

\subsubsection{2 "frmMain" Event Handler "btnColumn_Click"}

This event handler proceeds in the same manner as "btnBeam_Click" but it creates a object from the "frmLoading" form with the input of "membertype" which was set to be equal to 3 in the event handler. The three will be used to convey to "frmLoading" that the use has selected the column member to be strengthened.

\subsubsection{3 "frmMain" Event Handler "btnBeamColumn_Click"}

The same process is done in this event handler as section 4.4.2.1 except it will open a new "frmBeamColumnProperties" from instead.

\subsubsection{4 "frmMain" Event Handler "btnClose_Click"}

When the user clicks the close button, the event handler will close the form and exit the application. This will be the case for every close button within the application.

\subsection{3 "frmLoading"}

The "frmLoading", Figure 3.3, form's purpose is to collect loads and determine the maximum loading combination using Eq. 2.29 to Eq. 2.35. The form is designed to have an enabled textbox for each variety of loading which are all defined in the first section of the form structure. The form has two buttons: back and continue, each with event handler. Also, the form constructor takes into consideration the member type. Therefore member type has been defined as integer as well. 


\subsubsection{1 "frmLoading" constructor}

The form constructor does more than just initialize components of the form because the "frmLoading" is used by every member type expect beam-columns. Therefore, each time the form is constructed, the labels that displays the units for the loading varieties changes by using a switch-case. The switch case looks to see what the member type integer is equal to. When the case is equal to 1 , all of the unit labels are changed to " $\mathrm{N}-\mathrm{mm}$ " for moment loading or when the case is equal to 2 or 3 , the labels are changed to " $\mathrm{N}$ " for axial or shearing loads depending on the member type. This a must so the user is a aware of the required units the inputs must be for the all design process are dependent on the a particularly unit for all inputs.

For the form to know what member type has been selected, the constructor requires an input that is an integer value. So, when "frmMain" or "frmBeam" creates a new loading form, they are required to input an integer value for the switch-case to examine.

\subsubsection{2 "frmLoading" event handler "btnBack_Click"}

If the user clicks the back button on the window form, the event handler will create a new Main Form for the user to start from by closing the current window form. This is the same procedure that is used for every back button within the application. Therefore all back buttons returns the user to the Main Form.

\subsubsection{3 "frmLoading" event handler "btnContinue_Click"}

The event handler first defines all local variables needed to complete all actions within the event. A double float object is created for all loads as well as for the max loading combination, and for the minimum and maximum values to validate the loading inputs. Also, a Boolean value called "flag" is also defined. This Boolean value is used for anytime "double.TryParse" method is used so it can be checked to see if it is false. 
The event handler will create a new object class "Loading" using the class "clsLoading" which is described in section 4.3.1.

After all the needed objects have been defined, the handler will convert all the input values in the textboxes to double float numbers and check to see input is a number and if is within the allowable range. Figure 4.5 demonstrates the general approach for all of these types of checks throughout the application.

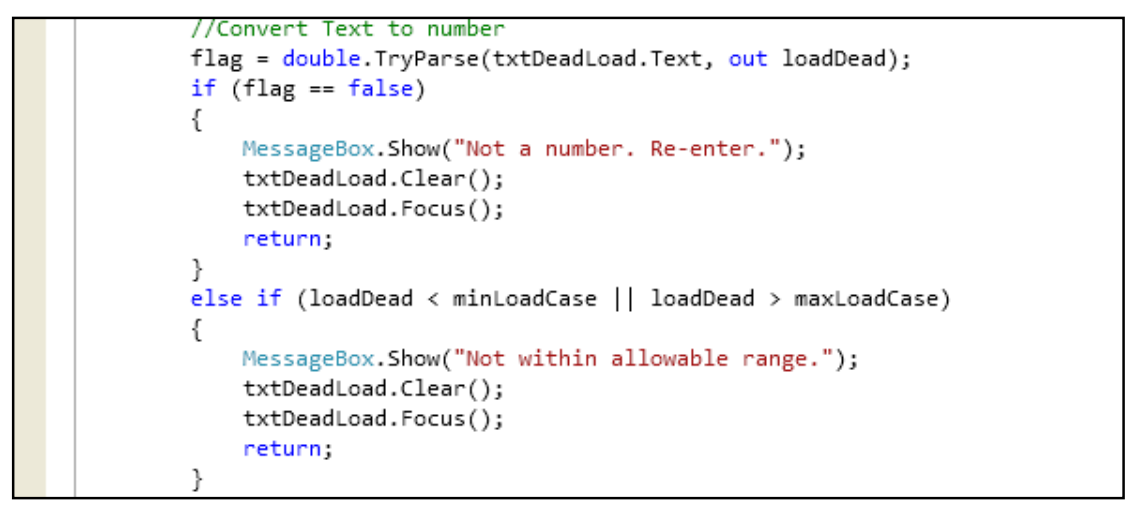

\section{Figure 4.4 General Structure for checking for valid user inputs}

This general structure for checking inputs will first try to convert the input text to a number by using the "double.TryParse" method. This method will take the text from the given textbox, convert it to a number and set the given variable equal to that number while setting the Boolean "flag" equal to true if the conversion was successful or false when the input was not a number.

When "flag" is false, the handler will create a message box informing the user that an input was not a number. Afterwards it will clear and focus in on the textbox that failed this check, and return to the current form.

The check must also look to see if the converted text is within an allowable range by using an else-if conditional statement. The statement will see if the converted number is below a minimum value or above a maximum value. If either case is false, the handler will produce a message box informing the 
user the value was not within the allowable range and clears and focuses on the textbox in question. Then the handler returns the user back to the current form.

After all the textbox inputs have been validated, the handler calculates the maximum loading combination but using the "MaximumLoadingScenerio" method described in section 4.3.1. This value will be used as the required strength of the concrete member.

With a max loading combination, the handler advances the application to the appropriate form depending on the member type integer. If member type equals 1 for beams in flexure, the handler will open a new "frmFlexureBeamProporties" form and hide the current form. When member type is 2 for beams in shear, a new "frmShearBeamProperties" form is open and the current form is hidden. Lastly if member type is equal to 3 for columns in pure axial compression, the handler will open "frmColumnProperties" form and close the current form. All three forms require the max loading combination, the dead load, and the live load.

\subsection{4 "frmBeams"}

The "frmBeam" from, Figure 3.2, is used to distinguish how the user plans to strengthen the concrete beam. Three buttons are located on the windows form, two buttons for flexure strengthening or shear strengthening, and the last as a back button. The event handlers for the strengthening buttons will use the same procedure as section 4.3.2.2 except the member type when flexure is select is equal to 1 and when shear is selected is equal to 2 . The back button is the same as stated in section 4.3.3.2.

\subsection{5 "frmFlexureBeamProperties"}

This form is used to collect all required geometry and material properties need to complete the design for strengthening of beams for flexure (Figure 3.5). The form has what are known as group boxes. A group box is created for five different set of properties: geometry, steel, FRP, exposure, and concrete. 
The group box allows for objects within the box to behave in a conjoining manner which is particularly useful when using radio buttons for fiber type and exposure conditions. The form also defines all maximum and minimums for each textbox inputs.

There are only two buttons. One is a back button used return the user to the Main Form. The other button is a continue button with a unique event handler.

\subsubsection{1 "frmFlexureBeamProperties" event handler "btnContinue_Click"}

This event handler will check all textbox inputs in the same manner as described in section

4.3.3.3. The statues of the radio button also checked. The handler will see which radio button is checked for fiber types and exposure conditions. Figure 4.6 demonstrates the general process for checking radio buttons. The fiber type is equal to 1,2 , or 3 when the radio button for carbon, glass, or aramid is selected, respectively. If a radio button is not selected, the handler will create a message box that asks the use to select a fiber type and then focus on the carbon radio button. The exposure conditions are done in the same fashion. The condition is equal to 1 for interior conditions, 2 for exterior conditions, and 3 for aggressive conditions.

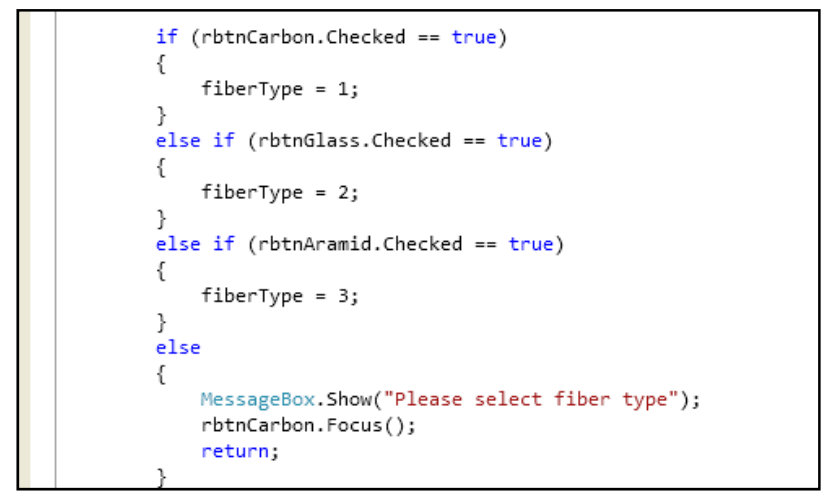

Figure 4.5 General structure of checking radio buttons 
The "btnContinue_Click" event handler does create a new local class from "clsFRPEnvironmentalFactor" and uses the method "environmentnalReductionFactor" to calculate the environmental reduction factor using the fiber type an exposure.

Once all the inputs are validated, the event handler will then create a new "frmFlexureResults" form. Once the form is created, all of the public variables in "frmFlexureResults" are updated using the converted input texts given by the user. Afterwards the new form is open and the current form is hidden.

\subsection{6 "frmFlexureResults"}

The form is used to display and process the design of the strengthened beam in flexure as well as any calculated values and display required checks (Figure 3.6 and 3.8). All variables that are user inputs are defined as public in the form to allow other sources to access and update the values. The rest of the variables defined in the form are used within the form only. Five object classes (“clsOriginalSection”, “clsInitialStrain”, "clsFRPsystem”, "clsFlexureFRPDesign”, "clsFlexureACIRequirements") are created initially too, each representing a unique step in the design process.

There are four buttons on the form (Figure 3.6). The Back and Close button functions the same as stated in earlier sections. The Calculated Results button is used to initiate the design process and complete all design calculations prior to user entering the number of layers of FRP to be used. The Continue button is used after the user has entered the number layers to finish the design process and complete all $\mathrm{ACl}$ requirements. 


\subsubsection{1 "frmFlexureResults" event handler "btnCalculateResults_Click"}

The event handler will complete the first few steps of the design process for strengthening a reinforced concrete member.

First, the initial moment capacity, the required moment capacity, and the maximum allowable reinforcement need to be calculated. The required moment capacity should have been determined in the "frmLoading" form and passed forward in the process, but the capacity of the original section is unknown. To calculate the capacity, the handler must first solve for the stress block factor, the neutral axis, and the depth of the tensile steel. "StressBlockFactor" method, section 4.3.4.6, is called and returns the stress block factor. The neutral axis is calculated using the "NeutralAxis" method from section 4.3.4.7. The depth of the tensile steel reinforcement is calculated by subtracting the clear cover from the height of the beam. With these three items solved, the moment capacity of the original beam is calculated by using "MomentCapacityOfOriginalSection" method, section 4.3.4.8. The handler then finds the factored capacity by multiplying the original capacity by the strength reduction factor which is assumed to be 0.9 .

The purpose for calculating the original moment capacity is to see if it meets the minimum loading capacity and if it meets maximum allowable reinforcement. The minimum loading capacity is calculated using the "MinimumLoadCapacity" method, section 4.3.4.1. To compare allowable reinforcement, the maximum allowable and the actual percent of strengthening must be calculated. The event handler calls "MaxPercentActual" and "MaxPercentAllowable" methods to return the percents needed for comparison.

With this four values determined (minimum loading requirement, original moment capacity, percent actual strengthening, max allowable strengthening) the handler finally compares them by using if-statements. First the original moment capacity is checked to see if it is greater than the minimum 
loading requirement. If false, the textbox for this requirement is updated to say that the beam didn't meet the minimum loading requirement and changes the minimum strength checkbox to false. If it is true, then the textbox for minimum strength is updated to inform the user it has met the requirements and the checkbox is true. Also, when the moment capacity is greater than the minimum loading, the percents are checked to see if the actual percent of strengthening is less than the allowable percent. If true, then the max allowable reinforcement textbox informs the user of meeting the requirement, and the allowable reinforcement checkbox is true. Else, if it is not true, the same textbox display that the user failed the requirement and the checkbox is equal to false.

The second stage of the design process for beams in flexure is to determine the initial strain at the soffit of the beam and the initial stress distribution before strengthening. In order to calculate the initial strain the position of the neutral axis of for when the section is cracked is needed. Therefore, the handler will calculate the modulus of the concrete according to $\mathrm{ACl} 318$ section 8.5 , and use the method "NewNeutralAxis" as discussed in section 4.3.7.1. Because the method does use the quadratic formula to solve for the neutral axis, it must check to see if the method returns a non-number. If it does the event handler will create a message box explaining there are no real roots for the solution and return to the form.

The effective stiffness is also needed and is found using "EffectiveStiffnessOfCrackedBeam" method, section 4.3.7.2. With the neutral axis and the effective stiffness, the initial strain of the concrete beam is calculated using the method "InitialStrain" described in section 4.3.7.3.

The third stage is determining the preliminary design quantities regarding the FRP. The ultimate tensile strength, the ultimate rupture strain, and the modulus of the FRP are calculated using methods from section 4.3.3. 
With all the preliminary design calculations done, the fourth stage of the design process begins in this event handler. An estimated area of FRP need for the balance strengthening configuration is determine using "AreaFRPforBSC" method, section 4.3.8.1, and therefore an estimate for the number of layers needed, assuming the FRP to be applied to the entire base of the beam, can be calculated using "FlexureFRPDesign.NumberOfLayers" class method, section 4.3.8.3. That double float value is converted to a string and displayed to the user in the "txtRequiredNumberOfLayers" textbox. The event handler then checks to see if the two checkboxes for the initial requirements are both true. If both are true, the textboxes for user inputs for the width and number of FRP layers are enabled for the user to use.

\subsubsection{2 "frmFlexureResults" event handler "btnContinue_Click"}

This event handler occurs when the user clicks the continue button. Hopefully, this is done after the user inputs a number of layers and a width for the FRP. Nevertheless, the inputs are checked in a similar manner as Figure 4.5 expect the it will check to see if the user entered a width greater than the beam instead of checking against a range.

If both are valid inputs, the number of layers is converted to a double float value called layers. This is because the handler checks to see if the input for the number of layers is an integer to prevent the user from using a terminating decimal because a fraction of a ply is not applicable. With the double float layers, the total area of FRP is calculated by multiplying the ply thickness and the width by the number of layers. This concludes step 5 of the design.

Step 6 determines the moment capacity of the strengthened concrete beam and verifies to see if it meets the required capacity governed by the loading conditions. Prior to any checking of the configuration, the effective strain is recalculated using "FlexureFRPDesign.StrainEffective" again with updated values. Now the event handler determines what type of configuration was selected by comparing the area of FRP applied to the area of FRP needed to meet the balance strengthening 
configuration (BSC). When the area applied is less than the BSC area, then the event handler knows that the beam will be using a weak strengthening configuration (WSC). The other option is when the area applied is greater. This produces a strong strengthening configuration (SSC) for the beam.

If the beam is in WSC, the textbox dedicated for the configuration is changed to WSC, and the design stage to find the strengthened concrete beam capacity begins. First, the failure mode of the beam should be checked to see if it is tension controlled. Therefore the event handler calculates the neutral axis when the steel reinforcement has yielded by using "NeutralAxisWSCSteelYeild" method and calculates the strain of the tensile steel using "StrainSteelTensileWSC" method. According to ACI 318, when the strain of the steel is greater or equal to 0.005 , this considered to be in a tension controlled state. Therefore, the strength reduction factor is 0.9 and the steel is assumed to yield before the concrete fails.

If the beam is in a tension controlled state, the nominal moment capacity is calculated using "NominalMomentCapacityWSCSteelYeild" method. The compression strain is also determined by using the method "StrainCompression." With the nominal capacity and the strength reduction factor, the factored moment capacity is calculated and then changed to a string value to be used by the "txtFactorCapacity" textbox to be displayed to the user. Also, the required loading is converted to a string for the "txtRequiredCapacity" textbox. The handler then compares these two capacities to see if the strengthen beam is strong enough to meet the requirements. If so, the textbox informing the user displays "Meets Required Moment Capacity" and the associated checkbox is set to true. Else if the factored capacity is less than the required capacity, the textbox informs the user the beam failed to meet the required capacity and changes the checkbox to false.

Now, it is possible for the concrete beam to not be in tension controlled state, but rather be in a transitional or compression controlled state. So when the steel strain is less than 0.005 , a new neutral 
axis is calculated using "NeutralAxisWSCSteelNotYield" which solves a quadratic formula to find the neutral axis. The event handler handles this in the same manner as described in section 4.3.6.1. With the neutral axis, the nominal moment capacity is determined by the method

"NominalMomentCapacityWSCSteelNotYield." To factor the nominal capacity the strength reduction factor needs to be determined. Since the beam is not in a tension controlled state, the strength reduction factor is not 0.9 , but rather a function of the steel strain and the ultimate steel strain. If the strain is less than the ultimate strain, the strength reduction factor is set to 0.65 . Else, the strength reduction factor is calculated based on $\mathrm{ACl} 318$ section 9.3.2. With the new strength reduction factor the procedure is identical to when the steel yielded for the beam in a WSC.

When the area of FRP selected by the user is greater than the area needed for the balance strengthening configuration, the beam is considered to be in a strong strengthening configuration (SSC). So if this is true, the event handler begins the design process to calculate the moment capacity in a nearly identical procedure as for WSC, expect different methods are used to calculate the same value. First the neutral axis is calculated with the assumption that the steel yielded and using the "NeutralAxisSSCSteelYield" method which solves a quadratic formula and therefore checks the return the values for a non-number. The strain steel is determined using the "StrainSteelTensileSSC" method and used to see if the beam is in a tension controlled state. If true, "NominalMomentCapacitySSCSteelYield" method returns the nominal capacity of the concrete beam. The rest of the process is identical to WSC in tension controlled state expect the strain of the FRP is calculated using "StrainFRPSSC" and the compression strain is set to 0.003 .

If the SSC is not in a tension controlled state the same process as WSC not in a tension controlled state is followed using the appropriate methods for SSC with the addition of the FRP strain. "NeutralAxisSSCSteelNotYielding" returns the neutral axis. "NominalMomentCapacitySSCSteelNotYield" 
is used to find the nominal capacity. The steel strain is recalculated using "StrainSteelTensileSSC." The rest of the process is identical to WSC when not in tension controlled state.

The event handler also handles the last step of the design process which is checking ACI requirements. This is always done when the continue button is clicked no matter if the beam meets required capacity. $\mathrm{ACl} 440.2 \mathrm{R}-08$ requires the stress of the concrete, steel, and FRP be under a certain limit which varies depending on the material. But before any stress is calculated, the neutral axis of the beam after the FRP is applied needs to be calculated by using "NeutralAxisStageB" method. The method solves a quadratic equation and needs to be checked to see if it is a non-number as described earlier. With the neutral axis, the concrete stress, steel stress, and FRP stress are calculated using "StressConcrete," "StressSteel," and "StressFRP" methods, respectively. The max stress are $45 \%$ of the concrete strength, $80 \%$ of the steel yield strength, and $55 \%$ of the ultimate tensile strength of the FRP.

The handler will check each of these stresses against the max allowable stress. If the stress meets the requirement, the associated textbox is updated to say "Meets allowable stress levels" and checkbox is equal to true. Else if the stress doesn't meet the requirement, the same textbox will say "Does not meet allowable stress level" and the checkbox equals false.

Finally, the event handler looks to see if all the checkboxes on the form equal true. If so, a message box pops up informing the user that the design meets all requirements. If not, the message box will say "You have failed some requirements. You will need to redesign." (Figure 3.8)

\subsection{7 "frmShearProperties"}

This form is used to collect all required properties needed to complete the design process for strengthening reinforced concrete beams for shearing (Figure 3.9). The form has the same values and design as the flexure properties form with an additional input, spacing between steel reinforcement. There are still five group boxes for each type of material and exposure conditions. The spacing of 
reinforcement is located in the steel properties group box. Also, all maximum and minimum values for every input are defined prior to any event handlers are called. There are only two buttons on the form. The Back button, and the Continue button.

\subsubsection{1 "frmShearProperties" event handler "btnContinue_Click"}

This event handler has the same purpose as the "btnContinue_Click" handler for

"frmFlexureBeamProperties." Every input given by the user will be checked to verify it is a number and then set that value to a defined variable using the "double.TryPharse" method as seen in Figure 4.5 which includes checking to see if the input value is within allowable ranges.

The radio buttons are also checked in the same manner as Figure 4.6 and with radio button values given, the environmental reduction factor is returned from the "EnvironmentalFactor" method which becomes passed along to the "frmShearResults" form along with all relevant values needed for the design process.

\subsection{8 "frmShearResults"}

The "frmShearResults" takes the all the public values given from the "frmShearProperties" and begins the design process for strengthening for shear (Figure 3.10 and 3.11). Since the design process requires several other factors in how to apply the FRP reinforcement, the group box for FRP layers has subgroup boxes containing radio buttons to ask the user how the FRP is going to be wrapped and if the wrapping is continuous or discontinuous along the length of the beam. Because of these options, several textboxes within the FRP layers group box will be enabled or disabled using event handlers.

The form does create three new class objects which are used for the design process. The "clsOriginalSection" class is used to help in determining initial capacities and values of the beam. For 
finding preliminary values for the FRP system, "clsFRPsystem" object class is created. Lastly, the design process of the shearing reinforcement will take advantage of the "clsShearDesign" class methods.

\subsubsection{1 "frmShearResults" constructor}

The form has a unique constructor not only initializes all components of the window form and define any inputs required by the form constructor, the form updates the textbox for the FRP to 90 (Figure 3.11). This is just a default value for the angle the FRP is applied. Also the constructor uses a message box to inform the user they must select a FRP wrapping option before calculating results. The message shows up prior to the form. The wrapping scheme is needed in order to determine how many layers of FRP will be needed, hence why the message box informs the user to select first.

\subsubsection{2 "frmShearResults" event handler "rbtComplete_CheckedChanged"}

When the user changes the status to the complete wrapping scheme button in the subgroup box indicating that the user plans to confine the entire beam with FRP, an event handler is activated to check if the complete radio button is indeed selected. If the radio button is selected, the textbox for the depth of the FRP is updated to display the height of the beam after converting the value to a string as well as disenable the textbox so the user can't input a value. The style integer is set to 1 and the FRP strength reduction factor is set to 0.95 .

\subsubsection{3 "frmShearResults" event handler "rbt3Sided_CheckedChanged"}

When the status of the 3-sided radio button has changed, the event handler checks the status of the radio button. If the status informs the handler that the radio button has been selected, the textbox for the depth of the wrapping is enabled so the user may input a value. The style integer is equal to 2 and the FRP strength reduction factor is 0.85 . 


\subsubsection{4 "frmShearResults" event handler "rbt2Sided_CheckedChanged"}

When the status of the 2-sided radio button has changed, the event handler checks the status of the radio button. When the radio status says it has been selected, the textbox for the depth of the FRP reinforcement is enabled. The style integer is equal to 3, and the FRP strength reduction factor is equal to 0.85 .

\subsubsection{5 "frmShearResults" event handler "rbtContinous_CheckedChanged"}

When the status of the Continuous radio button has changed, the event handler checks to see if the status states the button has been selected. When the button is selected, the textbox of the width of the FRP and the spacing of the FRP become disenabled, and both text boxes are updated to show "N/A." the textbox for the angle for the FRP reinforcement is also disenabled, but text is updated to show 90 because the wrapping is continuous meaning that applying the FRP reinforcement at an angle is impracticable and is assumed to be applied in a vertical manner to the beam with respect to the beam.

\subsubsection{6 "frmShearResults" event handler "rbtDiscontinous_CheckedChanged"}

When the status of the radio button for discontinuous wrapping of the beam has changed, the event handler checks to see if the discontinuous radio button has been selected. If the button has been selected, the textboxes for the width, spacing, and angle are all cleared with the width and angle textboxes also being enabled for the user to input values. The spacing is not enabled because if the preliminary design steps have not been completed, a maximum allowable spacing isn't available to compare the input value to, and therefore the enabling waits till the allowable spacing has been determined. 


\subsubsection{7 "frmShearResults" event handler "btnCalculateResults_Clicked"}

The design process begins when the user clicks the Calculate button. The event handler determines the initial capacity of the beam for shearing and checks it against the minimum loading capacity and the maximum allowable strengthening.

Before the factored shear capacity of the beam is calculated and compared to the minimum loading capacity, several class methods are used to calculate required values. First the depth of the tensile steel is determined by subtracting the clear cover from the height of the beam. Then, the minimum loading capacity is calculated using "MinimumLoadCapacityOfUnStrengthenedColumn." The shear capacity of the concrete is calculated using "ShearCapacityConcrete" and the capacity of the steel reinforcement is calculated using "ShearCapacitySteel." The strength reduction factor is set equal to 0.75 as given by $\mathrm{ACl} 318$ section 9.3.2.3 for shear strength. With these values the factored capacity is determined using "ShearFactoredCapacityBeam" method.

The maximum allowable and actual percent of strengthening is determined using the factored shear capacity, minimum loading capacity, and the required loading capacity by using the "MaxPercentActual" and "MaxPercentAllowable" methods.

With all values needed to confirm the requirements of allowable strengthening and minimum capacity, the textboxes for the associated values are updated with the corresponding variables. The event handler then checks to see if the factored capacity is greater than the minimum capacity required and process in an identical manner as described in section 4.3.6.1 when dealing with checking the maximum allowable strengthening and minimum loading requirements.

After determining the initial capacity of the beam, the preliminary values for the FRP system are calculated using the "UltimateTensileStrengthOfFRP," "UltimateRuptureStrainOfFRP," and 
"ModulusOfFRP" methods are used to calculate the ultimate tensile strength, ultimate rupture strain, and modulus of the FRP.

Before the required number of layers are determined, the event handler must first determine which wrapping scheme is being used and obtain the required values for the scheme. Therefore, the wrapping scheme radio buttons are checked to see if they are selected, first with the complete radio button. If the complete radio button is indeed selected, the depth of the FRP is equal to the height of the beam. Else if the radio button for 3-sided wrapping is selected, the input for the depth of the FRP is converted to a value and checked to make sure the input is a value. The process is the same as shown in Figure 4.5 except the limits on the input value is the height of the beam, and the message box reflects the excess of the input. Else, if the 2-sided radio button is selected, the depth of the FRP input is checked in the identical manner as for the 3-sided scheme. Lastly, if none of the buttons are checked, the textbox for the depth of the FRP and the Continue Button is disenabled.

After determining the wrapping style, the input for the angle of the FRP is converted to a double float value and under goes the same check as any input. When the Boolean variable is true, the angle of the FRP is converted to radians because all design equations are radian dependent.

Finally the event handler begins the process for determining the number of layers required to meet the required shear capacity. First a local variable called "styleLocal" is set to 1 to reflect a complete wrapping scheme. This is a required assumption in order to calculate a bonding reduction factor. With the local style equal to 1 , the effective strain limit is determining using "StrainEffective" with the bond reduction factor equal to zero. The required shear capacity of the FRP is calculated using the "RequiredShearCapacityFRP" method. With the required capacity of the FRP and the effective strain, a first estimate of the number of layers needed is determined by using the "NumberOfLayersContinouslyWrapped" method, and therefore assuming a continuously wrapped 
beam. The output raised to the next whole number because a partial layer is not practical. Now with preliminary number layers needed for a completely wrapped continuous FRP shear reinforcement, a more appropriate bond reduction factor is calculated using "BondReductionFactor" method in order to obtain a better effective strain limit and the number of layers needed to meet the required capacity of the beam.

The number of layers is once again raised to the next whole number and converted to a string to be displayed to the user in the appropriate textbox. The textbox for the user to input the number of layers is also enabled.

\subsubsection{8 "frmShearResults" event handler "txtUserInputNumberOfLayers_TextChanged"}

When the user does input a number of layers to be used on the concrete beam, the event handler checks if the wrapping is continuous or discontinuous and checks the required inputs are valid, and determine the maximum allowable spacing of the FRP reinforcement if needed be.

The first thing the event handler will do is convert the number of layers into a type integer and check to see if it is a valid input in a similar process as seen in Figure 4.5. Next the radio buttons are checked to see if one is selected. If the continuous radio button is selected, the angle of FRP is equal to 90 and then converted to radians. The Continue button is enabled. The number of layers is converted to a type double float value. If the discontinuous radio button is selected, the width of the FRP is enabled, cleared, and focused. The inputs of the depth and angle of the FRP are validated, and if the angle is validated, the process will assume that the beam will be continuously wrapped to determine the maximum allowable spacing of the FRP reinforcement by converting the angle to radians, and the number of layers to a double float type. The maximum spacing is calculated using the 
"MaxShearSpacingFRP" method. The return value is then converted to a string and displayed in the "txtMaxShearSpacing" textbox. The spacing textbox is enabled as well as the Continue button.

If neither radio button has been selected, the event handler will disenable the textboxes for with width and spacing of the FRP and the Continue button. A message box will pop up indicating that the user needs to select if the beam will be continuously or discontinuously wrapped and to resubmit.

\subsubsection{9 "frmShearResults" event handler "btnContinue_Click"}

To complete the final design process for shear strengthening, the Continue button is clicked to calculate the shear capacity of the strengthened beam and to check any $\mathrm{ACl}$ requirements. First the handler sees if the beam is discontinuously wrapped. If so, the input for the spacing of the FRP reinforcement must be validated using the same process as seen in Figure 4.5 expect the limiting value is just the max shear spacing.

Next the capacity can be calculated, but two different methods are used depending if the beam is continuously or discontinuously wrapped. If the beam is continuously wrapped, the capacity is calculated by using the "ShearCapacityFRPContinousWrapping" method. Else, if the beam is discontinuously wrapped, the method "ShearCapacityFRPDiscontinouslyWrapping" is used to determine the shear capacity. With the capacity known, it is converted to a string in displayed in the window form in the "txtFactoredCapacity" textbox, and the required capacity is also displayed to the user. The capacity is then checked against the required capacity. If greater, the form updates the corresponding textbox and checkbox. Else, those boxes will reflect the failure to meet the required capacity.

The only $\mathrm{ACl}$ requirement to satisfy is the maximum allowable shear reinforcement. The method "MaxAllowableShearReinforcement" method is used to calculate the maximum allowable while the shear reinforcement of the beam is calculated using the "ShearReinforcement" method. Both values are converted to strings and displayed, then compared to see if the shear reinforcement is less or equal to 
the maximum allowable. If true, the textbox to display the result is updated to say it meets the requirements and the checkbox is changed to true. Else, the textbox displays to the user that the requirement was not met, and the checkbox is changed to false.

Lastly, the event handler sees if all check boxes are true. If true, a message box appears and tells the user the design met all requirements. Else, the message box will inform the user that the design failed some requirements and needs to be redesigned.

\subsection{9 "frmColumnProperties"}

This form is used to gather user inputs for required variables needed to complete the design process for strengthening reinforced concrete columns (Figure 3.12). The majority of the inputs within the form are similar to the other properties forms. The Geometric properties group box now has two radio buttons for the shape of the cross-sectional area which is needed first find the gross crosssectional area of the column and also determine several factors related to the shape. Also a diameter and gross cross-sectional area also have textboxes in the geometric properties group box. The only other group box that is different is the steel properties group box. There is no longer a textbox for neither clear cover nor shear spacing of the reinforcement. Instead, there are two radio buttons for the user to select the style of steel reinforcement, tie or spiral. The selection will dictate several factors within the design process. There are only two buttons on the window form, Back and Continue.

There are several event handlers for the form because of the cross-sectional shape. Depending on the selection of the shape, an event handler will enable or disenable the diameter, base of column, and height of column textboxes which each have a handler for determining the gross cross-sectional area. Also, the Back button behaves like the other back buttons in the application, and the event handler for the Continue button is explained in section 4.3.9.6. 


\subsubsection{1 "frmColumnProperties" event handler "rbtnCircular_CheckedChanged"}

If the status of the radio button for circular changes, the event handler will check to see if the status is selected. When the radio is selected, the textboxes for the base and height of the column become read-only and the text is updated to zero. The textbox for the diameter becomes enabled, cleared, focused, and the no longer read-only.

When the radio button is not selected, the textboxes have a reversal of behavior. The diameter textbox becomes read-only and disenabled reading just zero. The height and base of the column textboxes are enabled, cleared, focused, and no longer read-only.

\subsubsection{2 "frmColumnProperties" event handler "rbtnRectangular_CheckedChanged"}

The event handler is extremely similar to "rbtnCircular_CheckedChanged" except it behaves in the opposite manner. When the radio button for rectangular cross-section is selected, the event handler does the identical procedure as when the radio button for circular cross-sectional is not selected. The same goes for when the rectangular radio button is not selected the event handler does the same thing as when the circular radio button is selected. Refer to section 4.3.9.1.

\subsubsection{3 "frmColunProperties" event handler "txtDiameter_LostFocus"}

The purpose of this event handler is to calculate the gross cross-sectional area of the column when the diameter textbox is no longer in focus. The event handler first defines the Pi and the outer and inner limits of the diameter.

Next, the status of the circular radio button is checked to see if it is currently selected. This is to make sure to only calculate the gross cross-sectional area using the diameter if the radio button has been selected because the event handler is activated when the textbox losses focus, not when the text changes. Therefore the user could focus in on the textbox even when it is disenabled and the event 
handler will still run after the focus is lost. So if the circular button has been selected, the diameter input is validated in the same process as seen in Figure 4.5. Then the gross cross-sectional area is calculated by simply calculating the area of a circle. This value is then converted to a string to be shown in the diameter textbox. Also the geometry integer is set equal to 1 to represent a circular cross-section.

\subsubsection{4 "frmColumnProperties" event handler "txtBaseOfColumn_LostFocus"}

The event handler will activate when the textbox for the base of the column loses focus. When it does lose focus, the gross cross-sectional is calculated using the base and height of the column. First the upper and lower limits of the base and height are defined using the "double.TryParse" method.

Afterwards, the height textbox is checked to see if the textbox is not null or empty which means it checks to see if there was an input in the height textbox. If there was, then it will validate both the height and base inputs using the procedure seen in Figure 4.5. If both are valid inputs and have been converted to double float values, the gross cross-sectional area is calculated by determining the area of a rectangle. The value is converted to a string and displayed in the gross area textbox. The geometry integer is set equal to 2 to represent a non-circular cross-section.

\subsubsection{5 "frmColumnProperties" event handler "txtHeightOfColumn_LostFocus"}

The purpose of this event handler is the same as section 4.3.9.4 and the process is the same for when the height of column textbox loses focus, but instead of checking the height textbox to see if it is null or empty, the event handler will check the base textbox to make sure it is not empty or null. If it is not, the same validation and calculates as discussed in section 4.3.9.4 take place. The reason for having two different event handlers that run the same process is to make sure that no matter which value the user inputs first, that the gross cross-sectional area is always calculated. 


\subsubsection{6 "frmColumnProperties" event handler "btnContinue_Clicked"}

For when the user clicks the Continue button (Figure 3.12), the event handler process is similar to 4.3.7.1 and 4.3.5.1. The event handler first defines all local variables and upper and lower limits for all inputs. The exposure radio buttons and fiber radio buttons are checked in the same manner as Figure 4.6 and all user inputs are validated using Figure 4.5 structure. The environmental reduction factor is calculated using the "EnvironmentalFactor" method. Finally the form "frmColumnResults" is created and all the new form's required public variables for design purposes are defined. Afterward the new form (see Figure 3.13) is shown as the current form is hidden.

\subsubsection{0 "frmColumnResults"}

The "frmColumnResults" form is to use the inputs from "frmColumnProperties" form to run the design process for strengthening reinforced concrete columns (Figure 3.13). The layout of the window form is similar as beams with initial checks, FRP layers, and Final checks group boxes. There are four buttons on the window form: Back, Close, Calculate Results, and Continue. The Back and Close buttons both function as all other Back and Close buttons in the application. The Calculate Results button is used to begin the design process by calculating preliminary values and initial estimates of required FRP. The Continue button is to be used after the user inputs the number of layers of FRP that will be applied, and the event handler will then finish the design process by validating the capacity and all $\mathrm{ACl}$ requirements.

\subsubsection{1 "frmColumnResults" event handler "btnCalculateResults"}

When the user clicks the Calculate Results button, the initial design steps begin with first step being determining the factored axial strength of the unconfined concrete column to be checked against the minimum load requirements which is calculated by using the "MinimuLoadCapacityOfUnstrengthenedColumn" method. The factored capacity of the unconfined column is determined using the "UnstrengthenedCapacityOfUnstrengthenedColumn" method. With 
these values, the maximum allowable and actual strengthening of the column can also be calculated so they can later be compared. These are determined by using the "MaxPercentActual" and "MaxPercentAllowable" methods.

All of these values are then converted to strings and displayed in the corresponding textboxes in the Windows Form so the user can see the calculated results of these methods. Then unconfined capacity and the actual strengthening checked in the same procedure as 4.3.6.1.

The preliminary values for the FRP system is calculated using the "clsFRPsystem" class methods to calculate the ultimate tensile strength and rupture strain as well as the modulus of the FRP.

Next the longitudinal steel reinforcement ratio and the FRP efficiency factor are calculated by "AEAC" and "FRPEfficiencyFactor" methods to be used in determining the number of required layers of FRP is needed to meet the required loading capacity. Before the "RequiredNumberOfLayers" method is implemented, the event handler checks to see if the cross-section is non-circular, and if true, a diameter is calculated using Eq. 2.24 because the method requires a diameter.

With a diameter, "RequiredNumberOfLayers" returns the value which is then converted to string to be displayed to the user. Lastly, the event handler then checks to see if the two checkboxes for minimum loading requirements and maximum allowable strengthening are both true. If so, the textbox for entering the number of layers is enabled for the user.

\subsubsection{2 "frmColumnResults" event handler "btnContinue_Clicked"}

After the user inputs an integer in for the number of layers being applied and clicks the Continue button, the event handler runs the final steps of the design process for strengthening reinforced concrete columns. 
First, the handler validates the entered number of layers. If the input is valid, then the integer value given is converted to a double float, and the process for calculating the confined concrete column capacity begins by first calculating the confined pressure of the column by using the "ConfinedPressure" method. The confined pressure is needed for the "FactoredAxialCapacity" method used to determine the capacity of the confined column. The returned value from the method is converted to a string and displayed to the user. Then the capacity is compared to the required load capacity. Just like any other comparisons during the design phase, the corresponding textbox is updated with information that the column meets or does not met the required capacity while the checkbox is changed to true or false depending on the outcome.

$\mathrm{ACl}$ has several requirements for confining concrete columns with FRP which included a confinement ratio, compression strain limit, and concrete and steel stress limits. First, the event handler recalculates the longitudinal steel ratio which is used in the method "ConfinementRatio," then returns to the event handler the confinement ratio of the confined concrete column. The compression strain of the confined column is calculated using the "CompressiveStrainOfConfinedColumn" method. The concrete stress and steel stresses are calculated using the "ConcreteStress" and "SteelStress" methods, respectively. All these values are converted to strings and displayed in the window form. The allowable stress levels of the concrete, $65 \%$ of the compressive strength of concrete, and the steel, $60 \%$ of the yield strength of steel, are calculated and also displayed to the user.

All of these $\mathrm{ACl}$ requirements are then compared and informs the user of the outcome and appropriate checkboxes are updated to reflect the outcome. The confinement ratio is checked to see if it is greater than 0.08 as mentioned in $\mathrm{ACl} 440.2 \mathrm{R}-08$. The compressive strain has to be less than 0.01 as stated by Eq. 2.26. The concrete and steel stresses have to be less than the allowable stress levels as mentioned above. 
The last check the event handler makes is to see if all the checkboxes are true. When this occurs a message box pops up informing the user that the design met all requirements. Else, the message box says the design has failed requirements and needs to be redesigned.

\subsubsection{1 "frmBeamColumnProperties"}

The beam-column form for collecting all the required properties and values is the "frmBeamColumnProperties" form (Figure 3.14). Just like other properties forms, this form uses textboxes for entering values and arranges these items in different group boxes for each type of material or significant items. There is an additional group box for required loading which is for entering the factored moment and factored axial required loading for the beam-column. Since the beam-column path is not a design path, but rather a verification path, the application will not use the "frmLoading" form to determining the maximum loading combination. Instead, the user will need to determine this prior and input the values.

The geometric properties group box is identical to the same group box in the "frmColumnProperties" form, and uses the same event handlers to calculate the gross cross-sectional area depending on which radio button is selected. Please refer to Sections 4.3.9.1 through 4.3.9.5 for the process of these event handlers.

Since this path is only for verification, a textbox for the number of layers is added to the FRP properties group box because in order to verify a design is valid, the strengthening design must be included.

There is also a change in the concrete properties. Usually the compressive strength is an input value given by the user, but for beam-columns because of confined stress block parameters are dependent on the compressive strength, and the methods used for finding the stress block parameter 
are tailored to specific concrete compressive strengths, a drop down list is used to limit the user's selection. The drop down list has three strengths: $20.68 \mathrm{mPa}, 27.57 \mathrm{mPa}$, and $34.37 \mathrm{mPa}$.

The steel properties group box combines the textboxes and need values from beams in flexure and columns, but it also two unique list boxes which are connect to the distance of reinforcement and the area of steel textboxes. In order to develop the interaction diagram for a beam-column, individual layers of steel reinforcement must be known. So the textbox for distance is used for entering a layer's distance from the compression side of the concrete beam-column, while the textbox for area of steel is used to input the area of steel for the current layer of steel reinforcement. There are event handlers for these two textboxes to update the list boxes which show the list of distances and areas for each layer of steel entered (Figure 3.15).

\subsubsection{1 "frmBeamColumnProperties" event handler "txtDistanceOfReinforcement_LostFocus"}

So when the user takes the focus away from the distance of Reinforcement textbox, the event handler will validate the inputs for the distance and area of steel and then add them to the corresponding list boxes. First the upper and lower limits of the area of steel and distance are defined. Then the area of steel textbox is checked to see if it is not null or empty, there saying that the user has entered something in the textbox. Afterwards, using Figure 4.5 structure, the event handler validates the area of steel and the distance entered. If both are valid, the distance is added to the list array "distanceOfSteel" and the area is added to the list array "areaOfSteel." Then the text from the two textboxes is added to the items list of the list box to be displayed to the user in the windows form. Lastly, the textboxes of the distance and area are cleared so the next layer can be entered and processed (See Figure 3.15). 


\subsubsection{2 "frmBeamColumnProperties" event handler "txtAreaOfSteel_LostFocus"}

This event handler does the same thing as described in section 4.3.11.1 expect instead of checking to see if the textbox for the area of steel is null or empty, the event handler checks to the text box for the distance of steel to see if the null or empty. If not, the process is what is described in section

\subsubsection{1.}

\subsubsection{3 "frmBeamColumnProperties" event handler "btnContinue_Clicked"}

The purpose of this event handler is to validate all the inputs and push the values into the next form. The process is very similar to all other properties forms. For every textbox input, the structure from Figure 4.5 is used to validate. For every radio button, the process from Figure 4.6 is used.

The environmental factor is calculated using the "EnvironmentalFactor" method. Next the form "frmBeamColumnResults" is created and all the public variables are updated with the corresponding input values. Finally the new form is shown while the current form is hidden.

\subsubsection{2 "frmBeamColumnResults"}

This form's purpose is to generate the interaction diagram (Figure 3.16) to help verify a design for beam-columns that have been strengthened. The interaction diagram shows the relationship of the beam-column's capacity for moments and axial loads. The form shows a key in the top right corner of the diagram for the four different lines and the required loading point.

The window form has three buttons: Back, Close, and Show Results. The Back and Close buttons event handlers act as all other back and close buttons throughout the application. The Show Results button event handler generates the interaction diagram based on the inputs given in the "frmBeamColumnProperties" form. 


\subsubsection{1 "frmBeamColumnResults" event handler "btnShowResults_Click"}

The "btnShowResults_Click" event handler generates the interaction diagram for the beamcolumn by calculating six different values on the diagram, each representing a different failure mode, and then plotting them with respect to a moment-axial load coordinate system.

First the event handler defines all local variables, which include eight predefined arrays for each type of capacity which include strengthened, non-strengthened, factored, and non-factored moments and axial loads. These arrays will assign values as the points are calculated and eventually used to plot the interaction diagram.

Next, preliminary values that will be used throughout the plotting process are calculated. The eccentricity factor is set equal to 0.8 if the reinforcement is ties or equal to 0.85 for spiral reinforcement. The depth of the tensile steel is equal the difference between the height of the member and the clear cover. The ultimate tensile strength and rupture strain is calculated by using the "ultimateTensileStrengthOfFRP" and "UltimateRuptureStrainOfFRP" methods. The modulus of the FRP is then calculated using the "ModulusOfFRP" method. The concrete stress block factor is determined by using the "StressBlockFactor" method. The total area of steel is returned from the "TotalAreaOfSteel" method and the effective strain limit is determined by the "StrainEffective" method.

The confined concrete strength, the confined pressure and the axial strain of the confined concrete member are calculated using the "ConfinedPressure" and "UltimateAxialCompressionOfConfinedConcrete" methods. The "ConfinedConcreteCompressioveStrength" method is used to calculate the confined concrete strength.

With the concrete strength, confined concrete strength, and the compression strain, the stress block parameters A and B are calculated using the "StressBlockParameterA" and "StressBlockParameterB" methods to finish off all preliminary calculations. 
The first point to be calculated is Point A (See Figure 2.1) which represents the capacity of the member when it undergoes pure axial loading. This will be plotted on the axial capacity axis, and therefore, the all four moment arrays at index 0 , are set equal to 0 while "PointA" method is used to calculate the first values of the four axial arrays. It must be noted that in the "PointA" method, the input for the check integer changes with each array.

The check integer represents which array is being calculated because the process is slightly different for moment and axial loads when the member is factored and or strengthened. Therefore, 1 is for strengthened axial. 2 is for factored strengthened axial. 3 is for non-strengthened, non-factored axial. 4 is for factored non-strengthened. 5 is for strengthened moment. 6 is for factored strengthened moment. 7 is for non-strengthened, non-factored moment. 8 is for factored non-strengthened moment. Every time a point is calculated for all the arrays, the check integer will match the array so the method knows which point is being calculated.

The second point is Point $D$ (See Figure 2.1). Point $D$ determines the capacity of the beamcolumn by taking into account a load eccentricity due to imperfections in the construction or in the load estimates. Method "PointD" is used to calculate the value for index 1 in all the arrays.

Point B (See Figure 2.1) is when the strain distrubtion on the extreme compression fiber is equal to the ultimate concrete strain and the strain at the steel reinforcement closes to the tensile fiber is zero. Method "PointBorC" is used and the input for the ultimate concrete strain is 0.003 , and the ultimate strain of the steel is equal to 0 . Each array in index 2 is equal to Point $B$.

The next point, Point C (See Figure 2.1), assumes that strain distribution has the concrete crushing and the steel yielding simultaneously. Therefore the concrete strain is equal to 0.003 and the steel strain equal to the ultimate steel strain. The return value for Point C from "PointBorC" method is set to index 3 for every array. 
Point $E$ (See Figure 2.1) uses a fixed value of eccentricity. This means that the interaction diagram uses a value of eccentricity that produces a 45 degree line that intersects the diagram. Method "PointE" is used to accomplish this calculation and the value is place in index 4 of all the arrays.

The Last point, Point $F$ (See Figure 2.1), is when the beam-column is experiencing pure flexure. Therefore the strength reduction factor is set to 0.9 , and all the axial loading arrays are equal to zero for plotting purposes. Also, the interaction diagram for pure flexure does not consider FRP strengthening according to $\mathrm{ACl}$, thus only unconfined capacity is calculated using "PointF" method. The return value is set to index 5 of the moment arrays, even the strengthened arrays so they can be plotted.

After all six points are calculated for every array, the event handler adds the required moment and axial capacity to the chart. Next Point $C$ and $E$ are compared to each other because if the greater axial capacity of the two needs to be plotted after the smaller one. So if Point C is less than Point E, every value at index 3 and 4 are reversed in all arrays.

Finally, the interaction diagram is plotted using four for-loops: strengthened, factored strengthened, non-strengthened, and non-strengthened and non-factored. The for-loop uses the counter integer " $\mathrm{i}$ " and sets it equal to 0 so the loop starts with the first index of the array. The loop than adds a point to corresponding series to the chart for the index "i." The for-loop will increase the value of "i" by 1 until " $\mathrm{i}$ " is equal to or greater than the length of the array which is six. This allows for each item in the array to be plotted. 


\subsection{Traceability Matrix}

The traceability matrix allows a quick glance on which forms and classes are correlated and how dependent some classes are compared to others.

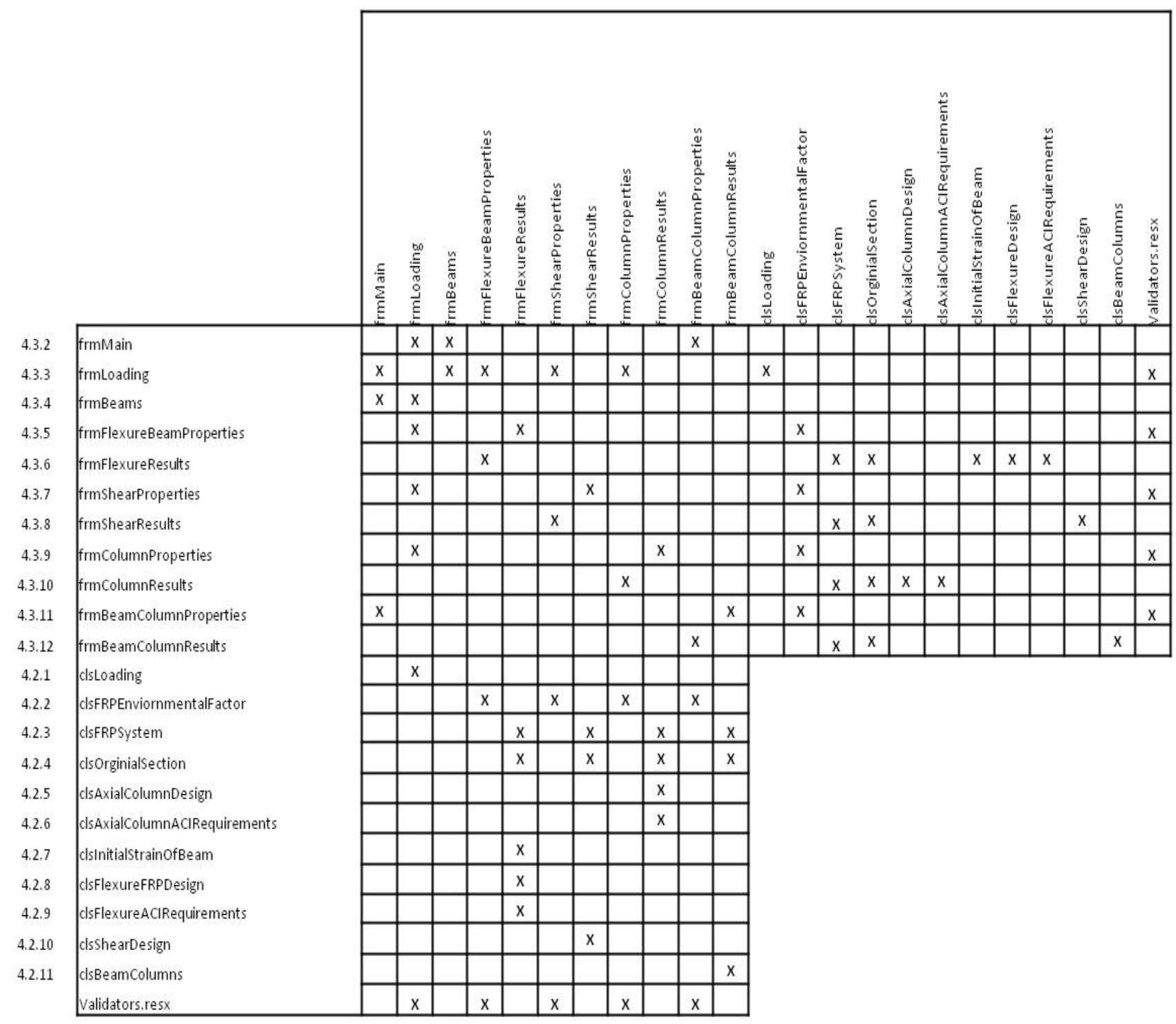

Figure 4.6 Traceability Matrix 


\section{Chapter 5 - Software Output}

\subsection{Introduction}

In order to validate the developed methods, a comparison of outputs of the application versus examples is shown in this chapter to demonstrate the use of the application and consistency among answers. Examples were taken from a variety of sources and updated to match the $\mathrm{ACl} 440.2 \mathrm{R}-08$. Each comparison will show the required inputs for the design process and the forms used to enter these values and to display the results. Also, the updated computations for the examples will be discussed, and details of manual calculations of every example can be seen in the associated appendix.

\subsection{Strengthening Beams in Flexure}

\subsubsection{Example A}

Example $A$ is a rectangular reinforced concrete beam experiencing an additional $60 \%$ of the current live loading, and strengthening is required [4]. Table 5.1 shows the input values needed to complete the design process which includes geometric, steel, concrete, and FRP properties. Figure 5.1 displays these values within the appropriate form.

Table 5.1 Example A Inputs

\begin{tabular}{|c|c|c|c|c|c|}
\hline \multicolumn{2}{|c|}{ Geometric Properties [4] } & \multicolumn{2}{|c|}{ Steel Properties [4] } & \multicolumn{2}{|l|}{ FRP Properties [4] } \\
\hline length [mm] & 6300 & Area of steel [mm^2] & 850 & FRP tensile strength [mPa] & 2200 \\
\hline base $[\mathrm{mm}]$ & 300 & yield strength [mPa] & 413.68 & FRP ultimate strain & 0.011 \\
\hline height [mm] & 600 & $\begin{array}{c}\text { modulus of steel } \\
{[\mathrm{mPa}]}\end{array}$ & 200000 & ply thickness [mm] & 0.333 \\
\hline $\begin{array}{c}\text { Moment of } \\
\left.\text { Inertia } \mathrm{mm}^{\wedge} 4\right]\end{array}$ & $5.40 \mathrm{E}+09$ & clear cover $[\mathrm{mm}]$ & 40 & Fiber & Carbon \\
\hline \multicolumn{2}{|c|}{ Loading Requirements [4] } & \multicolumn{2}{|c|}{ Exposure } & \multicolumn{2}{|c|}{ Concrete Properties [4] } \\
\hline $\begin{array}{c}\text { Dead Load [N- } \\
\mathrm{mm}]\end{array}$ & $6.7 \mathrm{E}+07$ & \multicolumn{2}{|c|}{ Interior } & Concrete Strength [mPa] & 34.5 \\
\hline $\begin{array}{l}\text { Live Load [N- } \\
\mathrm{mm}]\end{array}$ & $8.34 \mathrm{E}+07$ & & & & \\
\hline
\end{tabular}




\begin{tabular}{|c|c|c|}
\hline \multicolumn{2}{|l|}{ Loading Conditions } & - $\square \mathrm{x}$ \\
\hline \multicolumn{3}{|c|}{$\begin{array}{l}\text { Please Input unfactored Loading Requirements } \\
\text { for the critical section of the member: }\end{array}$} \\
\hline Dead Load: & 66980000 & $\mathrm{~N}-\mathrm{mm}$ \\
\hline Live Load: & 83350000 & $\mathrm{~N}-\mathrm{mm}$ \\
\hline $\begin{array}{l}\text { Lateral Fluid } \\
\text { Pressure Load: }\end{array}$ & 0 & $\mathrm{~N}-\mathrm{mm}$ \\
\hline Snow Load: & 0 & $\mathrm{~N}-\mathrm{mm}$ \\
\hline Rain Load: & 0 & $\mathrm{~N} \cdot \mathrm{mm}$ \\
\hline Wind Load: & 0 & $\mathrm{~N} \cdot \mathrm{mm}$ \\
\hline Earthquake Load: & 0 & $\mathrm{~N}-\mathrm{mm}$ \\
\hline $\begin{array}{l}\text { Temperature, } \\
\text { Creep, } \\
\text { Shrinkage load: }\end{array}$ & 0 & $\mathrm{~N}-\mathrm{mm}$ \\
\hline Soil Load: & 0 & $\mathrm{~N}-\mathrm{mm}$ \\
\hline Live Roof Load: & 0 & $\mathrm{~N} \cdot \mathrm{mm}$ \\
\hline Back & & Continue \\
\hline
\end{tabular}

\begin{tabular}{|c|c|c|}
\hline \multicolumn{3}{|l|}{ Beam Properties } \\
\hline \multicolumn{3}{|l|}{ Geometric Properties } \\
\hline Length of Beam: & 6300 & $\mathrm{~mm}$ \\
\hline Base of Beam: & 300 & $\mathrm{~mm}$ \\
\hline Height of Beam & 600 & $\mathrm{~mm}$ \\
\hline $\begin{array}{l}\text { Moment of inertia } \\
\text { before cracking: }\end{array}$ & 5400000000 & $\mathrm{~mm}^{\wedge} 4$ \\
\hline \multicolumn{3}{|l|}{ FRP Properties } \\
\hline $\begin{array}{l}\text { Tensile Strength } \\
\text { of FRP: }\end{array}$ & 2200 & $\mathrm{MPa}$ \\
\hline Ply thickness: & .333 & $\mathrm{~mm}$ \\
\hline Ultimate Strain: & .011 & \\
\hline \multicolumn{3}{|l|}{ Select Fiber Type: } \\
\hline (- Carbon $\mathrm{C}$ & Glass $C \mathrm{Ar}$ & \\
\hline
\end{tabular}

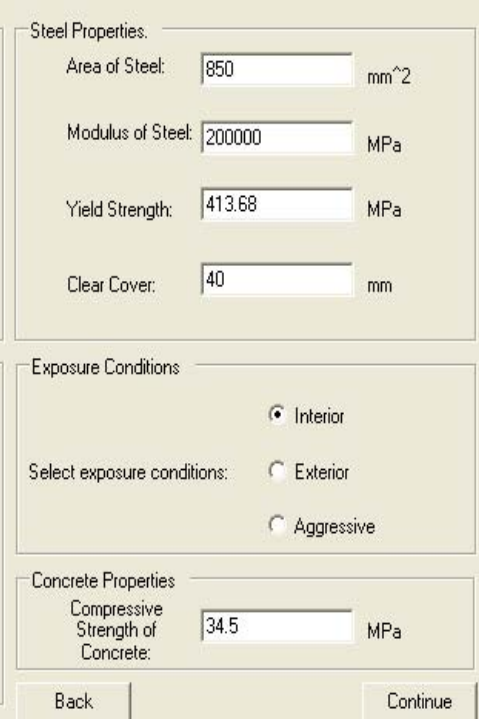

Figure 5.1 Example A: Windows Application Inputs

Flexure Design and Results

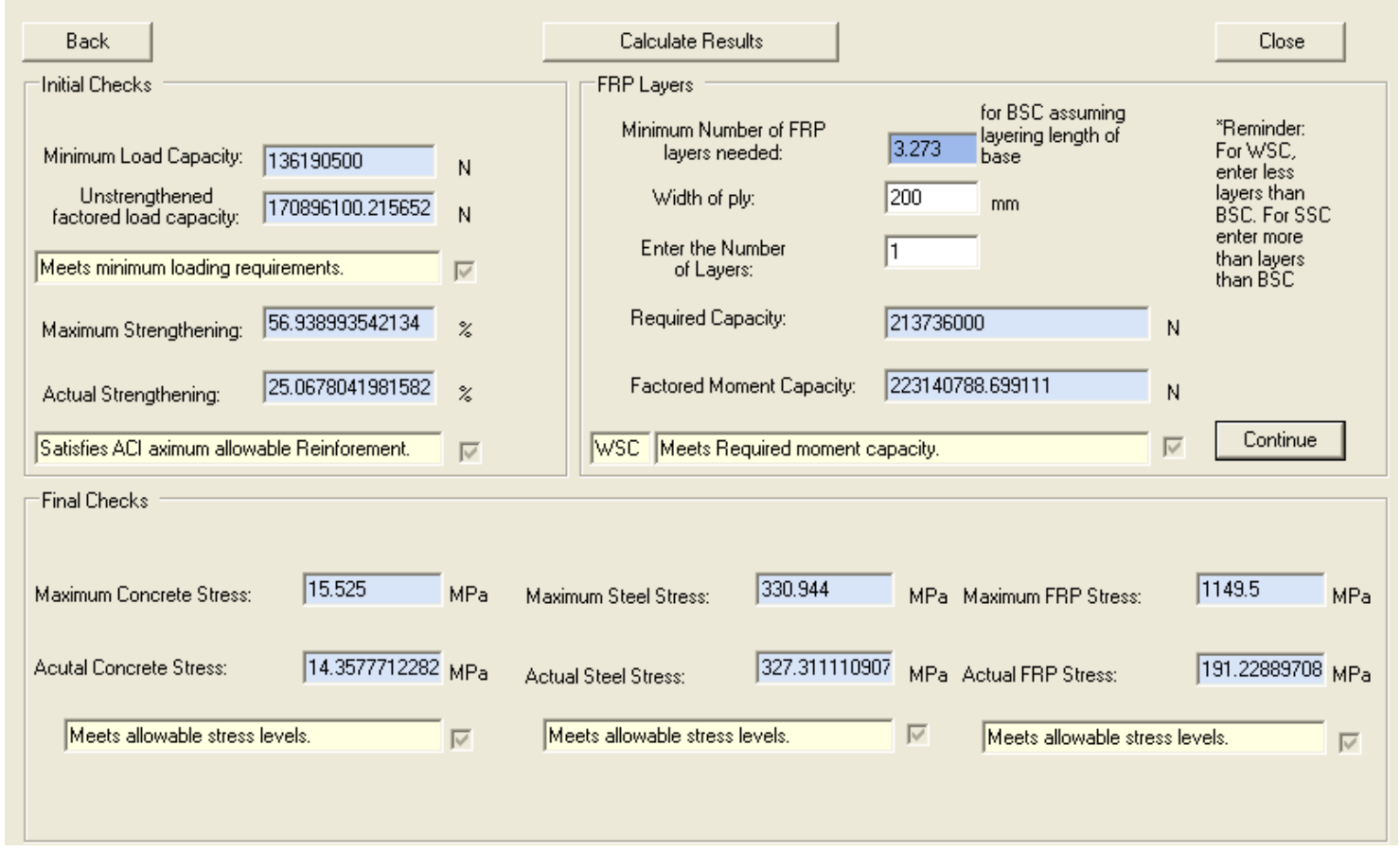

Figure 5.2 Example A Outputs 
After running the values through the application, the minimum number of layers needed to achieve balanced strengthening configuration was 3.273 layers. The updated example solved by hand also produced a value of 3.273 layers. One layer with a width of $200 \mathrm{~mm}$ was selected to continue the design process and check all requirements. A comparison of the results is shown in Table. 5.2. This example is updated with respect to [4] to meet current ACl 440.2R-08 pratices. See Appendix A for hand calculations.

Table 5.2 Comparison of Example A outputs

\begin{tabular}{|l|c|c|c|}
\cline { 2 - 4 } \multicolumn{1}{c|}{} & $\begin{array}{c}\text { Updated } \\
\text { Example }\end{array}$ & Application & Difference \\
\hline Required capacity & $2.14 \mathrm{E}+08$ & $2.14 \mathrm{E}+08$ & $0.00 \mathrm{E}+00$ \\
\hline Factored capacity & $2.23 \mathrm{E}+08$ & $2.23 \mathrm{E}+08$ & $0.00 \mathrm{E}+00$ \\
\hline $\begin{array}{l}\text { actual concrete } \\
\text { stress }\end{array}$ & 14.3577 & 14.357 & $7.00 \mathrm{E}-04$ \\
\hline actual steel stress & 327.311 & 327.302 & $9.00 \mathrm{E}-03$ \\
\hline actual FRP stress & 191.229 & 191.227 & $2.00 \mathrm{E}-03$ \\
\hline
\end{tabular}

\subsubsection{Example B}

Example $B$ is a rectangular beam requiring strengthening to resisted the additional loading [6].

Table 5.3 provides the inputs from the updated example and will be used in the application. Figure 5.3 demonstrates these values in the window forms. This example is updated with respect to [6] to meet current $\mathrm{ACl} 440.2 \mathrm{R}-08$ pratices and by increasing the required loading. See Appendix $\mathrm{A}$ for hand calculations. 
Table 5.3 Example B Inputs

\begin{tabular}{|c|c|c|c|c|c|}
\hline \multicolumn{2}{|c|}{ Geometric Properties [6] } & \multicolumn{2}{|c|}{ Steel Properties [6] } & \multirow{2}{*}{$\begin{array}{c}\text { FRP Properties [6] } \\
\text { FRP tensile strength [mPa] }\end{array}$} & \multirow[b]{2}{*}{1020} \\
\hline length [mm] & 9140 & Area of steel $\left[\mathrm{mm}^{\wedge} 2\right]$ & 2458 & & \\
\hline base $[\mathrm{mm}]$ & 305 & yield strength [mPa] & 414 & FRP ultimate strain & 0.0129 \\
\hline height $[\mathrm{mm}]$ & 660 & modulus of steel [mPa] & 200000 & ply thickness [mm] & 0.144 \\
\hline Moment of Inertia $\left.\mathrm{mm}^{\wedge} 4\right]$ & $7.31 \mathrm{E}+09$ & clear cover [mm] & 77 & Fiber & Glass \\
\hline \multicolumn{2}{|c|}{ Loading Requirements [6] } & \multicolumn{2}{|l|}{ Exposure } & \multicolumn{2}{|l|}{ Concrete Properties [6] } \\
\hline Dead Load [N-mm] & $3.78 \mathrm{E}+08$ & \multicolumn{2}{|l|}{ Exterior } & Concrete Strength [mPa] & 27.6 \\
\hline Live Load [N-mm] & $4.95 E+08$ & & & & \\
\hline
\end{tabular}

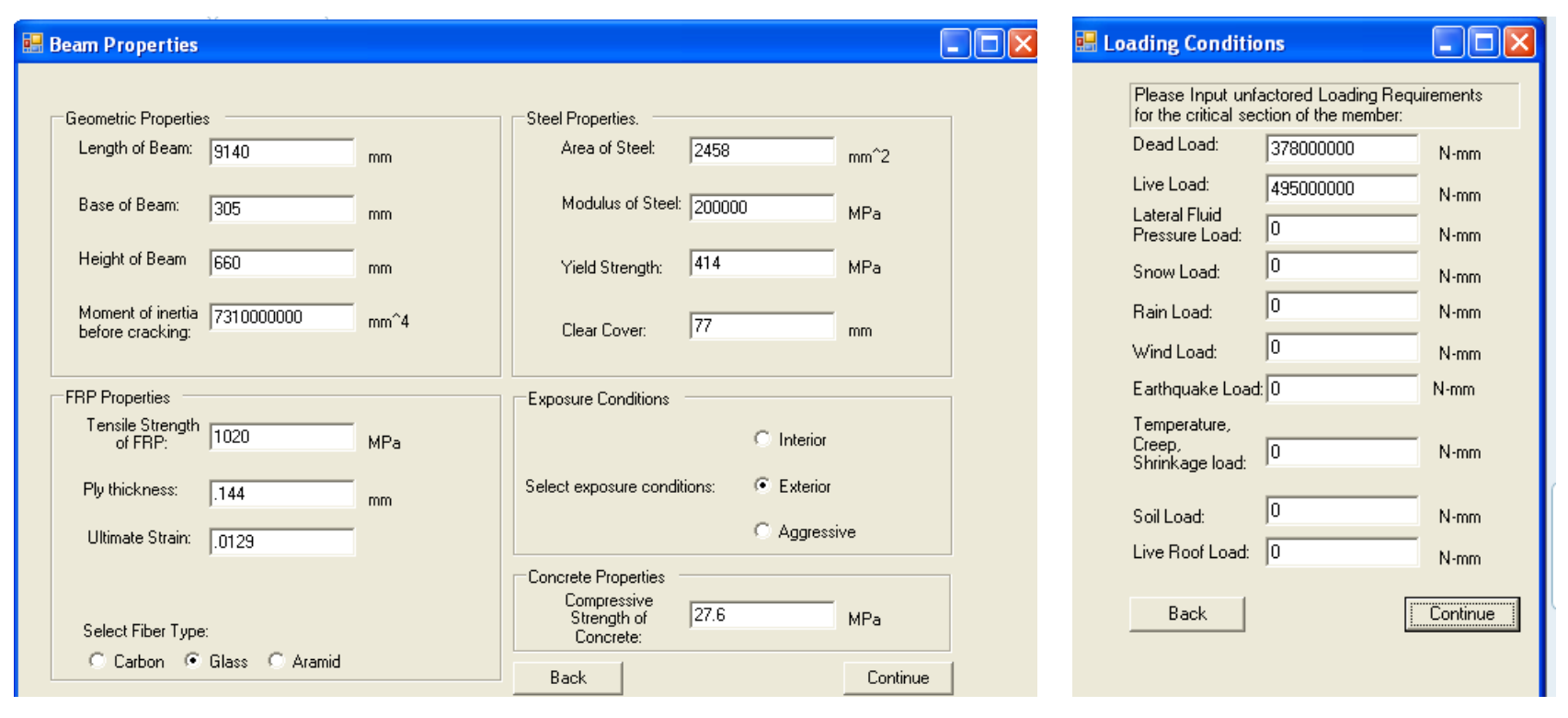

Figure 5.3 Example: B Window Form Inputs 


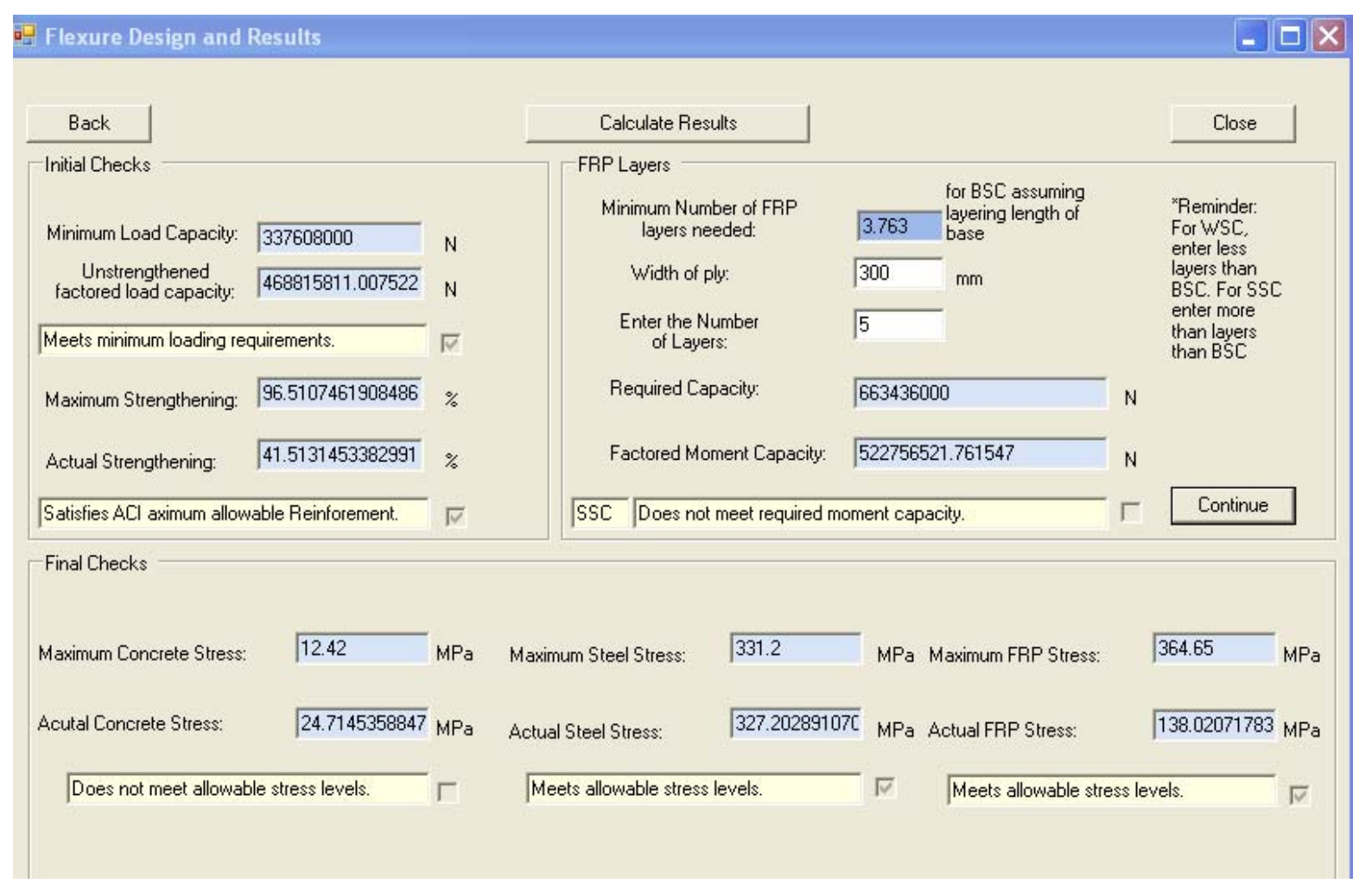

\section{Figure 5.4 Example B Outputs}

The application suggests 3.763 layers to obtain balanced strengthening configuration while the updated Example B suggest 3. 774. Five layers of FRP reinforcement was chosen to be applied with a width of $300 \mathrm{~mm}$. Table 5.4 shows calculated values for capacities and all required checks. Both the application and the example have the beam not meeting the required capacity nor the maximum allowable concrete stress.

\section{Table 5.4 Comparison of Example B Outputs}

\begin{tabular}{|l|c|c|c|}
\cline { 2 - 4 } \multicolumn{1}{c|}{} & $\begin{array}{c}\text { Updated } \\
\text { Example }\end{array}$ & Application & Difference \\
\hline Required capacity & $6.63 \mathrm{E}+08$ & $6.63 \mathrm{E}+08$ & $0.00 \mathrm{E}+00$ \\
\hline Factored capacity & $5.23 \mathrm{E}+08$ & $5.23 \mathrm{E}+08$ & $0.00 \mathrm{E}+00$ \\
\hline $\begin{array}{l}\text { actual concrete } \\
\text { stress }\end{array}$ & 24.714 & 24.714 & $0.00 \mathrm{E}+00$ \\
\hline actual steel stress & 327.198 & 327.203 & $-5.00 \mathrm{E}-03$ \\
\hline actual FRP stress & 138.019 & 138.021 & $-2.00 \mathrm{E}-03$ \\
\hline
\end{tabular}




\subsubsection{Example C}

Example $\mathrm{C}$ is an updated example of rectangular beam experiencing an increase of $50 \%$ of the current live loading [3]. Table 5.5 shows the parameters of the example which are entered into the application. Figure 5.5 and 5.6 shows the values in the application and the outputs. This example is updated with respect to [3] to meet current $A C l$ 440.2R-08 pratices. See Appendix A for hand calculations.

\section{Table 5.5 Example C Inputs}

\begin{tabular}{|c|c|c|c|c|c|}
\hline \multicolumn{2}{|c|}{ Geometric Properties [3] } & \multicolumn{2}{|c|}{ Steel Properties [3] } & \multicolumn{2}{|c|}{ FRP Properties [3] } \\
\hline length [mm] & 7315.2 & Area of steel [mm^2] & 1935.6 & FRP tensile strength [mPa] & 620.55 \\
\hline base $[\mathrm{mm}]$ & 305 & yield strength [mPa] & 414 & FRP ultimate strain & 0.017 \\
\hline height [mm] & 610 & modulus of steel [mPa] & 200000 & ply thickness [mm] & 1 \\
\hline $\begin{array}{c}\text { Moment of Inertia } \\
\left.\mathrm{mm}^{\wedge} 4\right]\end{array}$ & $5.77 E+09$ & clear cover $[\mathrm{mm}]$ & 64 & Fiber & Carbon \\
\hline \multicolumn{2}{|c|}{ Loading Requirements [3] } & \multicolumn{2}{|c|}{ Exposure } & \multicolumn{2}{|l|}{ Concrete Properties [3] } \\
\hline Dead Load [N-mm] & $1.00 \mathrm{E}+08$ & \multicolumn{2}{|l|}{ interior } & Concrete Strength [mPa] & 34.5 \\
\hline Live Load [N-mm] & $1.76 \mathrm{E}+08$ & & & & \\
\hline
\end{tabular}

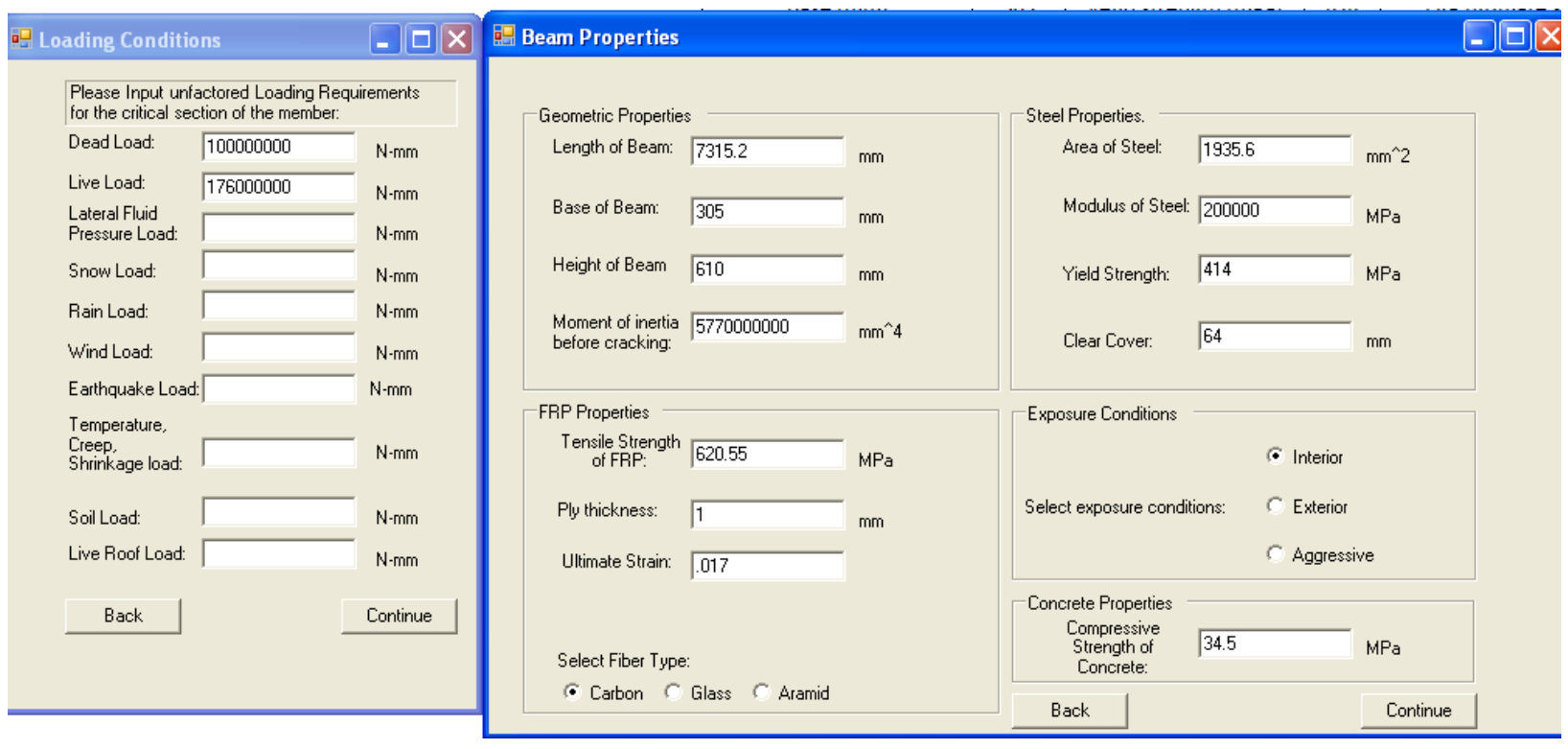

Figure 5.5 Example C: Windows Forms Inputs 


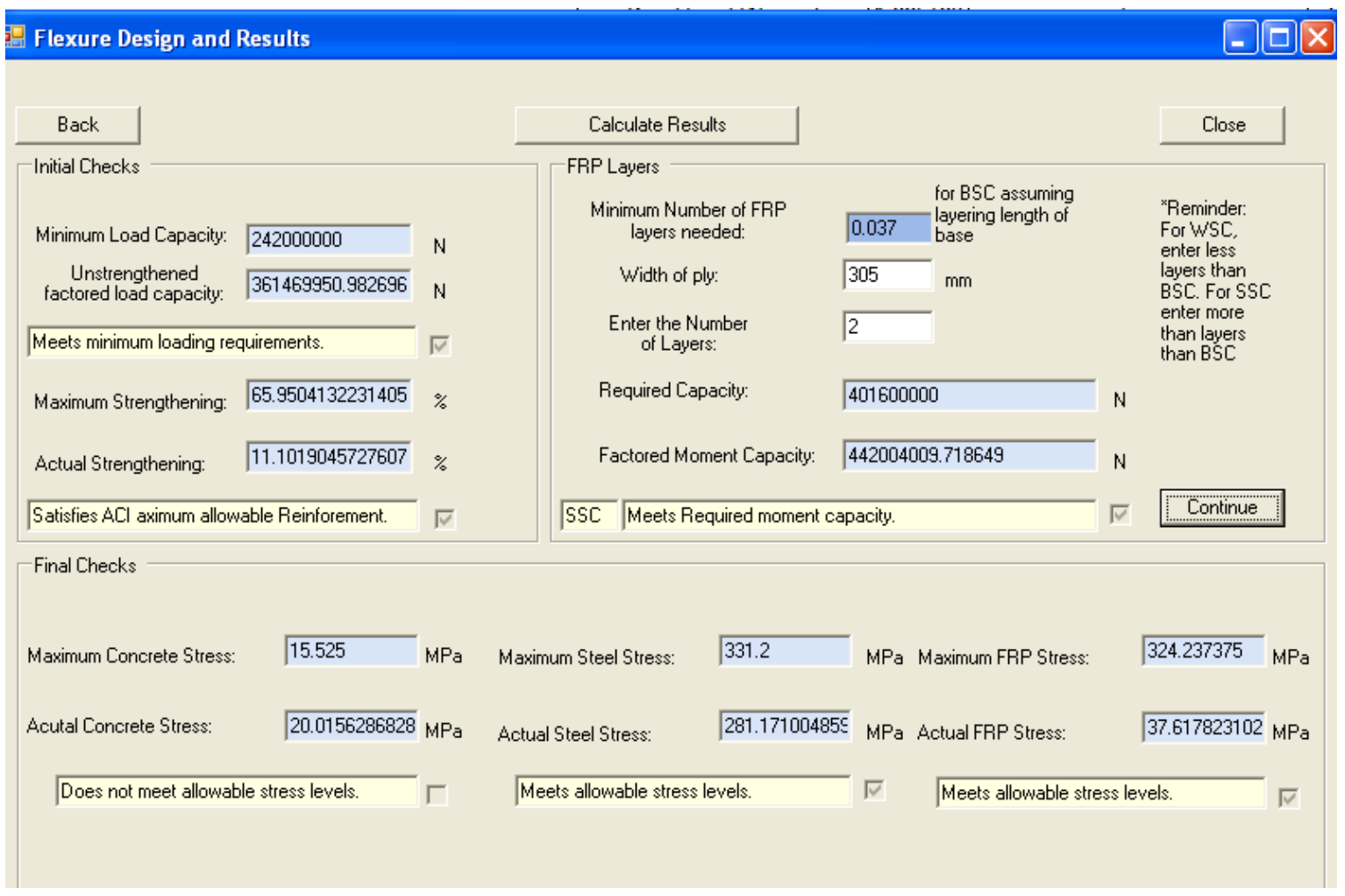

\section{Figure 5.6 Example C Outputs}

The application suggests using only 0.037 layers to meet BSC while the updated example also gives a suggestion of 0.037 . Two layers of FRP reinforcement was selected with a width of $305 \mathrm{~mm}$. Both the application and the example says the design does not meet the maximum allowable stress levels. A comparison of the application outputs and the example can be seen in Table. 5.6.

\section{Table 5.6 Comparison of Example C Outputs}

\begin{tabular}{|l|c|c|c|}
\cline { 2 - 4 } \multicolumn{1}{c|}{} & $\begin{array}{c}\text { Updated } \\
\text { Example }\end{array}$ & Application & Difference \\
\hline Required capacity & $4.01 \mathrm{E}+08$ & $4.02 \mathrm{E}+08$ & $-1.00 \mathrm{E}+06$ \\
\hline Factored capacity & $4.42 \mathrm{E}+08$ & $4.42 \mathrm{E}+08$ & $0.00 \mathrm{E}+00$ \\
\hline $\begin{array}{l}\text { actual concrete } \\
\text { stress }\end{array}$ & 19.995 & 20.016 & $-2.10 \mathrm{E}-02$ \\
\hline actual steel stress & 280.89 & 281.171 & $-2.81 \mathrm{E}-01$ \\
\hline actual FRP stress & 37.551 & 37.618 & $-6.70 \mathrm{E}-02$ \\
\hline
\end{tabular}




\subsubsection{Example D}

Example $D$ is a reinforced concrete rectangular beam that needs addition of strength because the current level of live load was doubled [5]. Table 5.7 shows the loading and properties of the beam and FRP reinforcement. Figure 5.7 and 5.8 displays these values in the application as well as the outputs of the design. This example is updated with respect to [5] to meet current $\mathrm{ACl} 440.2 \mathrm{R}-08$ pratices. See Appendix A for hand calculations.

Table 5.7 Example D Inputs

\begin{tabular}{|c|c|c|c|c|c|}
\hline \multicolumn{2}{|c|}{ Geometric Properties [5] } & \multicolumn{2}{|c|}{ Steel Properties [5] } & \multicolumn{2}{|l|}{ FRP Properties [5] } \\
\hline length [mm] & 6700 & Area of steel [mm^2] & 2026.8 & FRP tensile strength [mPa] & 650 \\
\hline base $[\mathrm{mm}]$ & 381 & yield strength [mPa] & 414 & FRP ultimate strain & 0.017 \\
\hline height [mm] & 610 & modulus of steel [mPa] & 200000 & ply thickness [mm] & 0.89 \\
\hline Moment of Inertia $\mathrm{mm}^{\wedge} 4$ ] & $7.21 \mathrm{E}+09$ & clear cover [mm] & 64 & Fiber & Carbon \\
\hline \multicolumn{2}{|c|}{ Loading Requirements [5] } & \multicolumn{2}{|c|}{ Exposure } & \multicolumn{2}{|l|}{ Concrete Properties [5] } \\
\hline Dead Load [N-mm] & $9.01 \mathrm{E}+07$ & \multicolumn{2}{|l|}{ interior } & Concrete Strength [mPa] & 38 \\
\hline Live Load [N-mm] & $2.05 \mathrm{E}+08$ & & & & \\
\hline
\end{tabular}

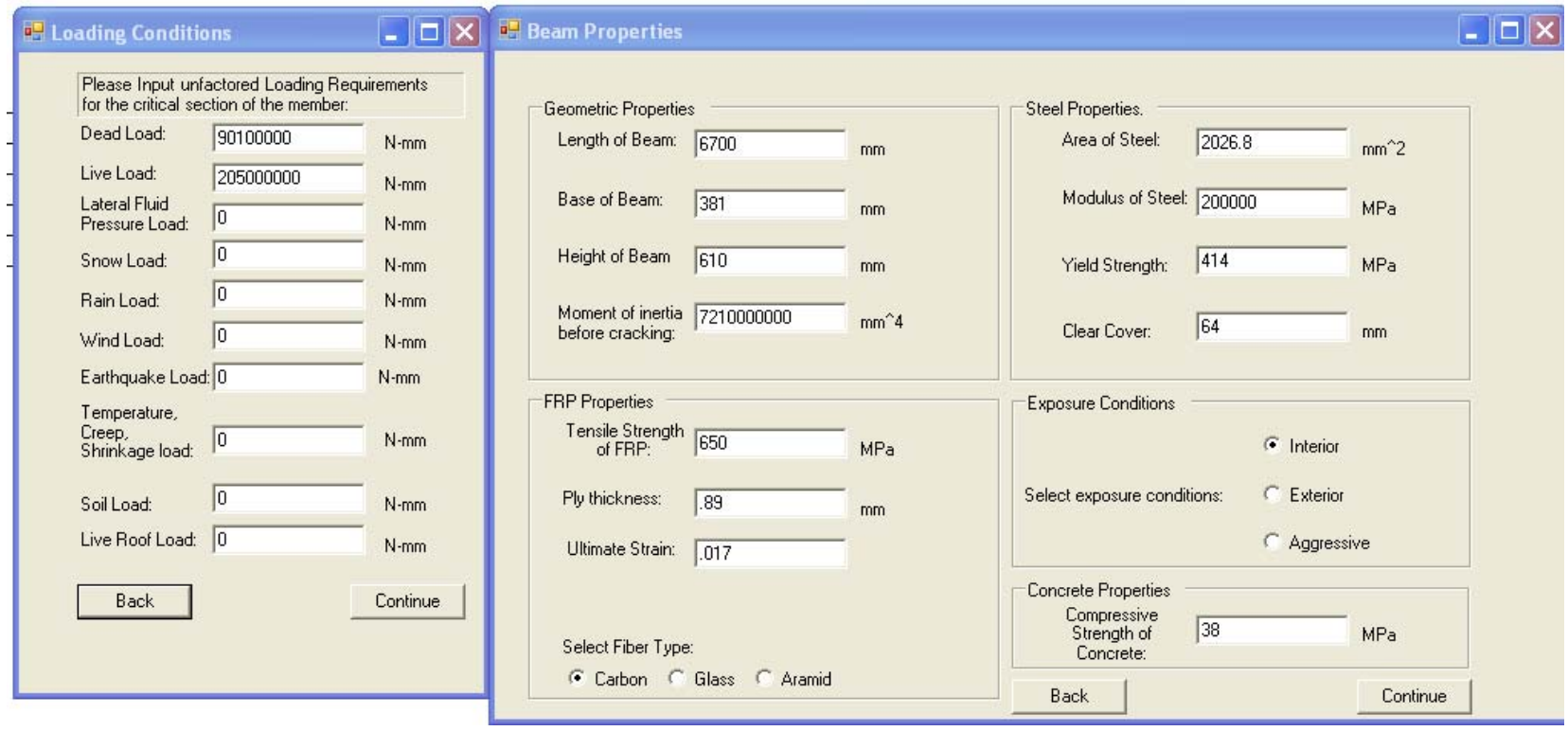

Figure 5.7 Example D: Windows Form Inputs 


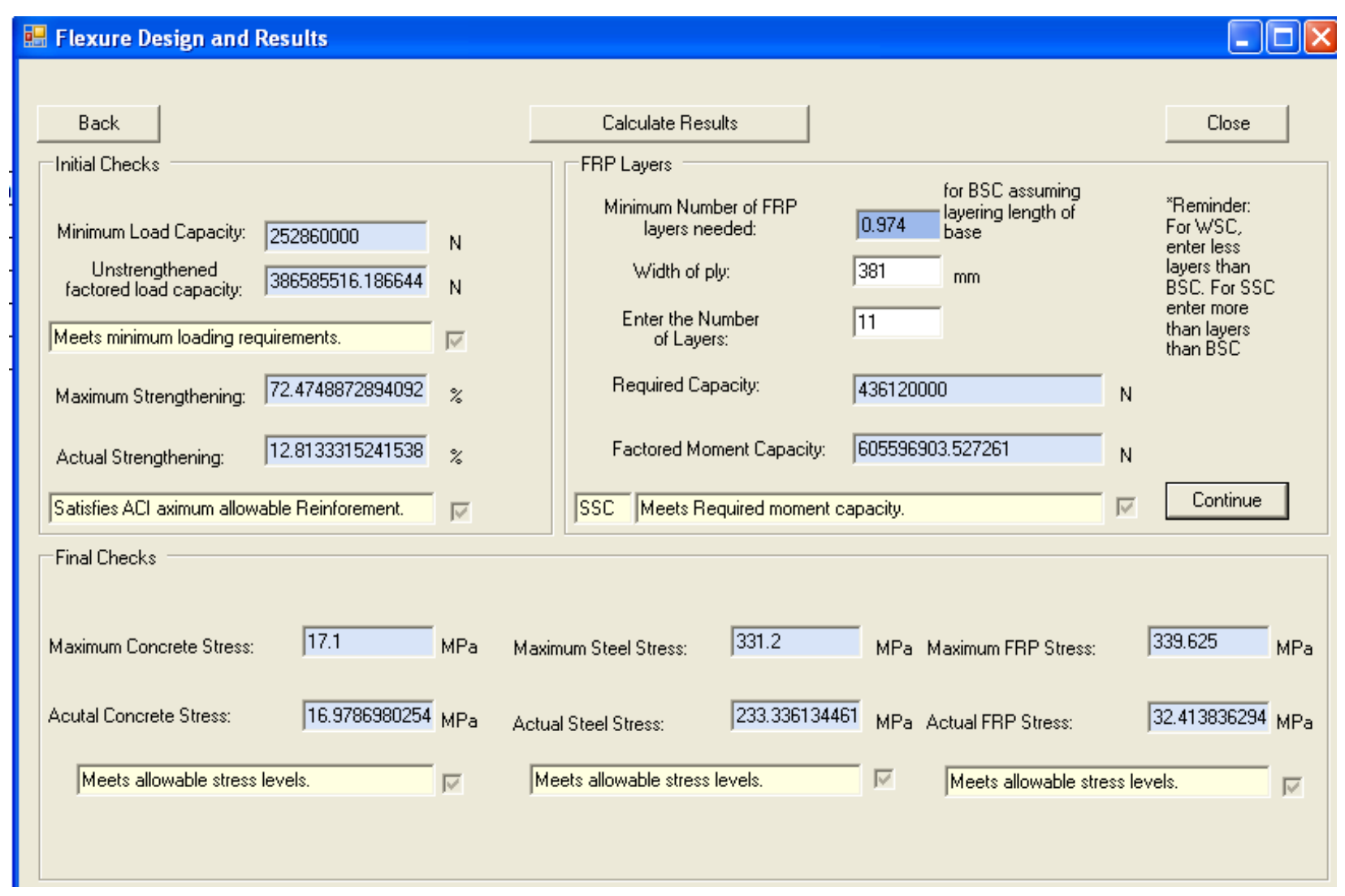

Figure 5.8 Example D Outputs

Both the application and the example suggest for 0.974 layers of FRP to reach BSC. To demonstrate the application can handle larger number of layers than a few, 11 layers with a width of $381 \mathrm{~mm}$ were chosen. All requirements were met by the application and the updated example. Table 5.8 compares the output values.

\section{Table 5.8 Comparison of Example D Outputs}

\begin{tabular}{|l|c|c|c|}
\cline { 2 - 4 } \multicolumn{1}{c|}{} & $\begin{array}{c}\text { Updated } \\
\text { Example }\end{array}$ & Application & Difference \\
\hline Required capacity & $4.36 \mathrm{E}+08$ & $4.36 \mathrm{E}+08$ & $0.00 \mathrm{E}+00$ \\
\hline Factored capacity & $6.06 \mathrm{E}+08$ & $6.06 \mathrm{E}+08$ & $0.00 \mathrm{E}+00$ \\
\hline $\begin{array}{l}\text { actual concrete } \\
\text { stress }\end{array}$ & 16.96 & 16.98 & $-2.00 \mathrm{E}-02$ \\
\hline actual steel stress & 233.084 & 233.336 & $-2.52 \mathrm{E}-01$ \\
\hline actual FRP stress & 32.366 & 32.413 & $-4.70 \mathrm{E}-02$ \\
\hline
\end{tabular}




\subsection{Strengthening Beams in Shear}

\subsubsection{Example E}

Example $\mathrm{E}$ is a beam experiencing an increase shearing live load by 50\% [4]. The parameters are listed in Table 5.9. Figures 5.9 and 5.10 show these values in the form application and the outputs generated. This example is updated with respect to [4] to meet current $\mathrm{ACl} 440.2 \mathrm{R}-08$ pratices. See Appendix A for hand calculations.

\section{Table 5.9 Example E Inputs}

\begin{tabular}{|c|c|c|c|c|c|}
\hline \multicolumn{2}{|c|}{ Geometric Properties [4] } & \multicolumn{2}{|c|}{ Steel Properties [4] } & \multicolumn{2}{|l|}{ FRP Properties [4] } \\
\hline length [mm] & 6300 & Area of steel [mm^2] & 141.7 & FRP tensile strength [mPa] & 3700 \\
\hline base $[\mathrm{mm}]$ & 300 & Shear spacing $[\mathrm{mm}]$ & 200 & FRP ultimate strain & 0.016 \\
\hline height [mm] & 500 & yield strength [mPa] & 275.79 & ply thickness [mm] & 0.2 \\
\hline Moment of Inertia $\mathrm{mm}^{\wedge} 4$ ] & $3.13 E+09$ & modulus of steel [mPa] & 200000 & Fiber & Carbon \\
\hline \multicolumn{2}{|c|}{ Loading Requirements [4] } & clear cover [mm] & 40 & \multicolumn{2}{|l|}{ Concrete Properties [4] } \\
\hline Dead Load [N-mm] & $7.25 \mathrm{E}+04$ & \multicolumn{2}{|l|}{ Exposure } & Concrete Strength [mPa] & 20.68 \\
\hline Live Load [N-mm] & $5.67 \mathrm{E}+04$ & \multicolumn{2}{|l|}{ interior } & & \\
\hline
\end{tabular}

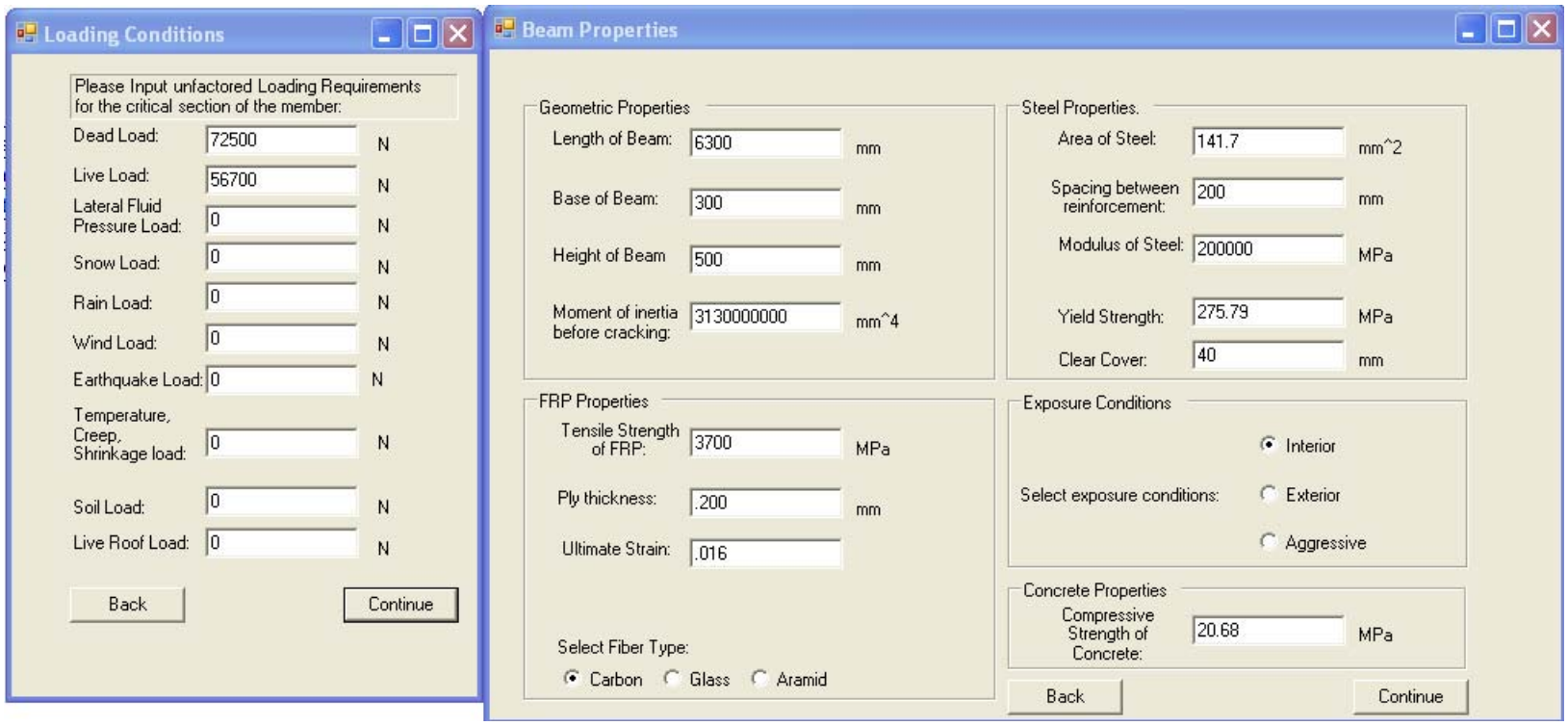

Figure 5.9 Example E: Windows Form Inputs 


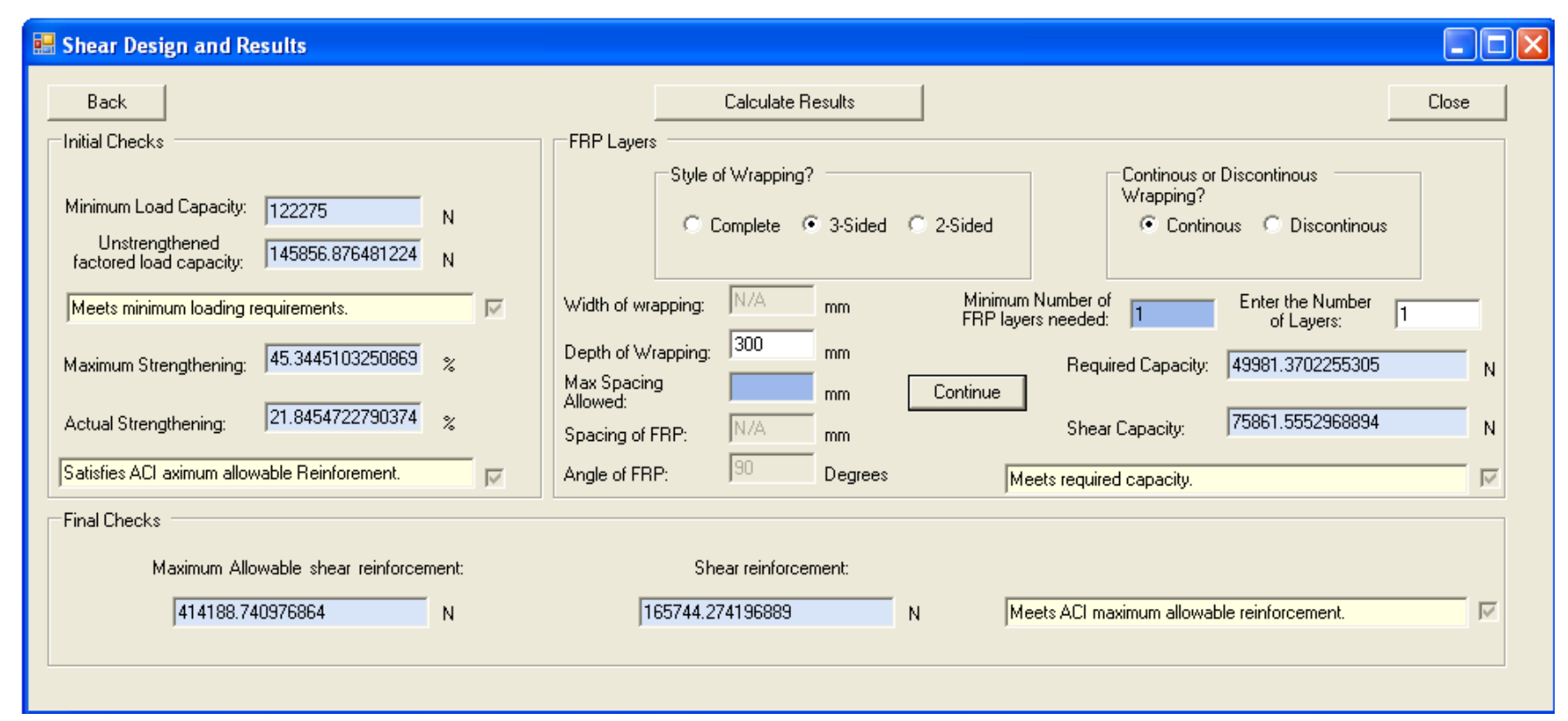

Figure 5.10 Example E Outputs

The design was decided to be 3-sided continuous wrapping of the beam. Therefore, the depth of the FRP to be applied to the side faces of the beam was selected to be $300 \mathrm{~mm}$. The application and the updated example both suggested 1 layer of FRP to meet the required shear capacity. Table 5.10 compares the example's capacities and allowable shear reinforcement to the application's outputs.

\section{Table 5.10 Comparison of Example E Outputs}

\begin{tabular}{|l|c|c|c|}
\cline { 2 - 4 } \multicolumn{1}{c|}{} & $\begin{array}{c}\text { Updated } \\
\text { Example }\end{array}$ & Application & Difference \\
\hline Required capacity & $4.99 \mathrm{E}+04$ & $5.00 \mathrm{E}+04$ & $-1.00 \mathrm{E}+02$ \\
\hline Factored capacity & $7.59 \mathrm{E}+04$ & $7.59 \mathrm{E}+04$ & $0.00 \mathrm{E}+00$ \\
\hline actual reinforcement & $1.66 \mathrm{E}+05$ & $1.66 \mathrm{E}+05$ & $0.00 \mathrm{E}+00$ \\
\hline
\end{tabular}

\subsubsection{Example $F$}

Example $\mathrm{F}$ is a rectangular concrete beam needing shear strengthening [6]. The loading requirements and all properties of the beam are located in Table. 5.11. Figures 5.11 and 5.12 show these properties in the window forms application and outcome of the parameters. This example is updated 
with respect to [6] to meet current $\mathrm{ACl} 440.2 \mathrm{R}-08$ practices and the required loading was increased. See Appendix A for hand calculations.

\section{Table 5.11 Example F Inputs}

\begin{tabular}{|c|c|c|c|c|c|}
\hline \multicolumn{2}{|c|}{ Geometric Properties [6] } & \multicolumn{2}{|c|}{ Steel Properties [6] } & \multicolumn{2}{|l|}{ FRP Properties [6] } \\
\hline length [mm] & 7620 & Area of steel [mm^2] & 258 & FRP tensile strength [mPa] & 3481.9 \\
\hline base $[\mathrm{mm}]$ & 356 & Shear spacing $[\mathrm{mm}]$ & 355.6 & FRP ultimate strain & 0.0153 \\
\hline height [mm] & 762 & yield strength [mPa] & 414 & ply thickness [mm] & 0.254 \\
\hline Moment of Inertia $\mathrm{mm}^{\wedge} 4$ ] & $1.31 \mathrm{E}+10$ & modulus of steel [mPa] & 200000 & Fiber & Carbon \\
\hline \multicolumn{2}{|c|}{ Loading Requirements [6] } & clear cover [mm] & 40 & \multicolumn{2}{|l|}{ Concrete Properties [6] } \\
\hline Dead Load [N-mm] & $2.44 \mathrm{E}+04$ & \multicolumn{2}{|l|}{ Exposure } & Concrete Strength [mPa] & 27.6 \\
\hline Live Load [N-mm] & $3.81 E+05$ & \multicolumn{2}{|l|}{ interior } & & \\
\hline
\end{tabular}

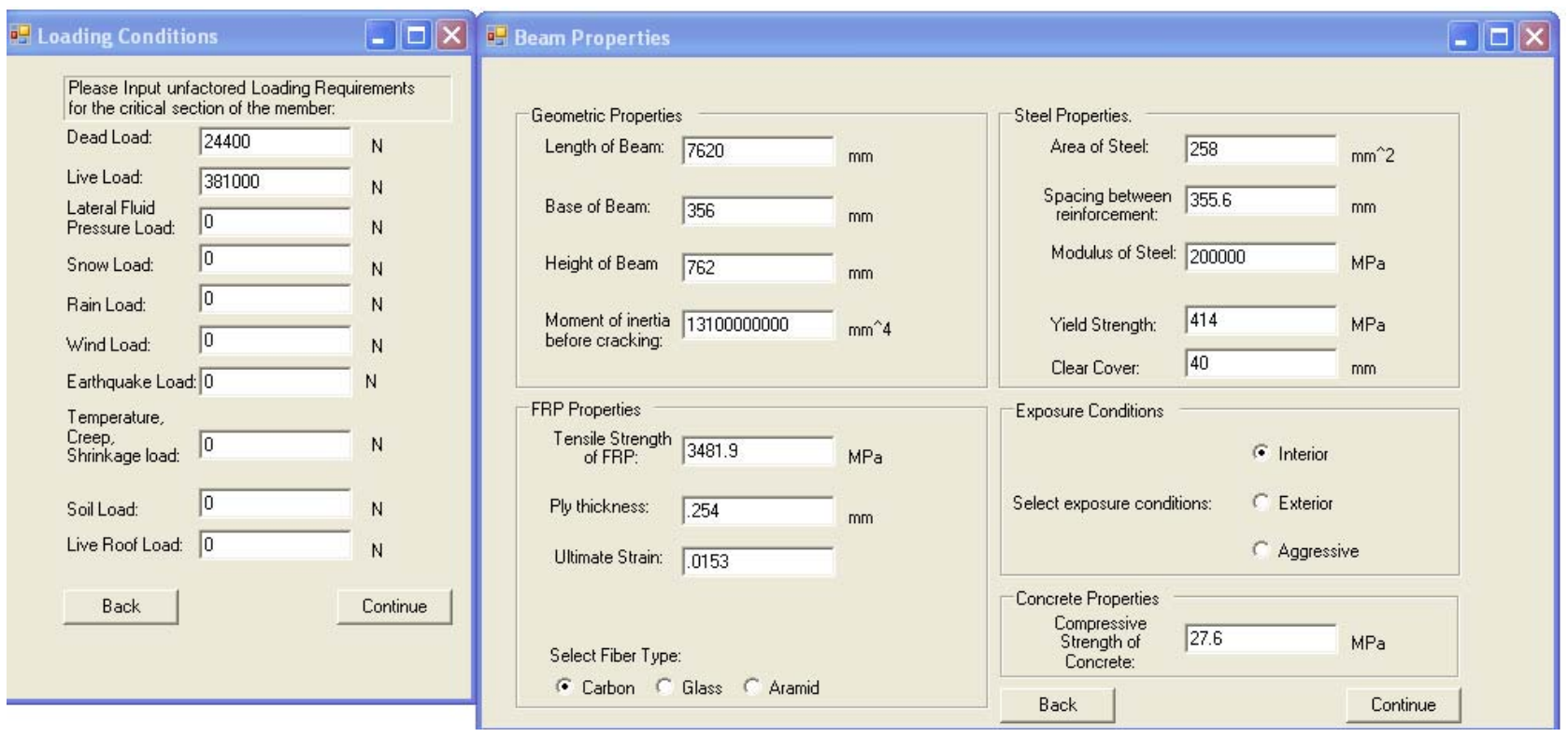

Figure 5.11 Example F: Windows Form Inputs 


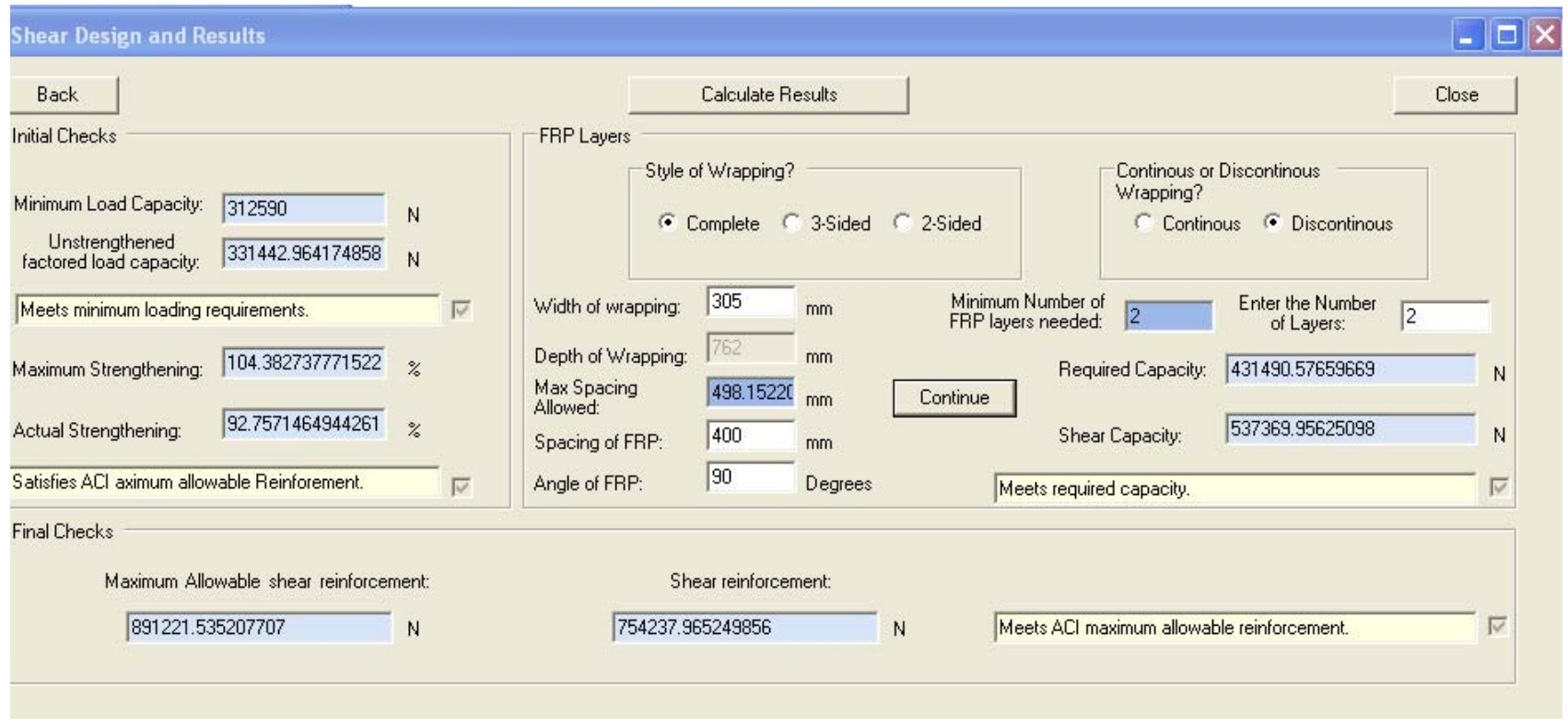

Figure 5.12 Example F Outputs

This example uses complete discontinuous wrapping scheme to strengthen. The width of the FRP was $305 \mathrm{~mm}$. The angle of application of the FRP was 90, and the spacing of the FRP strips was 400 $\mathrm{mm}$. The application and updated example both suggest using 2 layers. Table 5.12 shows the results.

\section{Table 5.12 Comparison of Example F Outputs}

\begin{tabular}{|l|c|c|c|}
\cline { 2 - 4 } \multicolumn{1}{c|}{} & $\begin{array}{c}\text { Updated } \\
\text { Example }\end{array}$ & Application & Difference \\
\hline Required capacity & $4.31 \mathrm{E}+05$ & $4.31 \mathrm{E}+05$ & $0.00 \mathrm{E}+00$ \\
\hline Factored capacity & $5.37 \mathrm{E}+05$ & $5.37 \mathrm{E}+05$ & $0.00 \mathrm{E}+00$ \\
\hline actual reinforcement & $7.54 \mathrm{E}+05$ & $7.54 \mathrm{E}+05$ & $0.00 \mathrm{E}+00$ \\
\hline
\end{tabular}

\subsubsection{Example G}

Example $\mathrm{G}$ is a rectangular beam with increased shear live loading [3]. Table 5.13 displays the parameters in terms of properties and loading. The parameters can be seen entered into the application in Figures 5.12 and 5.13. This example is updated with respect to [3] to meet current $\mathrm{ACl} 440.2 \mathrm{R}-08$ pratices. See Appendix A for hand calculations. 
Table 5.13 Example G Inputs

\begin{tabular}{|c|c|c|c|c|c|}
\hline \multicolumn{2}{|c|}{ Geometric Properties [3] } & \multicolumn{2}{|c|}{ Steel Properties [3] } & \multicolumn{2}{|l|}{ FRP Properties [3] } \\
\hline length [mm] & 4000 & Area of steel [mm^2] & 113.14 & FRP tensile strength [mPa] & 3481.9 \\
\hline base $[\mathrm{mm}]$ & 381 & Shear spacing $[\mathrm{mm}]$ & 200 & FRP ultimate strain & 0.0153 \\
\hline height [mm] & 635 & yield strength [mPa] & 275.79 & ply thickness [mm] & 0.254 \\
\hline Moment of Inertia $\mathrm{mm}^{\wedge} 4$ ] & $8.13 E+09$ & modulus of steel [mPa] & 200000 & Fiber & Carbon \\
\hline \multicolumn{2}{|c|}{ Loading Requirements [3] } & clear cover [mm] & 76.2 & \multicolumn{2}{|l|}{ Concrete Properties [3] } \\
\hline Dead Load [N-mm] & $9.20 \mathrm{E}+04$ & \multicolumn{2}{|l|}{ Exposure } & Concrete Strength [mPa] & 20.68 \\
\hline Live Load [N-mm] & $1.30 \mathrm{E}+05$ & \multicolumn{2}{|l|}{ interior } & & \\
\hline
\end{tabular}

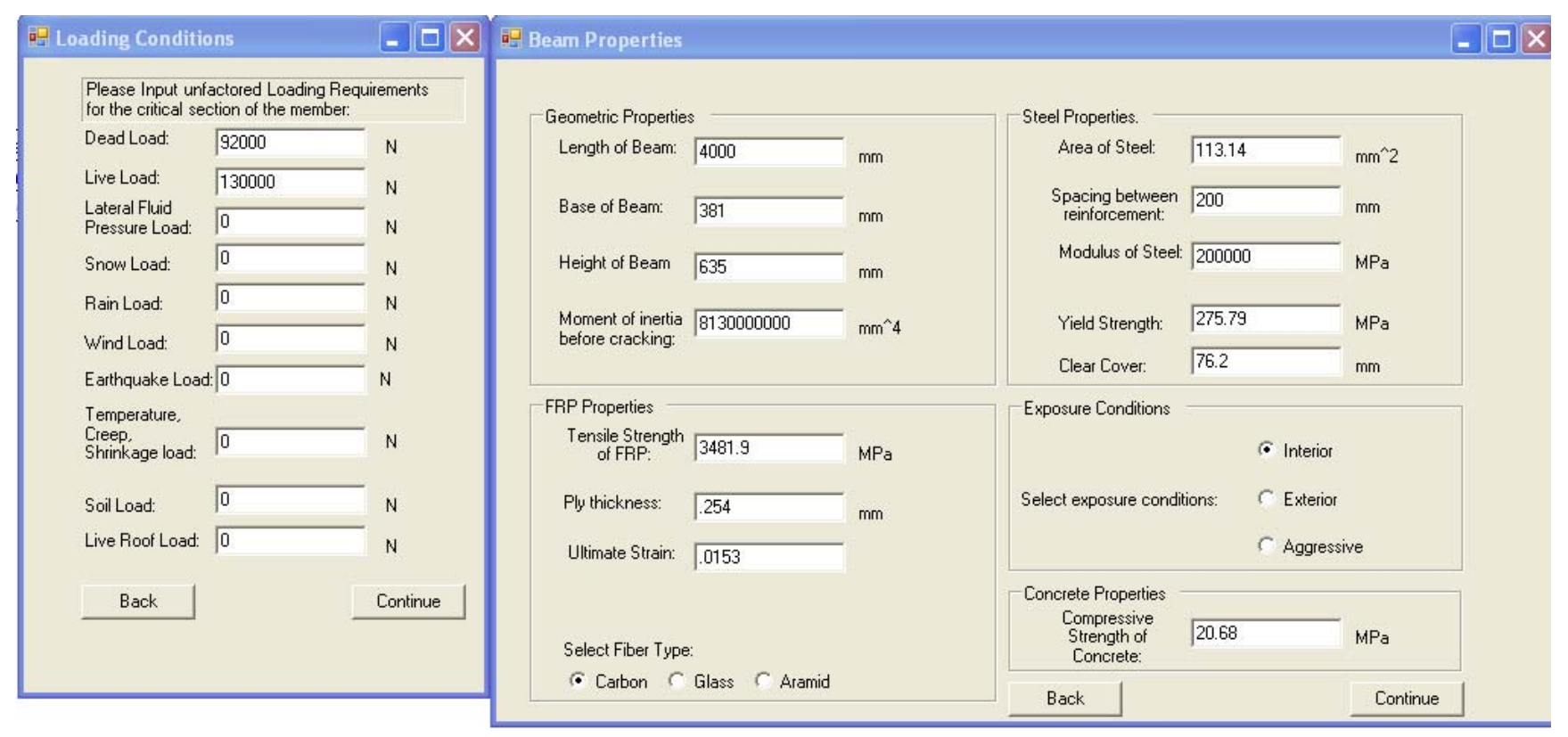

Figure 5.12 Example G: Windows Form Inputs 


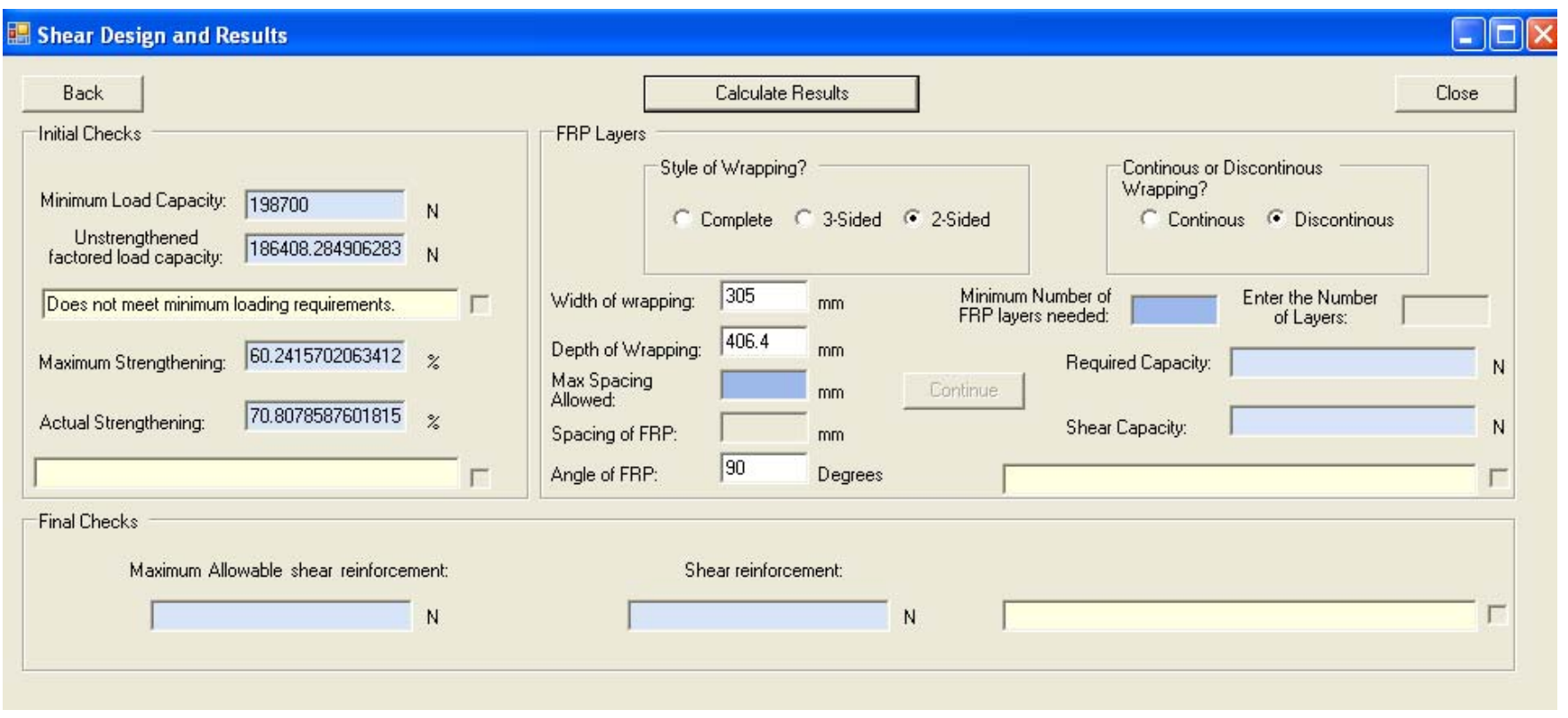

Figure 5.13 Example G Outputs

The application and the updated example agree that the minimum loading capacity was not met. Therefore, the application does not allow the user to continue the design and the strengthen capacity and the maximum allowable shear reinforcement are not calculated and can't be compared to the example which does calculate those values even though the beam does not satisfy the ACl's minimum load capacity. Table 5.13 shows the available outputs.

Table 5.13 Comparison of Example G Outputs

\begin{tabular}{|l|c|c|}
\cline { 2 - 3 } \multicolumn{1}{c|}{} & \multicolumn{1}{c|}{$\begin{array}{c}\text { Updated } \\
\text { Example }\end{array}$} & Application \\
\hline Required capacity & $2.07 \mathrm{E}+05$ & N/A \\
\hline Factored capacity & $1.42 \mathrm{E}+05$ & N/A \\
\hline actual reinforcement & $2.29 \mathrm{E}+05$ & N/A \\
\hline
\end{tabular}

\subsection{Strengthening Columns in Pure Axial Compression}

\subsubsection{Example $\mathrm{H}$}

Example $\mathrm{H}$ is a circular column experiencing a $50 \%$ increase in live loading in pure axial compression [4]. Table 5.14 displays the all required parameters of the problem. The parameters can 
also be seen in Figure 5.14 which shows the values as inputs in the application. Figure 5.15 displays the outputs of the application.

\section{Table 5.14 Example H Inputs}

\begin{tabular}{|c|c|c|c|c|c|}
\hline \multicolumn{2}{|c|}{ Geometric Properties [4] } & \multicolumn{2}{|c|}{ Steel Properties [4] } & \multicolumn{2}{|l|}{ FRP Properties [4] } \\
\hline length [mm] & 5000 & Area of steel [mm^2] & 1061 & FRP tensile strength [mPa] & 3300 \\
\hline diameter [mm] & 450 & yield strength [mPa] & 275 & FRP ultimate strain & 0.016 \\
\hline base $[\mathrm{mm}]$ & 0 & modulus of steel [mPa] & 200000 & ply thickness [mm] & 0.25 \\
\hline height [mm] & 0 & Reinforcement style & Sprial & Fiber & Carbon \\
\hline \multicolumn{2}{|c|}{ Loading Requirements [4] } & \multicolumn{2}{|l|}{ Exposure } & \multicolumn{2}{|l|}{ Concrete Properties [4] } \\
\hline Dead Load [N-mm] & $9.50 \mathrm{E}+05$ & \multicolumn{2}{|l|}{ interior } & Concrete Strength [mPa] & 27.57 \\
\hline Live Load [N-mm] & $1.28 \mathrm{E}+06$ & & & & \\
\hline
\end{tabular}

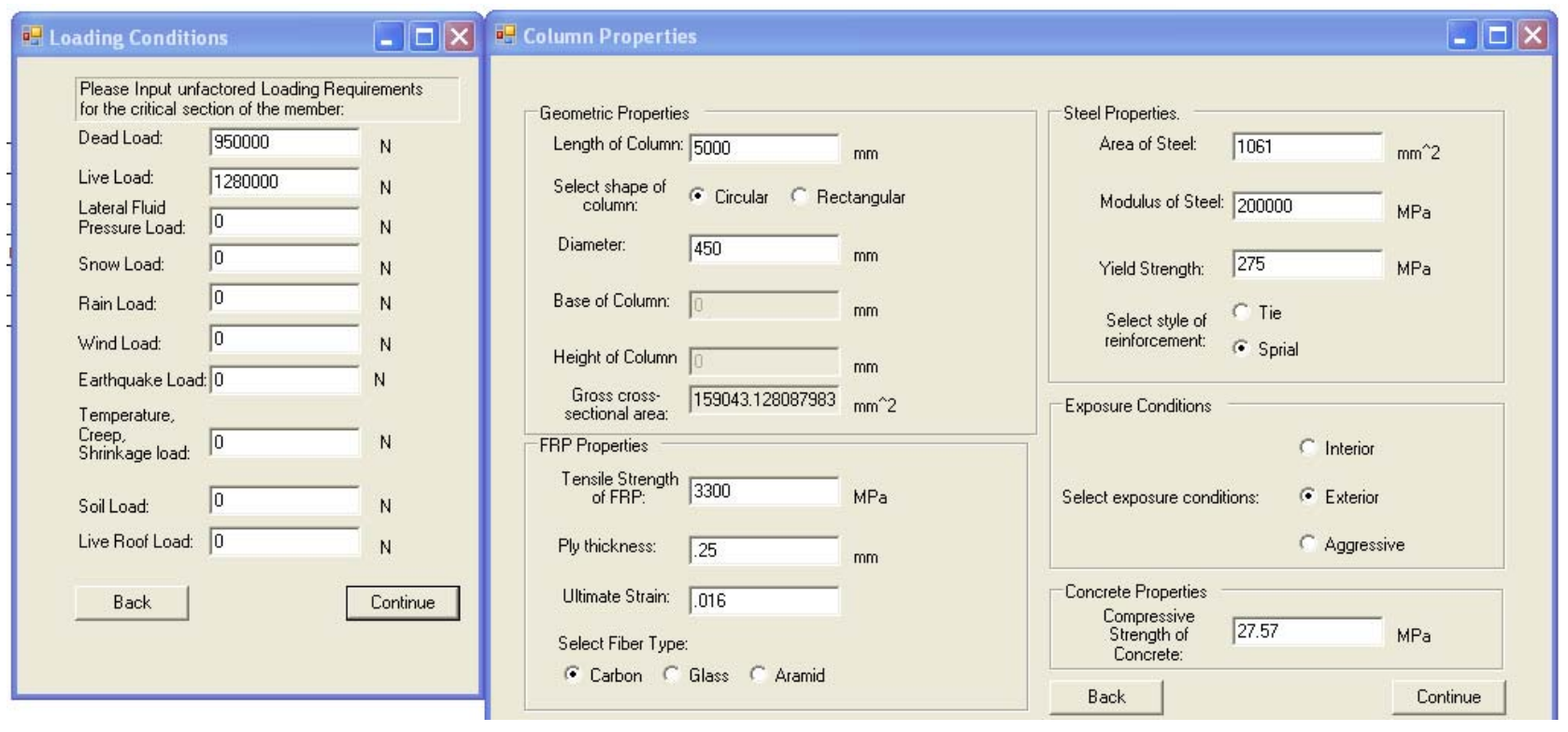

Figure 5.14 Example H: Windows Form Inputs 


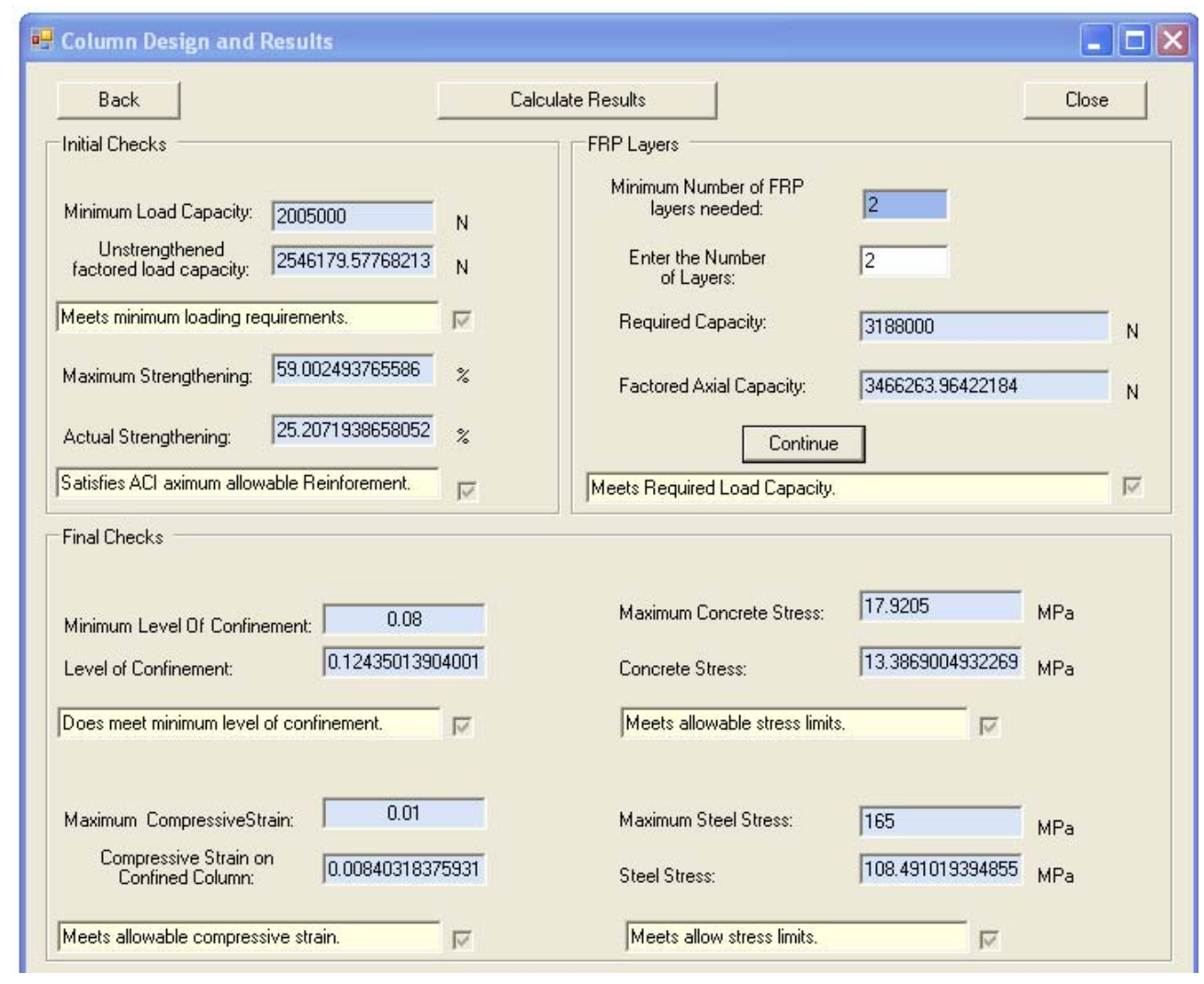

Figure 5.15 Example H Outputs

The example and the application suggest using 2 layers of FRP to meet the required loading capacity. The minimum confinement ratio, maximum compression strain, and concrete and steel stress levels all meet requirements in the application and the updated example. Table 5.15 shows a comparison of the calculated results.

\section{Table 5.15 Comparison of Example H Outputs}

\begin{tabular}{|l|c|c|c|}
\cline { 2 - 4 } \multicolumn{1}{c|}{} & \multicolumn{1}{c|}{$\begin{array}{c}\text { Updated } \\
\text { Example }\end{array}$} & Application & Difference \\
\hline Required capacity & $3.18 \mathrm{E}+06$ & $3.19 \mathrm{E}+06$ & $-1.00 \mathrm{E}+04$ \\
\hline Factored capacity & $3.47 \mathrm{E}+06$ & $3.47 \mathrm{E}+06$ & $0.00 \mathrm{E}+00$ \\
\hline Confinement Ratio & 0.124 & 0.124 & $0.00 \mathrm{E}+00$ \\
\hline $\begin{array}{l}\text { Confined concrete } \\
\text { strain }\end{array}$ & 0.0084 & 0.0084 & $0.00 \mathrm{E}+00$ \\
\hline Concrete Stress & 13.357 & 13.386 & $-2.90 \mathrm{E}-02$ \\
\hline Steel Stress & 108.248 & 108.491 & $-2.43 \mathrm{E}-01$ \\
\hline
\end{tabular}




\subsubsection{Example I}

Example I is also a circular concrete column that needs strengthened to support the increase of loading [3]. All the required properties and loading combinations are located in Table 5.16. Figures 5.16 and 5.17 display the properties and loading combinations entered in the application and the outputs of the application. This example is updated with respect to [3] to meet current ACl 440.2R-08 pratices. See Appendix A for hand calculations.

\section{Table 5.16 Example I Inputs}

\begin{tabular}{|c|c|c|c|c|c|}
\hline \multicolumn{2}{|c|}{ Geometric Properties [3] } & \multicolumn{2}{|c|}{ Steel Properties [3] } & \multicolumn{2}{|l|}{ FRP Properties [3] } \\
\hline length [mm] & 4000 & Area of steel [mm^2] & 7045 & FRP tensile strength [mPa] & 3792 \\
\hline diameter $[\mathrm{mm}]$ & 508 & yield strength [mPa] & 414 & FRP ultimate strain & 0.0167 \\
\hline base $[\mathrm{mm}]$ & 0 & modulus of steel [mPa] & 200000 & ply thickness [mm] & 0.1651 \\
\hline height [mm] & 0 & Reinforcement style & Sprial & Fiber & Carbon \\
\hline \multicolumn{2}{|c|}{ Loading Requirements [3] } & \multicolumn{2}{|l|}{ Exposure } & \multicolumn{2}{|l|}{ Concrete Properties [3] } \\
\hline Dead Load [N-mm] & $1.42 \mathrm{E}+06$ & \multicolumn{2}{|l|}{ interior } & Concrete Strength [mPa] & 27.6 \\
\hline Live Load [N-mm] & $2.56 \mathrm{E}+06$ & & & & \\
\hline
\end{tabular}

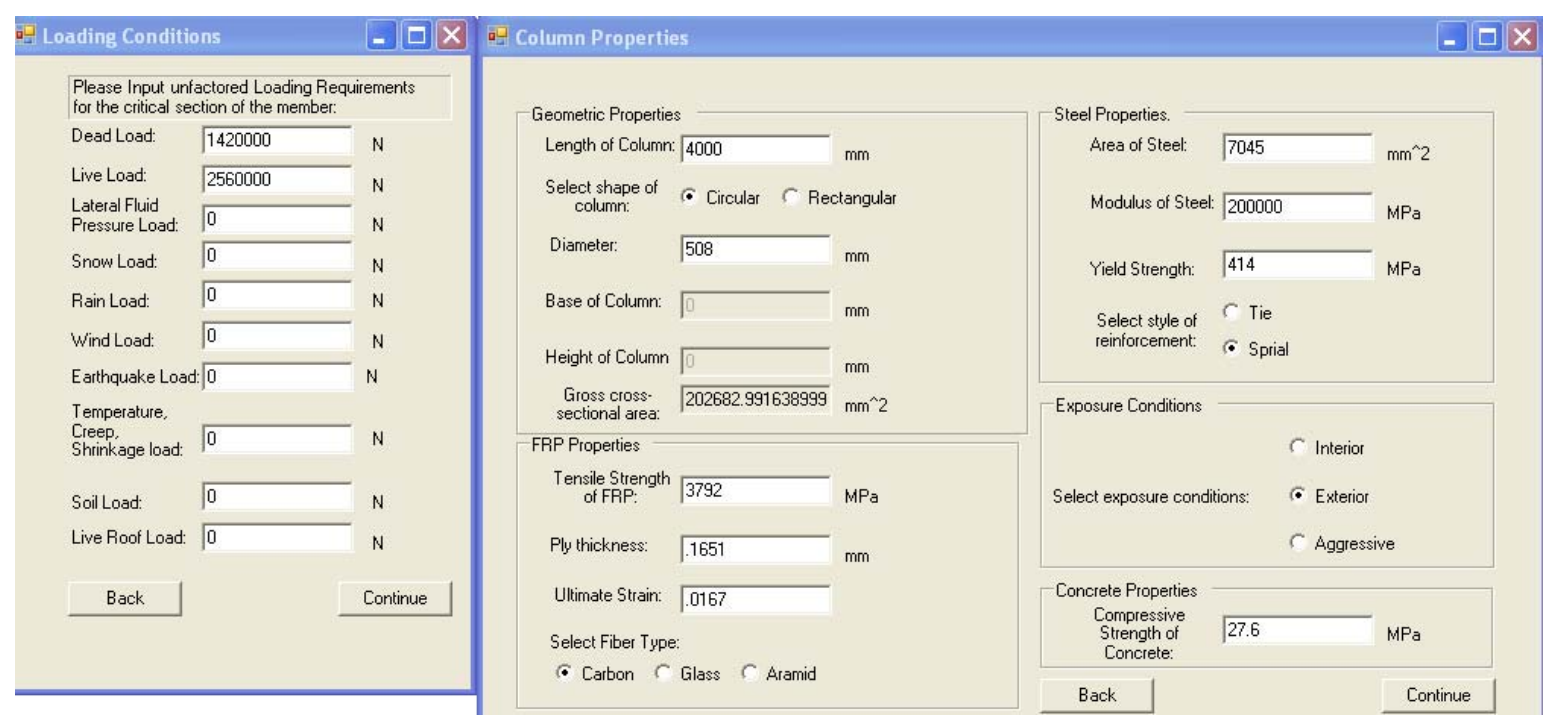

Figure 5.16 Example I: Windows Form Inputs 


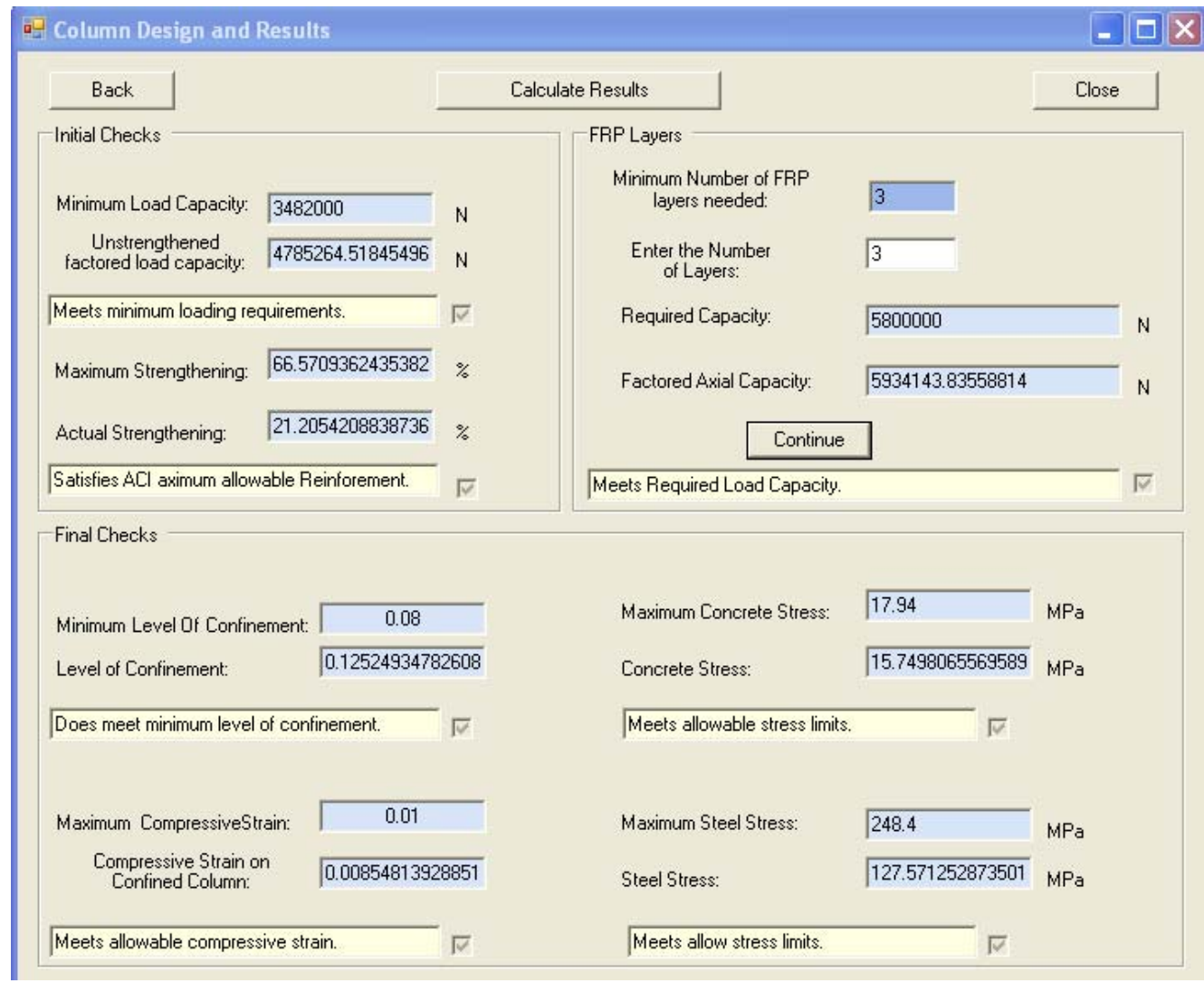

Figure 5.17 Example I Outputs

Three layers of FRP were suggested to meet the required capacity by both the updated example and the application. Also, all the requirements for capacity and $\mathrm{ACl}$ serviceability were met by both. A comparison of these values can be seen in Table 5.17.

Table 5.17 Comparison of Example I Outputs

\begin{tabular}{|l|c|c|c|}
\cline { 2 - 4 } \multicolumn{1}{c|}{} & \multicolumn{1}{c|}{$\begin{array}{c}\text { Updated } \\
\text { Example }\end{array}$} & Application & Difference \\
\hline Required capacity & $5.81 \mathrm{E}+06$ & $5.80 \mathrm{E}+06$ & $1.00 \mathrm{E}+04$ \\
\hline Factored capacity & $5.93 \mathrm{E}+06$ & $5.93 \mathrm{E}+06$ & $0.00 \mathrm{E}+00$ \\
\hline Confinement Ratio & 0.125 & 0.125 & $0.00 \mathrm{E}+00$ \\
\hline $\begin{array}{l}\text { Confined concrete } \\
\text { strain }\end{array}$ & 0.009 & 0.00854 & $4.60 \mathrm{E}-04$ \\
\hline Concrete Stress & 15.771 & 15.75 & $2.10 \mathrm{E}-02$ \\
\hline Steel Stress & 127.745 & 127.571 & $1.74 \mathrm{E}-01$ \\
\hline
\end{tabular}




\subsubsection{Example J}

Example $\mathrm{J}$ is a rectangular concrete column with an increase loading causing a need for strengthening using FRP [6]. The properties of the column and the loads are in Table 5.18. The values are also entered in the application in Figure 5.18. Figure 5.19 displays the outputs of the application. This example is updated with respect to [6] to meet current $\mathrm{ACl} 440.2 \mathrm{R}-08$ pratices and increased required loading. See Appendix A for hand calculations.

\section{Table 5.18 Example J Inputs}

\begin{tabular}{|c|c|c|c|c|c|}
\hline \multicolumn{2}{|c|}{ Geometric Properties [6] } & \multicolumn{2}{|c|}{ Steel Properties [6] } & \multicolumn{2}{|l|}{ FRP Properties } \\
\hline length [mm] & 5000 & Area of steel $\left[\mathrm{mm}^{\wedge} 2\right]$ & 6774.6 & FRP tensile strength [mPa] & 3300 \\
\hline diameter $[\mathrm{mm}]$ & 0 & yield strength [mPa] & 414 & FRP ultimate strain & 0.016 \\
\hline base $[\mathrm{mm}]$ & 508 & modulus of steel [mPa] & 200000 & ply thickness [mm] & 0.25 \\
\hline height [mm] & 571.5 & Reinforcement style & Tie & Fiber & Carbon \\
\hline \multicolumn{2}{|c|}{ Loading Requirements } & \multicolumn{2}{|l|}{ Exposure } & \multicolumn{2}{|l|}{ Concrete Properties [6] } \\
\hline Dead Load [N-mm] & $5.67 \mathrm{E}+05$ & \multicolumn{2}{|l|}{ interior } & Concrete Strength [mPa] & 27.6 \\
\hline Live Load [N-mm] & $2.78 \mathrm{E}+06$ & & & & \\
\hline
\end{tabular}

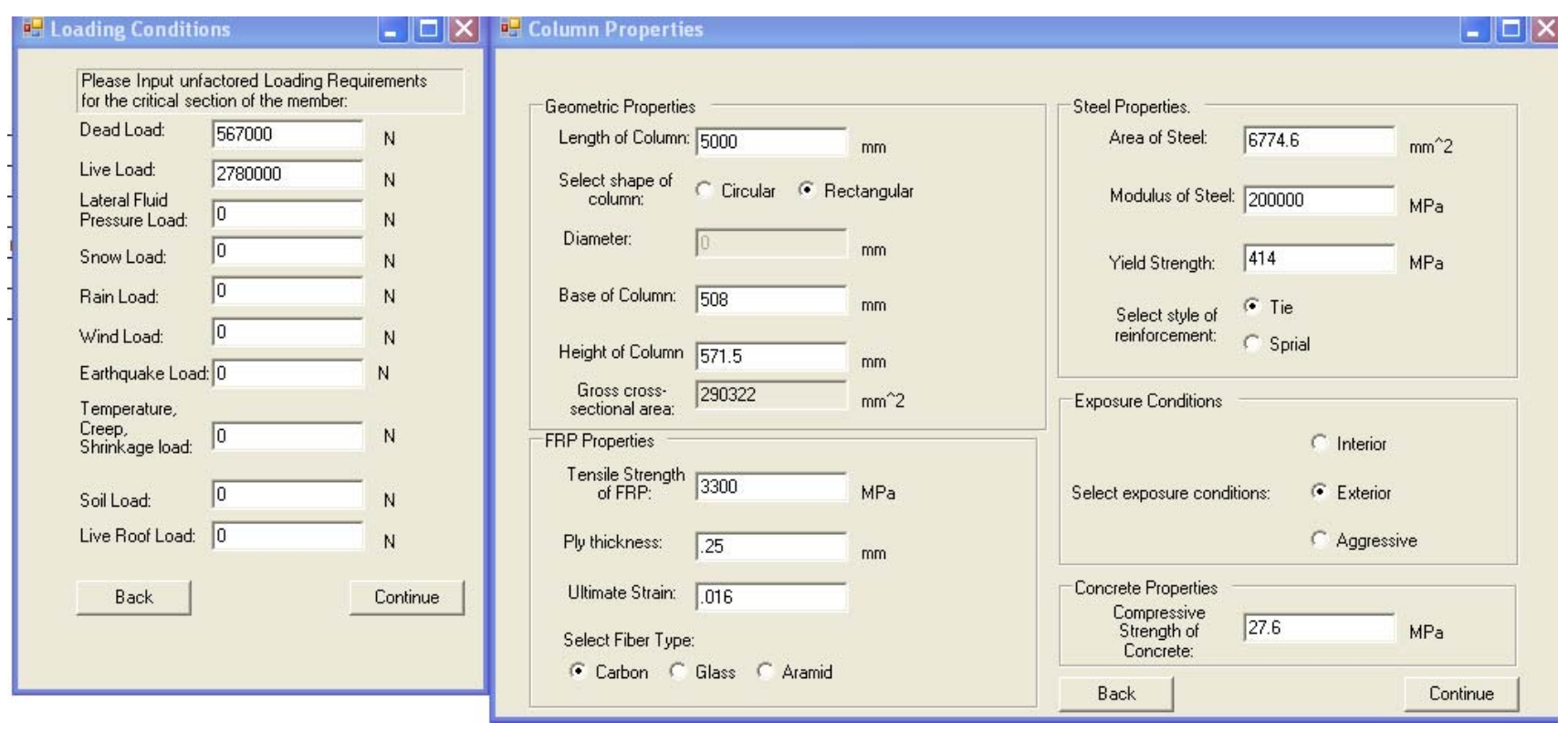

Figure 5.18 Example: J Windows Form Inputs 


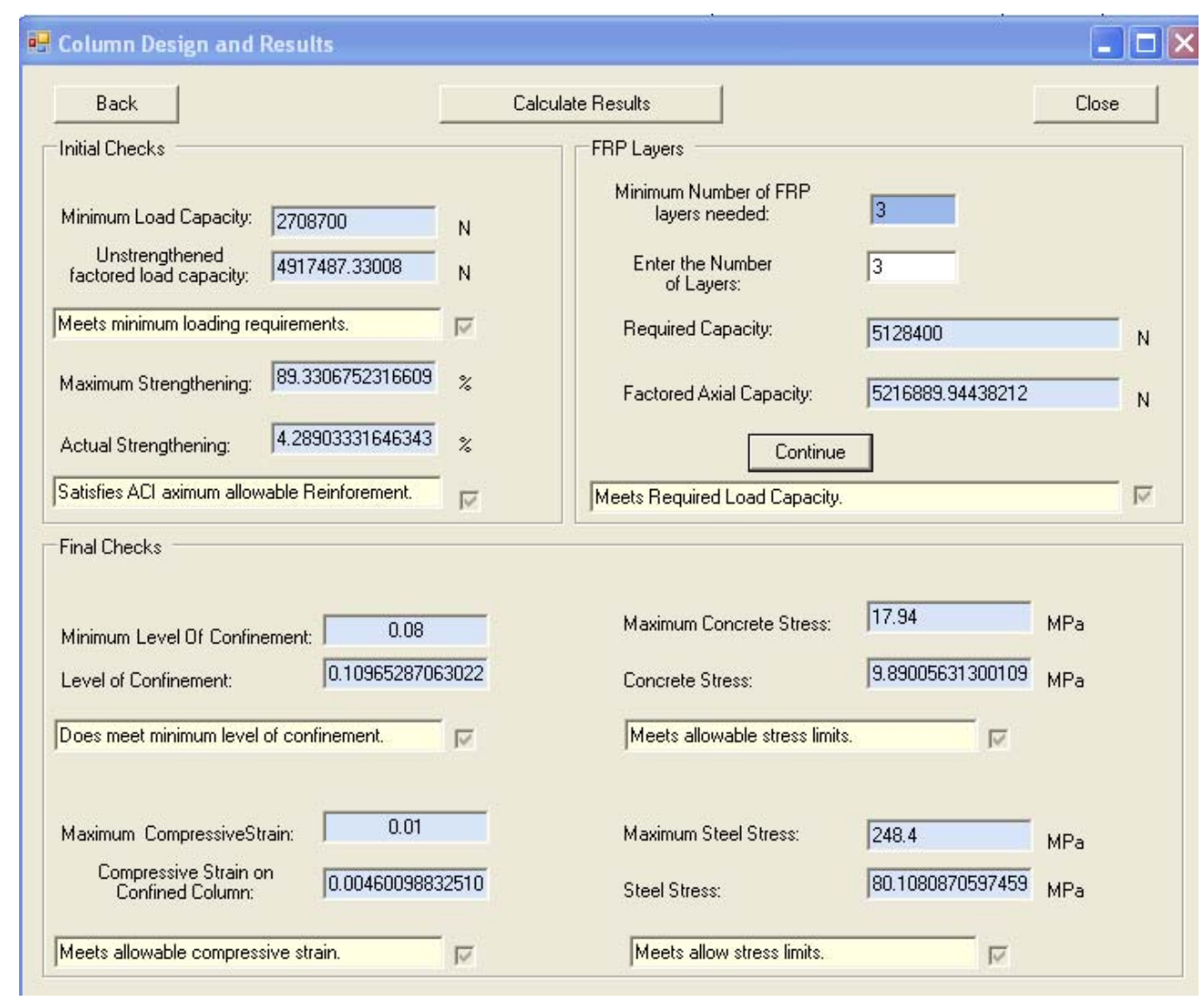

Figure 5.19 Example J Outputs

The example and the application suggest using 3 layers to meet the required capacity. Using 3

layers, all required capacities and serviceability limits were met by the updated example and the application outputs. These values can be seen in Table 5.19.

\section{Table 5.19 Comparison of Example I Outputs}

\begin{tabular}{|l|c|c|c|}
\cline { 2 - 4 } \multicolumn{1}{c|}{} & $\begin{array}{c}\text { Updated } \\
\text { Example }\end{array}$ & Application & Difference \\
\hline Required capacity & $5.13 \mathrm{E}+06$ & $5.13 \mathrm{E}+06$ & $0.00 \mathrm{E}+00$ \\
\hline Factored capacity & $5.22 \mathrm{E}+06$ & $5.22 \mathrm{E}+06$ & $0.00 \mathrm{E}+00$ \\
\hline Confinement Ratio & 0.11 & 0.1096 & $4.00 \mathrm{E}-04$ \\
\hline Confined concrete strain & 0.0046 & 0.0046 & $0.00 \mathrm{E}+00$ \\
\hline Concrete Stress & 9.89 & 9.89 & $0.00 \mathrm{E}+00$ \\
\hline Steel Stress & 80.108 & 80.108 & $0.00 \mathrm{E}+00$ \\
\hline
\end{tabular}




\subsection{Strengthening Beam-Columns}

\subsubsection{Example $\mathrm{K}$}

Example $\mathrm{K}$ is a concrete column experiencing axial and moment loading. Tthe given

strengthening design must be verified by creating an interaction diagram for the column [4]. Table 5.20

shows all the parameters of the example. Figure 5.20 displays these values in the application while

Figure 5.21 is the interaction diagram produced by the application.

\section{Table 5.20 Example K Inputs}

\begin{tabular}{|c|c|c|c|c|c|}
\hline \multicolumn{2}{|c|}{ Geometric Properties [4] } & \multicolumn{2}{|c|}{ Steel Properties [4] } & \multicolumn{2}{|c|}{ FRP Properties [4] } \\
\hline length [mm] & 5000 & Distance of Steel [mm] & $40,185,315,460$ & $\begin{array}{l}\text { FRP tensile strength } \\
\qquad[\mathrm{mPa}]\end{array}$ & 3792 \\
\hline diameter $[\mathrm{mm}]$ & 0 & Area of steel [mm^2] & $\begin{array}{c}1520,760,760 \\
1520\end{array}$ & FRP ultimate strain & 0.0166 \\
\hline base $[\mathrm{mm}]$ & 500 & yield strength [mPa] & 414 & ply thickness [mm] & 0.333 \\
\hline height [mm] & 500 & modulus of steel [mPa] & 200000 & number of layers & 3 \\
\hline \multicolumn{2}{|c|}{ Loading Requirements [4] } & Reinforcement style & Tie & Fiber & Carbon \\
\hline Factored Axial Load [N] & $5.20 \mathrm{E}+06$ & clear cover [mm] & 40 & \multicolumn{2}{|c|}{ Concrete Properties [4] } \\
\hline $\begin{array}{l}\text { Factored Moment [N- } \\
\mathrm{mm}]\end{array}$ & $4.50 \mathrm{E}+08$ & \multicolumn{2}{|c|}{ Exposure } & $\begin{array}{c}\text { Concrete Strength } \\
{[\mathrm{mPa}]}\end{array}$ & 27.6 \\
\hline
\end{tabular}




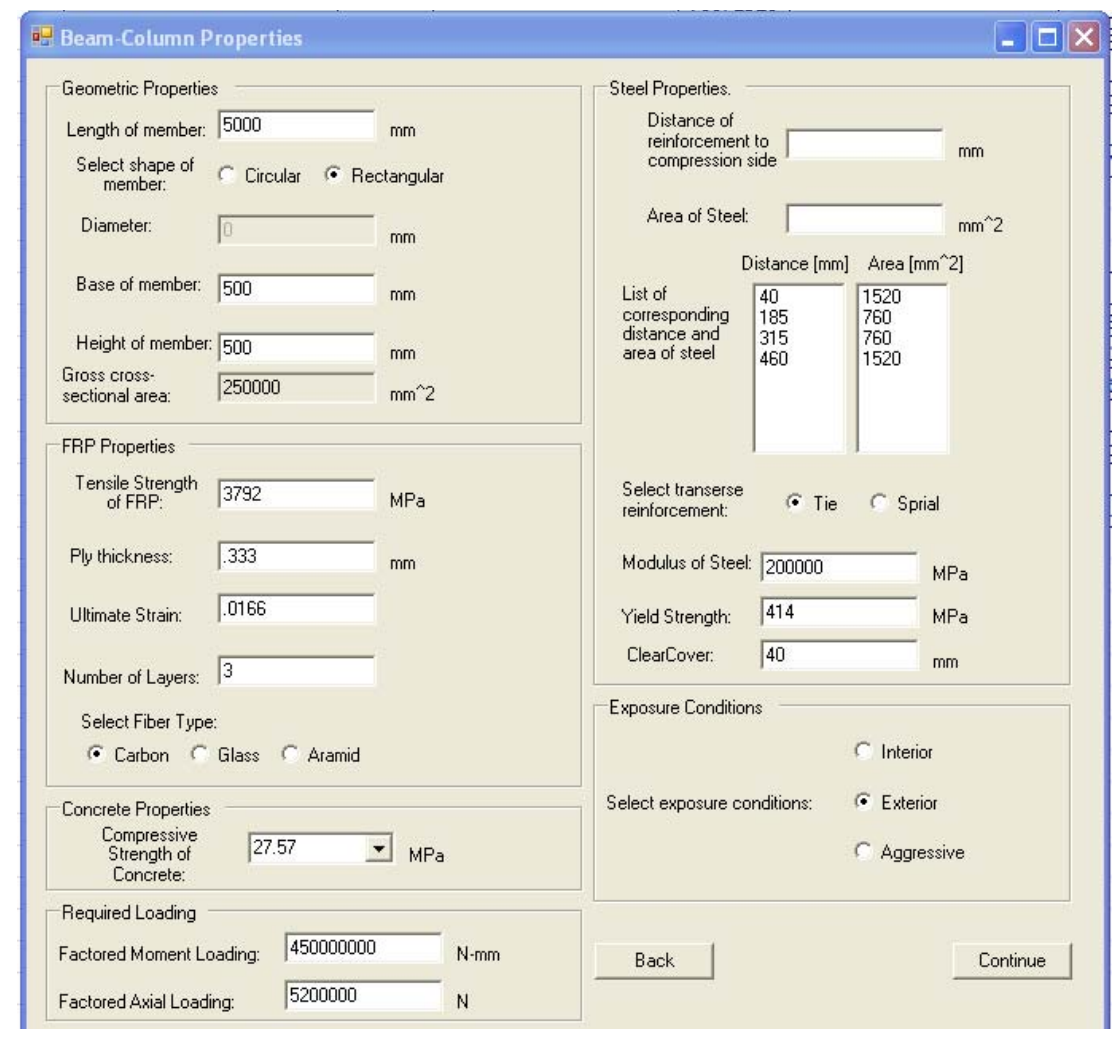

Figure 5.20 Example K: Windows Form Inputs

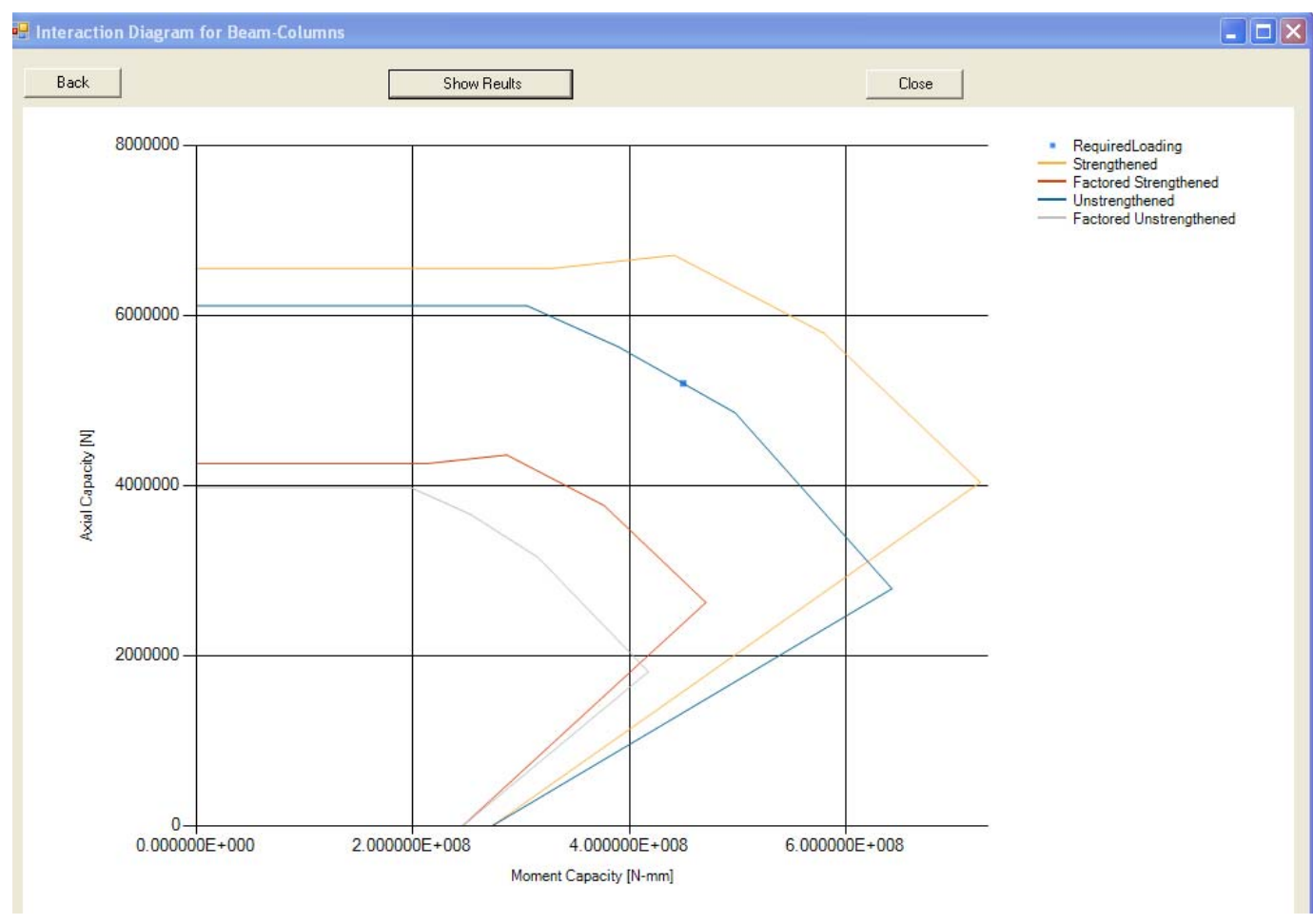

Figure 5.21 Example K: Interaction Diagram 
The verification process for Example $\mathrm{K}$ produces six critical points on the interaction diagram for four different lines: nominal and factored axial capacity, strengthen nominal and factored axial capacity, nominal and factored moment capacity, and strengthen nominal and factored moment capacity. Table 5.21 compares the values of these six points from the updated example to the application outputs. This example is updated with respect to [4] to meet current ACl 440.2R-08 pratices. See Appendix A for hand calculations.

\section{Table 5.21 Comparison of Example K Outputs}

\begin{tabular}{|c|l|c|c|l|l|l|l|}
\cline { 2 - 8 } \multicolumn{2}{c|}{} & Point A & Point D & Point B & Point C & Point E & Point F \\
\hline \multirow{3}{*}{ Pn } & Example & $6.12 \mathrm{E}+06$ & $6.12 \mathrm{E}+06$ & $5.63 \mathrm{E}+06$ & $2.79 \mathrm{E}+06$ & $4.85 \mathrm{E}+06$ & $0.00 \mathrm{E}+00$ \\
\cline { 2 - 8 } & Application & $6.11 \mathrm{E}+06$ & $6.12 \mathrm{E}+05$ & $5.63 \mathrm{E}+06$ & $2.79 \mathrm{E}+06$ & $4.85 \mathrm{E}+06$ & $0.00 \mathrm{E}+00$ \\
\hline \multirow{3}{*}{$\Phi$ Pn } & Example & $3.97 \mathrm{E}+06$ & $3.97 \mathrm{E}+06$ & $3.66 \mathrm{E}+06$ & $1.81 \mathrm{E}+06$ & $3.16 \mathrm{E}+06$ & $0.00 \mathrm{E}+00$ \\
\cline { 2 - 9 } & Application & $3.97 \mathrm{E}+06$ & $3.97 \mathrm{E}+06$ & $3.66 \mathrm{E}+06$ & $1.81 \mathrm{E}+06$ & $3.15 \mathrm{E}+06$ & $0.00 \mathrm{E}+00$ \\
\hline \multirow{3}{*}{ Pstrn } & Example & $6.55 \mathrm{E}+06$ & $6.55 \mathrm{E}+06$ & $6.69 \mathrm{E}+06$ & $4.01 \mathrm{E}+06$ & $5.69 \mathrm{E}+06$ & $0.00 \mathrm{E}+00$ \\
\cline { 2 - 9 } & Application & $6.55 \mathrm{E}+06$ & $6.55 \mathrm{E}+06$ & $6.70 \mathrm{E}+06$ & $4.04 \mathrm{E}+06$ & $5.79 \mathrm{E}+06$ & $0.00 \mathrm{E}+00$ \\
\hline \multirow{3}{*}{ Pstrn } & Example & $4.26 \mathrm{E}+06$ & $4.26 \mathrm{E}+06$ & $4.35 \mathrm{E}+06$ & $2.60 \mathrm{E}+06$ & $3.70 \mathrm{E}+06$ & $0.00 \mathrm{E}+00$ \\
\cline { 2 - 8 } & Application & $4.26 \mathrm{E}+06$ & $4.26 \mathrm{E}+06$ & $4.36 \mathrm{E}+06$ & $2.62 \mathrm{E}+06$ & $3.76 \mathrm{E}+06$ & $0.00 \mathrm{E}+00$ \\
\hline \multirow{3}{*}{ Mn } & Example & $0.00 \mathrm{E}+00$ & $3.06 \mathrm{E}+08$ & $3.90 \mathrm{E}+08$ & $6.43 \mathrm{E}+08$ & $4.86 \mathrm{E}+08$ & $2.79 \mathrm{E}+08$ \\
\cline { 2 - 9 } & Application & $0.00 \mathrm{E}+00$ & $3.06 \mathrm{E}+08$ & $3.90 \mathrm{E}+07$ & $6.43 \mathrm{E}+08$ & $4.98 \mathrm{E}+08$ & $2.74 \mathrm{E}+08$ \\
\hline \multirow{3}{*}{ MMn } & Example & $0.00 \mathrm{E}+00$ & $1.99 \mathrm{E}+08$ & $2.54 \mathrm{E}+08$ & $4.18 \mathrm{E}+08$ & $3.16 \mathrm{E}+08$ & $2.51 \mathrm{E}+08$ \\
\cline { 2 - 9 } & Application & $0.00 \mathrm{E}+00$ & $1.99 \mathrm{E}+08$ & $2.54 \mathrm{E}+08$ & $4.18 \mathrm{E}+07$ & $3.16 \mathrm{E}+08$ & $2.46 \mathrm{E}+08$ \\
\hline \multirow{3}{*}{ Mstrn } & Example & $0.00 \mathrm{E}+00$ & $3.27 \mathrm{E}+08$ & $4.41 \mathrm{E}+08$ & $7.23 \mathrm{E}+08$ & $5.72 \mathrm{E}+08$ & $2.79 \mathrm{E}+08$ \\
\cline { 2 - 8 } & Application & $0.00 \mathrm{E}+00$ & $3.27 \mathrm{E}+08$ & $4.41 \mathrm{E}+08$ & $7.24 \mathrm{E}+08$ & $5.80 \mathrm{E}+08$ & $2.74 \mathrm{E}+08$ \\
\hline \multirow{2}{*}{ Mstrn } & Example & $0.00 \mathrm{E}+00$ & $2.13 \mathrm{E}+08$ & $2.87 \mathrm{E}+08$ & $4.70 \mathrm{E}+08$ & $3.72 \mathrm{E}+08$ & $2.51 \mathrm{E}+08$ \\
\cline { 2 - 8 } & Application & $0.00 \mathrm{E}+00$ & $2.13 \mathrm{E}+08$ & $2.87 \mathrm{E}+08$ & $4.71 \mathrm{E}+08$ & $3.77 \mathrm{E}+08$ & $2.46 \mathrm{E}+08$ \\
\hline
\end{tabular}

\subsubsection{Example $L$}

Example $L$ is a rectangular column undergoing axial compression and bending [3]. The strengthening design must be verified using an interaction diagram. The example properties and other parameters are located in Table 5.22. Figure 5.22 also shows these values entered into the application. The interaction diagram produced by the application can be seen in Figure 5.23. The six critical points of 
the interaction diagram for the updated example and the application calculated is compared in Table

5.23. This example is updated with respect to [3] to meet current $\mathrm{ACl} 440.2 \mathrm{R}-08$ pratices. See Appendix

A for hand calculations.

\section{Table 5.22 Example L Inputs}

\begin{tabular}{|c|c|c|c|c|c|}
\hline \multicolumn{2}{|c|}{ Geometric Properties [3] } & \multicolumn{2}{|c|}{ Steel Properties [3] } & \multirow{2}{*}{$\begin{array}{c}\text { FRP Properties [3] } \\
\text { FRP tensile strength } \\
\text { [mPa] }\end{array}$} & \multirow[b]{2}{*}{3792} \\
\hline length [mm] & 5000 & Distance of Steel [mm] & $76.2,279.4$ & & \\
\hline diameter $[\mathrm{mm}]$ & 0 & Area of steel $\left[\mathrm{mm}^{\wedge} 2\right]$ & 2013, 2013 & FRP ultimate strain & 0.017 \\
\hline base $[\mathrm{mm}]$ & 305 & yield strength [mPa] & 414 & ply thickness [mm] & 0.1 \\
\hline height $[\mathrm{mm}]$ & 355.6 & modulus of steel [mPa] & 200000 & number of layers & 3 \\
\hline \multicolumn{2}{|c|}{ Loading Requirements [3] } & Reinforcement style & Tie & Fiber & Carbon \\
\hline Factored Axial Load [N] & $5.20 \mathrm{E}+05$ & clear cover [mm] & 76.2 & \multicolumn{2}{|c|}{ Concrete Properties [3] } \\
\hline $\begin{array}{l}\text { Factored Moment [N- } \\
\mathrm{mm}]\end{array}$ & $4.50 E+07$ & \multicolumn{2}{|c|}{ Exposure } & Concrete Strength [mPa] & 34.5 \\
\hline
\end{tabular}

\begin{tabular}{l|l} 
interior \\
\hline
\end{tabular}

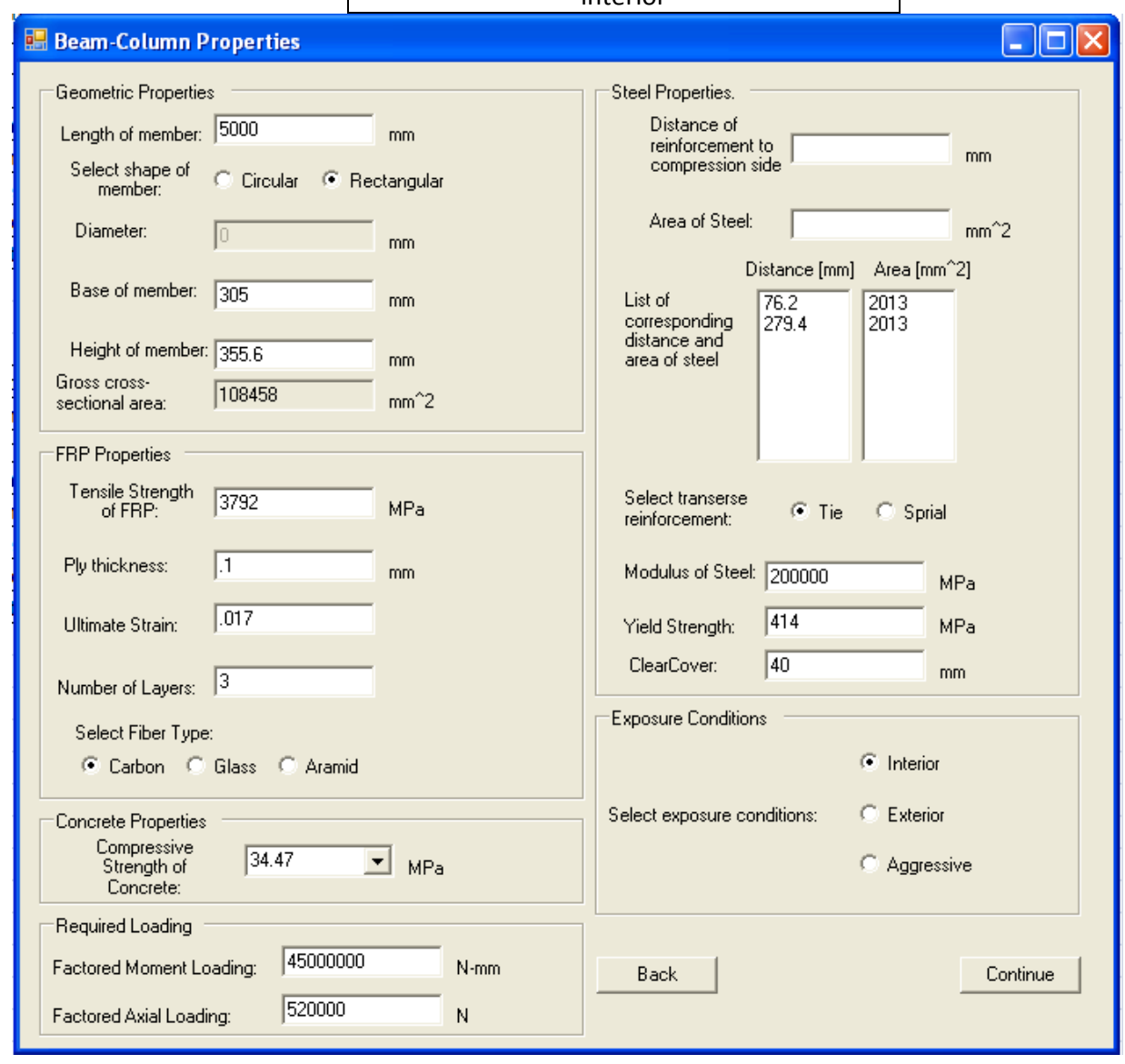

Figure 5.22 Example L: Windows Form Inputs 


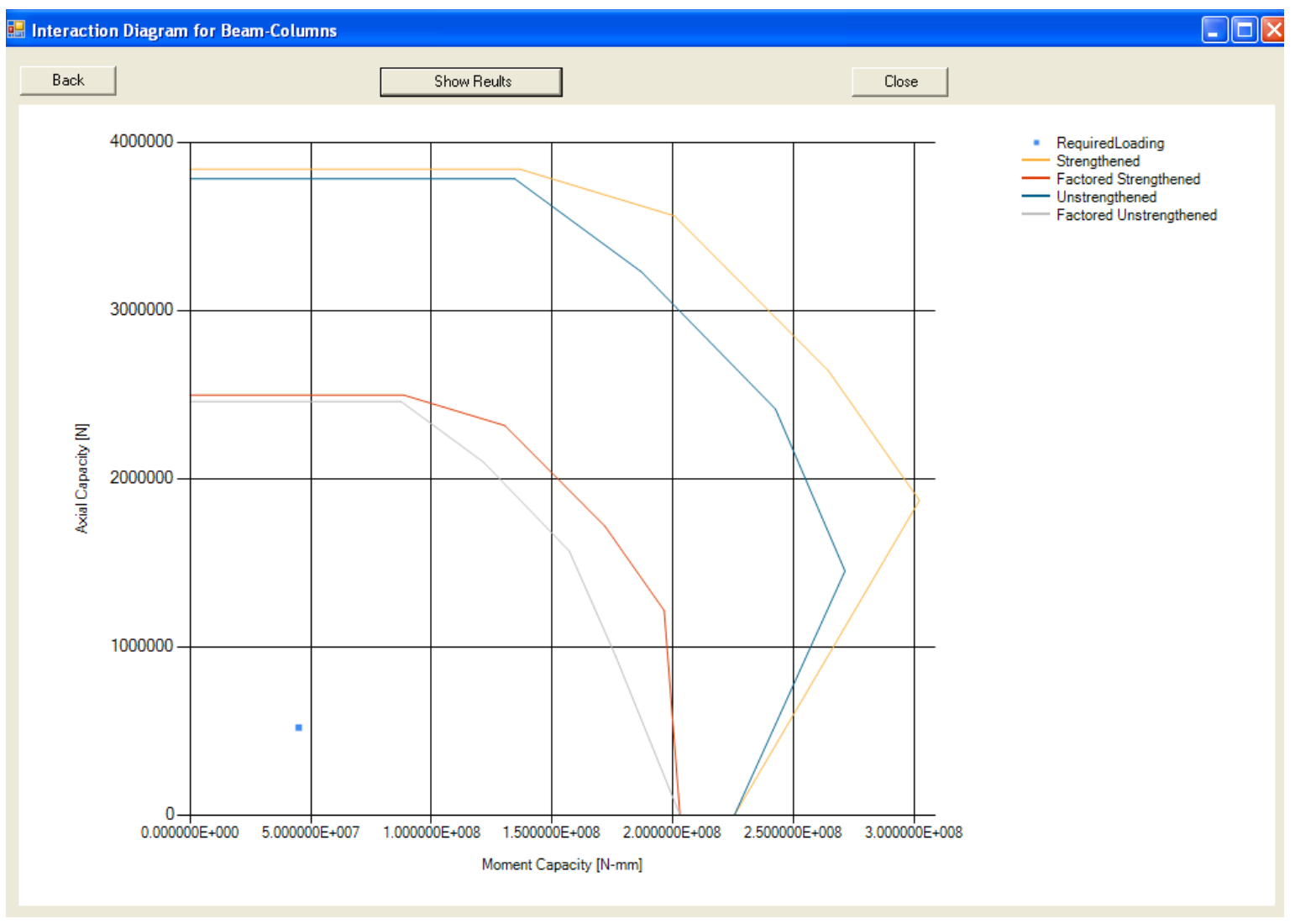

Figure 5.23 Example L: Interaction Diagram

Table 5.23 Comparison of Example L Outputs

\begin{tabular}{|c|l|c|c|c|c|c|c|}
\cline { 3 - 8 } \multicolumn{2}{c|}{} & Point A & Point D & Point B & Point C & Point E & Point F \\
\hline \multirow{2}{*}{ Pn } & Example & $3.78 \mathrm{E}+06$ & $3.78 \mathrm{E}+06$ & $2.83 \mathrm{E}+06$ & $1.00 \mathrm{E}+06$ & $2.41 \mathrm{E}+06$ & $0.00 \mathrm{E}+00$ \\
\cline { 2 - 8 } & Application & $3.78 \mathrm{E}+06$ & $3.78 \mathrm{E}+06$ & $2.83 \mathrm{E}+06$ & $1.00 \mathrm{E}+06$ & $2.41 \mathrm{E}+06$ & $0.00 \mathrm{E}+00$ \\
\hline \multirow{2}{*}{ DPn } & Example & $2.46 \mathrm{E}+06$ & $2.46 \mathrm{E}+06$ & $1.84 \mathrm{E}+06$ & $6.50 \mathrm{E}+05$ & $1.57 \mathrm{E}+06$ & $0.00 \mathrm{E}+00$ \\
\cline { 2 - 8 } & Application & $2.46 \mathrm{E}+06$ & $2.46 \mathrm{E}+06$ & $1.84 \mathrm{E}+06$ & $6.50 \mathrm{E}+05$ & $1.57 \mathrm{E}+05$ & $0.00 \mathrm{E}+00$ \\
\hline \multirow{2}{*}{ Pstrn } & Example & $3.84 \mathrm{E}+06$ & $3.84 \mathrm{E}+06$ & $3.11 \mathrm{E}+06$ & $1.40 \mathrm{E}+06$ & $2.59 \mathrm{E}+06$ & $0.00 \mathrm{E}+00$ \\
\cline { 2 - 8 } & Application & $3.84 \mathrm{E}+06$ & $3.84 \mathrm{E}+06$ & $3.11 \mathrm{E}+06$ & $1.39 \mathrm{E}+06$ & $2.64 \mathrm{E}+06$ & $0.00 \mathrm{E}+00$ \\
\hline \multirow{2}{*}{ Pstrn } & Example & $2.50 \mathrm{E}+06$ & $2.50 \mathrm{E}+06$ & $2.02 \mathrm{E}+06$ & $9.11 \mathrm{E}+05$ & $1.65 \mathrm{E}+06$ & $0.00 \mathrm{E}+00$ \\
\cline { 2 - 8 } & Application & $2.50 \mathrm{E}+06$ & $2.50 \mathrm{E}+06$ & $2.02 \mathrm{E}+06$ & $9.06 \mathrm{E}+05$ & $1.72 \mathrm{E}+06$ & $0.00 \mathrm{E}+00$ \\
\hline \multirow{2}{*}{ Mn } & Example & $0.00 \mathrm{E}+00$ & $1.35 \mathrm{E}+08$ & $2.17 \mathrm{E}+08$ & $2.83 \mathrm{E}+08$ & $2.42 \mathrm{E}+08$ & $2.07 \mathrm{E}+08$ \\
\cline { 2 - 8 } & Application & $0.00 \mathrm{E}+00$ & $1.34 \mathrm{E}+08$ & $2.17 \mathrm{E}+08$ & $2.82 \mathrm{E}+07$ & $2.42 \mathrm{E}+08$ & $1.95 \mathrm{E}+08$ \\
\hline \multirow{2}{*}{ DMn } & Example & $0.00 \mathrm{E}+00$ & $8.75 \mathrm{E}+07$ & $1.41 \mathrm{E}+08$ & $1.84 \mathrm{E}+08$ & $1.57 \mathrm{E}+08$ & $1.87 \mathrm{E}+08$ \\
\cline { 2 - 8 } & Application & $0.00 \mathrm{E}+00$ & $8.74 \mathrm{E}+07$ & $1.4 \mathrm{E}+08$ & $1.84 \mathrm{E}+08$ & $1.6 \mathrm{E}+08$ & $1.76 \mathrm{E}+08$ \\
\hline \multirow{2}{*}{ Mstrn } & Example & $0.00 \mathrm{E}+00$ & $1.37 \mathrm{E}+08$ & $2.35 \mathrm{E}+08$ & $3.20 \mathrm{E}+08$ & $2.66 \mathrm{E}+08$ & $2.07 \mathrm{E}+08$ \\
\cline { 2 - 8 } & Application & $0.00 \mathrm{E}+00$ & $1.37 \mathrm{E}+08$ & $2.35 \mathrm{E}+08$ & $3.19 \mathrm{E}+08$ & $2.64 \mathrm{E}+08$ & $1.95 \mathrm{E}+08$ \\
\hline \multirow{2}{*}{$\Phi M s t r n$} & Example & $0.00 \mathrm{E}+00$ & $8.88 \mathrm{E}+07$ & $1.53 \mathrm{E}+08$ & $2.08 \mathrm{E}+08$ & $1.73 \mathrm{E}+08$ & $1.87 \mathrm{E}+08$ \\
\cline { 2 - 8 } & Application & $0.00 \mathrm{E}+00$ & $8.87 \mathrm{E}+07$ & $1.53 \mathrm{E}+08$ & $2.08 \mathrm{E}+08$ & $1.72 \mathrm{E}+08$ & $1.76 \mathrm{E}+08$ \\
\hline
\end{tabular}




\section{Chapter 6 - Conclusions and Recommendations}

\subsection{Introduction}

After a thorough review of the design methodology provided in Chapter 13 of Introduction to Composite Materials Design [4], $\mathrm{ACl} 440.2 \mathrm{R}$ [1], and $\mathrm{ACl} 318$ [2], a programming method for implementing the described design procedure for developing an add-on application for Computer Aided Design Environment for Composites (CADEC) was developed and then tested through a Windows Form application to verify the methods outputs. C\# (pronounced "C sharp") and .NET framework were used in the development of the programming method because of the object-oriented programming which creates reusable code.

The class methods were designed to be reused and to be relatively simple. In that way, if an individual wanted to develop an independent application to design and verify strengthening of beams and columns could do so by just using the classes discussed in this document. The class methods were broken down into different members and different steps of the design process to avoid confusion among the classes. With that said some classes are still used in some or all of the design procedures. Also, all naming of variables uses the full proper name of the variable to eliminate any possible confusion of what the class method is requesting. For example, "concreteCompressiveStrength" is the name of the variable for the concrete compressive strength.

To see if the class methods could be used to create an application that produced reasonable results, a Windows Forms application was created. The application used the class methods in a manner similar to the design methodology discussed in Introduction to Composite Material Design [4] where the user has to just select the member type that is being strengthened, provide the requested values, and configure the FRP strengthening reinforcement. The application provides the rest of the process by 
displaying the calculated values and verifies that the strengthened design meets all required capacities and serviceability limitations.

\subsection{Comparison of Outputs against Examples}

Several examples were run through the application to produce results and then compared to hand solved examples shown in Appendix A. It must be noted that the examples used had to be updated to meet the current $\mathrm{ACl} 440$ requirements. Examples included beams needing strengthening for flexure, shear; columns needing strengthening for pure axial loading; and combination of axial and bending loading.

The majority of the outputs produced were either identical values or values that were extremely close to the hand-solved examples with errors of less than $0.02 \%$. That minor difference can be account for because the class methods and the application all use double float precision variables which provide values up to the $16^{\text {th }}$ decimal place. Therefore, resulting small differences attributed to rounding is to be expected and acceptable.

The only major discrepancies of significance were in calculating critical points of the interaction diagram. Point $E$, where the eccentricity was fixed to produce a 45 degree line on the interaction diagram, has noticeable differences. This can be attributed to how the point is calculated. To find Point E, an iterative process is used to determine the neutral axis that would produce the fixed value. Each time the error was calculated. The class method for calculating Point E does this by implementing a while-loop based on the error. The loop will continue to adjust the neutral axis until the error is less than a hundredth which results in a more accuratly and consistency than the hand-solved examples, which often only do a few iterations to reach an acceptable error which typically can be around $1 \%$.

\subsection{Classes and Forms Redundancies}


Though the methods and Windows Form application does work and produces accurate outputs, the programming is often redundant. Several classes often calculate the same values but are separated because of the organization of the classes. Also, in some classes, methods often calculate the same value internally, which is inefficient. Most of the class redundancies are often caused by the lack of foresight in the development and can easily be remedied creating new class methods just for those particular calculations and by consolidating repeated processes into few classes. The application forms experience similar issues because of the lack of forethought. Such redundancies occur on property forms. Length and moment of inertia are not relevant to the shear design but appear on the beam shear properties form. Length also appears on columns and beam-column paths and is also not needed.

\subsection{Windows Form Application Errors}

The application is still prone to errors caused by user interaction. These errors are not calculation errors, but rather input errors. Though, the forms do try to prevent majority of these errors by validating the inputs, but when the user has a choice in items, the form will often fail to address or address the problem because not every potential outcome has been addressed in the validation process. The most obvious case of this is in the beam-column properties form when entering the distances and areas of the steel reinforcement. If the user inputs a wrong number, the user will never be able to change that number unless by starting the application from the beginning. Also, If the user inputs the wrong number in one of the textboxes before entering the second textbox, the user will also be unable to fix it because once the focus is left from one of the textboxes after the initial value has been entered, the application will either think there is an invalid input, or it will take the value and add it to the arrays.

\subsection{Recommendations}

Though the class methods and the application do work for beams and columns, several changes could be made to improve the performance and appearance to the application. The majority of the 
design process occurs in the forms, but this can also be down in the classes and would eliminate any potential for errors and confusions in the development of other applications for strengthening beams and columns using FRP. This would not prevent a different design approach being used along with the classes, because any required class method that is needed can be called and used in any manner that is required.

The application could be more user-friendly and more pleasing. Though the forms are simple and straightforward, the user must be familiar with the application prior to using it to make the process run smoothly. Also, restricting the user inputs by using more dropdown lists that provide common values for such items such as yield strength of the steel would reduce the amount of validation needed on user inputs. The results also could be displayed in a not so dull format and an option to see all internally calculated values should be an option. Another addition to the application would be to add diagrams on the forms to help demonstrate the wrapping scheme being chosen. The shear properties form could benefit from having a simple diagram that changes depending on the selection of the wrapping scheme. The diagram would simple be a cross-section displaying the shape of the beam and the locations the FRP were to be applied. A diagram of this nature could benefit each design path. An adaptive diagram that would create the cross-section described by the properties form would be a huge addition as well, and it would allow the user to see if the application is interpreting the original concrete structure as expected.

Lastly, $\mathrm{ACl} 440.2 \mathrm{R}-08$ does provide guidelines for strengthening of prestressed reinforced concrete beams, but was not considered in the development of these class methods because it was not discussed in the Chapter 13 of Introduction to Composite Materials Design [4] and didn't correlate with CADEC's objective. Adding prestressed concrete beams to the class methods could easily be done and would add an extra dimension to the add-on for CADEC. 


\section{References}

[1] ACl 440.2R-08. Guide for the Design and Construction of Externally Bonded FRP Systems for Strengthening Concrete Structures. Farmington Hills, MI: American Concrete Institute, 2008. Print.

[2] ACl 318-08. Building Code Requirements for Structural Concrete and Commentary (318R-08). Farmington Hills, MI: American Concrete Institute, 2008

[3] Bank, Lawrence. Composites Construction: Structural Design with FRP Materials. Hoboken, NJ: John Wiley \& Sons, 2006. Print.

[4] Barbero, Ever. Introduction to Composite Materials Design. 2nd Ed. Boca Raton, FL: 2011. Print.

[5] GangaRao, Hota, Narendra Taly, and P.V. Vijay.Reinforced Concrete Design with FRP Composites. Boca Raton, FL: Taylor \& Francis Group, 2007. Print.

[6] Nawy, Edward. Reinforced Concrete A Fundamental Approach. 6th Ed. Upper Saddle River, NJ: Pearson Education, 2009. Print. 


\section{Appendix A: Updated Examples}




\section{Example A}

Geometric propettes

$l:=6300$

$b:=300$

$h:=600$

dearConer: $=40$

$d:=h-$ clearCover $=560$

$I:=\frac{b \cdot h}{12}=5.4 \cdot 10^{9}$

Loading requirements

$L L:=10.5 \cdot 1.6=16.8$

$D L:=13.5$

$w_{u}=1.2 \cdot D L+1.6 \cdot L L=43.08$

$M_{c i}:=\frac{w w_{i s} \cdot l}{8}=2.137 \cdot 10^{8}$ steel reinforcement

$A_{s}:=850$

$f_{y}:=413.68$

$E_{s}: 200000$

concrete

$f_{c}^{\prime}:=34.5$

$E_{c}:=4700 \sqrt{f_{Q}^{\prime}}=2.761 \cdot 10^{4}$
FRP

$f_{f}^{\prime}=2200$

$\varepsilon_{f t u t}^{\prime}:=0.011$

$t_{f}:=0.333$

carbon

interior exposure

Step 1: Initial Moment capacity and max. allowable reinforcement.

$\beta_{1}:=0.85-05 \cdot \frac{\left(f_{e}^{\prime}-27.57\right)}{6.9}=0.8$

$c_{0}:=\frac{A_{s} \cdot f_{y}}{.85 \cdot f^{\prime} \cdot \beta_{1} \cdot b}=49975$

$M_{0}:=A_{s} \cdot f_{3} \cdot\left(d-\frac{c_{0} \cdot \beta_{1}}{2}\right)=1.899 \cdot 10^{8}$

$\phi M_{s}=0.9 \cdot M_{o}=1.709 \cdot 10^{\circ}$

check minimum loading capacity:

$$
S_{\text {uhsost }}:=1.1 \cdot M_{D L}+.75 \cdot M_{L L}=1.362 \cdot 10^{8}
$$

$$
\phi M_{0} \geq S_{\text {thostr }} \rightarrow 1 \quad \text { true }
$$

check maximum allowable strengthening:

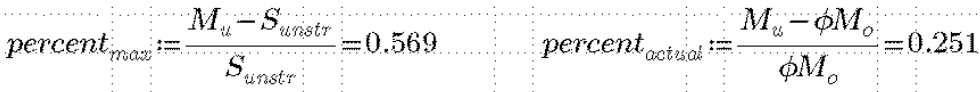

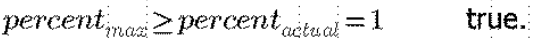


Step 2: Determine the initial strain at the soffit of the beam

$C_{1}:=E_{c} \cdot \frac{b}{2}=4.141 \cdot 10^{5}$

$C_{2}:=E_{s}: A_{s}=1.7 \cdot 10^{8}$

$C_{3}:=-E_{s} \cdot A_{\dot{s}} \cdot d=-9.52 \cdot 10^{10}$

$C_{2}{ }^{2}-4 \cdot C_{1} \cdot C_{3}=1.606 \cdot 10^{18}$

$q_{1}:=\frac{-C_{2}+\sqrt{C_{2}^{3}-4 \cdot C_{1} \cdot C_{3}}}{2 \cdot C_{1}}=132.481$

$q_{2}=\frac{-C_{2}-\sqrt{C_{2}^{2}-4 \cdot C_{1} \cdot C_{3}}}{2 \cdot C_{1}}=-173.534$

$c_{A}:=q_{1}=132.481$

$E I_{c r}:=\frac{E_{c} \cdot b \cdot c_{A}^{3}}{3}+E_{s} \cdot A_{s} \cdot\left(d-c_{A}\right)^{2}=3.749 \cdot 10^{13}$

$\varepsilon_{b z}:=\frac{M_{D L} \cdot\left(h-c_{A}\right)}{E I_{c r}}=8.352 \cdot 10^{-4}$

Step 3: FRP system

$C_{E}:=0.95 \quad$ carbon and interior exposure

$f_{f t u}:=C_{E} \cdot f_{f}^{\prime}=2.09 \cdot 10^{3}$

$\epsilon_{i v}:=C_{E} \cdot c_{f u}^{\prime}=0.01$

$E_{f}:=\frac{f_{f u}}{\varepsilon_{f x}}=2 \cdot 10^{z}$

Step 4: Design FRP reinforcment

$n:=1 \quad$ assume 1 layer for initial calculations

$\varepsilon_{f l}:=0.41 \cdot \sqrt{\frac{f_{c}^{\prime}}{\left(n \cdot E_{i} \cdot t_{f}\right)}}=0.009$ 


$$
\begin{aligned}
& \varepsilon_{f \in}:=\text { if } \varepsilon_{f a}<0.9 \cdot \varepsilon_{f u} \\
& \begin{array}{l}
\| \varepsilon_{f a} \\
\| 0.9 \cdot \varepsilon_{f i n}
\end{array} \\
& \varepsilon_{f e}=0.009 \\
& \psi_{1}:=0.85 \\
& c_{b}=\frac{.003 \cdot h}{.003+\varepsilon_{f e}+\varepsilon_{3 i}}=136.707 \\
& \varepsilon_{s}=\frac{003}{c_{b}}\left(d-c_{b}\right)=0.009
\end{aligned}
$$

calculate the area required for $B S C$ depending if the steel yeilded:

$$
\begin{aligned}
& A_{f_{s}}:=\text { if } \varepsilon_{s}>\frac{f_{u}}{E_{s}} \\
& \| \frac{\left(0.85 \cdot f_{b}^{j} \cdot \beta_{1} \cdot b \cdot c_{b}-A_{w} \cdot f_{i}\right)}{E_{f} \cdot \varepsilon_{f e}} \\
& \text { else } \\
& \| \frac{\left(0.85 \cdot f_{c}^{\prime} \cdot \beta_{1} \cdot b \cdot c_{b}-A_{3} \cdot E_{5} \cdot \varepsilon_{5}\right)}{E_{f} \cdot \varepsilon_{f e}} \mid
\end{aligned}
$$

$A_{f b}=326.983$

determine the number of layers assuming full base of the beam:

$n=\left(\frac{A_{f b}}{\left(t_{\vec{f}} \cdot b\right)}\right)=3.273$

Step 5: configure FRP reinforcement

$$
n:=1 \quad b_{f}:=200 \quad A_{f}:=n \cdot b_{f} \cdot t_{f}=66.6
$$


Step 6: calculate new moment capacity

$\varepsilon_{f d}:=0.41 \cdot \sqrt{\frac{f_{b}^{\prime}}{\left(n \cdot E_{f}^{\prime}\right.} \cdot t_{f)}}=0.009$

$\varepsilon_{f e}:=$ if $\varepsilon_{f d l}<0.9 \cdot \varepsilon_{f u} \quad \varepsilon_{f e}=0.009$

$\| \operatorname{lic}_{\mathrm{f}}$

$\| 0.9 \cdot c_{j u}$

$A_{f}<A_{f b}=1 \quad$ false, therefore WSC design

assume steel yeilds:

$c:=\frac{A_{s} \cdot f_{y}+A_{f} \cdot E_{j} \cdot c_{f}}{0.85 \cdot f_{c}^{\prime} \cdot \beta_{1} \cdot b}=67.641$

$\varepsilon_{s}=\frac{003}{c}(d-c)=0.022 \quad \varepsilon_{s}>005=1 \quad$ tension controlled state

$M_{\mathrm{y}}:=A_{s} \cdot f_{y} \cdot\left(d-\beta_{1} \cdot \frac{c}{2}\right)+.85 \cdot A_{f} \cdot E_{f} \cdot \varepsilon_{f e} \cdot\left(h-\beta_{1} \cdot \frac{c}{2}\right)=2.479 \cdot 10^{8}$

$\phi: 0.9$ because of tension controlled state $\phi M_{n}=\phi \cdot M_{r_{0}}=2.231 \cdot 10^{8}$

$\phi M_{n}>M_{0}=1 \quad$ meets required loading capacity. 
Step 7: check serviceability requirements

$C_{1}:=E_{c} \cdot \frac{b}{2}=4.141 \cdot 10^{\hbar}$

$C_{2}:=E_{s} \cdot A_{s}+E_{j} \cdot A_{f}=1.833 \cdot 10^{8}$

$C_{j}:=-E_{s} \cdot A_{s} * d-E_{f} \cdot A_{f} \cdot h=-1.032 \cdot 10^{11}$ $q_{1}:=\frac{-C_{2}+\sqrt{C_{2}^{2}-4 \cdot C_{1} \cdot C_{3}}}{2 \cdot C_{1} \quad \vdots \quad}=137.27$

$q_{2}:=\frac{-C_{2}-\sqrt{{C_{2}{ }^{2}-4 \cdot C_{1} \cdot C_{3}}_{2}}}{2 \cdot C_{1}}=-181.54$

$c_{B}:=q_{1}=137.27$

neutral axis of stage $B$ of serviceability requirements

concrete stress

$f_{c S t \cos A}^{f}=\frac{E_{c} \cdot\left(M_{D L} \cdot c_{A}\right)}{E_{c} \cdot b \cdot \frac{c_{A}}{3}+E_{s} \cdot A_{s} \cdot\left(d-c_{A}\right)^{2}}=6.534$

$f_{\text {stateB }}:=\frac{E_{c} \cdot\left(M_{L_{F}} \cdot c_{B}\right)}{E_{c} \cdot b \cdot \frac{c_{B}^{3}}{3}+E_{v} \cdot A_{g} \cdot\left(d-c_{B}\right)^{2}+E_{f} \cdot A_{f} \cdot\left(h-c_{B}\right)^{2}}=7.824$

$f_{c}:=f_{C S t a g e A}+f_{C S \operatorname{tag} e \theta}=14.357$

$f_{s} \leq 0.45 \cdot f_{c}^{\prime}=1 \quad$ does not meet stress limits for concrete.

steel stress

$f_{s \text { scog }}:=\frac{E_{s} \cdot\left(M_{D L} \cdot\left(d-c_{A}\right)\right)}{c_{A}^{3}+E_{s} \cdot A_{s} \cdot\left(d-c_{A}\right)^{2}}=152.753$

$f_{s \delta \text { tage } E}:=\frac{E_{s} \cdot\left(M_{L L} \cdot\left(d-c_{B}\right)\right)}{E_{\mathrm{c}} \cdot b \cdot \frac{c_{B}^{3}}{3}+E_{s} \cdot A_{s} \cdot\left(d-c_{B}\right)^{2}+E_{f} \cdot A_{f} \cdot\left(h-c_{B}\right)^{8}}=174.548$

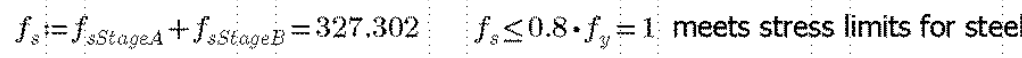


FRP stress

$f_{f}=f_{s} \cdot\left(\frac{E_{f}}{E_{s}}\right) \cdot \frac{\left(h-c_{D}\right)}{\left(d-\varepsilon_{B}\right)}-\varepsilon_{b i} \cdot E_{f}=191.227$

$f_{f} \leq 0.55 \cdot f_{f u}=1 \quad$ meets stress limits for glass FRP 


\section{Example B}

Geometric properties

$l:=9140$

$b:=305$

$h:=660$

dearConer $:=77$

$d:=h-$ dearCover $=583$

$I:=\frac{b \cdot h}{12}=7.307 \cdot 10^{9}$

Loading requirements

$L L:=24.1 \cdot 1.5=36.15$

$D L:=4.743$

$w_{t}=1.2 \cdot D L+1.6 \cdot L L=63.532$

$M_{u}:=\frac{w_{2} \cdot l}{8}=6.634 \cdot 10^{8}$

Step 1: Initial Moment capacity and max, allowable reinforcement.

$\beta_{1}:=0.85$

$c_{0}:=\frac{A_{s} \cdot f_{y}}{85 \cdot f^{\prime} \cdot \beta_{1} \cdot b}=167,315$

$M_{0}:=A_{s} \cdot f_{3} \cdot\left(d-\frac{c_{0} \cdot \beta_{1}}{2}\right)=5.209 \cdot 10^{8}$

$\phi M_{o}:=0.9 \cdot M_{s}=4.688 \cdot 10^{8}$

check minimum loading capacity:

$$
S_{\text {uhastr }}:=1.1 \cdot M_{D L}+.75 \cdot M_{L L}=3.376 \cdot 10^{8}
$$

$$
\phi M_{0} \geq S_{t h k s t r} \rightarrow 1 \quad \text { true }
$$

check maximum allowable strengthening:

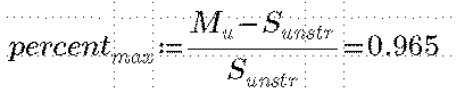

percent $t_{\text {ma }} \geq$ percent atwal $=1$

true.

$M_{D L}=\frac{D L \cdot l^{2}}{8}=4.953 \cdot 10^{7}$
FRP

$f_{f}^{\prime}:=1020$

$\varepsilon_{f_{t h}^{\prime}}^{\prime}:=0.0129$

$t_{f}:=0.144$

glass-expoxy

exterior exposure

$f^{\prime}:=27.6$

$E_{c}:=4700 \sqrt{f_{c}^{\prime}}=2.469 \cdot 10^{4}$

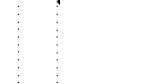


Step 2: Determine the initial strain at the soffit of the beam

$C_{1}:=E_{c} \cdot \frac{b}{2}=3.765 \cdot 10^{5}$

$C_{2}:=E_{s}: A_{s}=4,916 \cdot 10^{8}$

$C_{3}:=-E_{s}^{*} \cdot A_{s} \cdot d=-2.866 \cdot 10^{11}$

$C_{2}{ }^{2}-4 \cdot C_{1} \cdot C_{3}=4.558 \cdot 10^{18}$

$q_{1}:=\frac{-C_{2}+\sqrt{C_{2}^{3}-4 \cdot C_{1} \cdot C_{3}}}{2 \cdot C_{1}}=218.226$

$q_{2}=\frac{-C_{2}-\sqrt{C_{2}^{2}-4 \cdot C_{1} \cdot C_{3}}}{2 \cdot C_{1}}=-348.78$

$c_{A}:=q_{1}=218.226$

$E I_{c r}:=\frac{E_{c} \cdot b \cdot c_{A}{ }^{3}}{3}+E_{s} \cdot A_{s} \cdot\left(d-c_{A}\right)^{2}=9.15 \cdot 10^{13}$

$\varepsilon_{b i}:=\frac{M_{D L} \cdot\left(h-c_{A}\right)}{E I_{G Y}}=2.391 \cdot 10^{-4}$

Step 3: FRP system

$C_{E}:=0.65 \quad$ glass and exterior exposure

$f_{f u t}:=C_{k p} \cdot f_{f}^{\prime}=663$

$\varepsilon_{j u}:=C_{E} \cdot \epsilon_{j u}^{\prime}=0.008$

$E_{f}:=\frac{f_{\text {fu }}}{\varepsilon_{\text {fui }}}=7.907 \cdot 10^{4}$

Step 4: Design FRP reinforcment

$n:=1 \quad$ assume 1 layer for initial calculations

$\varepsilon_{f d}:=0.41 \cdot \sqrt{\frac{f_{c}^{\prime}}{\left(n \cdot E_{i} \cdot t_{f}\right)}}=0.02$ 


$$
\begin{aligned}
& \varepsilon_{f \in}:=\text { if } \varepsilon_{f a}<0.9 \cdot \varepsilon_{f u} \\
& \begin{array}{l}
\| \varepsilon_{f d} \\
\text { else } \\
\| 0.9 \cdot \varepsilon_{f \neq i}
\end{array} \\
& \varepsilon_{f e}=0.008 \\
& f_{f}:=0.85 \\
& c_{b}=\frac{.003 \cdot h}{.003+\varepsilon_{f \varepsilon}+\varepsilon_{b i}}=183.578 \\
& \varepsilon_{s}=\frac{003}{c_{b}}\left(d-c_{b}\right)=0.007
\end{aligned}
$$

calculate the area required for BSC depending if the steel yeilded:

$$
\begin{aligned}
& A_{f_{s}}:=\text { if } \varepsilon_{s}>\frac{f_{u}}{E_{s}}
\end{aligned}
$$

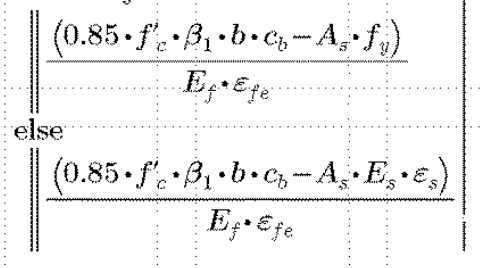

$A_{f b}=165,758$

determine the number of layers: assuming full base of the beam

$n=\left(\frac{A_{f b}}{\left(t_{\vec{f}} \cdot b\right)}\right)=3.774$

Step 5: configure FRP reinforcement

$$
n=5 \quad b_{f}=300 \quad A_{f}=n \cdot b_{f} \cdot t_{f}=216
$$

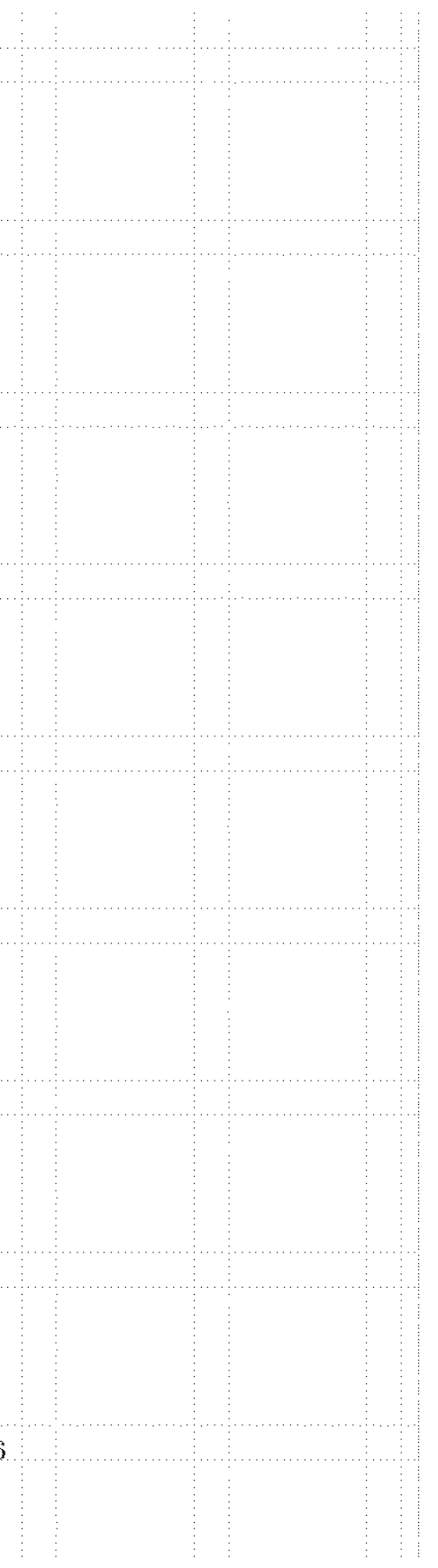


Step 6: calculate new moment capacity

$\varepsilon_{f d}:=0.41 \cdot \sqrt{\left.\frac{f_{b}^{\prime}}{\left(n \cdot E_{f}\right.} \cdot t_{f}\right)}=0.009$

$\varepsilon_{f e}=$ if $\varepsilon_{f i l}<0.9 \cdot \varepsilon_{q u} \quad \varepsilon_{f e}=0.008$

$$
\begin{aligned}
& \| s_{y d} \\
& \text { else } \\
& \| 0.9 \cdot \sigma_{j u}
\end{aligned}
$$

$A_{f}<A_{f 0}=0 \quad$ false, therefore SSC design

assume steel yeilds:

$C_{1}:=0.85 \cdot f_{c}^{\prime} \cdot \beta_{1} \cdot b=6.082 \cdot 10^{3}$

$C_{2}:=E_{f} \cdot A_{f} \cdot\left(\varepsilon_{f e}+\varepsilon_{b i}\right)-A_{v} \cdot f_{t}=-8.846 \cdot 10^{5}$

$C_{3}=-h \cdot E_{f} \cdot A_{j} \cdot 003=-3.382 \cdot 10^{7}$

$q_{1}=\frac{-C_{2}+\sqrt{Q_{2}^{2 y}-4 \cdot C_{1} \cdot C_{3}}}{2 \cdot C_{1}}=176.885$

$q_{2}: \frac{-C_{2}-\sqrt{{O_{2}^{2}}^{2}-4 \cdot C_{1} \cdot C_{3}}}{2 \cdot C_{1}}=-31.433$

$c:=q_{1}=176.885$

$\varepsilon_{s}=\frac{003}{c}(d-c)=0.007$

$\varepsilon_{s}>.005=1$ tension controlled state

$M_{\gamma b}=A_{\varepsilon} \cdot f_{y} \cdot\left(d-\beta_{1} \cdot \frac{c}{2}\right)+.85 \cdot A_{f} \cdot E_{f} \cdot \varepsilon_{f e^{*}} \cdot\left(h-\beta_{1} \cdot \frac{c}{2}\right)=5.808 \cdot 10^{8}$

$\phi:=0.9$ because of tension controlled state $\phi M_{p}:=\phi \cdot M_{n}=5.228 \cdot 10^{8}$

$\phi M_{n}>M_{x}=0 \quad$ does not meet required loading capacity. 
Step 7: check serviceability requirements

$C_{1}:=E_{c} \cdot \frac{b}{2}=3.765 \cdot 10^{6}$

$C_{2}:=E_{s} \cdot A_{s}+E_{f} \cdot A_{f}=5.087 \cdot 10^{8}$

$C_{j}:=-E_{s} \cdot A_{s} \cdot d-E_{f} \cdot A_{f} \cdot h=-2.979 \cdot 10^{11}$

$q_{1}=\frac{-C_{2}+\sqrt{C_{2}^{25}-4 \cdot C_{1} \cdot C_{3}}}{2 \cdot C_{1}}=221.711$

$q_{2}:=\frac{-C_{2}-\sqrt{O_{2}^{2}-4 \cdot C_{1} \cdot C_{3}}}{2 \cdot C_{1}}=-356.8$

$c_{B}:=q_{1}=221.711$

neutral axis of stage $B$ of serviceability requirements

concrete stress

$f_{c S t \cos t}=\frac{E_{c} \cdot\left(M_{D L} \cdot c_{A}\right)}{c_{A}}=2.917$

$f_{\text {stateB }}:=\frac{E_{c} \cdot\left(M_{L Z} \cdot c_{B}\right)}{E_{c} \cdot b \cdot \frac{c_{B}^{3}}{3}+E_{*} \cdot A_{s} \cdot\left(d-c_{B}\right)^{2}+E_{f} \cdot A_{f} \cdot\left(h-c_{B}\right)^{2}}=21.797$

$f_{c}:=f_{C S t \operatorname{cog} \mathrm{A} A}+f_{\operatorname{cotag} \mathrm{tag} E}=24.714$

$f_{c} \leq 0.45 \cdot f^{\prime}=0 \quad$ does not meet stress limits for concrete.

steel stress

$f_{s \text { sogeA }}:=\frac{E_{s} \cdot\left(M_{D L} \cdot\left(d-c_{A}\right)\right)}{c_{A}^{3}+E_{s} \cdot A_{s} \cdot\left(d-c_{A}\right)^{2}}=39.49$

$f_{s 8 \text { tage } B}:=\frac{E_{s} \cdot\left(M_{L L} \cdot\left(d-c_{B}\right)\right)}{E_{c} \cdot b \cdot \frac{c_{B}^{3}}{3}+E_{s} \cdot A_{s} \cdot\left(d-c_{B}\right)^{2}+E_{f} \cdot A \cdot\left(h-c_{B}\right)^{3}}=287.708$

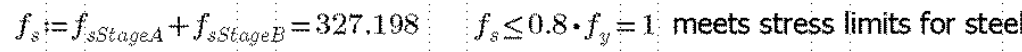


FRP stress

$f_{f}:=f_{s} \cdot\left(\frac{E_{f}}{E_{s}}\right) \cdot \frac{\left(h-c_{D}\right)}{\left(d-a_{D}\right)}-\varepsilon_{b i} \cdot E_{f}=138.019$

$f_{f} \leq 0.2 \cdot f_{f^{\prime} \omega}=0 \quad$ meets stress limits for glass FRP 


\section{Example C}

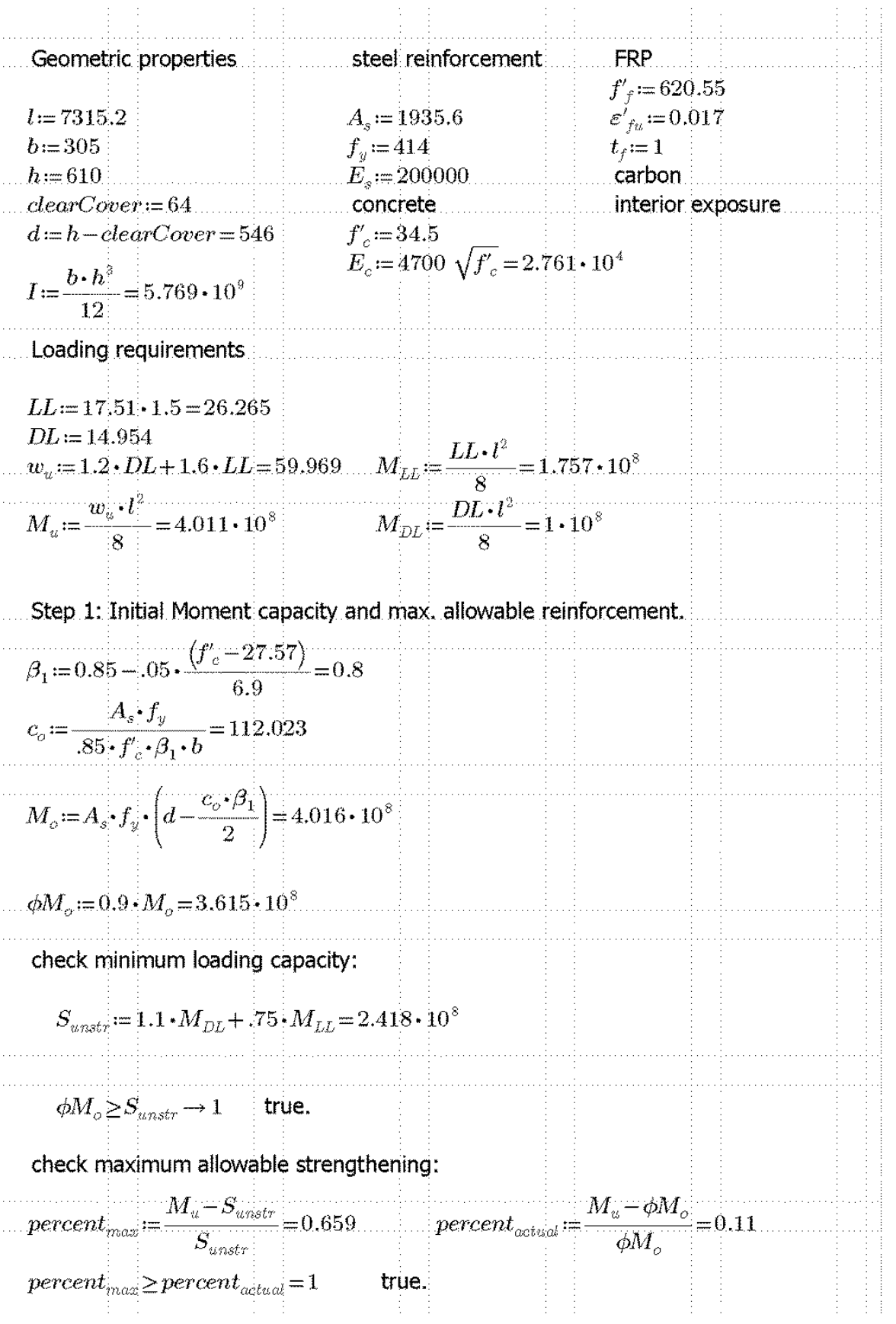


Step 2: Determine the initial strain at the soffit of the beam

$C_{1}:=E_{c} \cdot \frac{b}{2}=4.21 \cdot 10^{6}$

$C_{2}:=E_{s}: A_{s}=3.871 \cdot 10^{8}$

$C_{3}:=-E_{s} \cdot A_{3} \cdot d=-2.114 \cdot 10^{11}$

$C_{2}{ }^{2}-4 \cdot C_{1} \cdot C_{3}=3.709 \cdot 10^{18}$

$q_{1}=\frac{-C_{2}+\sqrt{C_{2}^{2}-4 \cdot C_{1} \cdot C_{3}}}{2 \cdot C_{1}}=182.76$

$q_{2}=\frac{-C_{2}-\sqrt{C_{2}^{2}-4 \cdot C_{1} \cdot C_{3}}}{2 \cdot C_{1}}=-274.713$

$c_{A}:=q_{1}=182.76$

$E I_{c r}:=\frac{E_{c} \cdot b \cdot c_{A}^{3}}{3}+E_{s} \cdot A_{s} \cdot\left(d-c_{A}\right)^{2}=6.821 \cdot 10^{12}$

$\varepsilon_{b i}:=\frac{M_{D L} \cdot\left(h-c_{A}\right)}{E I_{G Y}}=6.265 \cdot 10^{-4}$

Step 3: FRP system

$C_{E}:=0.95 \quad$ carbon and interior exposure

$f_{f t}:=C_{p i} \cdot f_{f}^{\prime}=589.523$

$\varepsilon_{j u}:=C_{E} \cdot \epsilon_{j u}^{\prime}=0,016$

$E_{f}:=\frac{f_{f u}}{\varepsilon_{f, x}}=3.65 \cdot 10^{4}$

Step 4: Design FRP reinforcment

$n:=1 \quad$ assume 1 layer for initial calculations

$\varepsilon_{f d}:=0.41 \cdot \sqrt{\frac{f_{c}^{z}}{\left(n \cdot \bar{E}_{f} \cdot t_{f}\right)}}=0.013$ 


$$
\begin{aligned}
& \varepsilon_{f \in}:=\text { if } \varepsilon_{f a}<0.9 \cdot \varepsilon_{f u} \\
& \begin{array}{l}
\| \varepsilon_{f d} \\
\| l s e \\
\| 0.9 \cdot \varepsilon_{f \neq i}
\end{array} \\
& \varepsilon_{f e}=0.013 \\
& \psi_{y}:=0.85 \\
& c_{b}=\frac{.003 \cdot h}{.003+\varepsilon_{f \varepsilon}+\varepsilon_{b i}}=112.746 \\
& \varepsilon_{s}=\frac{003}{c_{b}}\left(d-c_{b}\right)=0.012
\end{aligned}
$$

calculate the area required for BSC depending if the steel yeilded:

$$
\begin{aligned}
& A_{f_{s}}:=\text { if } \varepsilon_{s}>\frac{f_{u}}{E_{s}}
\end{aligned}
$$

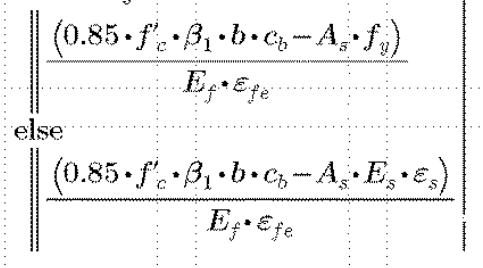

$A_{f b}=11,249$

determine the number of layers: assuming full base of the beam:

$n=\left(\frac{A_{f b}}{\left(t_{\vec{f}} \cdot b\right)}\right)=0.037$

Step 5: configure FRP reinforcement

$n=2 \quad b_{f}=305$

$$
A_{f}:=n \cdot b_{f} \cdot t_{f}=610
$$


Step 6: calculate new moment capacity

$\varepsilon_{f d}:=0.41 \cdot \sqrt{\left.\frac{f_{b}^{\prime}}{\left(n \cdot E_{f}\right.} \cdot t_{f}\right)}=0.009$

$\varepsilon_{f e}=$ if $\varepsilon_{f l l}<0.9 \cdot \varepsilon_{\gamma} \quad \varepsilon_{f e}=0.009$

$$
\begin{aligned}
& \| s_{y d} \\
& \text { else } \\
& \| 0.9 \cdot \sigma_{j u}
\end{aligned}
$$

$A_{f}<A_{f o}=0 \quad$ false, therefore WSC design

assume steel yeilds:

$C_{1}:=0.85 \cdot f_{c}^{\prime} \cdot \beta_{1} \cdot b=7.153 \cdot 10^{3}$

$C_{2}:=E_{f}: A_{f} \cdot\left(\varepsilon_{f e}+\varepsilon_{3 i}\right)-A_{s} \cdot f_{u}=-5.889 \cdot 10^{5}$

$C_{3}:=-h \cdot E_{f} \cdot A_{f} \cdot 003=-4.075 \cdot 10^{7}$

$q_{1}=\frac{-C_{2}+\sqrt{C_{2}^{2}-4 \cdot C_{1} \cdot C_{3}}}{2 \cdot C_{1}}=127.135$

$q_{2}: \frac{-C_{2}-\sqrt{C_{2}^{2}-4 \cdot C_{1} \cdot C_{3}}}{2 \cdot C_{1}}=-44.806$

$c:=q_{1}=127.135$

$\varepsilon_{s}:=\frac{003}{e}(d-c)=0.01$

$e_{s}>.005=1 \quad$ tension controlled state

$M_{n}:=A_{s} \cdot f_{g} \cdot\left(d-\beta_{1} \cdot \frac{c}{2}\right)+.85 \cdot A_{f} \cdot E_{f \cdot} \cdot \varepsilon_{f \varepsilon} \cdot\left(h-\beta_{1} \cdot \frac{c}{2}\right)=4,911 \cdot 10^{8}$

$\phi=0.9$ because of tension controlled state

$\phi M_{n}=\phi \cdot M_{n}=4.42 \cdot 10^{8}$

$\phi M_{n}>M_{k}=1 \quad$ meets required loading capacity. 
Step 7: check serviceability requirements

$C_{1}:=E_{c} \cdot \frac{b}{2}=4.21 \cdot 10^{6}$

$C_{2}:=E_{s} \cdot A_{3}+E_{f} \cdot A_{f}=4.094 \cdot 10^{8}$

$C_{3}:=-E_{s} \cdot A_{s} \cdot d-E_{f} \cdot A_{f} \cdot h=-2.25 \cdot 10^{11}$

$q_{1}:=\frac{-C_{2}+\sqrt{C_{2}^{2}-4 \cdot C_{1} \cdot C_{3}}}{2 \cdot C_{1}}=187.593$

$q_{2}:=\frac{-O_{2}-\sqrt{O_{2}{ }^{2}-4 \cdot C_{1} \cdot C_{3}}}{2 \cdot C_{1}}=-284.835$

$c_{B}:=q_{1}=187.593$

neutral axis of stage $B$ of serviceability requirements

concrete stress

$f_{c S t a g A}=\frac{E_{c} \cdot\left(M_{D L} \cdot c_{A}\right)}{c_{A}}+E_{s} \cdot A_{s} \cdot\left(d-c_{A}\right)^{2}=7.399$

$f_{c s \text { ateB }}:=\frac{E_{c} \cdot\left(M_{L, L} \cdot c_{B}\right)}{E_{c} \cdot b \cdot \frac{c_{B}^{3}}{3}+E_{*} \cdot A_{s} \cdot\left(d-c_{B}\right)^{2}+E_{f} \cdot A_{f} \cdot\left(h-c_{B}\right)^{2}}=12.597$

$f_{c}:=f_{C S t a g e A}+f_{C B \operatorname{tag} e}=19.995$

$f_{s} \leq 0.45 \cdot f_{c}^{\prime}=0 \quad$ does not meet stress limits for concrete.

steel stress

$f_{s \text { scog }}:=\frac{E_{s} \cdot\left(M_{D L} \cdot\left(d-c_{A}\right)\right)}{c_{A}^{3}+E_{s} \cdot A_{s} \cdot\left(d-c_{A}\right)^{2}}=106.535$

$f_{\text {sqtage } B}:=\frac{E_{s} \cdot\left(M_{L L} \cdot\left(d-c_{B}\right)\right)}{E_{c} \cdot b \cdot \frac{c_{B}^{3}}{3}+E_{s} \cdot A_{s} \cdot\left(d-c_{B}\right)^{2}+E_{f} \cdot A \cdot\left(h-c_{B}\right)^{2}}=174.355$

$f_{s}=f_{S S t a g e d}+f_{s \operatorname{stg} \theta}=280.89 \quad f_{s} \leq 0.8 \cdot f_{3}=1$ meets stress limits for steel 
FRP stress

$f_{f}:=f_{s} \cdot\left(\frac{E_{f}}{E_{s}}\right) \cdot \frac{\left(n-c_{B}\right)}{\left(d-\epsilon_{B}\right)}-\varepsilon_{b v} \cdot E_{j}=37.551$

$f_{f} \leq 0.55 \cdot f_{f u}=1 \quad$ meets stress limits for glass FRP 


\section{Example D}

Geometric properties

$l:=6700$

$b:=381$

$h:=610$

clearCover: $=64$

$d:=h-$ clearCover $=546$

$I:=\frac{b \cdot h}{12}=7.207 \cdot 10^{9}$

Loading requirements.

$L L:=18.24 \cdot 2=36.48$

$D L:=16.05$

$w_{u}=1.2 \cdot D L+1.6 \cdot L L=77.628$

$M_{u}:=\frac{w_{2} \cdot l}{8}=4.356 \cdot 10^{8}$

Step 1: Initial Moment capacity and max. allowable reinforcement.

$\beta_{1}:=0.85-05 \cdot \frac{\left(f_{e}-27.57\right)}{6.9}=0.774$

$c_{0}:=\frac{A_{s} \cdot f_{y}}{.85 \cdot f^{\prime} \cdot \beta_{1} \cdot b}=88,047$

$M_{0}:=A_{s} \cdot f_{3} \cdot\left(d-\frac{c_{0} \cdot \beta_{1}}{2}\right)=4.295 \cdot 10^{8}$

$\phi M_{s}=0.9 \cdot M_{o}=3.866 \cdot 10^{\circ}$

check minimum loading capacity:

$$
\begin{aligned}
& S_{u, R=t r}=1.1 \cdot M_{D L}+.75 \cdot M_{I L}=2.526 \cdot 10^{8} \\
& \phi M_{o} \geq S_{\text {thRstr }} \rightarrow 1 \quad \text { true. }
\end{aligned}
$$

check maximum allowable strengthening:

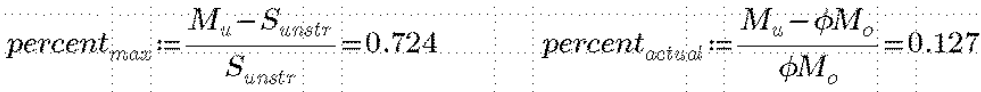

$$
\begin{aligned}
& \text { percent } \geq \text { percent } t_{\text {actual }}=1 \quad \text { true. }
\end{aligned}
$$

FRP

$f_{f}^{\prime}:=650$

interior exposure

$f_{c}^{\prime}:=38$

$M_{L E}=\frac{L L \cdot l^{2}}{8}=2.047 \cdot 10^{8}$

$M_{D L}=\frac{D L \cdot l^{2}}{8}=9.006 \cdot 10^{7}$ 
Step 2: Determine the initial strain at the soffit of the beam

$C_{1}:=E_{c} \cdot \frac{b}{2}=5.519 \cdot 10^{5}$

$C_{2}:=E_{s}: A_{s}=4.054 \cdot 10^{8}$

$C_{3}:=-E_{s} \cdot A_{s} \cdot d=-2.213 \cdot 10^{11}$

$C_{2}{ }^{2}-4 \cdot C_{1} \cdot C_{3}=5.051 \cdot 10^{18}$

$q_{1}:=\frac{-C_{2}+\sqrt{C_{2}^{2}-4 \cdot C_{1} \cdot C_{3}}}{2 \cdot C_{1}}=166.869$

$q_{2}=\frac{-C_{2}-\sqrt{C_{2}^{2}-4 \cdot C_{1} \cdot C_{3}}}{2 \cdot C_{1}}=-240.314$

$c_{A}:=q_{1}=166.869$

$E I_{c r}:=\frac{E_{c} \cdot b \cdot c_{A}^{3}}{3}+E_{s} \cdot A_{s} \cdot\left(d-c_{A}\right)^{2}=7.536 \cdot 10^{13}$

$\varepsilon_{b i}:=\frac{M_{D L} \cdot\left(h-c_{A}\right)}{E I_{G Y}}=5.295 \cdot 10^{-4}$

Step 3: FRP system

$C_{E}:=0.95 \quad$ carbon and interior exposure

$f_{f t u}:=C_{E} \cdot f_{f}^{\prime}=617.5$

$\varepsilon_{j u}:=C_{E} \cdot \epsilon_{j u}^{\prime}=0,016$

$E_{f}:=\frac{f_{f u}}{\varepsilon_{f u t}}=3.824 \cdot 10^{4}$

Step 4: Design FRP reinforcment

$n:=1 \quad$ assume 1 layer for initial calculations

$\varepsilon_{f l}:=0.41 \cdot \sqrt{\frac{f_{c}^{\prime}}{\left(n \cdot E_{i} \cdot t_{f}\right)}}=0.014$ 


$$
\begin{aligned}
& \varepsilon_{f \in}:=\text { if } \varepsilon_{f a}<0.9 \cdot \varepsilon_{f u} \\
& \begin{array}{l}
\| \varepsilon_{f d} \\
\| 0.9 \cdot \varepsilon_{f a}
\end{array} \\
& \varepsilon_{f e}=0.014 \\
& x_{1}:=0.85 \\
& c_{b}=\frac{.003 \cdot h}{.003+\varepsilon_{f \varepsilon}+\varepsilon_{b i}}=106.208 \\
& \varepsilon_{s}=\frac{003}{c_{b}}\left(d-c_{b}\right)=0.012
\end{aligned}
$$

calculate the area required for $B S C$ depending if the steel yeilded:

$$
\begin{gathered}
A_{f_{z}}=\text { if } \varepsilon_{s}>\frac{f_{y}}{E_{s}} \\
\| \frac{\left(0.85 \cdot f_{c}^{\prime} \cdot \beta_{1} \cdot b \cdot c_{b}-A_{F} \cdot f_{y}\right)}{E_{f} \cdot \varepsilon_{f e}} \\
\quad \| \frac{\left(0.85 \cdot f_{c}^{\prime} \cdot \beta_{1} \cdot b \cdot c_{b}-A_{s} \cdot E_{s} \cdot \varepsilon_{s}\right)}{E_{f} \cdot \varepsilon_{f e}} \mid
\end{gathered}
$$

$A_{f b}=330.388$

determine the number of layers assuming full base of the beam:

$n=\left(\frac{A_{f b}}{\left(t_{\vec{f}} \cdot b\right)}\right)=0.974$

Step 5: configure FRP reinforcement

$$
n=11 \quad b_{f}:-381 \quad \quad \quad A_{f}=n \cdot b_{f} \cdot t_{f}=3.73 \cdot 10^{3}
$$


Step 6: calculate new moment capacity

$\varepsilon_{f d l}:=0.41 \cdot \sqrt{\frac{f^{\prime}}{\left(n \cdot E_{f} \cdot t_{f}\right)}}=0.004$

$\varepsilon_{f e}=$ if $\varepsilon_{f l l}<0.9 \cdot \varepsilon_{\gamma} \quad \varepsilon_{f e}=0.004$

$$
\begin{aligned}
& \| s_{y d} \\
& \text { else } \\
& \| 0.9 \cdot \sigma_{j u}
\end{aligned}
$$

$A_{f}<A_{f 0}=0 \quad$ false, therefore WSC design

assume steel yeilds:

$C_{1}=0.85 \cdot f_{c}^{\prime} \cdot \beta_{1} \cdot b=9.53 \cdot 10^{3}$

$C_{2}=E_{f} \cdot A_{f} \cdot\left(\varepsilon_{f e}+\varepsilon_{32}\right)-A_{s} \cdot f_{u}=-1.744 \cdot 10^{5}$

$C_{3}:=-h \cdot E_{f} \cdot A_{f} \cdot 003=-2.61 \cdot 10^{3}$

$$
q_{1}=\frac{-C_{2}+\sqrt{C_{2}^{2}-4 \cdot C_{1} \cdot C_{3}}}{2 \cdot C_{1}}=174.89
$$

$q_{2}=\frac{-C_{2}-\sqrt{C_{2}^{2}-4 \cdot C_{1} \cdot C_{3}}}{2 \cdot C_{1}}=-156.586$

$c:=q_{1}=174.89$

$\varepsilon_{s}:=\frac{.003}{e}(d-c)=0.006$

$e_{s}>.005=1 \quad$ tension controlled state

$M_{n}:=A_{s} \cdot f_{g} \cdot\left(d-\beta_{1} \cdot \frac{c}{2}\right)+85 * A_{f} \cdot E_{f} \cdot \varepsilon_{f \varepsilon} \cdot\left(h-\beta_{1} \cdot \frac{c}{2}\right)=6.729 \cdot 10^{8}$

$\phi=0.9$ because of tension controlled state

$\phi M_{n}=\phi \cdot M_{n}=6.056 \cdot 10^{8}$

$\phi M_{n}>M_{k}=1 \quad$ meets required loading capacity. 
Step 7: check serviceability requirements

$C_{1}:=E_{c} \cdot \frac{b}{2}=5.519 \cdot 10^{6}$

$C_{2}:=E_{s} \cdot A_{3}+E_{f} \cdot A_{f}=5.48 \cdot 10^{8}$

$C_{3}:=-E_{s} \cdot A_{s} \cdot d-E_{f} \cdot A_{f} \cdot h=-3.083 \cdot 10^{11}$ $q_{1}:=\frac{-C_{2}+\sqrt{C_{2}^{4}-4 \cdot C_{1} \cdot C_{3}}}{2 \cdot C_{1} \quad}=191.869$

$q_{2}:=\frac{-O_{2}-\sqrt{O_{2}{ }^{2}-4 \cdot C_{1} \cdot C_{3}}}{2 \cdot C_{1}}=-291.153$

$c_{B}:=q_{1}=191.869$

neutral axis of stage $B$ of serviceability requirements

concrete stress

$f_{c S t \cos t}=\frac{E_{c} \cdot\left(M_{D L} \cdot c_{A}\right)}{c_{A} \cdot b \cdot \frac{c^{3}}{3}+E_{s} \cdot A_{s} \cdot\left(d-c_{A}\right)^{2}}=5.777$

$f_{\text {stateB }}:=\frac{E_{c} \cdot\left(M_{L E} \cdot c_{B}\right)}{E_{c} \cdot b \cdot \frac{c_{B}^{3}}{3}+E_{*} \cdot A_{s} \cdot\left(d-c_{B}\right)^{2}+E_{f} \cdot A_{f} \cdot\left(h-c_{B}\right)^{2}}=11.182$

$f_{c}=f_{C S t a g e A}+f_{\operatorname{cotag} e B}=16.96$

$f_{s} \leq 0.45 \cdot f^{\prime}=1 \quad$ does not meet stress limits for concrete.

steel stress

$f_{s b c t}:=\frac{E_{s} \cdot\left(M_{D L} \cdot\left(d-c_{A}\right)\right)}{c_{A}^{3}+E_{c} \cdot A_{s} \cdot\left(d-c_{B}\right)^{2}}=90.612$

$f_{\text {squage } B}:=\frac{E_{s} \cdot\left(M_{L L} \cdot\left(d-c_{B}\right)\right)}{E_{c} \cdot b \cdot \frac{c_{B}^{3}}{3}+E_{s} \cdot A_{s} \cdot\left(d-c_{B}\right)^{2}+E_{f} \cdot A_{f} \cdot\left(h-c_{B}\right)^{2}}=142.472$

$f_{s}=f_{S S t a g e d}+f_{s \operatorname{sigg} B}=233.084 \quad f_{s} \leq 0.8 \cdot f_{3}=1$ meets stress limits for steel 
FRP stress

$f_{f}:=f_{s} \cdot\left(\frac{E_{f}}{E_{s}}\right) \cdot \frac{\left(n-c_{B}\right)}{\left(d-\epsilon_{B}\right)}-\varepsilon_{b v} \cdot E_{j}=32.366$

$f_{f} \leq 0.55 \cdot f_{f u}=1 \quad$ meets stress limits for glass FRP 


\section{Example E}

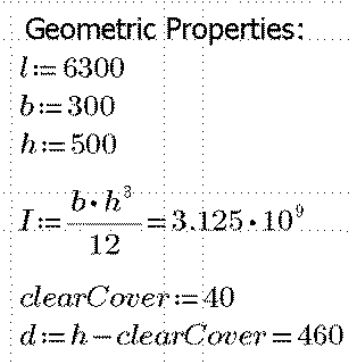

Steel Properties:

$A_{52}=141.7$

$s_{s}:=200$

$E_{s}:=200000$

$f_{y}: 275.79$

\section{Concrete properties:}

$f_{c}^{\prime}:=20,68$

$E_{c}=4700 \cdot \sqrt{f_{c}^{\prime}}=2.137 \cdot 10^{4}$

Loading Requirements:

$D L:=23 \quad V_{D L}=.5 \cdot D L \cdot l=7.245 \cdot 10^{4}$

$L L:=12 \cdot 1.5=18 \quad V_{L E}=5 \cdot L L \cdot l=5.67 \cdot 10^{4}$

$w_{2}:=1.2 \cdot D L+1.6 \cdot L L=56.4$

$V_{u}:=\frac{1}{2} \cdot w_{* h} \cdot l=1.777 \cdot 10^{5}$

FRP Properties:

$f_{f}^{\prime}:=3700$

$\varepsilon_{f t u}^{\prime}:=.016$

$t_{1}=0.200$

carbon.

interior exposure$$
0^{4}
$$

Step 1: Original Shear capacity and maximum allowable strengthening

$V_{c}:=\frac{1}{6} \cdot b \cdot d \cdot \sqrt{f_{c}^{\prime}}=1.046 \cdot 10^{5}$

$V_{s}:=\frac{f_{y} \cdot A_{s i v} \cdot d}{s_{s}}=8.988 \cdot 10^{\frac{1}{E}}$

$\phi:=0.75$

$\phi V_{s}:=\phi \cdot\left(V_{c}+V_{s}\right)=1.459 \cdot 10^{5}$

check minimum loading capacity

$S_{u n s t r}:=1.1 \cdot V_{D S}+.75 \cdot V_{L L}=1.222 \cdot 10^{5}$

$\phi V_{\text {m }} \geq S_{\text {whst }}=1$ true.

check maximum allowable strengthening:

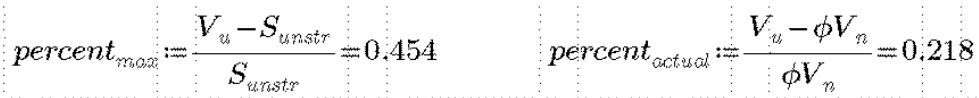

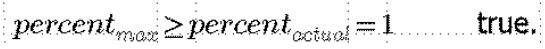

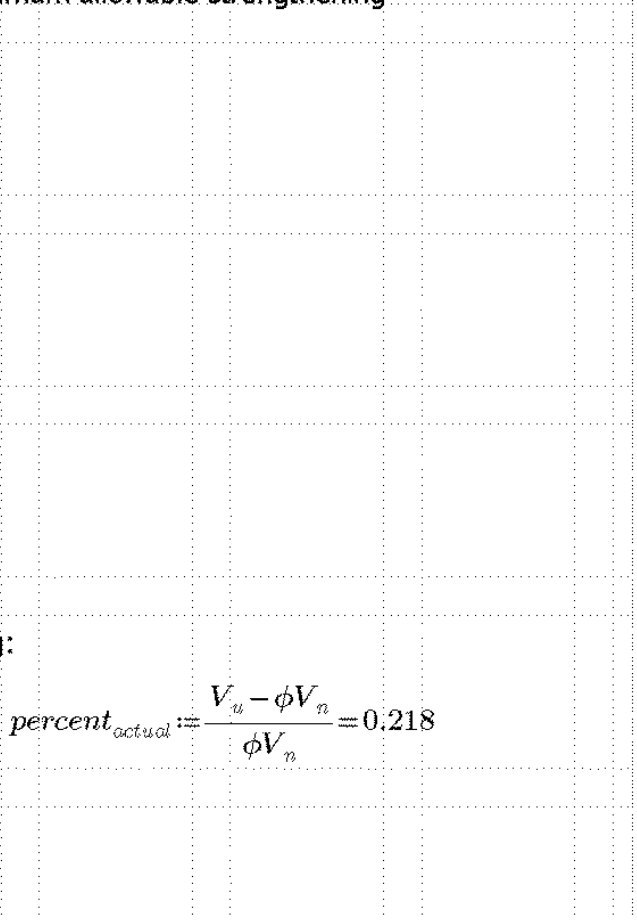


Step 2: FRP system

$C_{Z 1}:=0.95 \quad$ carbon and interior exposure

$f_{f f}:=C_{E} \cdot f_{f}^{\prime}=3.515 * 10^{3}$

$\varepsilon_{f u}:=C_{E} \cdot e_{f u}^{t}=0.015$

$E_{f}:=\frac{f_{f \omega}}{\varepsilon_{f u t}}=2.313 \cdot 10^{5}$

Step 3: Determine the number of layers needed and the geomoetry of the reinforcement:

3-sided wrapping scheme

$a_{p v}:=300$

$\alpha_{f}:=\frac{\pi}{2}=1.571 \ldots \psi_{f}:=0.85$

assume initial complete continuous wrapping

$\varepsilon_{f e}:=$ if $0.75 \cdot \varepsilon_{f w}>004$

$\| 004$

else

$\| 0.75 \cdot \varepsilon_{f(x)}$

$\varepsilon_{f \mathrm{ft}}=0,004$

$V_{R f}:=\frac{1}{\psi_{f}} \cdot\left(\frac{V_{u}}{\phi}-V_{c}-V_{s}\right)=4.989 \cdot 10^{4}$

$n:=\frac{V_{R j}}{\left(2 \cdot \varepsilon_{f} \cdot E_{f} \cdot t_{f} \cdot d_{p_{2}} \cdot \sin \left(\alpha_{f}\right) \cdot\left(\sin \left(\alpha_{f}\right)+\cos \left(\alpha_{f}\right)\right)\right)}=0.449$

$n:=\operatorname{ceil}(n)=1$

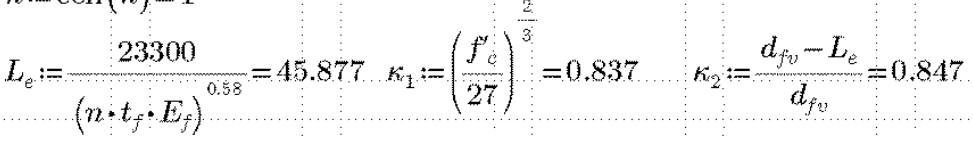

$\kappa_{\vartheta}:=\frac{\kappa_{1} \cdot \kappa_{2} \cdot L_{e}}{11900 \cdot \varepsilon_{f u}}=0.18$

$\kappa_{v}<0.75=1 \quad$ true 


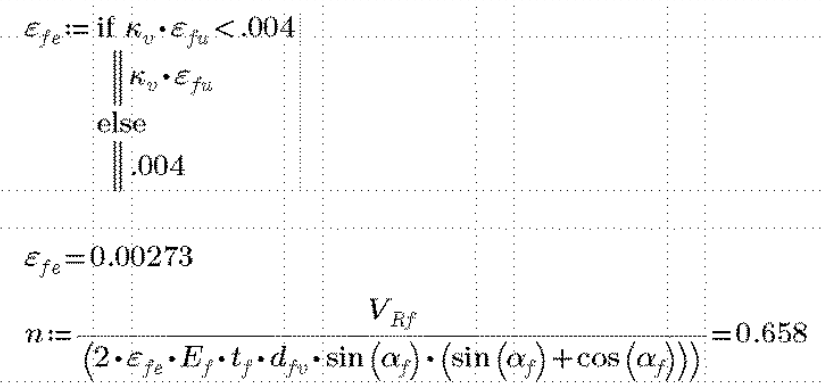

$n:=\operatorname{ceil}(n)=1$

Step 4: configure FRP reinforcement

$n:=1 \quad$ in three-sided wrapping

$d_{f v}=300$

$\alpha_{f}=\frac{\pi}{2}=1.571$

Step 5: determine the shear capacity

$V_{f}:=2 \cdot n \cdot t_{f} \cdot E_{f} \cdot \varepsilon_{j e} \cdot d_{f v}=7.586 \cdot 10^{6}$

$V_{f} \geq V_{R f}=1 \quad$ meets required loading capacity

Step 6: Check maximum allowable shear reinforcement

$$
\begin{aligned}
& V_{f}+V_{s} \leq 0.66 \cdot \sqrt{f_{c}^{\prime}} \cdot b \cdot d=1 \quad \text { meets allowable } \\
& \text { reinforcement requirements }
\end{aligned}
$$

$V_{f}+V_{s}=1.657 \cdot 10^{5}$

$0.66 \cdot \sqrt{f_{0}^{\prime}} \cdot b \cdot d=4.142 \cdot 10^{5}$

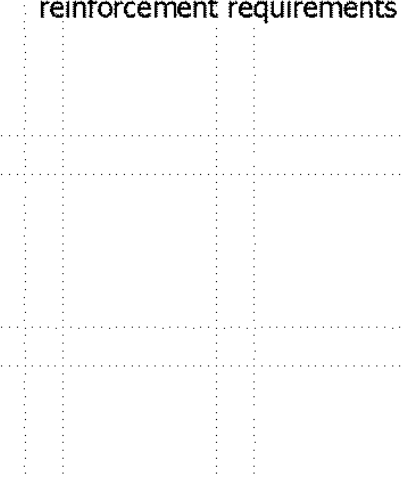




\section{Example F}

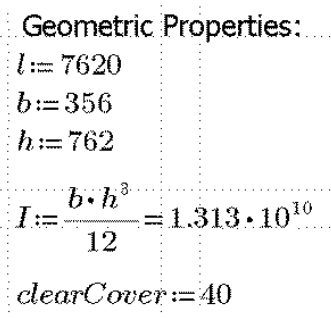

Steel Properties:

$A_{3 v}:=258$

$s_{s}:=355.6$

$E_{s}:=200000$

$f_{3}:=414$

\section{Concrete properties:}

$f_{x}^{\prime}:=27.6$

$E_{c}=4700 \cdot \sqrt{f_{c}^{\prime}}=2.469 \cdot 10^{4}$

Loading Requirements:

$D L:=6.392 \quad V_{D L}=.5 \cdot D L \cdot l=2.435 \cdot 10^{4}$

$L L=100$

$V_{L L}=.5 \cdot L L \cdot l=3.81 \cdot 10^{5}$

$w_{1 k}:=1.2 \cdot D L+1.6 \cdot L L=16767$

$V_{u t}:=\frac{1}{2} \cdot w_{t h} \cdot l=6.388 \cdot 10^{5}$

FRP Properties:

$f_{f:=3481.9}^{\prime}$

$\varepsilon_{f \text { tut }}^{\prime}:=.0153$

$t_{1}=0.254$

carbon.

interior exposure

$$
\cdot 10^{4}
$$

Step 1: Original Shear capacity and maximum allowable strengthening

$V_{c}:=\frac{1}{6} \cdot b \cdot d \cdot \sqrt{f_{c}^{\prime}}=2.251 \cdot 10^{5}$

$V_{s}:=\frac{f_{y} \cdot A_{s v} \cdot d}{s_{s}}=2.169 \cdot 10^{5}$

$\phi:=0.75$

$\phi V_{n_{1}}:=\phi \cdot\left(V_{c}+V_{s}\right)=3.314 \cdot 10^{5}$

check minimum loading capacity

$S_{u n s t r}:=1.1 \cdot V_{D S}+.75 \cdot V_{L L}=3.125 \cdot 10^{5}$

$\phi V_{n^{n}} \geq S_{\text {w }}=1$ true

check maximum allowable strengthening:

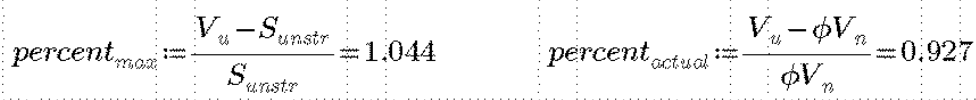

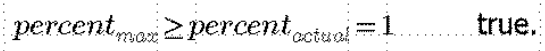


Step 2: FRP system

$C_{Z 1}:=0.95 \quad$ carbon and interior exposure

$f_{f f r}:=C_{E} \cdot f_{f}^{\prime}=3.308 \cdot 10^{3}$

$c_{f u}:=C_{E} \cdot \varepsilon_{f u}^{z}=0.015$

$E_{f}:=\frac{f_{f \omega}}{\varepsilon_{f u t}}=2.276 \cdot 10^{5}$

Step 3: Determine the number of layers needed and the geomoetry of the reinforcement: complete wrapping scheme $a_{j v}:=h$

$\alpha_{f}:=\frac{\pi}{2}=1.571 \ldots \psi_{f}:=0.95$

$\varepsilon_{y e}:=$ if $0.75 \cdot \varepsilon_{f u t}>004$

$\| 004$

else

$\| 0.75 \cdot \varepsilon_{j t}$

$\varepsilon_{f e}=0,004$

$V_{R f}:=\frac{1}{\psi_{t}} \cdot\left(\frac{V_{u}}{\phi}-V_{o}-V_{s}\right)=4.314 \cdot 10^{b}$

$n:=\frac{V_{R f}}{\left(2 \cdot \varepsilon_{f e} \cdot E_{f} \cdot t_{f} \cdot d_{f_{p}} \cdot \sin \left(\alpha_{f}\right) \cdot\left(\sin \left(\alpha_{f}\right)+\cos \left(\alpha_{f}\right)\right)\right)}=1.224$

$n:=\operatorname{ceil}(n)=2$

Step 4: configure FRP reinforcement

$n:=2 \quad$ complete wrapping

$d_{p_{v}}=h$

$\alpha_{y}:=\frac{\operatorname{mor}}{2}=1.571$

$w_{f}:=305$ 
$s_{\text {HMas }}:=\frac{2 \cdot n \cdot t_{j} \cdot w_{j} \cdot d_{j p} \cdot E_{j} \cdot c_{j e}=498243}{V_{R f}}$

Step 5: determine the shear capacity

$V_{f}:=\frac{2 \cdot n \cdot t_{f} \cdot L_{f} \cdot \varepsilon_{f e} \cdot d_{f v} \cdot w_{f} \cdot \sin \left(\alpha_{f}\right) \cdot\left(\sin \left(\alpha_{f}\right)+\cos \left(\alpha_{f}\right)\right)}{s_{f}}=5.374 \cdot 10^{5}$

$V_{f} \geq V_{R f}=1 \quad$ meets required loading capacity

Step 6: Check maximum allowable shear reinforcement

$V_{f}+V_{s} \leq 0.66 \cdot \sqrt{f_{c}^{\prime}} \cdot b \cdot d=1 \quad$ meets allowable reinforcement requirements

$V_{f}+V_{s}=7.542 \cdot 10^{b}$

$0.66 \cdot \sqrt{f_{c}^{\prime}} \cdot b \cdot d=8.912 \cdot 10^{5}$

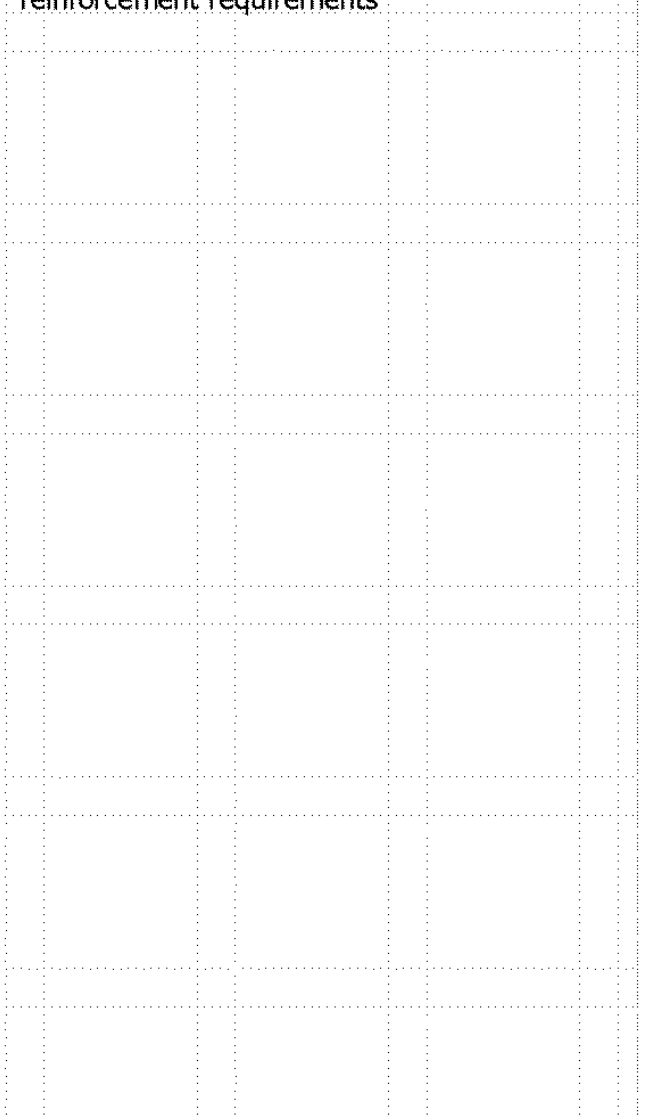




\section{Example G}

Geometric Properties:
$l:=4000$
$b:=381$
$h:=635$
$I:=\frac{b \cdot h^{3}}{12}=8.13 \cdot 10^{5}$
clearCover $:=76.2$

Steel Properties:

$A_{52}:=113.14$

$s_{s}:=200$

$E_{s}:=200000$

$f_{y}:=275.79$

Concrete properties:

$f_{c}^{\prime}:=20,68$

$d:=h-$ clearCover $=558.8$
$E_{c}:=4700 \cdot \sqrt{f_{c}}=2.137 \cdot 10^{4}$

FRP Properties:

$f_{f:-3481.9}^{\prime}$

$\varepsilon_{f+t:}^{\prime}:=.0153$

$t_{t}=0.254$

carbon.

interior exposure

Loading Requirements:

$D L:=21.997+23.996 \quad V_{D L}=.5 \cdot D L \cdot l=9.199 \cdot 10^{4}$

$L L=25.998 \cdot 2.5 \quad V_{L I}=.5 \cdot L L \cdot l=1.3 \cdot 10^{5}$

$w_{u}:=1.2 \cdot D L+1.6 \cdot L L=159184$

$V_{u}:=\frac{1}{2} \cdot w_{t h} \cdot l=3.184 \cdot 10^{5}$

Step 1: Original Shear capacity and maximum allowable strengthening

$V_{c}:=\frac{1}{6} \cdot b \cdot d \cdot \sqrt{f_{c}^{\prime}}=1.614 \cdot 10^{5}$

$V_{s}:=\frac{f_{y} \cdot A_{s i} \cdot d}{s_{s}}=8.718 \cdot 10^{\frac{1}{6}}$

$\phi:=0.75$

$\phi V_{n}:=\phi \cdot\left(V_{c}+V_{s}\right)=1.864 \cdot 10^{5}$

check minimum loading capacity

$S_{\text {unstr }}:=1.1 \cdot V_{D S}+.75 \cdot V_{L L}=1.987 \cdot 10^{5}$

$\phi V_{\text {m }} \geq S_{\text {whst }}=0$ true.

check maximum allowable strengthening:

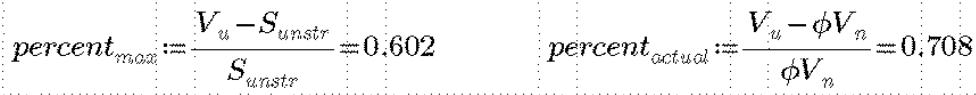

percent max $_{\text {max }} \geq$ percent $_{\text {act }}=0 \quad$ true

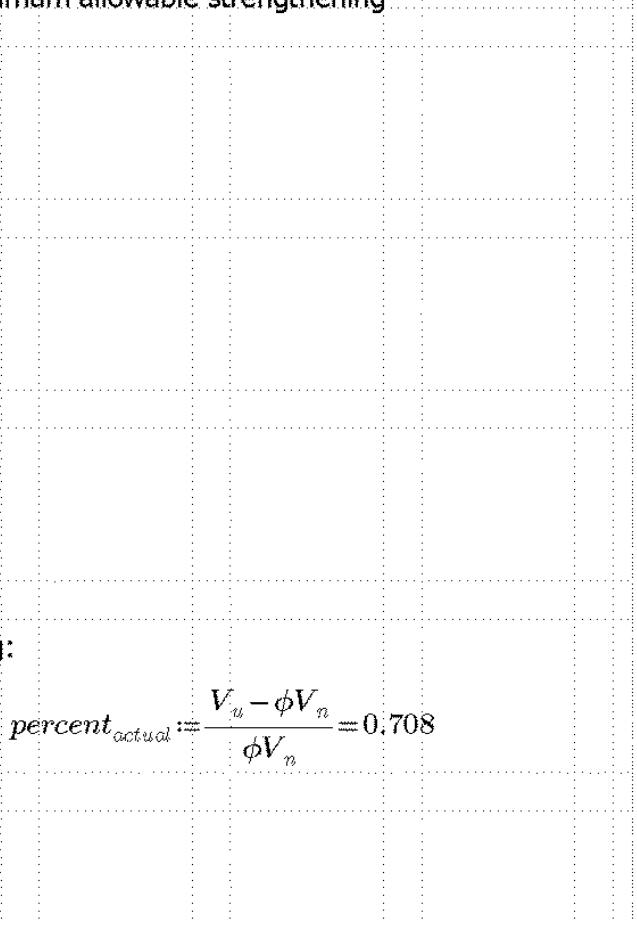


Step 2: FRP system

$C_{Z 1}:=0.95 \quad$ carbon and interior exposure

$f_{f f r}:=C_{E} \cdot f_{f}^{\prime}=3.308 \cdot 10^{3}$

$c_{f u}:=C_{E} \cdot \varepsilon_{f u}^{z}=0.015$

$E_{f}:=\frac{f_{f \omega}}{\varepsilon_{f u t}}=2.276 \cdot 10^{5}$

Step 3: Determine the number of layers needed and the geomoetry of the reinforcement

2-sided wrapping scheme

$a_{f v}:=406.4$

$\alpha_{f}:=\frac{\pi}{2}=1.571 \ldots \psi_{f}:=0.85$

assume initial complete continuous wrapping

$\varepsilon_{f e}:=$ if $0.75 \cdot \varepsilon_{f w}>0004$

$\| 004$

else

$\| 0.75 \cdot \varepsilon_{f(x)}$

$\varepsilon_{f \mathrm{ft}}=0,004$

$V_{R f}:=\frac{1}{\psi_{f}} \cdot\left(\frac{V_{u}}{\phi}-V_{c}-V_{s}\right)=2.07 \cdot 10^{5}$

$n:=\frac{V_{R f}}{\left(2 \cdot \varepsilon_{p e} \cdot \dot{E}_{f} \cdot t_{f} \cdot d_{p_{2}} \cdot \sin \left(\alpha_{f}\right) \cdot\left(\sin \left(\alpha_{f}\right)+\cos \left(\alpha_{f}\right)\right)\right)}=1.101$

$n:=\operatorname{ceil}(n)=2$

$L_{e}:=\frac{23300}{\left(n+t_{f}, E_{f}\right)^{0.58}}=26.966 \kappa_{1}:=\left(\frac{f^{4}}{27}\right)^{\frac{2}{3}}=0.837 \quad \kappa_{2}:=\frac{d_{f_{v}}-2 \cdot L_{e}}{d_{f v}}=0.867$

$\kappa_{v}:=\frac{\kappa_{1} \cdot \kappa_{2} \cdot L_{2}}{11900 \cdot \varepsilon_{f u}}=0.113$

$n_{v}<0.75=1$ true 


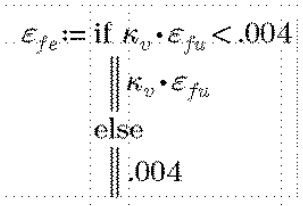

Step 4: configure FRP reinforcement

$n:=3 \quad 2 \quad 2$-sided wrapping

$d_{f, \gamma}=406.4$

$\alpha_{f}=\frac{\pi}{2}=1.571$

$w_{f}:=305$

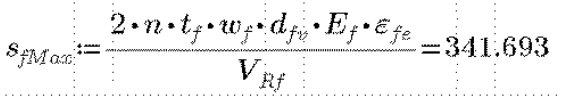

$s_{f}:=500$

Step 5: determine the shear capacity

$V_{f}:=\frac{2 \cdot m \cdot t_{f} \cdot E_{f} \cdot \varepsilon_{f e} \cdot d_{f} \cdot w_{f} \cdot \sin \left(\alpha_{f}\right) \cdot\left(\sin \left(\alpha_{f}\right)+\cos \left(\alpha_{f}\right)\right)}{s_{f}}=1.415 \cdot 10^{5}$

$V_{f} \geq V_{R f}=0 \quad$ meets required loading capacity

Step 6: Check maximum allowable shear reinforcement

$$
V_{f}+V_{s} \leq 0.66 \cdot \sqrt{f^{\prime}} \cdot b \cdot d=1 \quad \text { meets allowable }
$$
reinforcement requirements

$V_{f}+V_{s}=2.286 \cdot 10^{5}$

$0.66 \cdot \sqrt{f_{c}^{\prime}} \cdot b \cdot d=6.39 \cdot 10^{5}$ 


\section{Example $\mathbf{H}$}

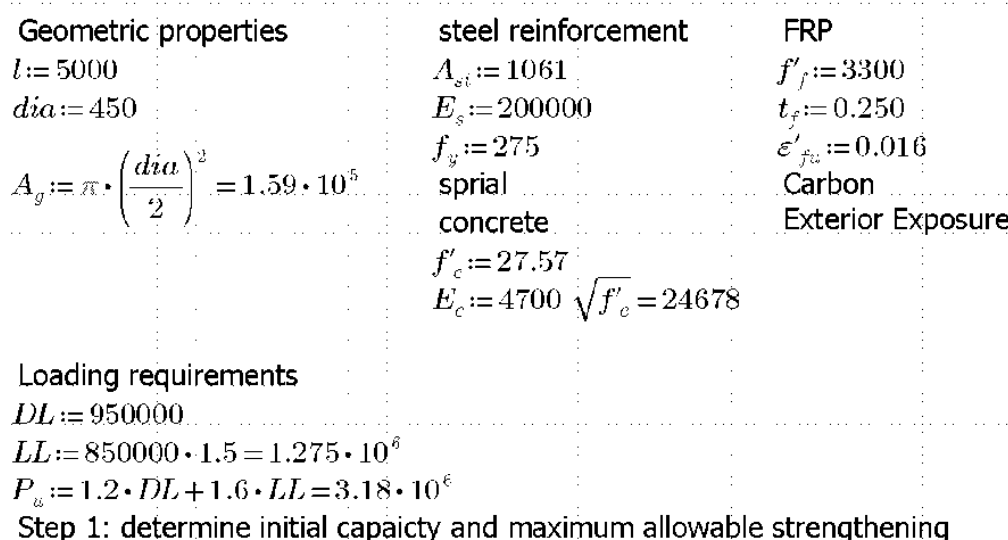

$\phi:=0.75 \quad \phi_{\text {гve }}:=0.85$

$\phi P_{n}:=\phi \cdot \phi_{s s c} \cdot\left(0.85 \cdot f_{i}^{\prime} \cdot\left(A_{2}-A_{s t}\right)+f_{i j}^{\prime} \cdot A_{s i}\right)=2.546 \cdot 10^{6}$

check minimum loading capacity:

$$
\begin{aligned}
& S_{\text {wast }}:=1.1 \cdot D L+.75 \cdot L L=2.001 \cdot 10^{6} \\
& \phi P_{n} \geq S_{\text {insist }} \rightarrow 1 \quad \text { true. }
\end{aligned}
$$

check maximum allowable strengthening:

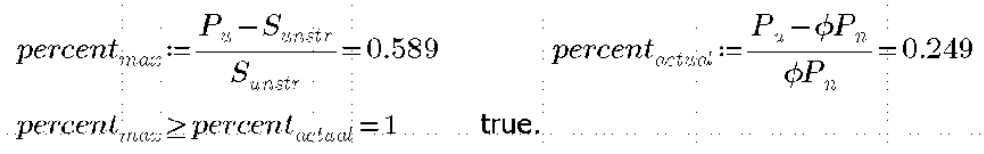

Step 2: FRP system

$C_{E}:=0.85 \ldots$ carbon and exterior exposure

$f_{f_{b}}:=C_{E} \cdot f_{i}^{\prime \prime}=2.805 \cdot 10^{3}$

$\varepsilon_{f \alpha x}:=C_{E} \cdot \varepsilon_{f u x}^{\prime}=0.014$

$E_{f}^{\prime}:=\frac{f_{f u}}{\varepsilon_{i j h}}=2.063 \cdot 10^{f}$ 
Step $3:$ determine the number of layers needed

$$
\begin{aligned}
& \kappa_{\theta}:=1 \quad \text { circular cross-section }
\end{aligned}
$$

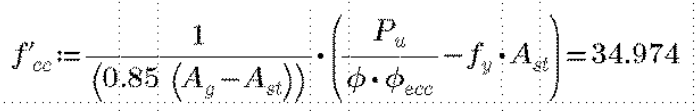

$$
\begin{aligned}
& \psi_{f}:=0.95 \\
& f_{l}:=\frac{f_{c}^{\prime}-f_{c}^{t}}{\psi_{j} \cdot 3.3 \cdot \kappa_{a}}=2.362 \\
& n:=\frac{\operatorname{dia} f_{l}}{2 \cdot t_{f} \cdot E_{f} \cdot \varepsilon_{f^{2}} \cdot 0.55}=1.378 \\
& n:=\operatorname{coil}(n)=2 \\
& \text { Step 4: configure FRP reinforcement } \\
& n:=2
\end{aligned}
$$

Step 5: determine axial capacity of strengthened column

$f_{l}=\frac{2 \cdot E_{f} \cdot n+t_{f} \cdot \varepsilon_{f u}+55}{\text { dia }}=3,428$

$$
f_{c c}^{\prime}:=f_{c}^{\prime}+3.3 \cdot \psi_{f} \cdot \kappa_{a} \cdot f_{l}=38.318
$$

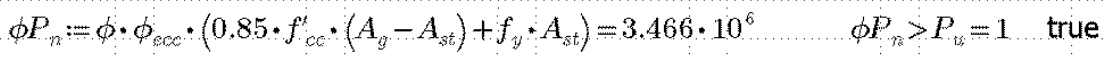$$
\frac{f_{l}}{f_{c}^{\prime}}=0.124 \quad \frac{f_{l}}{f^{\prime}}>0.08=1 \quad \text { true }
$$$$
\kappa_{3}:=1 \quad \text { circular cross-section }
$$$$
\varepsilon_{c e x}:=.002 \cdot\left(1.5+12 \cdot \kappa_{0} \cdot \frac{f_{l}}{f_{c}^{\prime}} \cdot\left(\frac{w_{f \alpha} \cdot .55}{.002 \vdots}\right)^{45}\right)=0.0084
$$$$
\varepsilon_{c c u s}<.01=1 \quad \text { true }
$$$$
P_{s}=D L+L L=2.225 \cdot 10^{6}
$$$$
f_{c}:=\frac{E_{c} \cdot P_{s}}{E_{c} \cdot\left(A_{p}-A_{s t}\right)+E_{s} \cdot A_{s t}}=13.357
$$

$f_{c} \leq 0.65 \cdot f_{c}^{\prime}=1 \quad$ true

$$
\begin{gathered}
f_{s}:=\frac{E_{s} \cdot P_{s}}{E_{c} \cdot\left(A_{t}-A_{s t}\right)+E_{s} \cdot A_{s t}}=108.248 \\
f_{s} \leq .6 \cdot f_{y}=1 \text { true }
\end{gathered}
$$




\section{Example I}

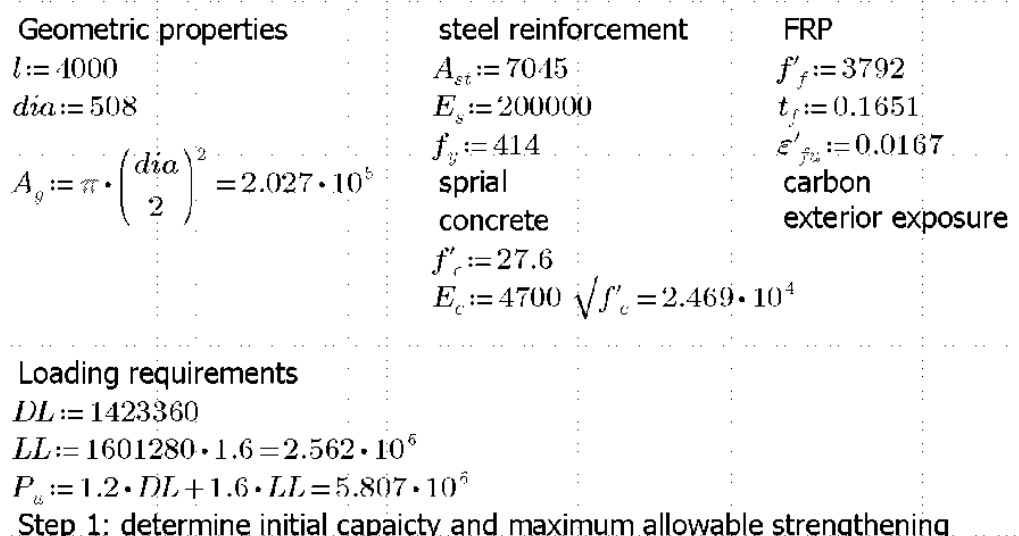

$\phi:=0.75 \quad \phi_{c c c}:=0.85$

$\phi P_{n}:=\phi \cdot \phi_{s c c} \cdot\left(0.85 \cdot f_{c}^{t} \cdot\left(A_{g}-A_{s t}\right)+f_{y^{\prime}} \cdot A_{s t}\right)=4.785 \cdot 10^{6}$

check minimum loading capacity:

$$
\begin{aligned}
& S_{\text {u. } r s^{5}, t}:=1.1 \cdot D L+.75 \cdot L L=3.487 \cdot 10^{6} \\
& \phi P_{13} \geq S_{3, n \in t r} \rightarrow 1 \quad \text { true. }
\end{aligned}
$$

check maximum allowable strengthening:

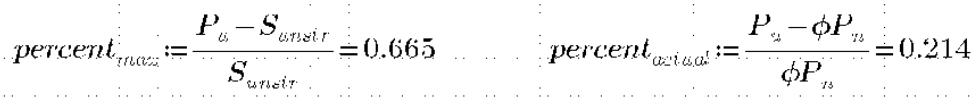

percent $_{\text {muive }} \geq$ percent $_{\text {uctulul }}=1$ true.

Step 2: FRP system

$C_{E}=0.85 \ldots$ carbon and exterior exposure

$f_{i \omega_{i}}:=C_{E} \cdot f_{i}^{\prime}=3.223 \cdot 10^{3}$

$\varepsilon_{f \alpha}:=C_{E} \cdot \varepsilon_{f u}^{\prime}=0.014$

$E_{f}:=\frac{f_{f u}}{\varepsilon_{f \cdot i}}=2.271 \cdot 10^{5}$ 
Step 3: determine the number of layers needed

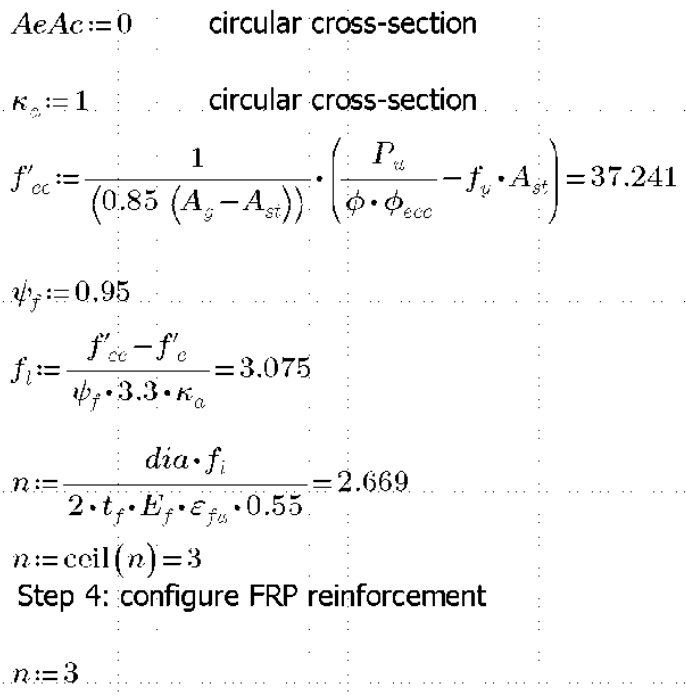




\section{Example J}

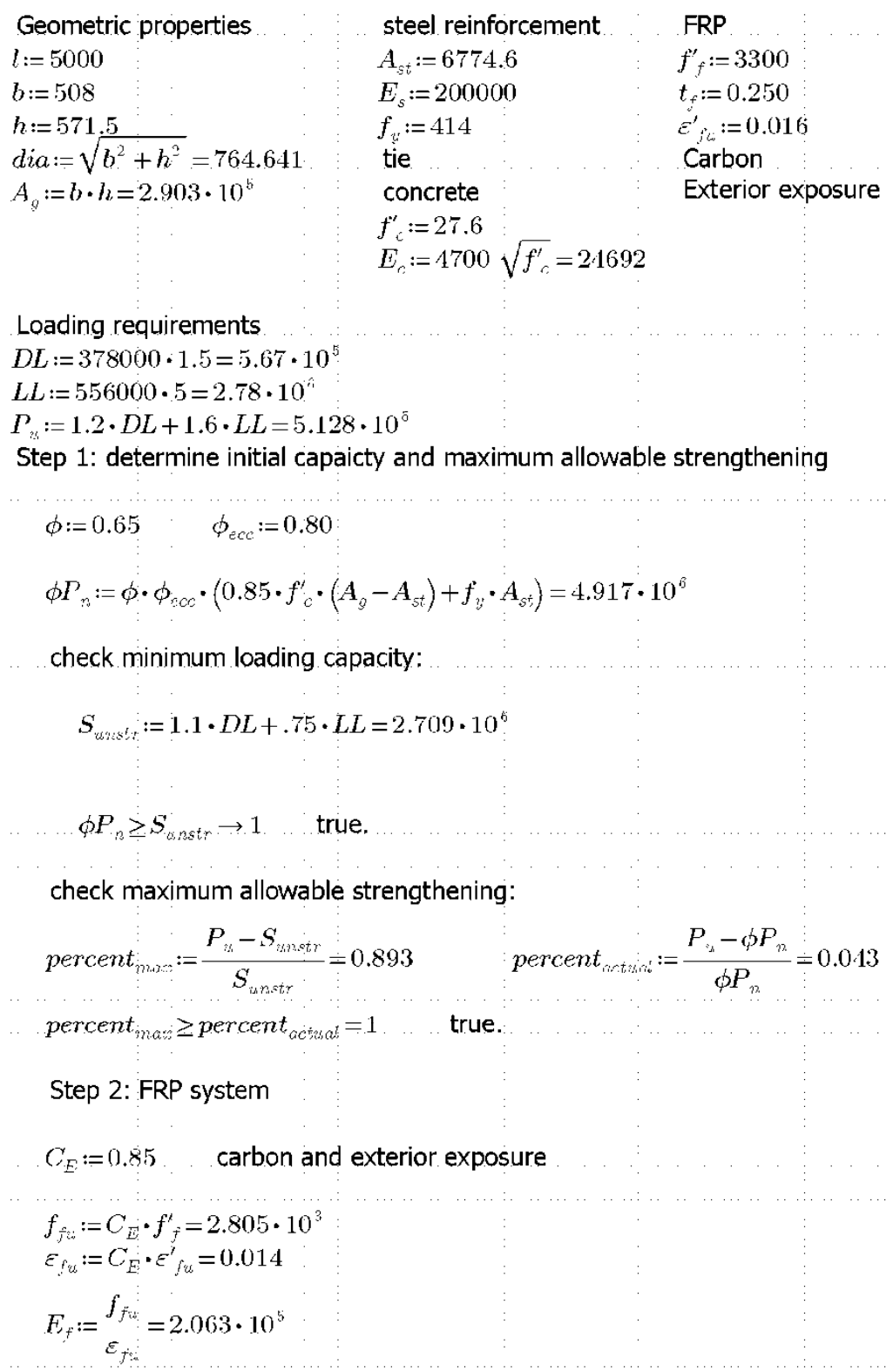


Step 3: determine the number of layers needed

$$
\begin{aligned}
& A e A c:=\frac{1-\frac{\left(\frac{b}{h} \cdot(h-2 \cdot 25)^{2}+\frac{h}{b} \cdot(b-2 \cdot 25)^{2}\right)}{3 \cdot A_{g}}-\frac{A_{s i}}{A_{g}}}{\vdots}=0.319 \\
& \kappa_{0}:=A e A c \cdot\left(\frac{b^{\prime}}{h^{2}}\right)=0.252 \quad A^{\text {non-circular cross-section }} \\
& f_{c c}^{\prime}:=\frac{1}{\left(0.85\left(\Lambda_{i}-\Lambda_{c i}\right)\right)} \cdot\left(\frac{P_{w}}{\phi \cdot \phi_{c c c}}-f_{y} \cdot \Lambda_{s}\right)=29.283 \\
& \psi_{\bar{T}}:=0.95 \\
& f_{l}:=\frac{f_{c c}^{\prime}-f_{c}^{\prime}}{\psi_{f} \cdot 3.3 \cdot \kappa_{c}}=2.132 \\
& n:=\frac{d i a \cdot f_{i}}{2 \cdot t_{f} \cdot E_{j} \cdot c_{j e} \cdot 0.55}=2.113 \\
& n:=\operatorname{ceil}(n)=3 \\
& \text { Step 4: configure FRP reinforcement } \\
& n:=3
\end{aligned}
$$

Step 5: determine axial capacity of strengthened column

$$
f_{i}:=\frac{2 \cdot E_{f} \cdot n \cdot t_{j} \cdot \varepsilon_{f i} \cdot .55}{\text { dia }}=3.026
$$$$
f_{c i}^{\prime}:=f_{r}^{\prime}+3.3 \cdot \psi_{f} \cdot \kappa_{i j} \cdot f_{l}=29.989
$$$$
\phi P_{n}:=\phi \cdot \phi_{n c c} \cdot\left(0.85 \cdot f^{\prime} \cdot\left(A_{g}-A_{s^{5}}\right)+f_{y} \cdot A_{s t}\right)=5.217 \cdot 10^{6} \ldots \quad \phi P_{n}>P_{i w}=1 \text { true }
$$$$
\frac{f_{t}^{\prime}}{f_{c}^{\prime}}=0.11 \quad \frac{f_{l}}{f_{c}^{\prime}}>0.08=1 \quad \text { true }
$$$$
\kappa_{b}:=\operatorname{AeA}: \cdot\left(\frac{h}{b}\right)^{0.5}=0.338 \text { non-circular cross-section }
$$$$
\varepsilon_{\text {rcid }}:=.002 \cdot\left(1.5+12 \cdot \kappa_{b} \cdot \frac{f_{i}}{f_{c}^{\prime}} \cdot\left(\frac{\varepsilon_{f i} \cdot .55}{.002}\right)=0.0046\right.
$$$$
\varepsilon_{\text {oni }}<.01=1 \quad \text { true } \quad P_{s}:=D L+L L=3.347 \cdot 10^{6} \text {. }
$$

$f_{c}^{\prime}:=\frac{E_{c} \cdot P_{s}}{E_{c} \cdot\left(A_{g}-A_{s^{t}}\right)+E_{s} \cdot A_{s i}}=9.89$

$f_{c} \leq 0.65 \cdot f_{c}^{\prime}=1 \quad$ true

$$
f_{s}:=\frac{E_{s} \cdot P_{s}}{E_{c} \cdot\left(A_{g}-A_{s t}\right)+E_{s:} \cdot A_{s t}}=80.108
$$$$
\int_{s} \leq . \dot{b} \cdot \int_{y j}=1 \quad \text { true }
$$ 


\section{Example K}

Step 3: determine the number of layers needed

$$
\begin{aligned}
& A e A c:=\frac{1-\frac{\left(\frac{b}{h} \cdot(h-2 \cdot 25)^{2}+\frac{h}{b} \cdot(b-2 \cdot 25)^{2}\right)}{3 \cdot A_{s}}-\frac{A_{s i}}{A_{g}}}{1-\frac{A_{s i}}{A_{s i}}}=0.319 \\
& \kappa_{0}:=A e A c \cdot\left(\frac{b^{\prime}}{h^{2}}\right)=0.252: \quad \begin{array}{c}
A_{s} \\
\text { non-circular cross-section }
\end{array} \\
& f_{c c}^{\prime}:=\frac{1}{\left(0.85\left(\Lambda_{c}-\Lambda_{s i}\right)\right)} \cdot\left(\frac{P_{u}}{\phi \cdot \phi_{c c c}}-f_{y} \cdot \Lambda_{s t}\right)=29.283 . \\
& \psi_{T}:=0.95 \\
& f_{l}:=\frac{f_{c c}^{\prime}-f_{c}^{\prime}}{\psi_{f} \cdot 3.3 \cdot \kappa_{c}}=2.132 \\
& n:=\frac{d i a \cdot f_{i}}{2 \cdot t_{j} \cdot E_{j} \cdot c_{j c} \cdot 0.55}=2.113 \\
& n:=\operatorname{ceil}(n)=3
\end{aligned}
$$

Step 5: determine axial capacity of strengthened column

$$
f_{i}:=\frac{2 \cdot E_{f} \cdot \eta \cdot t_{j} \cdot \varepsilon_{f i} \cdot .55}{d i a}=3.026
$$$$
f_{m}^{\prime}:=f_{r}^{\prime}+3.3 \cdot \psi_{f} \cdot \kappa_{i} \cdot f_{l}=29.989
$$$$
\phi P_{n}:=\phi \cdot \phi_{n c e} \cdot\left(0.85 \cdot f_{c e}^{\prime} \cdot\left(A_{g}-A_{s i}\right)+f_{y} \cdot A_{s t}\right)=5.217 \cdot 10^{n} \ldots \phi P_{n}>P_{u b}=1 \text { true }
$$$$
\frac{f_{t}^{\prime}}{f_{c}^{\prime}}=0.11 \quad \frac{f_{l}}{f^{\prime}}>0.08=1 \quad \text { true }
$$$$
\kappa_{b}:=\operatorname{AeAc}_{c} \cdot\left(\frac{h}{b}\right)^{0.5}=0.338 \text { non-circular cross-section }
$$$$
\varepsilon_{\text {ccit }}=.002 \cdot\left(1.5+12 \cdot \kappa_{b} \cdot \frac{f_{i}}{f_{c}^{\prime}} \cdot\left(\frac{\varepsilon_{f i t} \cdot .55}{.002}\right)=0.0046\right.
$$$$
\varepsilon_{o w}<.01=1 \quad \text { true } \quad P_{s}:=D L+L L=3.347 \cdot 10^{6} \text {. }
$$

$$
\begin{array}{ll}
f_{c}^{\prime}:=\frac{E_{c} \cdot P_{s}}{E_{c} \cdot\left(A_{g}-A_{s t}\right)+E_{s} \cdot A_{s i}}=9.89 & f_{s}:=\frac{E_{s} \cdot P_{s}}{E_{c} \cdot\left(A_{g}-A_{s t}\right)+E_{s} \cdot A_{s t}}=80.108 \\
f_{c} \leq 0.65 \cdot f_{c}^{\prime}=1 \quad \text { true } & f_{s} \leq .6 \cdot f_{g j}=1 \quad \text { true }
\end{array}
$$




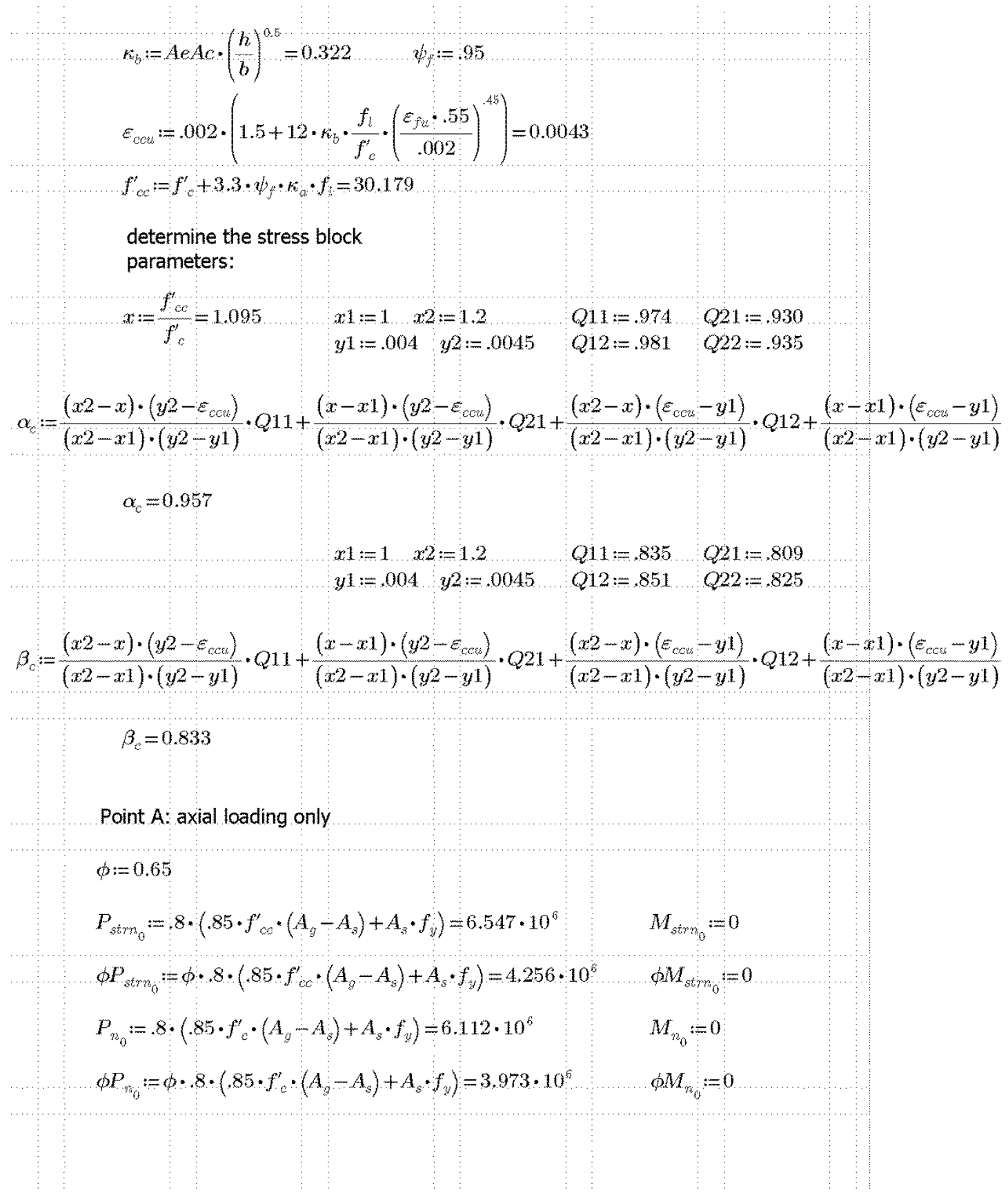


Point D: axial loading with minimum eccentricity

$$
\begin{aligned}
& P_{s t m m_{1}}:=P_{s t m_{0}}=6.547 \cdot 10^{6} \quad \quad M_{3 t m m_{1}}:=P_{s t m m_{1}} \cdot 1 \cdot h=3.274 \cdot 10^{8}
\end{aligned}
$$

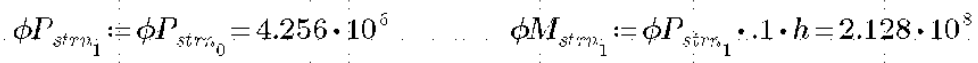

$$
\begin{aligned}
& P_{r_{1}}:=P_{s_{0}}=6.112 \cdot 10^{6} \quad \quad M_{r_{1}}:=P_{r_{1}} \cdot 1 \cdot h=3.056 \cdot 10^{8}: \\
& \phi P_{\eta_{1}}:=\phi P_{r_{b_{1}}}=3.973 \cdot 10^{5}: \quad \phi M_{\eta_{1}}:=\phi P_{r_{b_{1}}}: .1 \cdot h=1.986 \cdot 10^{*} \\
& c:=\frac{.003}{(.003+0)} \cdot d=460 \\
& \varepsilon_{\delta_{1}}:=\frac{.003}{c} \cdot\left(D_{s t_{0,1}}-c\right)=-0.0027 \quad \varepsilon_{\varepsilon_{1}}:=\frac{.003}{c} \cdot\left(D_{s t_{0,1}}-c\right)=-0.0018 \\
& \varepsilon_{s_{2}}:={ }_{c}^{.003} \cdot\left(D_{\varepsilon t_{0,2}}-c\right)=-0.0009 \quad \varepsilon_{s_{3}}:=0
\end{aligned}
$$

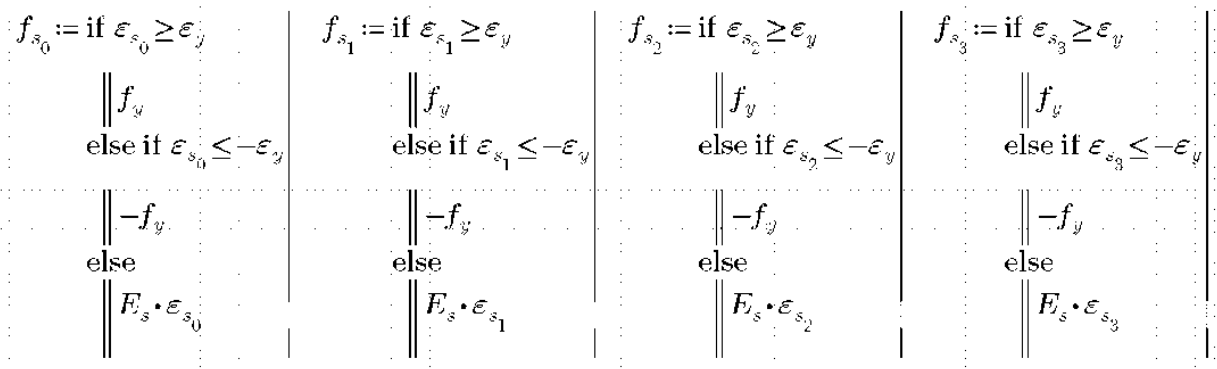

$$
\begin{aligned}
& f_{30}=-414 \\
& f_{\bar{s}_{1}}=-358.696 \\
& f_{s_{2}}=-189.13 \\
& f_{s=0}^{2}=0 \\
& C:=0,85 \cdot f^{\prime} \cdot b \cdot \beta_{1} \cdot c=4.581 \cdot 10^{k} \\
& R_{v_{0}}:=f_{v_{0}} \cdot A_{v_{0,0}^{\prime}}=-6.293 \cdot 10^{5} \quad R_{v_{1}}:=f_{v_{1}} \cdot A_{s l_{0,7}}=-2.7^{2} 26 \cdot 10^{\circ} \\
& R_{s_{z}}:=f_{s_{2}} \cdot A_{s_{0,2}}=-1.437 \cdot 10^{\circ} \quad R_{s_{3}}:=f_{s_{3}} \cdot A_{s t_{0,3}}=0
\end{aligned}
$$




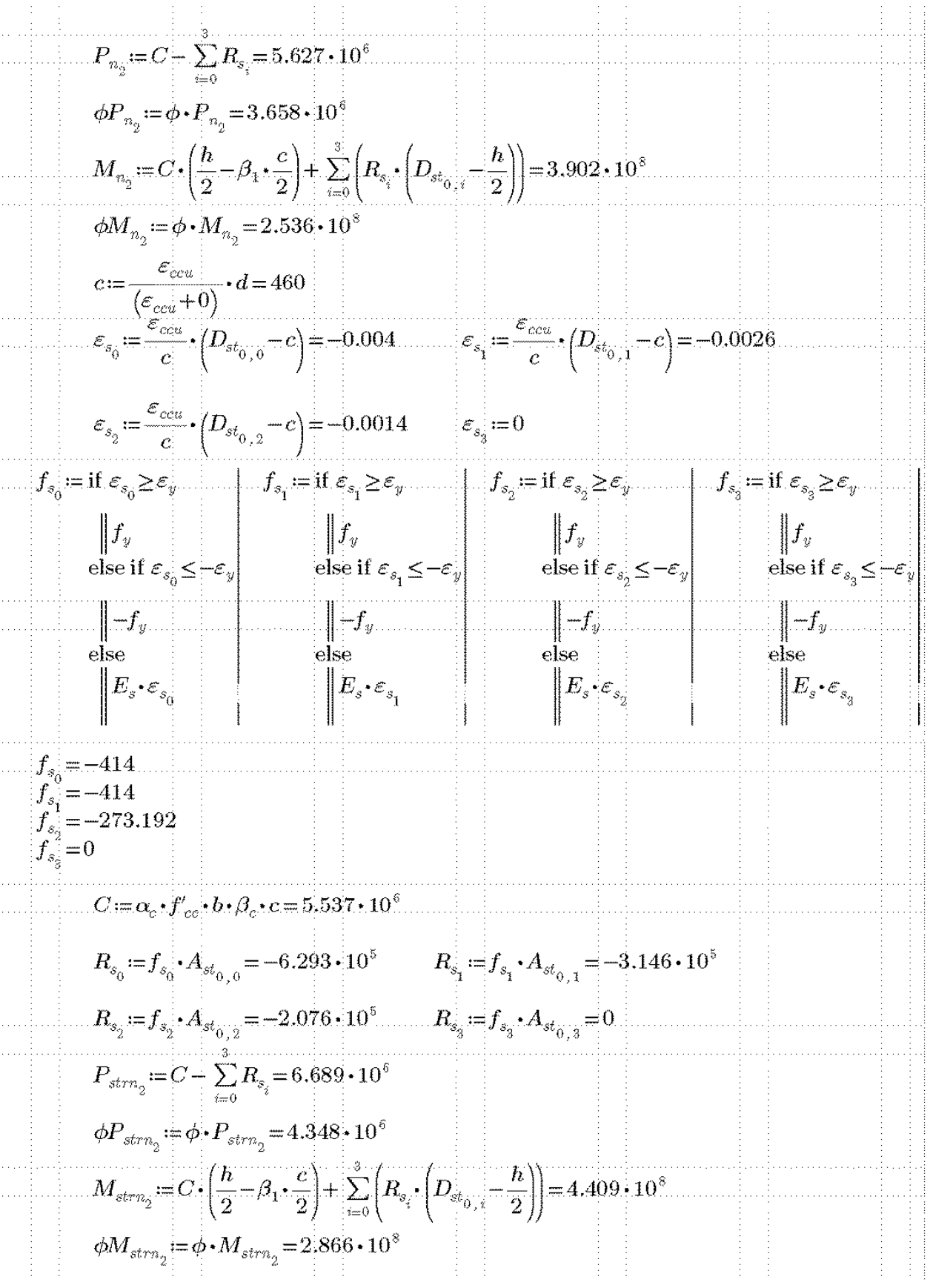


Point Csteel yeilds and concrete crushes simultaneously

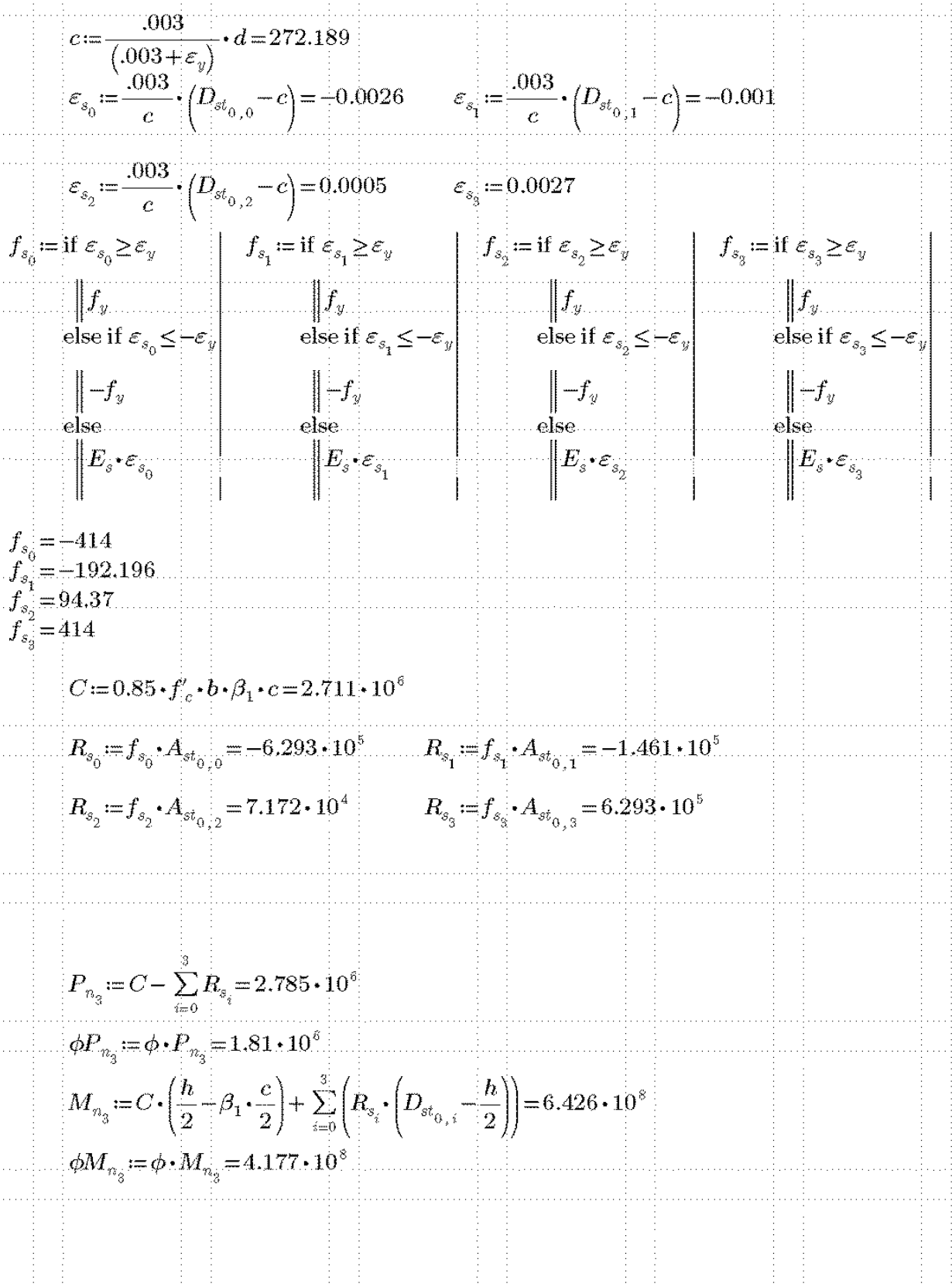




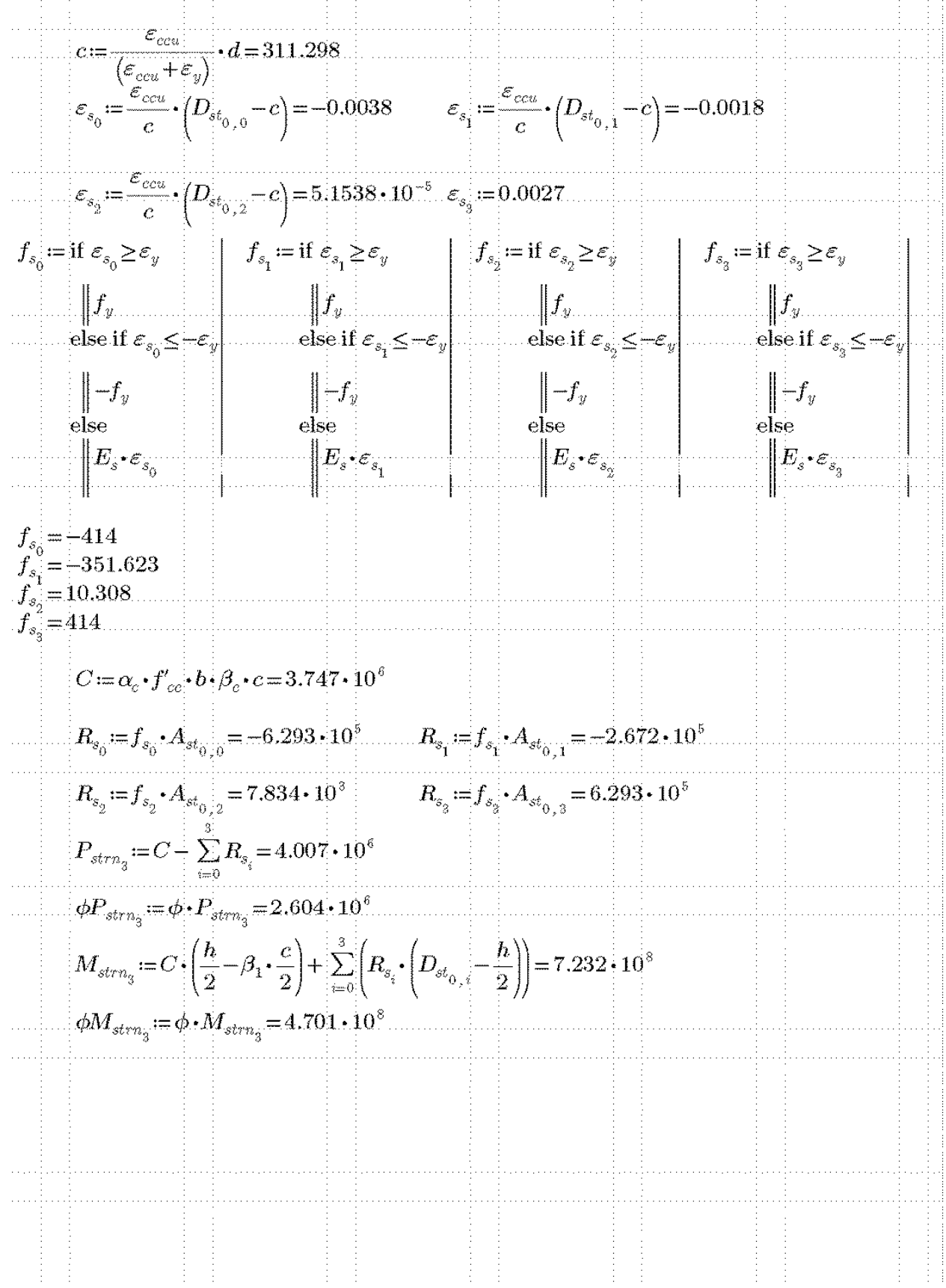




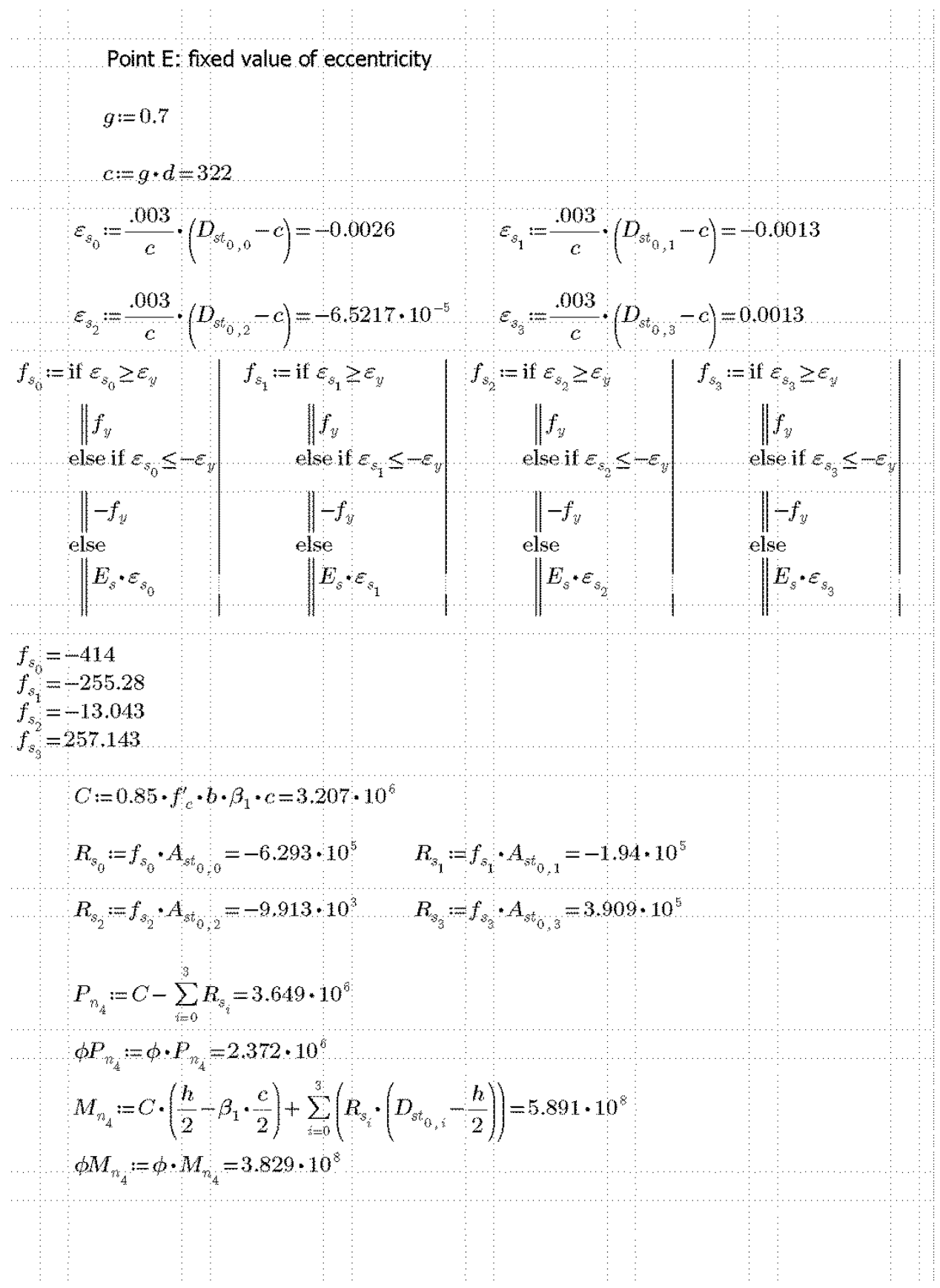




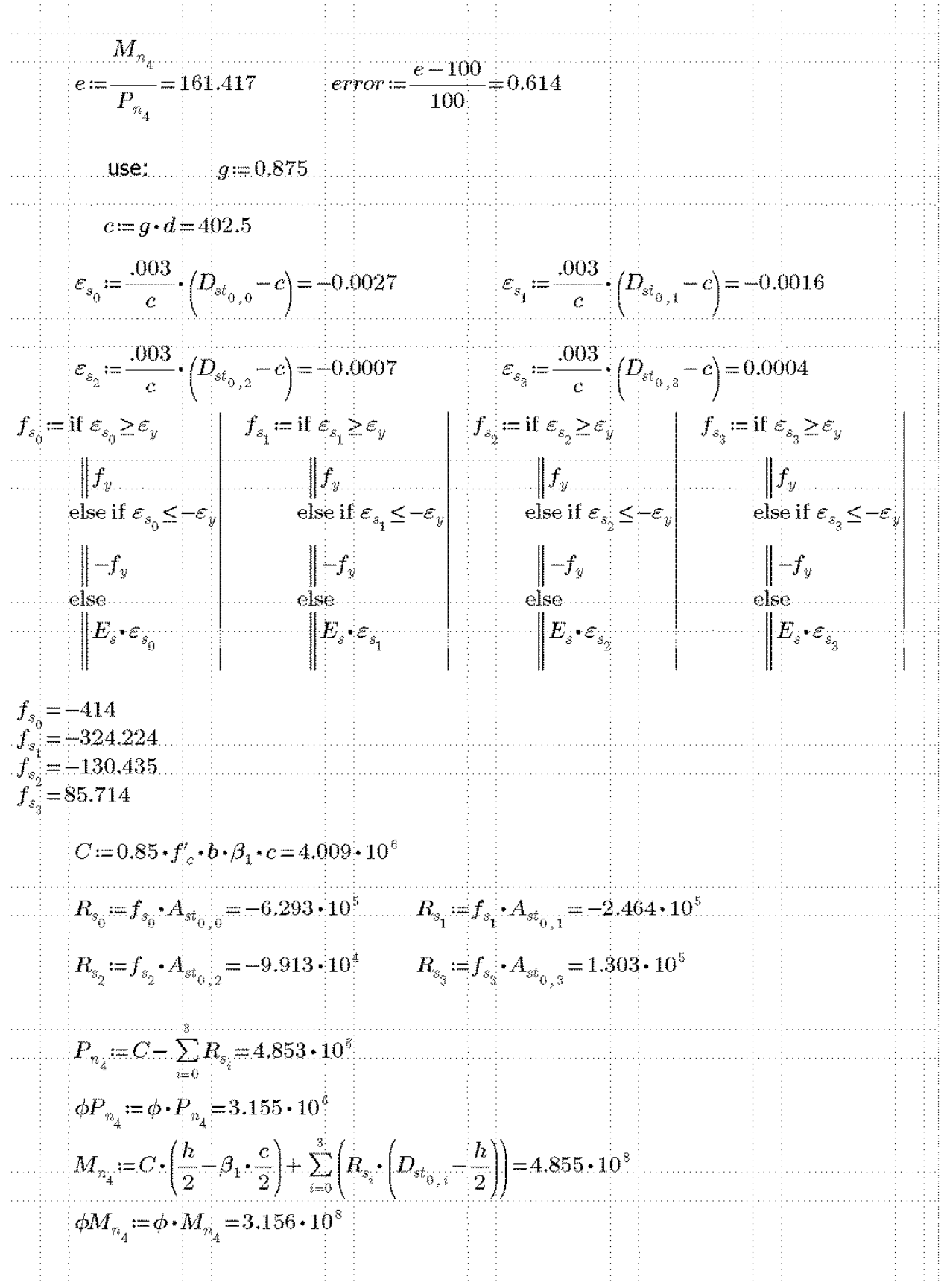




$$
\begin{aligned}
& e=\frac{M_{n_{4}}}{P_{n_{4}}}=100.04 \quad \text { error }:=\frac{e-100}{100}=3.995 \cdot 10^{-4} \\
& e:=0.868 \cdot d=399.28 \\
& \varepsilon_{s_{0}}:=\frac{\varepsilon_{c c u}}{c} \cdot\left(D_{s \hat{s}_{0}, 0}-\varepsilon\right)=-0.0039 \quad \varepsilon_{s_{1}}:=\frac{\varepsilon_{c c u}}{c} \cdot\left(D_{s t_{0}, 1}-c\right)=-0.0023 \\
& \varepsilon_{\mathrm{s}_{i}}:=\frac{\varepsilon_{\mathrm{ccu}}}{c} \cdot\left(D_{\mathrm{s} b_{0}, 2}-c\right)=-0.0009 \quad \ldots \varepsilon_{\mathrm{s}_{3}}=\frac{\varepsilon_{c c u}}{c} \cdot\left(D_{s t_{0,3}}-c\right)=0.0007
\end{aligned}
$$

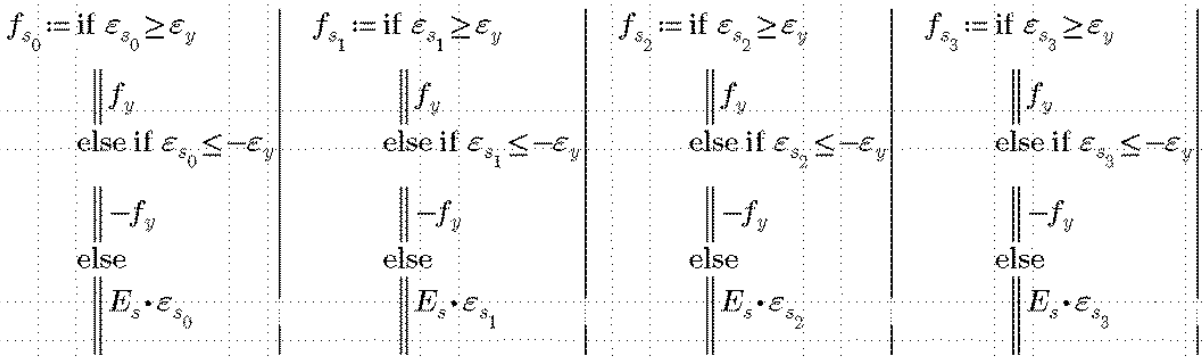

$$
\begin{aligned}
& f_{s}=-414 \\
& f_{s}=-414 \\
& f_{s}=-182.939 \\
& f_{3}^{2}=131.799 \\
& C:=\alpha_{c} \cdot f_{c c}^{\prime \prime} \cdot b \cdot \beta_{c} \cdot c=4.806 \cdot 10^{i z} \\
& R_{s_{0}}:=f_{s_{i}} \cdot A_{s_{0}, 0}=-6.293 \cdot 10^{5} \quad R_{s_{1}}=f_{s_{i}} \cdot A_{s_{0,1}}=-3.146 \cdot 10^{1}
\end{aligned}
$$

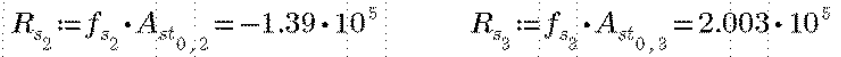

$$
\begin{aligned}
& P_{s t r R_{1}}:=C-\sum_{i=0}^{3} R_{s_{s}}=5.689 \cdot 10^{6}
\end{aligned}
$$

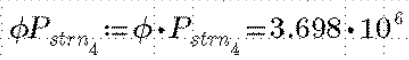

$$
\begin{aligned}
& M_{s t r m_{\alpha}}:=C \cdot\left(\frac{h}{2}-\beta_{1} \cdot \frac{c}{2}\right)+\sum_{i=0}^{3}\left(R_{s_{i}} \cdot\left(D_{s \varepsilon_{0, i}}-\frac{h}{2}\right)\right)=5.716 \cdot 10^{8} \\
& \phi M_{\text {strm }_{4}}:=\phi \cdot M_{\text {stmo }_{4}}=3.715 \cdot 10^{8}
\end{aligned}
$$

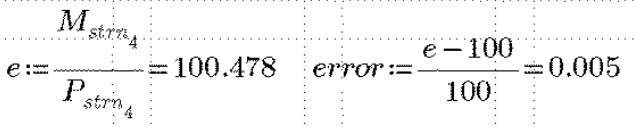




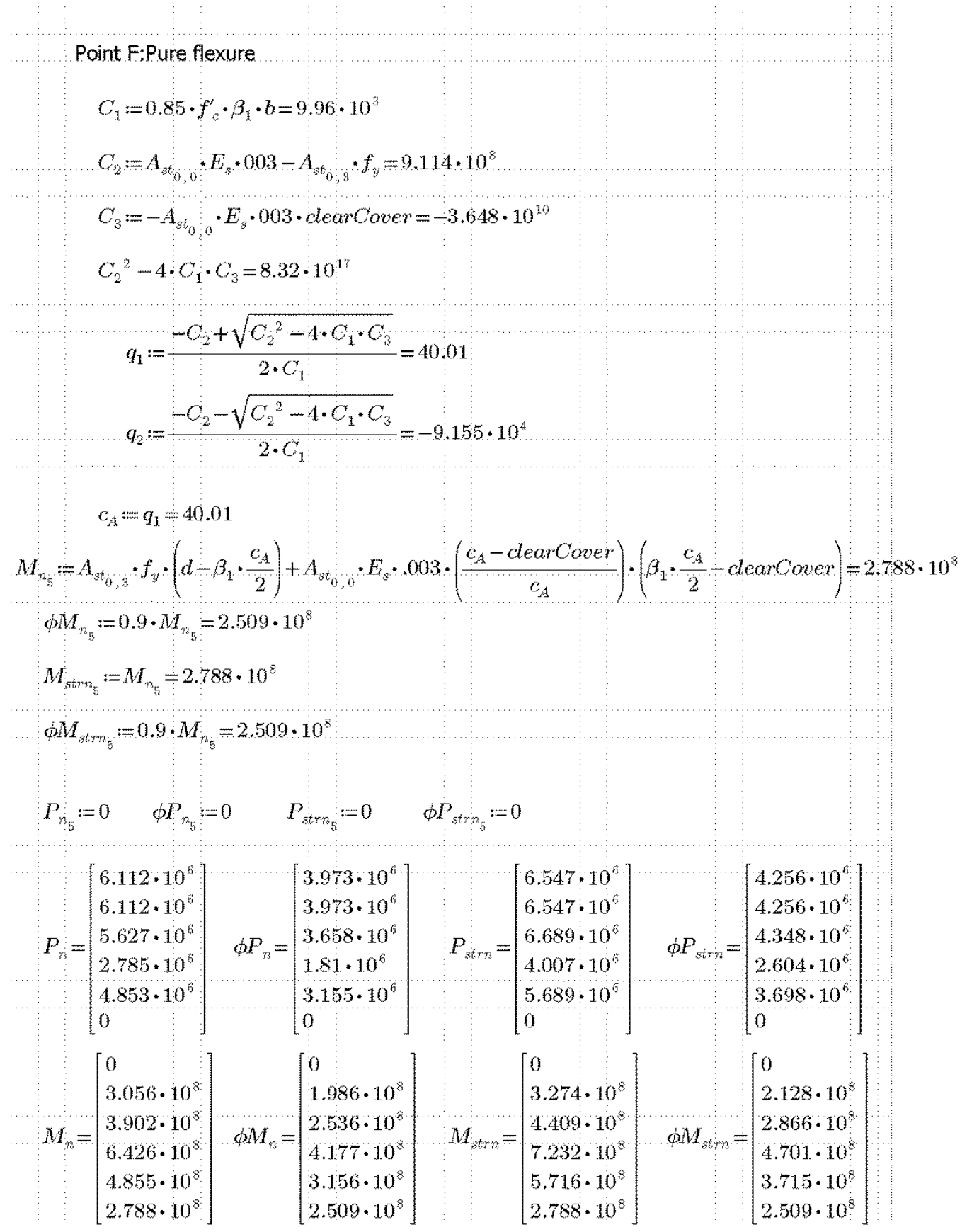




\section{Example L}

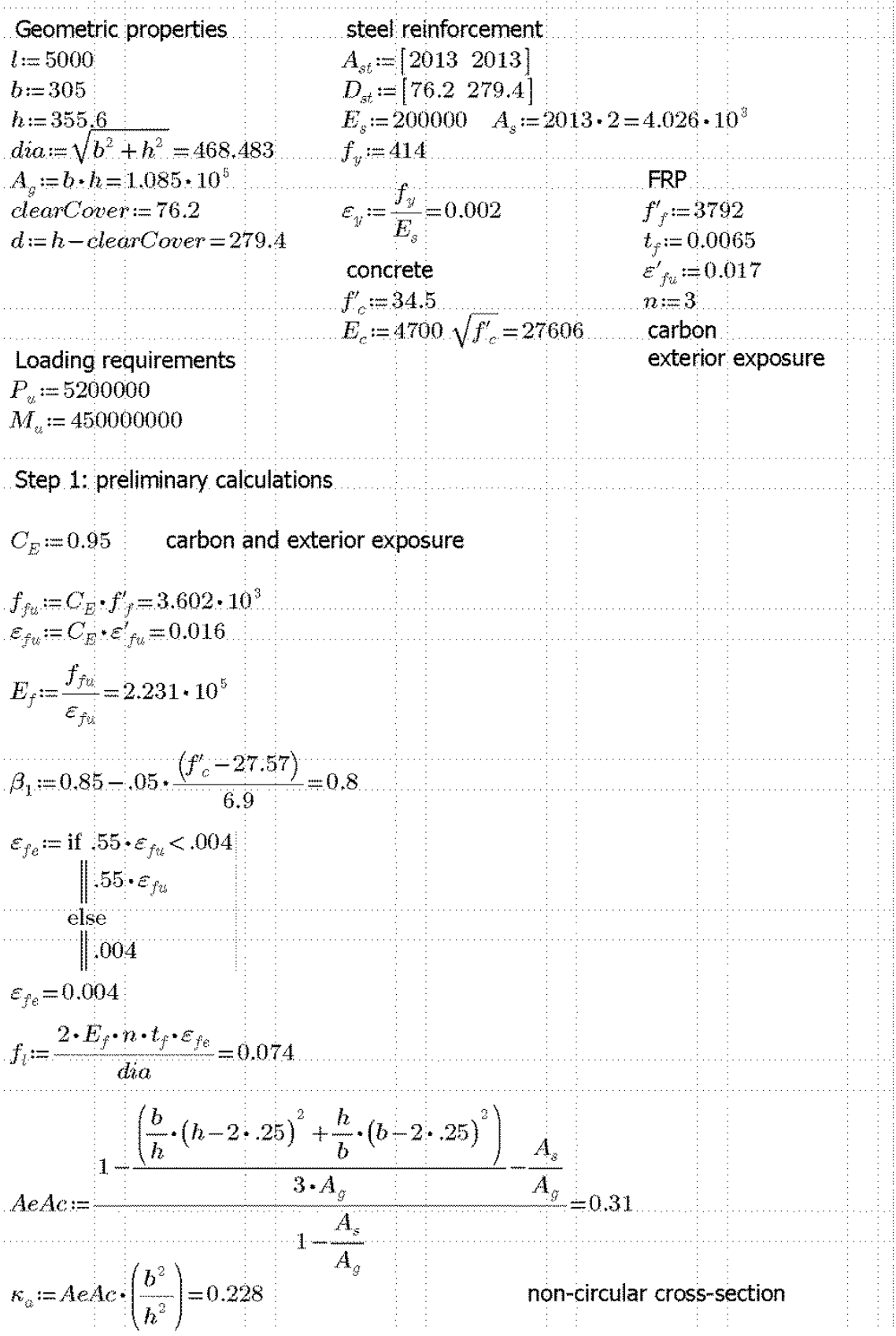




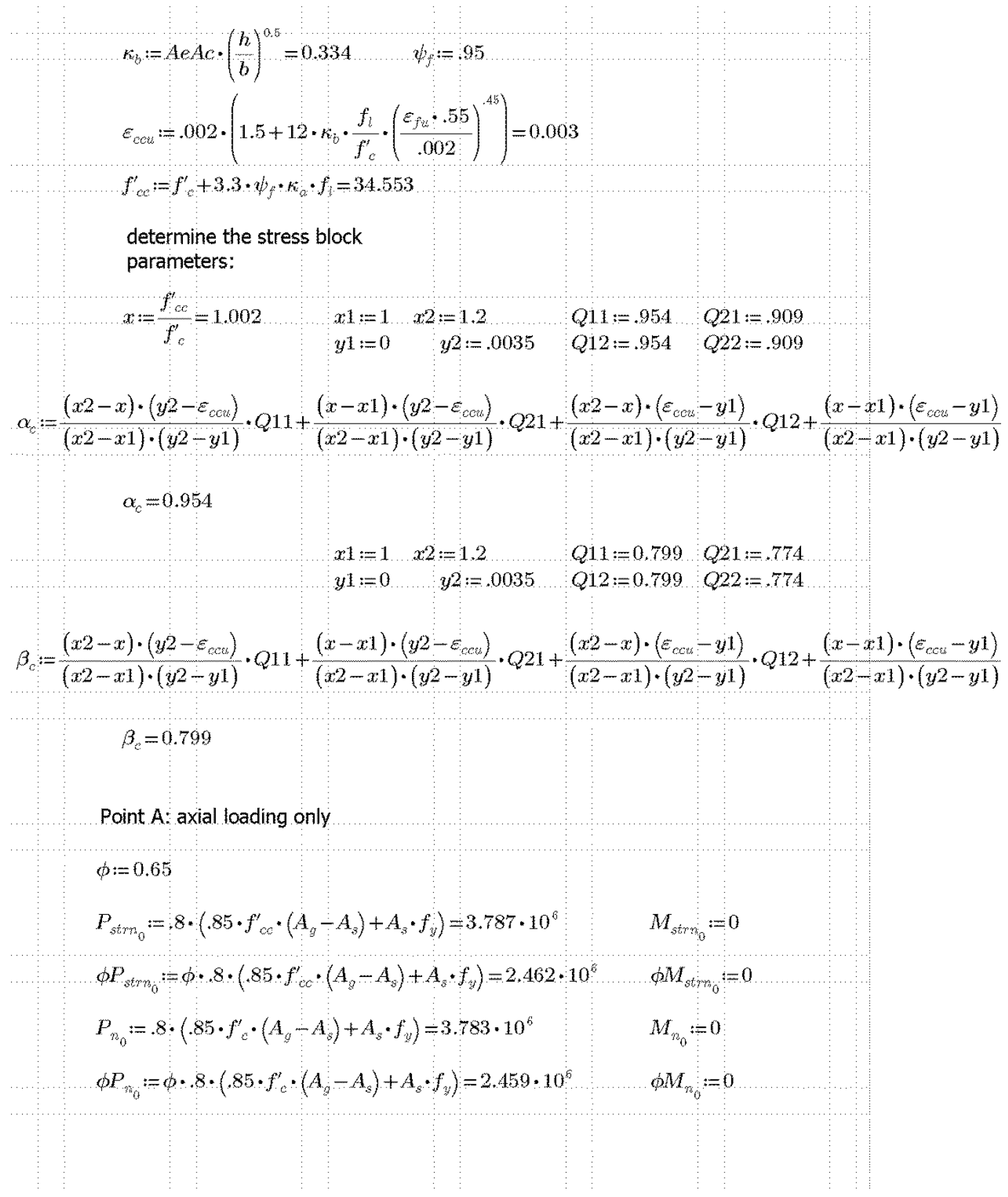


Point D: axial loading with minimum eccentricity

$$
\begin{aligned}
& P_{s t m_{1}}:=P_{s t m_{0}}=3.787 \cdot 10^{6} \quad \quad M_{s t r m_{1}}:=P_{s t m_{1}} \cdot 1 \cdot h=1.347 \cdot 10^{8}
\end{aligned}
$$

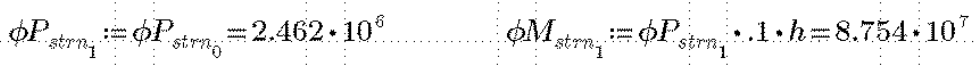

$$
\begin{aligned}
& P_{r_{1}}:=P_{s_{0}}=3.783 \cdot 10^{6} \quad \quad M_{r_{1}}:=P_{n_{1}} \cdot 1 \cdot h=1.345 \cdot 10^{8}
\end{aligned}
$$

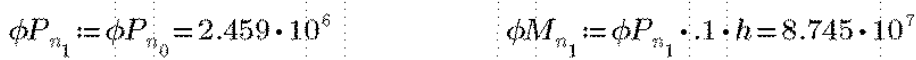

Point B. concrete strain $=.003$ and farthest steel yeiled $c:=\frac{.003}{(.003+0)} \cdot d=279.4$ $\varepsilon_{s_{j}}:=\frac{.003}{c} \cdot\left(D_{s t_{0,0}}-c\right)=-0.0022 \quad \varepsilon_{\mathrm{s}}=0$

$f_{B_{0}}:-$ if $\varepsilon_{B_{0}} \geq \varepsilon_{3}$

$f_{y}$ else if $\varepsilon_{s_{0}} \leq-\varepsilon_{\text {夘 }}$ $\|-f_{z}^{*}$ else $\| E_{s} * E_{s_{0}}$

$f_{s_{i}}=-414$ $f_{3}=0$

$C:=0.85 \cdot f^{\prime} \cdot b \cdot \beta_{1} \cdot c=1.999 \cdot 10^{6}$

$$
R_{s_{0}}:=f_{s_{0}} \cdot A_{s_{0,0}}=-8.334 \cdot 10^{3} \quad R_{s_{1}}:=f_{s_{1}} \cdot A_{s t_{0,1}}=0
$$

dse if $\varepsilon_{s_{1}} \leq-\varepsilon_{y}$

$\|-f$ else $\| E_{s} \cdot \sigma_{3}$

$$
\begin{array}{cccc}
\vdots & \vdots & \vdots & \vdots \\
\vdots & \vdots & \vdots & \vdots \\
\vdots & \vdots & \vdots & \vdots \\
\vdots & \vdots & \vdots & \\
\hdashline
\end{array}
$$




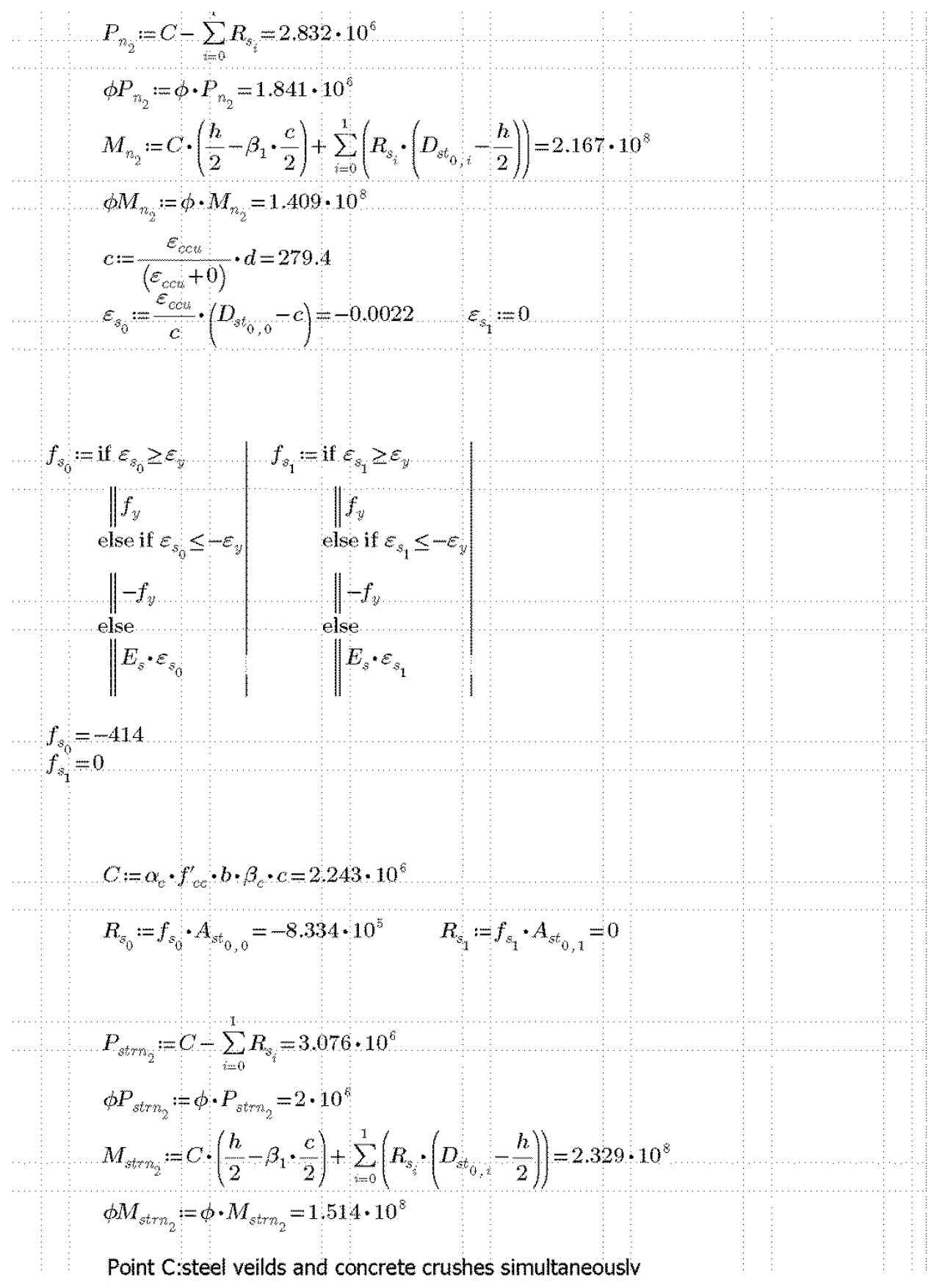




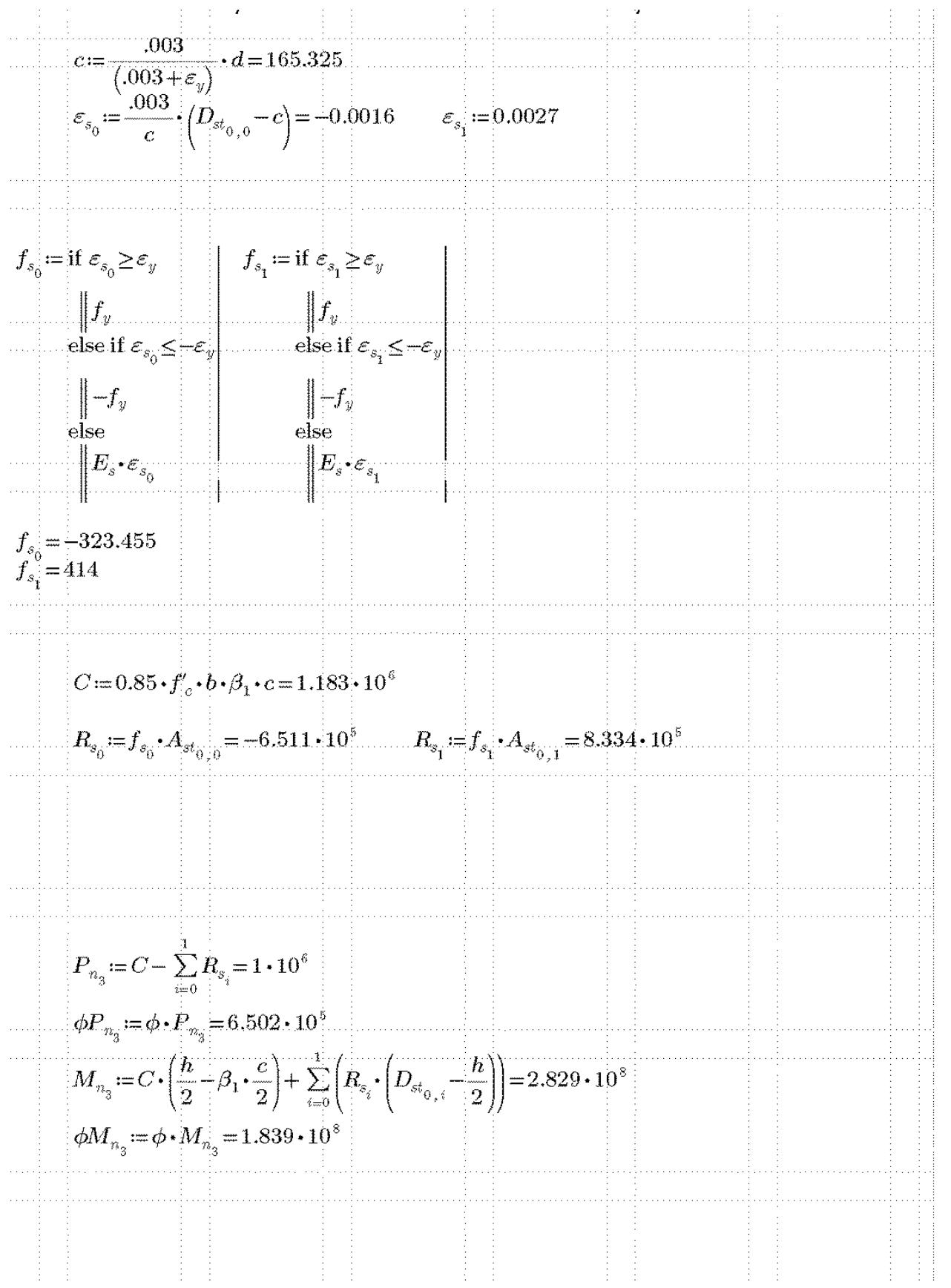




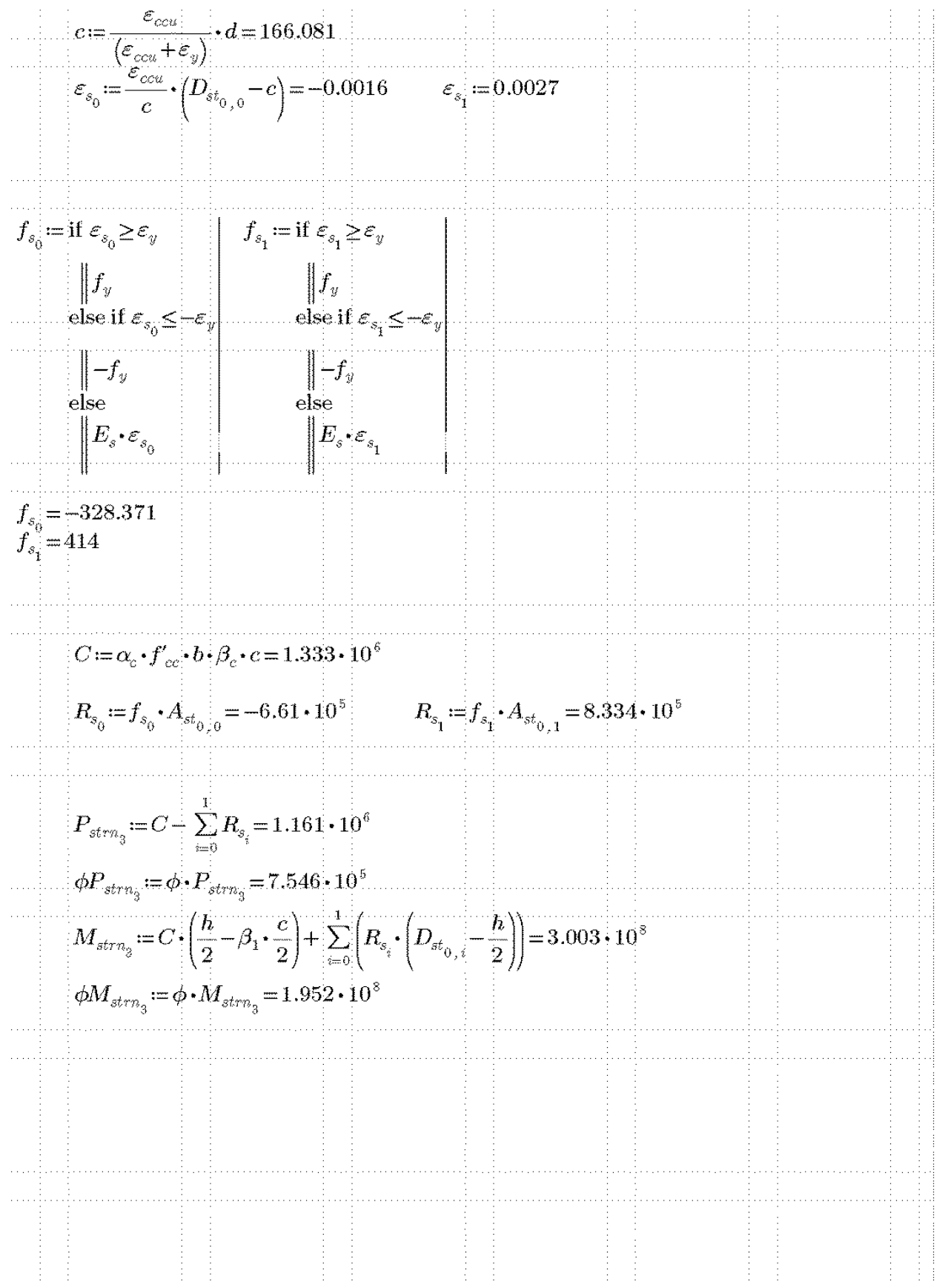




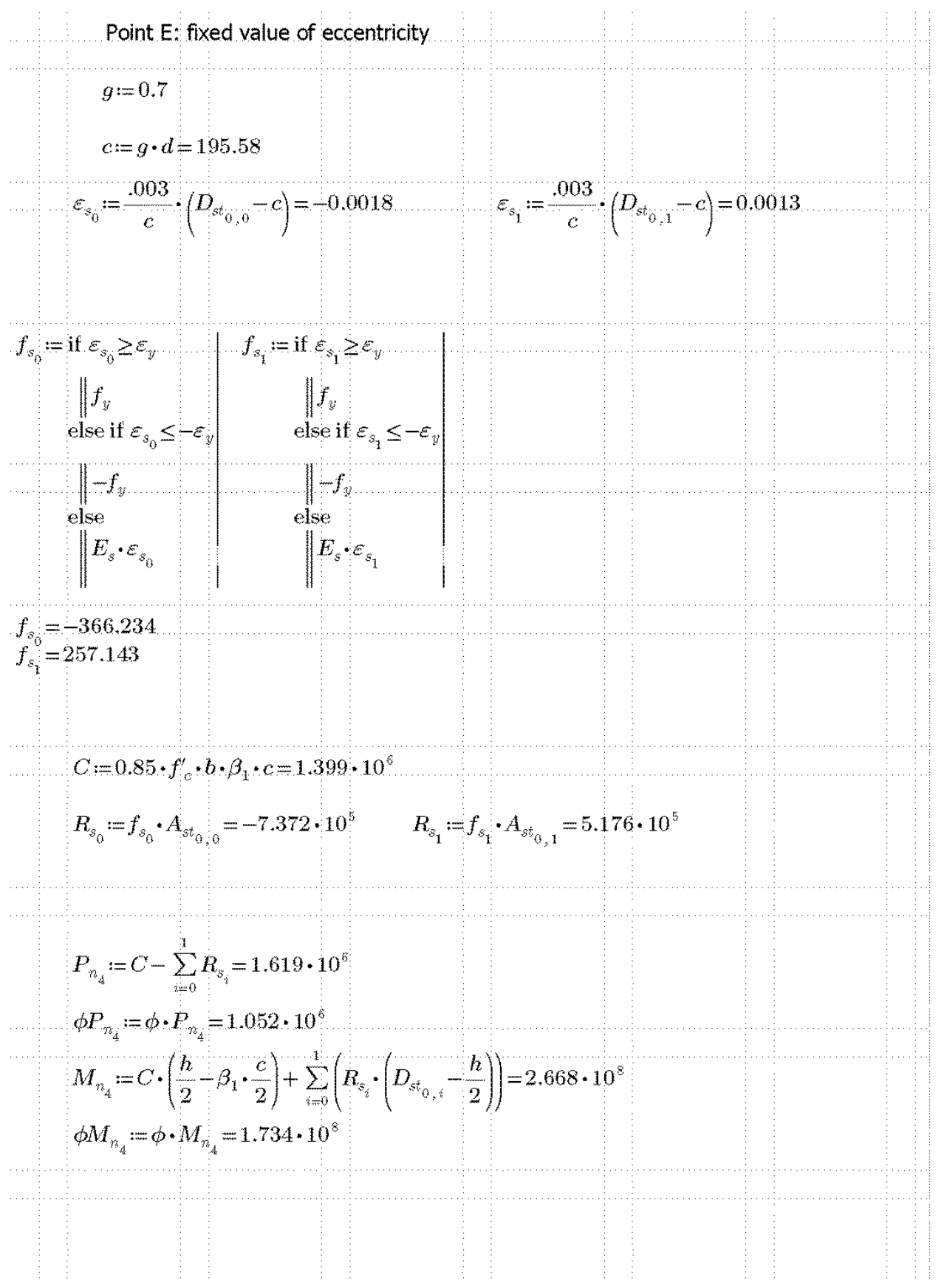









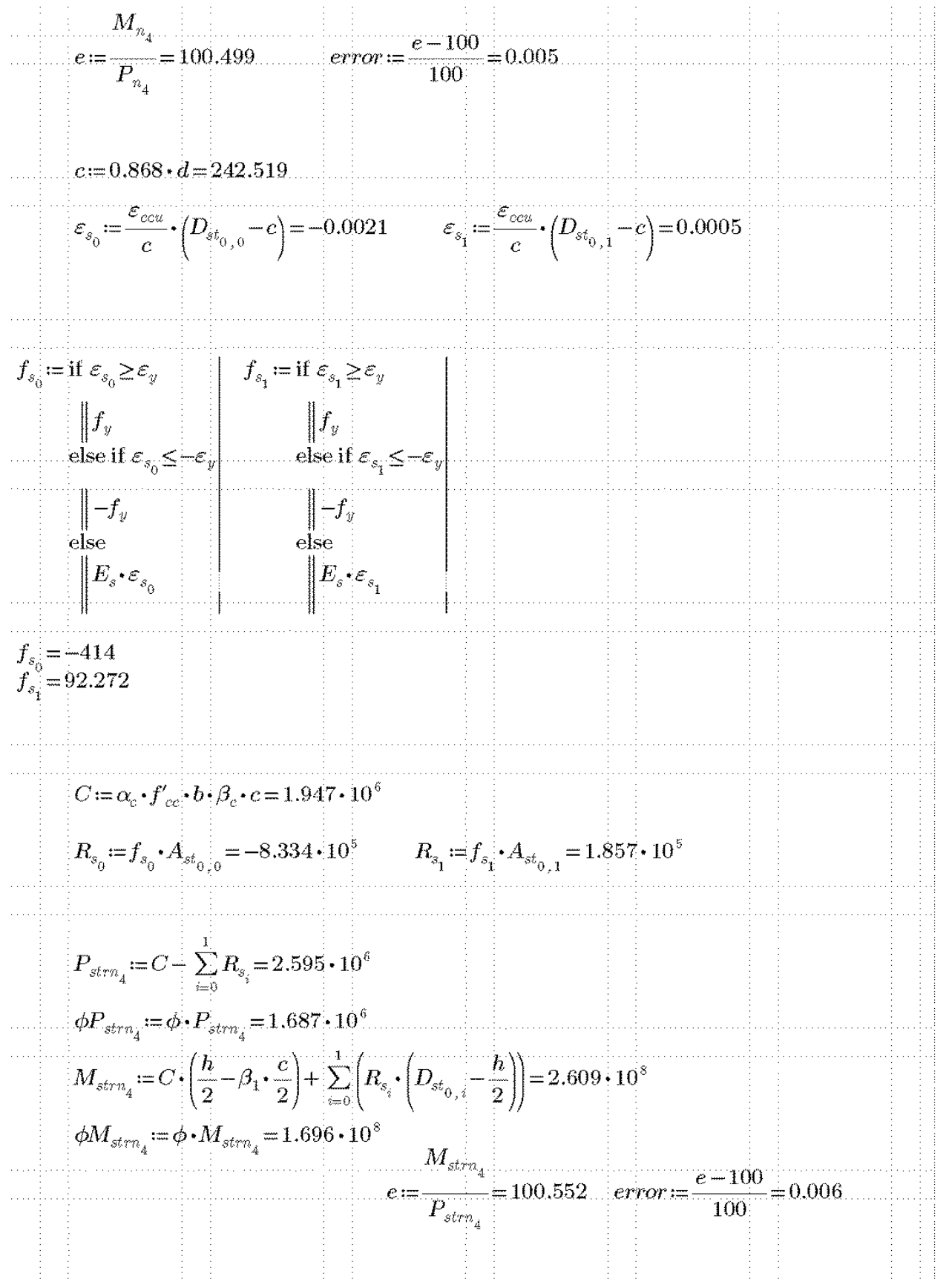




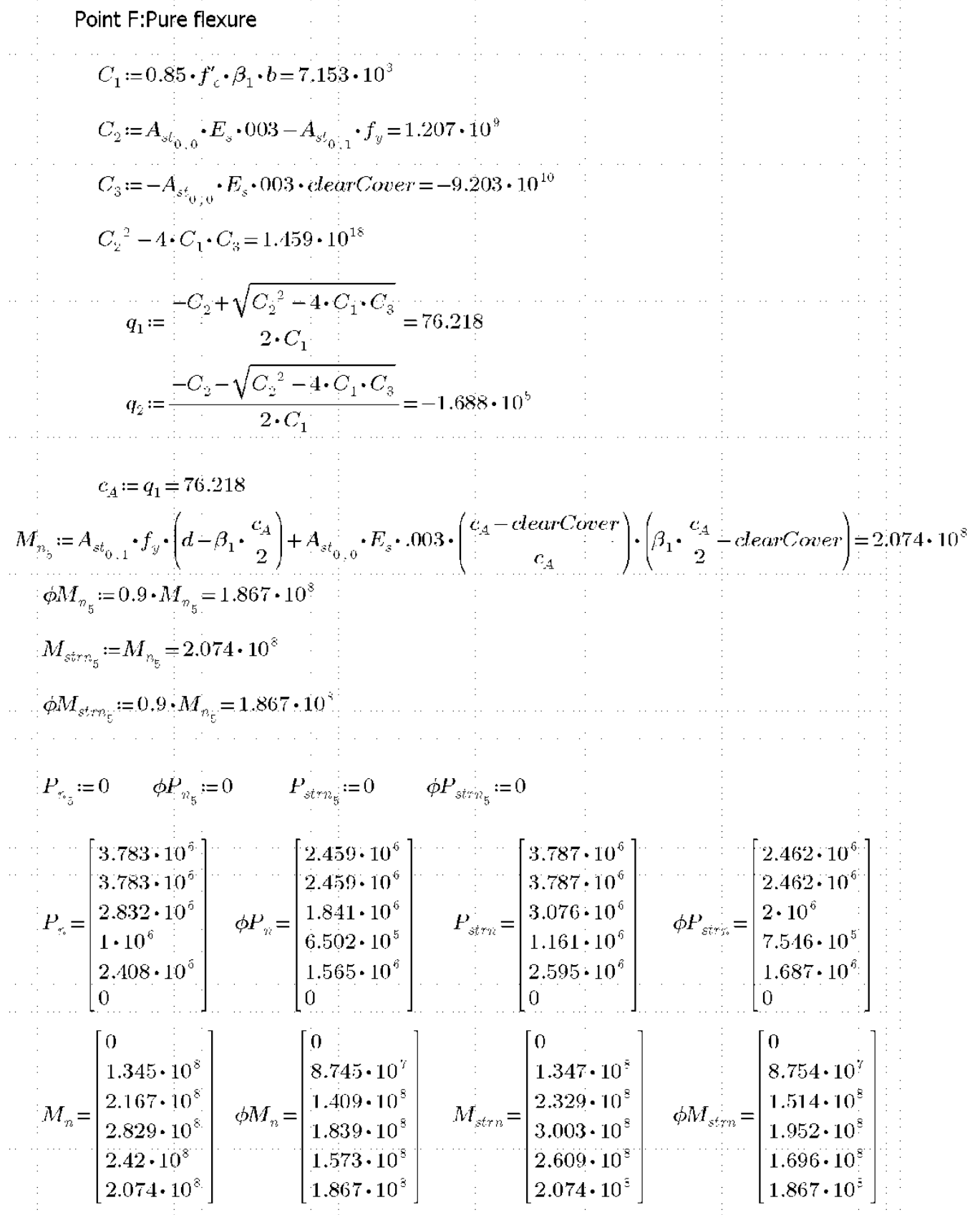

